Supporting Information

\title{
Broadening the chemical space of mussel-inspired polymerization: The roll-out of a TCC-polymer platform with thiol-catechol connectivities.
}

\author{
Jana M. Krüger ${ }^{a}$, Ching-Yi Choi ${ }^{a}$, Francisco Lossada ${ }^{a}$, Peng Wang ${ }^{b}$, Oliver Löschke ${ }^{b}$, \\ Dietmar Auhl ${ }^{b}$, Hans G. Börner ${ }^{a, *}$ \\ ${ }^{a}$ Humboldt-Universität zu Berlin, Department of Chemistry, Laboratory for Organic Synthesis \\ of Functional Systems, Brook-Taylor Straße 2, 12489 Berlin, Germany \\ b Technische Universität Berlin, Department of Polymer Materials and Technologies, Ernst \\ Reuter-Platz 1, 10587 Berlin, Germany
}

Journal: Macromolecules

Prof. Dr. Hans Börner

Humboldt-Universität zu Berlin

Laboratory for Organic Synthesis of Functional Systems

Brook-Taylor-Str. 2, 12489 Berlin

Germany

E-Mail: h.boerner@hu-berlin.de

Web: http://www.boernerlab.de

ORCID Publication ID: 0000-0001-9333-9780

Phone: +49 (0)30-2093 7348

Fax: $+49(0) 302093-7500$ 


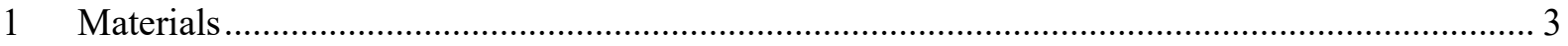

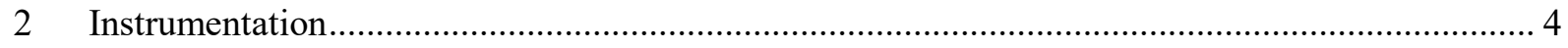

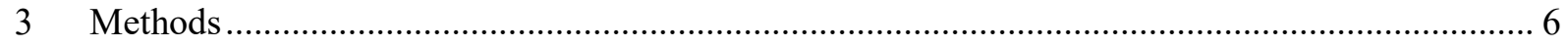

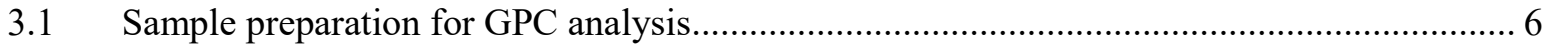

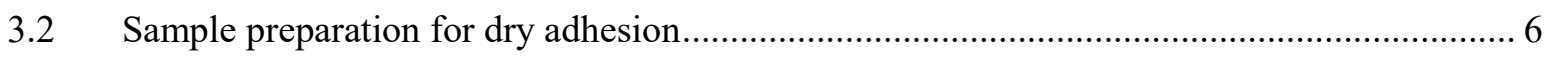

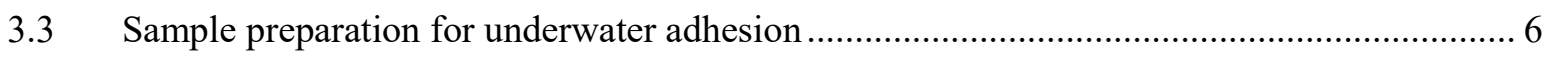

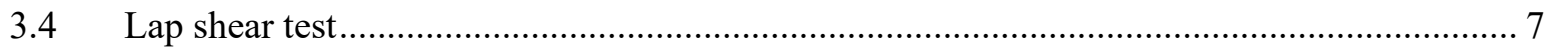

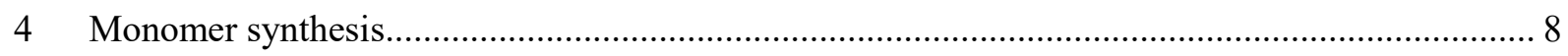

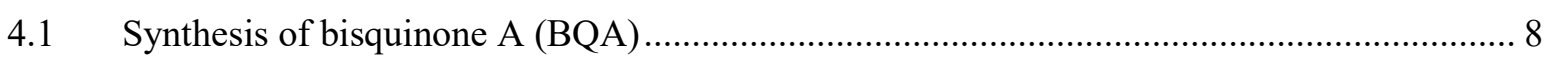

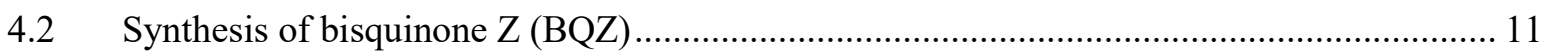

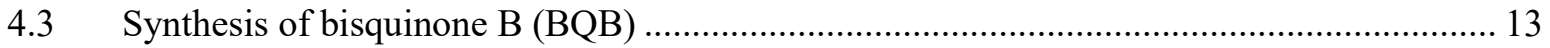

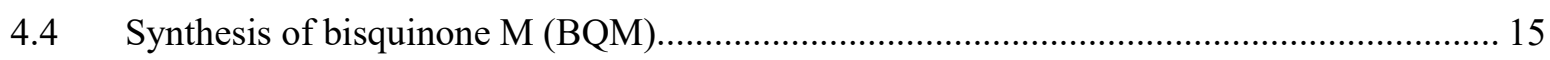

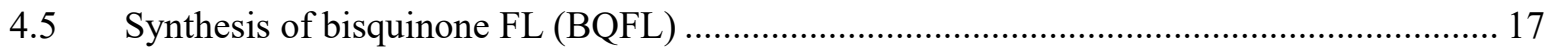

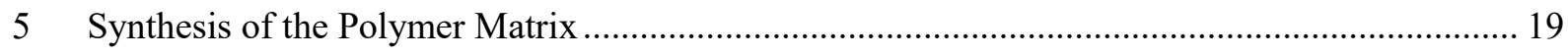

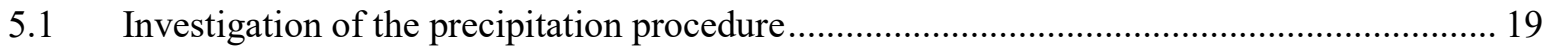

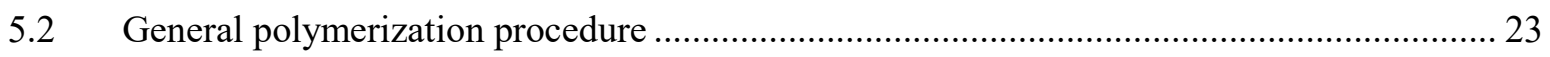

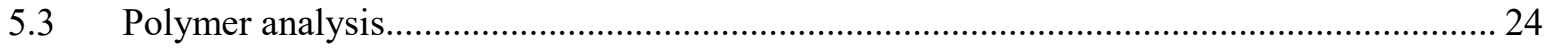

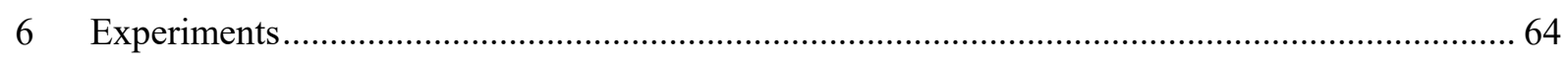

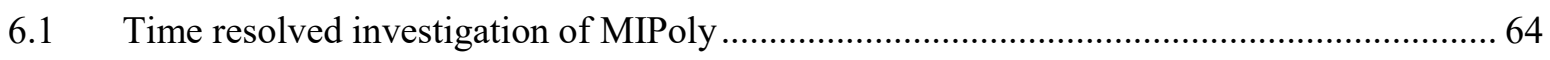

6.2 Calculation of the proportion of low molecular weight products............................................ 66

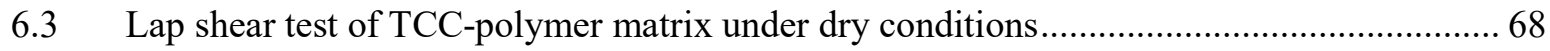

6.4 Exemplary pictures and SEM analysis of the test specimen surfaces after adhesive testing of

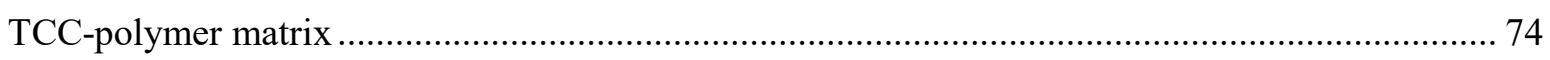

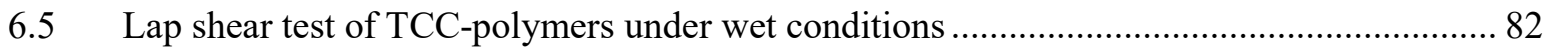

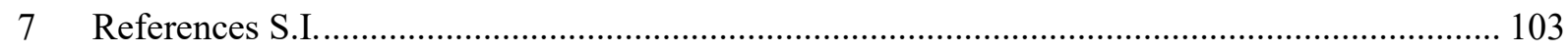




\section{Materials}

Oxone $^{\circledR}$ (monopersulfate compound), 2-iodobenzoic acid, 2,6-di-tertbutyl-4-methylphenol (BHT, <99\%), 2,2'-(diethylenedioxy)-diethanethiol (95\%), dithiothreitol (99\%), dithranol, bisphenol M, sodium tetrafluoro acetate and tributyl phosphine were purchased from Sigma Aldrich $^{\circledR}$ Chemie GmbH (Seelze, Germany) and bisphenol A, bisphenol Z, 1,4bismercaptobenzene (98\%), 2,3-dimercapto-1-propanol (95\%), bisphenol B, bisphenol FL, 1,4butanedithiol (95\%), 1,6-hexanedithiol (96\%) from TCI (Tokyo, Japan). Deuterated dimethyl sulfoxide (DMSO- $\mathrm{d}_{6}$ ) was obtained from deuteron (Kastellaun, Germany). Glycol dimercaptoacetate and glycol dimercaptopropionate (Bruno Bock Chemische Fabrik GmbH \& Co. KG (Marschacht, Germany)). N-Methyl-2-pyrrolidon (NMP) was purchased from Iris Biotech GmbH (Marktredwitz, Germany), tetrahydrofuran (LC-MS grade) and acetonitrile (LC-MS grade) were obtained from $V W R^{\circledR}$ chemicals (Dresden, Germany) and formic acid (LCMS grade) from Merck KGaA (Darmstadt, Germany).

Iodoxybenzoic acid (IBX) was synthesized according to the procedure described by Frigerio et al. ${ }^{1}$ CAUTION! IBX was found to be explosive under heating $>200{ }^{\circ} \mathrm{C}$ or impact! ${ }^{2}$

Milli-Q water was produced using SG LaboStar ${ }^{\circledR}$ TM 1-UV system from $S G$ water (Hamburg, Germany). As ion exchanger, Evoqua Water Technologies Polisher HP2 module was inserted. Electric conductibility of Milli-Q water was $0,055 \mu \mathrm{S} \mathrm{cm}^{-1}$. 


\section{Instrumentation}

Ultra-performance liquid chromatography with electron spray ionization mass spectrometry (UPLC-ESI-MS) was carried out on an ACUIDITY-UPLC ${ }^{\circledR}$ H-Class CM Core System of Waters GmbH (Eschborn, Germany). Detection was done utilizing an ACUIDITYUPLC ${ }^{\circledR}$ photo diode array (PDA)-detector (wavelength range 190-500 nm) and an ACUIDITYUPLC $^{\circledR}$ QDa mass detector with ESI-ionization. For analysis Waters software Empower ${ }^{\mathrm{TM}_{3}}$ was used. Separation was conducted with ACUIDITY-UPLC ${ }^{\circledR}$ BEH C18 VanGuard $^{\mathrm{TM}}$ precolumn $\left(110 \AA, 1.7 \mu \mathrm{m}, 5 \times 21 \mathrm{~mm}\right.$ ID) and an ACUIDITY-UPLC ${ }^{\circledR}$ BEH C18-column (110 ̊, $1.7 \mu \mathrm{m}, 5 \times 21 \mathrm{~mm}$ ID) from Waters. As mobile phase, mixtures of solvent A (Milli-Q water with $0.1 \% \mathrm{FA}, \mathrm{v} / \mathrm{v}$ ) and solvent $\mathrm{B}$ (acetonitrile with $0.1 \% \mathrm{FA}, \mathrm{v} / \mathrm{v}$ ) were used with 0.5 $\mathrm{mL} \min ^{-1}$ flow rates.

Gel permeation chromatography (GPC) was carried out on an Eco-SEC-System with UV and RI-detection (HLC-8320 GPC) from Tosoh (Griesheim, Germany). As solvent tetrahydrofuran (THF, HiPerSolv CHROMANORM ${ }^{\circledR}$ for HPLC) from $V W R^{\circledR}$ Chemicals (Dresden, Germany) was used and SDV columns (1000 $5 \mu \mathrm{m}, 100000 \AA 5 \mu \mathrm{m}$ and $1000000 \AA 5 \mu \mathrm{m}$ ) from PSS (Mainz, Germany) were applied.

MALDI-TOF-MS/MS measurements were carried out on a MALDI-TOF/TOF 5800 system (AB Sciex, USA). As matrix a mixture of $1: 1$ dithranol $\left(20 \mathrm{mg} \mathrm{mL}^{-1}\right)$ and NaTFA $\left(5 \mathrm{mg} \mathrm{mL}^{-1}\right)$ in THF was used.

Nuclear magnetic resonance spectroscopy (NMR) was performed on an Avance II 500 spectrometer (Bruker BioSpin GmbH, Rheinstetten, Germany).

Fourier transform infrared spectroscopy (FT-IR) was carried out on a Brucker Vertex 70v FT-IR spectrometer (Bruker Optik GmbH, Ettlingen, Germany).

Thermo gravimetric analysis (TGA) was carried out on a TGA/SDTA851e system (Mettler Toledo, USA). 
Differential scanning calorimetry (DSC) measurements were performed on DSC822e Differential Scanning Calorimeter (Mettler Toledo, USA). Heating rate $10 \mathrm{~K} / \mathrm{min}$.

Shear tests have been carried out with a Texture Analyzer Ta.XT.plus100C (Stable Micro Systems, Godalming, United Kingdom) with a $50 \mathrm{~kg}$ force cell.

For shear test sample preparation, a manual hydraulic press with a west $6100+$ temperature controller from Specac Ltd Science and Innovation Centre (Orpington, United Kingdom) was utilized.

Scanning Electron Microscopy (SEM) was carried out using a Tabletop Microsope TM1000 from Hitachi (Chiyoda, Japan). 


\section{Methods}

\subsection{Sample preparation for GPC analysis}

The dried polymer solid was dissolved in THF and filtered through $0.2 \mu \mathrm{m}$ PTFE filter. $1.5 \mathrm{~mL}$ of the filtrated solution were transferred into glass vials and mixed with $7.5 \mu \mathrm{L}$ of BHT-standard $(50 \mathrm{mg} / \mathrm{mL}$ solution in THF).

\subsection{Sample preparation for dry adhesion}

Aluminum $(5005 \mathrm{~A}, 80 \mathrm{~mm} \times 20 \mathrm{~mm} \times 1,5 \mathrm{~mm})$ and poly(propylene) $(\mathrm{PP})$ plates (Simona DWST, $80 \mathrm{~mm} \times 25 \mathrm{~mm} \times 3 \mathrm{~mm}$ ) with a hole at one narrow side of the plates were cleaned with acetone and pre-treated with UV/Ozone for $30 \mathrm{~min}$. Subsequently, a defined area of the test specimen $(20 \mathrm{~mm} \times 7 \mathrm{~mm}$ for aluminum and $25 \mathrm{~mm} \times 10 \mathrm{~mm}$ for PP) was coated with a solution of the corresponding polymer in acetone $(167 \mathrm{mg} / \mathrm{mL}, 20 \mu \mathrm{L}$ on aluminum and $30 \mu \mathrm{L}$ on PP). The solvent was evaporated at room temperature and the coated surfaces of two test specimen were combined. Curing was carried out in a "hot-melt"-like process in which the adhesive was cured for $15 \mathrm{~min}$ at $120^{\circ} \mathrm{C}$ in a hot press, while applying $1000 \mathrm{~kg}$ weight for each test specimen. Afterwards the samples were cooled under pressure, removed from the hot press and could equilibrate overnight at r.t..

\subsection{Sample preparation for underwater adhesion}

Underwater adhesion tests were carried out as shown in Figure S 1 with TCC-polymer solutions (60 $\mu \mathrm{L}, 750 \mathrm{mg} / \mathrm{mL}$ in chloroform) for $\mathrm{p}(\mathrm{BQB}-E D E T), \mathrm{p}(\mathrm{BQZ}-E D E T), \mathrm{p}(\mathrm{BQM}-E D E T)$, $\mathrm{p}(\mathrm{BQM}-\mathrm{BMT}), \mathrm{p}(\mathrm{BQM}-\mathrm{GDMP})$ as well as $\mathrm{p}(\mathrm{BQM}-\mathrm{HDT})$. Curing was carried out for 3 days at $50{ }^{\circ} \mathrm{C}$. After that, the water bath was cooled to room temperature and the glued test specimen were directly analyzed in a lap shear arrangement. 
a

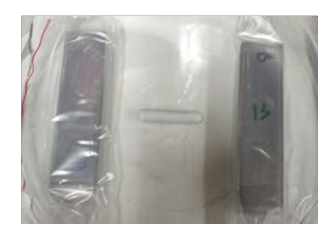

b

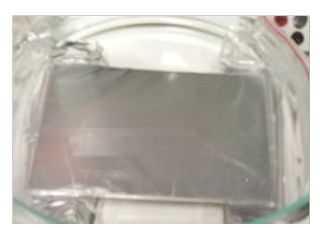

C

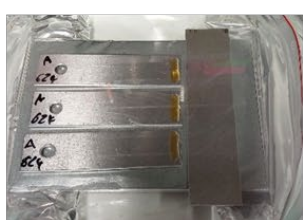

d

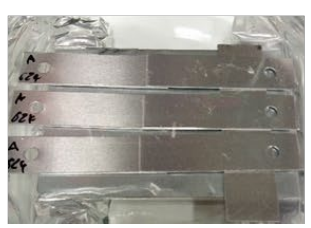

e

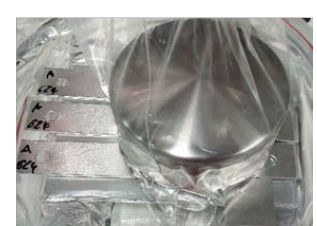

Figure S 1. Construction and implementation of the underwater adhesion tests. Spacers and a magnetic stirring bar were placed in a big crystallizing dish (a) and covered with a metal plate to ensure a flat base (b). The system is filled with $599 \mathrm{mM} \mathrm{NaCl}$-solution. Cleaned and UV/ozone pretreated test specimen were then placed on the metal plate and coated directly under water with a TCC-polymer solution in chloroform (c). A second test specimen is placed on the coated area to create an overlap area of $10 \mathrm{~mm}$ x $20 \mathrm{~mm}$ (d) and the glued samples were hold in place by an applied weight (e).

\subsection{Lap shear test}

Force-distance-curves were measured in a shear arrangement with a velocity of $0.05 \mathrm{~mm} / \mathrm{s}$. The adhesion strength was calculated by normalizing the maximum force of the force-distancecurve to the area of the adhesive overlap of the test specimen. 


\section{Monomer synthesis}

\subsection{Synthesis of bisquinone A (BQA)}<smiles>CC(C)(C1=CC(=O)C(=O)C=C1)C1=CC(=O)C(=O)C=C1</smiles>

Bisphenol A (5.0 g, $20 \mathrm{mmol})$ was dissolved in $200 \mathrm{~mL}$ methanol. The solution was mixed with IBX $(18.5 \mathrm{~g}, 66 \mathrm{mmol})$ and the mixture was stirred for $15 \mathrm{~min}$ at room temperature. Formation of a red solid was observed. Subsequently, the mixture was cooled in an ice bath for $10 \mathrm{~min}$, the red solid was filtrated off and was washed with cold methanol. The red solid was redissolved in chloroform. After filtration, the organic solvent was removed under reduced pressure to obtain a red solid in $5.2 \mathrm{~g}$ yield $(92 \%)$.

NMR spectra are shown in Figure S 2 and Figure S 3.

${ }^{1} \mathrm{H}-\mathrm{NMR}\left(500 \mathrm{MHz}, \mathrm{DMSO}-\mathrm{d}_{6}\right): \delta[\mathrm{ppm}] 7.07$ (dd, J = 10.3, $\left.2.4 \mathrm{~Hz}, 1 \mathrm{H}\right), 6.40-6.34(\mathrm{~m}, 2 \mathrm{H})$, $1.43(\mathrm{~s}, 3 \mathrm{H})$.

${ }^{13} \mathrm{C}-\mathrm{NMR}\left(126 \mathrm{MHz}, \mathrm{DMSO}-\mathrm{d}_{6}\right) \delta[\mathrm{ppm}] 179.72,179.61,155.77,139.90,130.09,126.26,43.71$, 24.16.

FT-IR: 2985 (w), 1686 (m), 1664 (s), 1622 (s), 1564 (m), 1543 (w), 1404 (m), 1285 (m), 1232 (w), $1178(\mathrm{w}), 1147(\mathrm{w}), 1128(\mathrm{w}), 1038(\mathrm{w}), 915(\mathrm{w}), 878(\mathrm{w}), 816(\mathrm{~m}), 682(\mathrm{w}), 647(\mathrm{w}), 616$ (w).

\section{$\underline{\text { UPLC-UV/Vis-QMS: }}$}

$\mathrm{UV} / \mathrm{Vis}(210 \mathrm{~nm}): \mathrm{t}_{\mathrm{R}}=1.915 \mathrm{~min}$ und $2.203 \mathrm{~min}, 99 \%$ purity (solvent A (Milli-Q water with $0.1 \% \mathrm{FA}, \mathrm{v} / \mathrm{v}$ ) and solvent B (acetonitrile with 0.1\% FA), Gradient 10-90\% B).

ESI-QMS: calculated (m/z): 255.06 [M-H] $^{-}, 511.13$ [2M-H]-; found (m/z): $257.07[\mathrm{M}-\mathrm{H}]^{-}$, $515.10[2 \mathrm{M}-\mathrm{H}]^{-}$. The mass differences of $+2 \mathrm{Da}$ and $+4 \mathrm{Da}$ found for all bisquinone monomers was consistent with the literature, describing for various quinones a corona discharge-induced, 
in source reduction in the negative electrospray ionization process. ${ }^{3}$ This in source event was evident for the bisquinone as well, leading to a quinone-catechol structure to occur (calculated $\left.(\mathrm{m} / \mathrm{z}) 257.06[\mathrm{M}-\mathrm{H}]^{-}, 515.13[2 \mathrm{M}-\mathrm{H}]^{-}\right)$.

The complementing analysis data obtained by IR, ${ }^{1} \mathrm{H}-\mathrm{NMR}$ and ${ }^{13} \mathrm{C}-\mathrm{NMR}$ confirmed the insource artefact by showing clear evidence of bisquinones and exclude mixed quinonecatechol structures as products. IR showed the absence of hydroxy-groups but characteristic vibrational bands of carbonyl-moieties. 1H-NMR confirmed the absence of hydroxy-groups as well and ${ }^{13} \mathrm{C}-\mathrm{NMR}$ showed resonances within chemical shift ranges typical of carbonyl-Catoms between 175 and $225 \mathrm{ppm}$. 


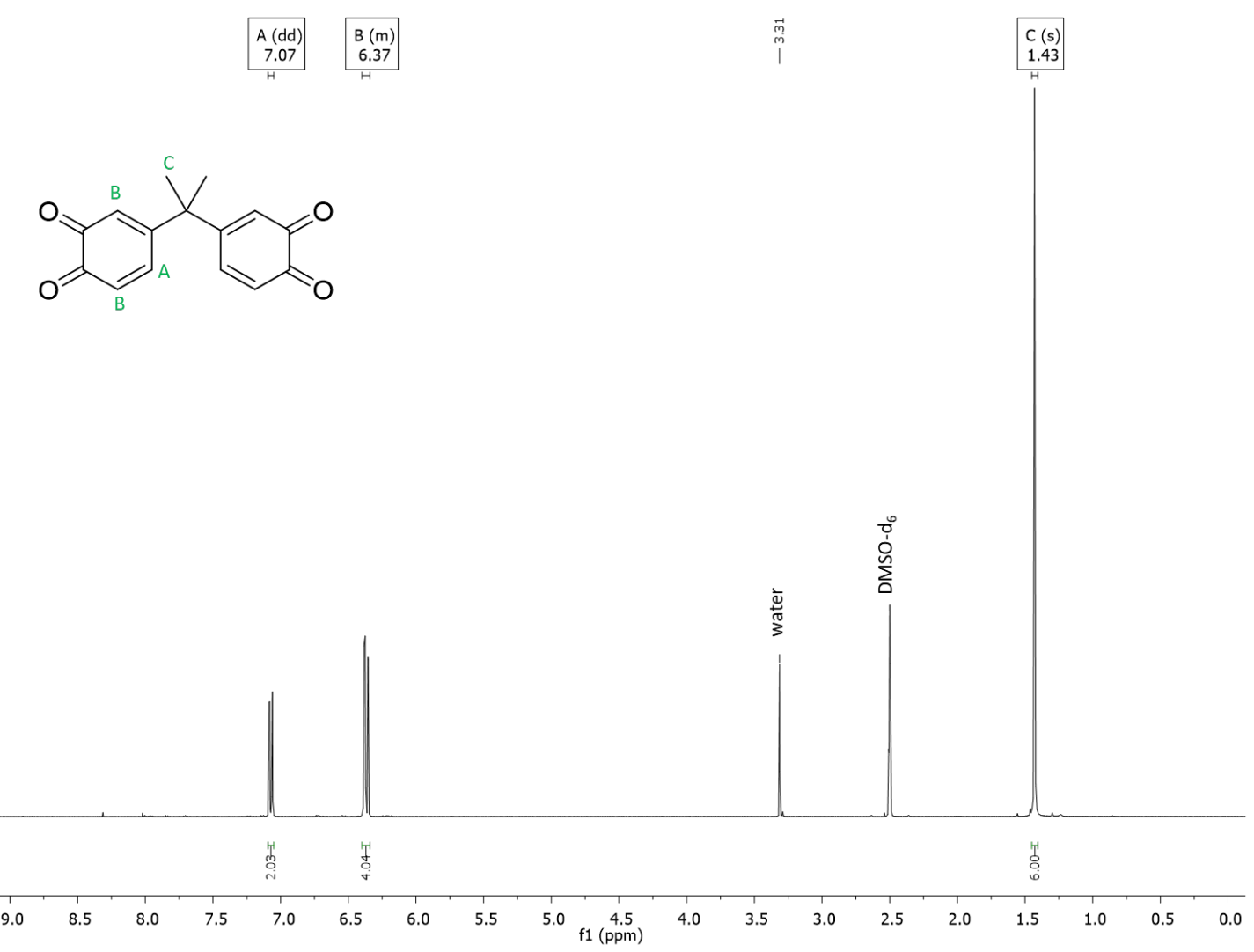

Figure $\mathrm{S} 2 .{ }^{1} \mathrm{H}-\mathrm{NMR}$ of bisquinone A in DMSO- $\mathrm{d}_{6}$.
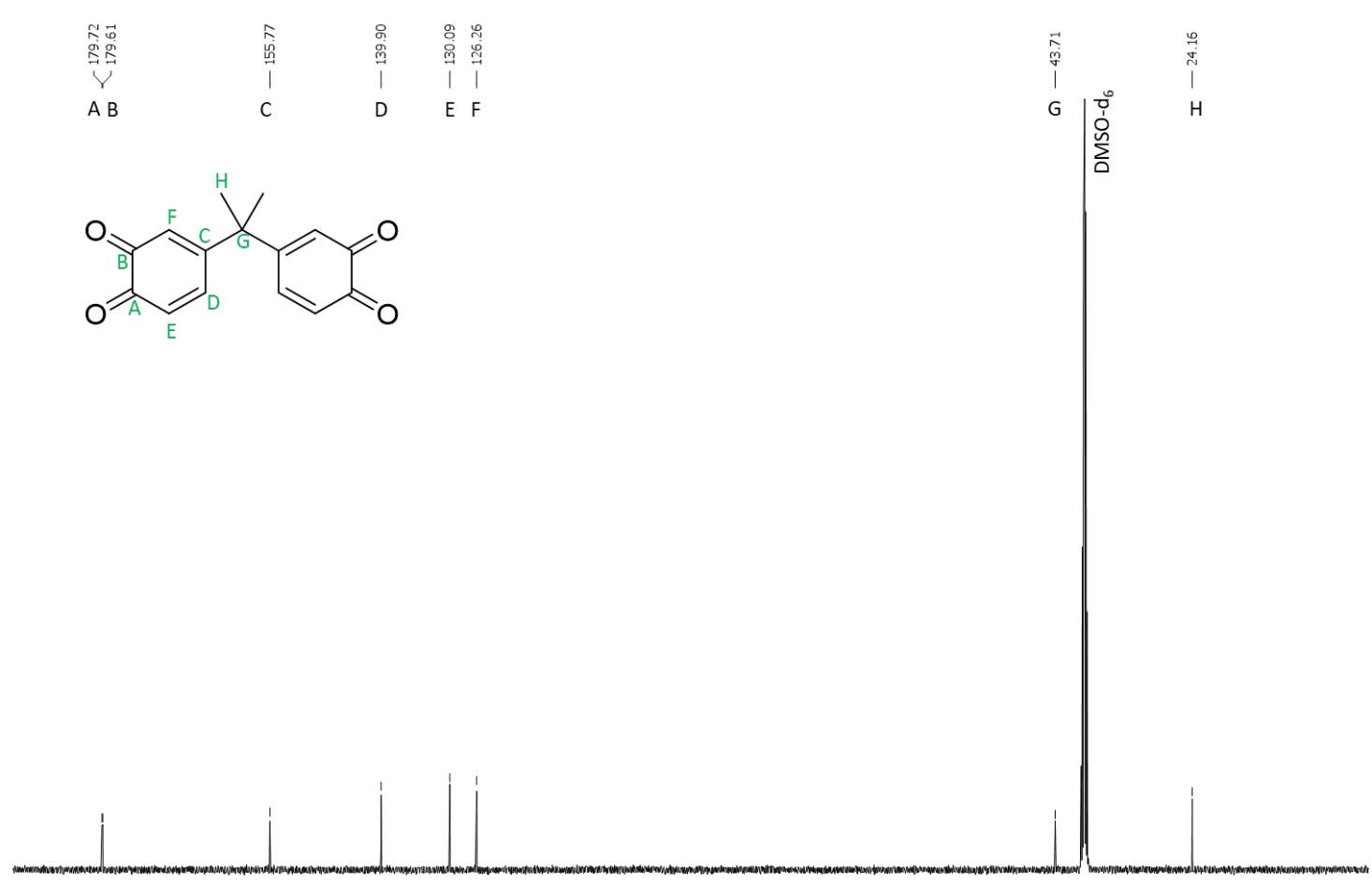

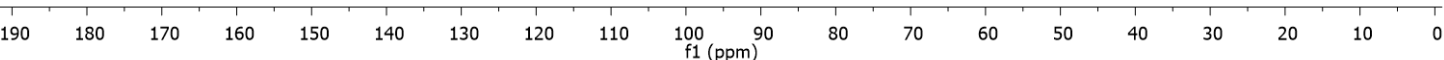

Figure $\mathrm{S} 3 .{ }^{13} \mathrm{C}-\mathrm{NMR}$ of bisquinone $\mathrm{A}$ in DMSO- $\mathrm{d}_{6}$. 


\subsection{Synthesis of bisquinone Z (BQZ)}<smiles>O=C1C=CC(C2(C3=CC(=O)C(=O)C=C3)CCCCC2)=CC1=O</smiles>

Bisphenol Z (6.0 g, $20 \mathrm{mmol})$ was dissolved in $200 \mathrm{~mL}$ methanol. The solution was mixed with IBX $(18.5 \mathrm{~g}, 66 \mathrm{mmol})$ and the mixture was stirred for $15 \mathrm{~min}$ at room temperature. Formation of a dark solid was observed. Subsequently, the mixture was cooled in an ice bath for $10 \mathrm{~min}$, the dark solid was filtrated and washed with cold methanol. The dark solid was redissolved in chloroform. After filtration, the organic solvent was removed under reduced pressure to obtain a brown solid in $4.9 \mathrm{~g}$ yield $(74 \%)$.

NMR spectra are shown in Figure S 4 and Figure S 5.

${ }^{1} \mathrm{H}-\mathrm{NMR}\left(500 \mathrm{MHz}, \mathrm{DMSO}-\mathrm{d}_{6}\right): \delta[\mathrm{ppm}] 7.13(\mathrm{dd}, \mathrm{J}=10.4,2.4 \mathrm{~Hz}, 1 \mathrm{H}), 6.46(\mathrm{~d}, \mathrm{~J}=1.9 \mathrm{~Hz}$, $1 \mathrm{H}), 6.37-6.33(\mathrm{~m}, 1 \mathrm{H}), 2.00-1.92(\mathrm{~m}, 2 \mathrm{H}), 1.48(\mathrm{dd}, \mathrm{J}=30.0,4.3 \mathrm{~Hz}, 3 \mathrm{H})$.

${ }^{13} \mathrm{C}-\mathrm{NMR}\left(126 \mathrm{MHz}, \mathrm{DMSO}-\mathrm{d}_{6}\right): \delta[\mathrm{ppm}] 179.76,179.53,153.43,139.38,130.06,128.51$, $46.95,30.97,25.18,21.53$.

FT-IR: 2978 (w), $2928(\mathrm{w}), 1684(\mathrm{~m}), 1661$ (s), $1622(\mathrm{~m}), 1562$ (m), 1465 (w), 1404 (m), 1281 (m), $1140(\mathrm{~m}), 1032(\mathrm{w}), 891(\mathrm{w}), 830(\mathrm{w}), 818(\mathrm{~m}), 800(\mathrm{~m}), 766(\mathrm{w}), 726(\mathrm{w}), 692(\mathrm{w}), 646$ (m), $613(\mathrm{~m})$.

\section{UPLC-UV/Vis-QMS:}

$\mathrm{UV} / \mathrm{Vis}(210 \mathrm{~nm}): \mathrm{t}_{\mathrm{R}}=2.643 \mathrm{~min}$ und $2.761 \mathrm{~min}, 99 \%$ purity (solvent A (Milli-Q water with $0.1 \% \mathrm{FA}, \mathrm{v} / \mathrm{v}$ ) and solvent B (acetonitrile with 0.1\% FA), Gradient 10-90\% B).

ESI-QMS: calculated (m/z): 295.09 [M-H]', 591.19 [2M-H]-; found (m/z): 297.07 [M-H], $595.20[2 \mathrm{M}-\mathrm{H}]^{-}$. The mass differences of $+2 \mathrm{Da}$ and $+4 \mathrm{Da}$ found for the bisquinone monomer were consistent with the literature as discussed in detail in the bisquinone A analysis data section on page SI S4.1. 


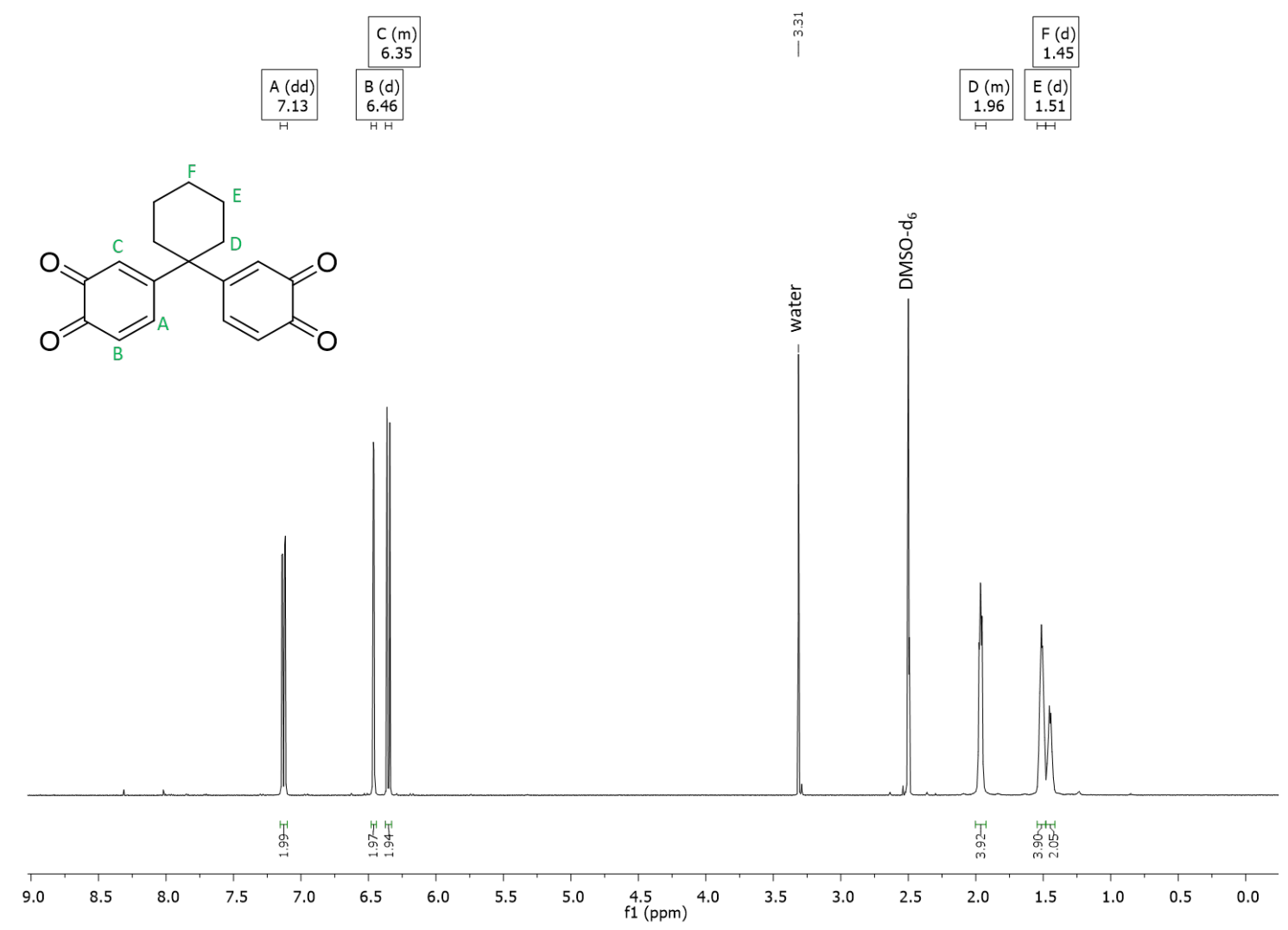

Figure $\mathrm{S} 4 .{ }^{1} \mathrm{H}-\mathrm{NMR}$ of bisquinone $\mathrm{Z}$ in DMSO-d .

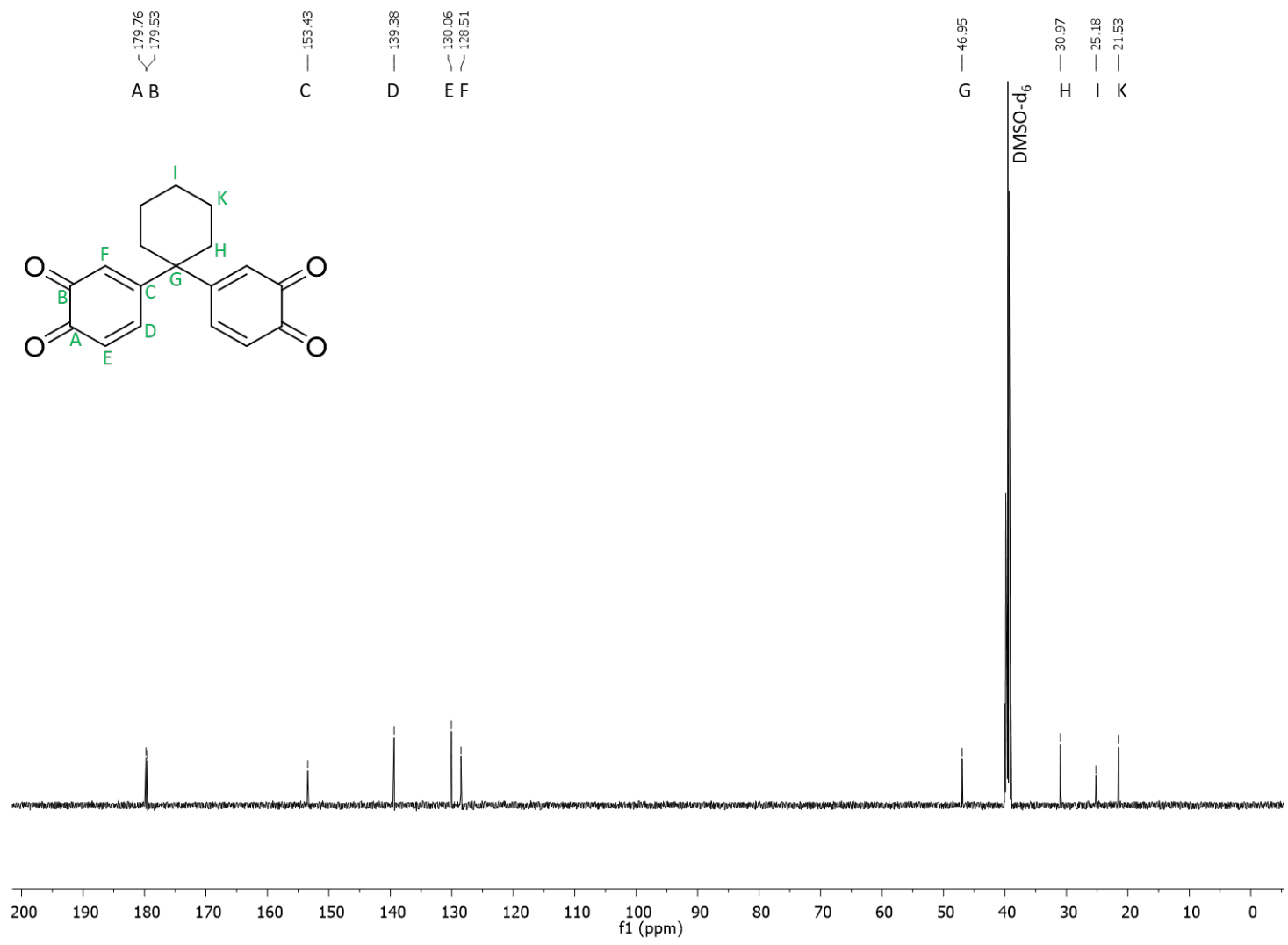

Figure $\mathrm{S} 5 .{ }^{13} \mathrm{C}-\mathrm{NMR}$ of bisquinone $\mathrm{Z}$ in DMSO- $\mathrm{d}_{6}$. 


\subsection{Synthesis of bisquinone B (BQB)}<smiles>CCC(C)(C1=CC(=O)C(=O)C=C1)C1=CC(=O)C(=O)C=C1</smiles>

Bisphenol B (5.3 g, $20 \mathrm{mmol})$ was dissolved in $200 \mathrm{~mL}$ methanol. The solution was mixed with IBX $(18.5 \mathrm{~g}, 66 \mathrm{mmol})$ and the mixture was stirred for $15 \mathrm{~min}$ at room temperature. Formation of a red solid was observed. Subsequently, the mixture was cooled in an ice bath for $10 \mathrm{~min}$, the solid was filtrated and washed with cold methanol. The red solid was redissolved in chloroform. After filtration, the organic solvent was removed under reduced pressure and a brown solid was obtained in $5.1 \mathrm{~g}$ yield $(85 \%)$.

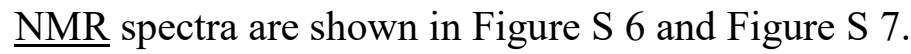

${ }^{1} \mathrm{H}-\mathrm{NMR}\left(500 \mathrm{MHz}, \mathrm{DMSO}-\mathrm{d}_{6}\right): \delta[\mathrm{ppm}] 7.03(\mathrm{dd}, \mathrm{J}=10.2,2.5 \mathrm{~Hz}, 2 \mathrm{H}), 6.38-6.31(\mathrm{~m}, 4 \mathrm{H})$, $1.87(\mathrm{q}, \mathrm{J}=7.4 \mathrm{~Hz}, 2 \mathrm{H}), 1.35(\mathrm{~s}, 3 \mathrm{H}), 0.82(\mathrm{t}, \mathrm{J}=7.4 \mathrm{~Hz}, 3 \mathrm{H})$.

${ }^{13} \mathrm{C}-\mathrm{NMR}\left(126 \mathrm{MHz}, \mathrm{DMSO}-\mathrm{d}_{6}\right): \delta[\mathrm{ppm}] 179.78,179.43,154.88,139.93,130.06,127.39$, $47.25,27.53,20.63,8.40$.

FT-IR: 2978 (w), $2928(\mathrm{w}), 1684(\mathrm{~m}), 1661$ (s), $1622(\mathrm{~m}), 1562(\mathrm{~m}), 1465(\mathrm{w}), 1404(\mathrm{~m}), 1281$ (m), $1140(\mathrm{~m}), 1032(\mathrm{w}), 891(\mathrm{w}), 830(\mathrm{w}), 818(\mathrm{~m}), 800(\mathrm{~m}), 766(\mathrm{w}), 726(\mathrm{w}), 692(\mathrm{w}), 646$ (m), $613(\mathrm{~m})$.

\section{UPLC-UV/Vis-QMS:}

$\mathrm{UV} / \mathrm{Vis}(210 \mathrm{~nm}): \mathrm{t}_{\mathrm{R}}=2.245 \mathrm{~min}, 2.430 \mathrm{~min}$ und $2.457 \mathrm{~min}, 99 \%$ purity (solvent A (Milli-Q water with $0.1 \% \mathrm{FA}, \mathrm{v} / \mathrm{v}$ ) and solvent B (acetonitrile with $0.1 \% \mathrm{FA}$ ), Gradient 10-90\% B). ESI-QMS: calculated (m/z): 269.08 [M-H] $^{-}, 539.17$ [2M-H]-; found (m/z): $271.05[\mathrm{M}-\mathrm{H}]^{-}$, $543.16[2 \mathrm{M}-\mathrm{H}]^{-}$. The mass differences of $+2 \mathrm{Da}$ and $+4 \mathrm{Da}$ found for the bisquinone monomer were consistent with the literature as discussed in detail in the bisquinone A analysis data section on page SI S4.1. 

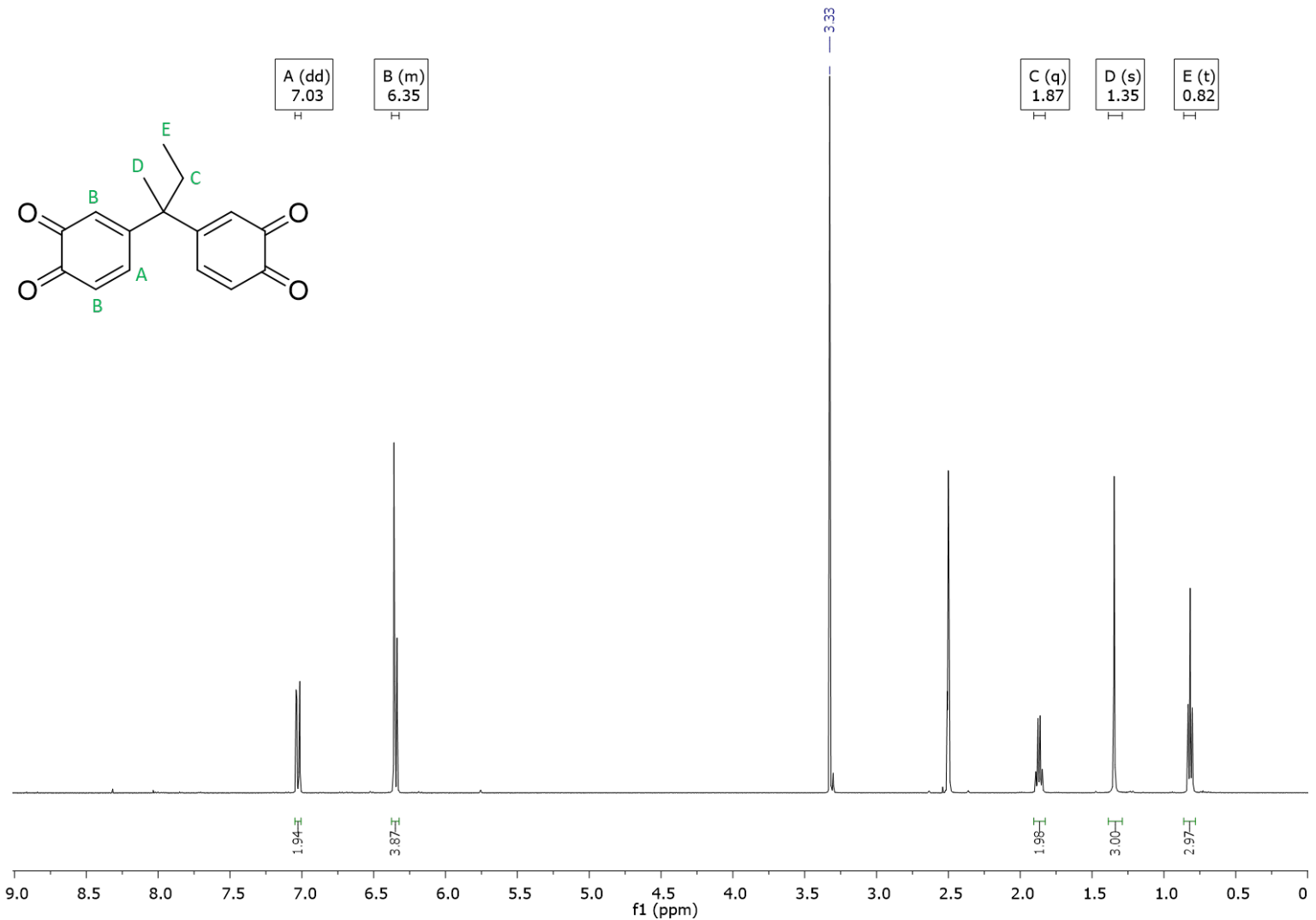

Figure S 6. ${ }^{1} \mathrm{H}-\mathrm{NMR}$ of bisquinone B in DMSO- $\mathrm{d}_{6}$.

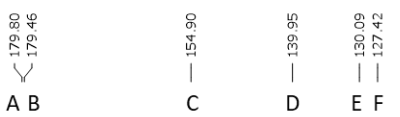<smiles>CCC(I)(C1=CC(=O)C(=O)C=C1)C1=CC(=O)C(=O)C=C1</smiles>

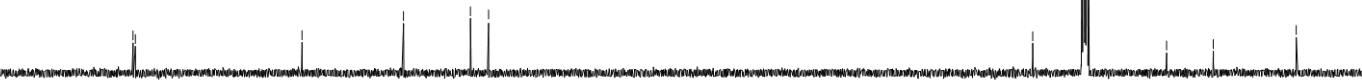

$\begin{array}{llllllllllllllllllll}190 & 180 & 170 & 160 & 150 & 140 & 130 & 120 & 110 & 100 & 90 & 80 & 70 & 60 & 50 & 40 & 30 & 20 & 10 & 0\end{array}$

Figure $\mathrm{S} 7 .{ }^{13} \mathrm{C}-\mathrm{NMR}$ of bisquinone $\mathrm{B}$ in DMSO- $\mathrm{d}_{6}$. 


\subsection{Synthesis of bisquinone M (BQM)}<smiles>CC(C)(C1=CC(=O)C(=O)C=C1)c1cccc(C(C)(C)C2=CC(=O)C(=O)C=C2)c1</smiles>

Bisphenol M (1.0 g, $2.9 \mathrm{mmol})$ was dissolved in $70 \mathrm{~mL}$ methanol. The solution was mixed with $\operatorname{IBX}(2.4 \mathrm{~g}, 8.7 \mathrm{mmol})$ and the mixture was stirred for $30 \mathrm{~min}$ at room temperature. The colored reaction mixture was filtered, and the filtrate was precipitated in $1 \mathrm{~L}$ phosphate buffer $(0.5 \mathrm{M}$, pH 6.5). The precipitate was filtered off, was washed with Milli-Q water with 5\% formic acid and was dried. An orange solid was obtained in $0.92 \mathrm{~g}$ yield (85\%).

NMR spectra are shown in Figure S 8 and Figure S 9.

${ }^{1} \mathrm{H}-\mathrm{NMR}\left(500 \mathrm{MHz}, \mathrm{DMSO}-\mathrm{d}_{6}\right): \delta[\mathrm{ppm}] 7.37-7.31(\mathrm{~m}, 1 \mathrm{H}), 7.30-7.22(\mathrm{~m}, 3 \mathrm{H}), 6.68(\mathrm{dd}, \mathrm{J}$ $=10.3,2.3 \mathrm{~Hz}, 2 \mathrm{H}), 6.38(\mathrm{~d}, \mathrm{~J}=2.3 \mathrm{~Hz}, 2 \mathrm{H}), 6.22(\mathrm{~d}, \mathrm{~J}=10.3 \mathrm{~Hz}, 2 \mathrm{H}), 1.51(\mathrm{~s}, 12 \mathrm{H})$.

${ }^{13} \mathrm{C}-\mathrm{NMR}\left(126 \mathrm{MHz}, \mathrm{DMSO}-\mathrm{d}_{6}\right): \delta[\mathrm{ppm}] 180.05,179.98,159.66,145.17,141.03,129.04$, $125.17,124.79,124.08,43.38,26.90$.

FT-IR: 2972 (m), 2936 (w), 1684 (m), 1663 (s), 1622 (m), 1568 (m), 1489 (w), 1464 (w), 1394 (m), $1369(\mathrm{w}), 1302(\mathrm{w}), 1279(\mathrm{~m}), 1219(\mathrm{w}), 1134(\mathrm{~m}), 1047(\mathrm{w}), 893(\mathrm{w}), 820(\mathrm{~m}), 716(\mathrm{~m})$, $648(w), 630(w), 596(w)$.

\section{UPLC-UV/Vis-QMS:}

$\mathrm{UV} / \mathrm{Vis}(210 \mathrm{~nm}): \mathrm{t}_{\mathrm{R}}=3.366 \mathrm{~min}, 98 \%$ purity (solvent A (Milli-Q water with $0.1 \% \mathrm{FA}, \mathrm{v} / \mathrm{v}$ ) and solvent B (acetonitrile with 0.1\% FA), Gradient 10-90\% B).

ESI-QMS: calculated (m/z): $373.14[\mathrm{M}-\mathrm{H}]^{-}$, found $(\mathrm{m} / \mathrm{z}): 375.19[\mathrm{M}-\mathrm{H}]^{-}$. The mass differences of $+2 \mathrm{Da}$ and $+4 \mathrm{Da}$ found for the bisquinone monomer were consistent with the literature as discussed in detail in the bisquinone A analysis data section on page SI S4.1. 


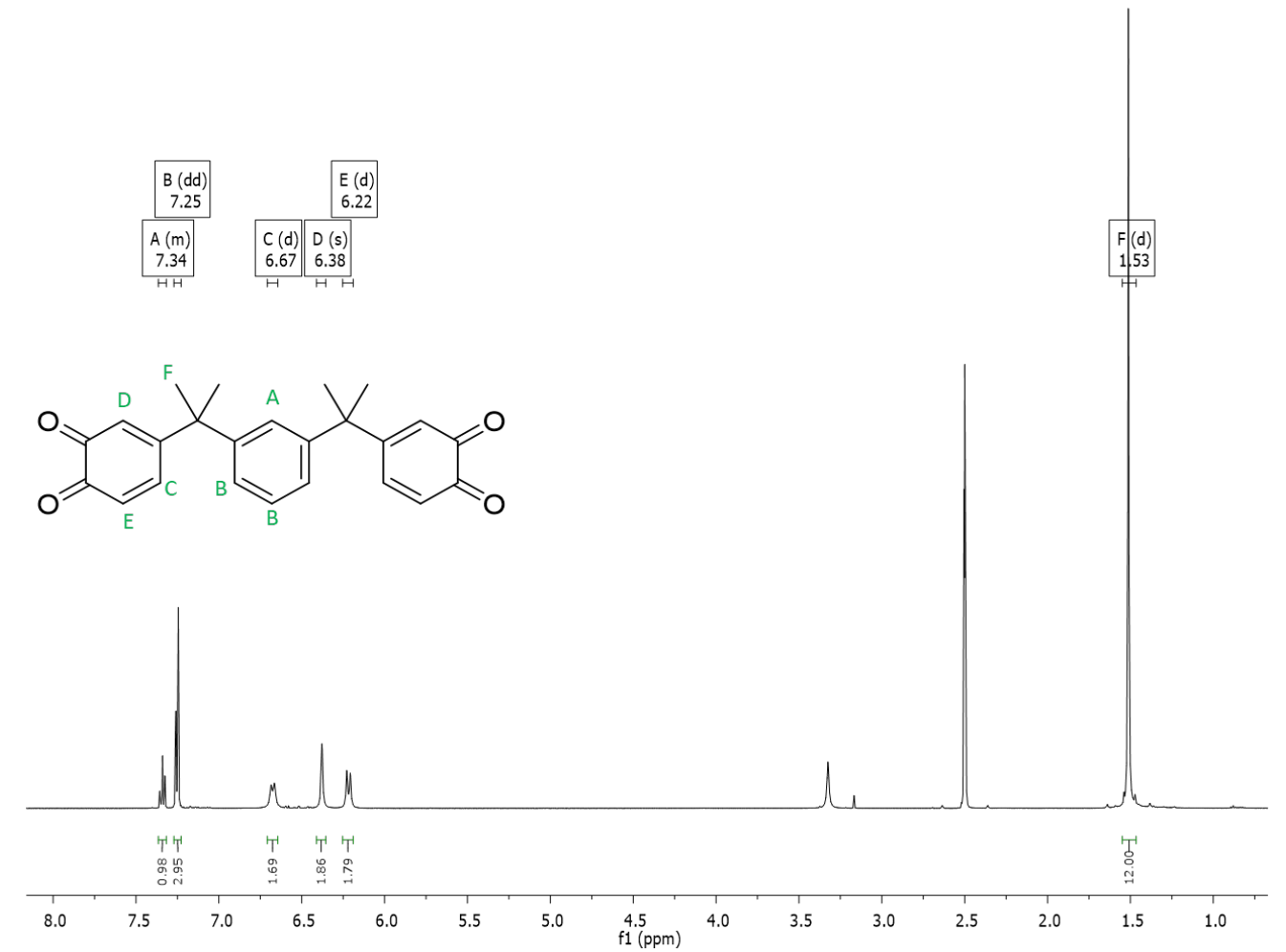

Figure S 8. ${ }^{1} \mathrm{H}-\mathrm{NMR}$ of bisquinone M in DMSO- $\mathrm{d}_{6}$.
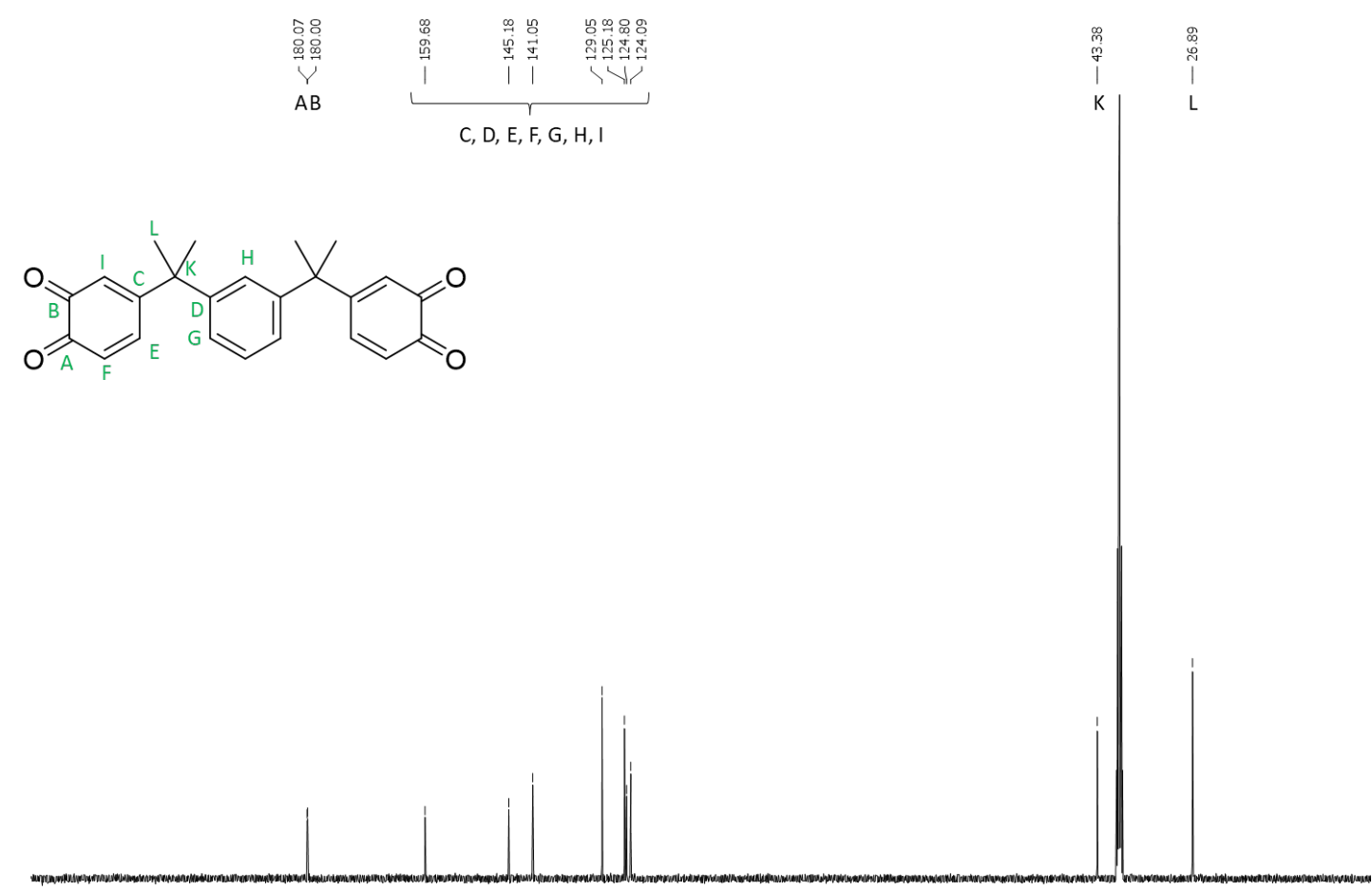

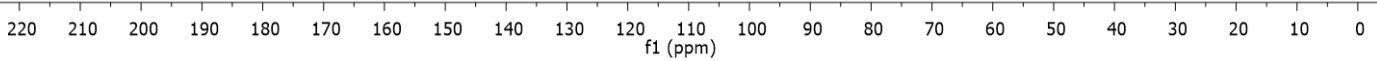

Figure S 9. ${ }^{13} \mathrm{C}-\mathrm{NMR}$ of bisquinone $\mathrm{M}$ in DMSO- $\mathrm{d}_{6}$. 


\subsection{Synthesis of bisquinone FL (BQFL)}<smiles>O=C1C=CC(C2(C3=CCC(=O)C(=O)C3)c3ccccc3-c3ccccc32)=CC1=O</smiles>

Bisphenol FL (1.0 g, $2.6 \mathrm{mmol})$ was dissolved in $70 \mathrm{~mL}$ methanol. The solution was mixed with IBX (2.4 g, $8.7 \mathrm{mmol})$ and the mixture was stirred for $30 \mathrm{~min}$ at room temperature. The colored reaction mixture was filtered, and the filtrate was precipitated in $1 \mathrm{~L}$ phosphate buffer (0.5 M, pH 6.5). The precipitate was separated by centrifugation, was washed with Milli-Q water with 5\% formic acid and was dried. An orange solid was obtained in $0.96 \mathrm{~g}$ yield (89\%).

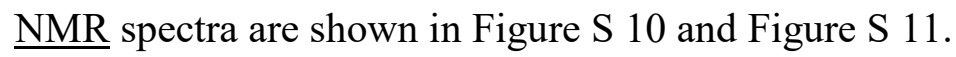

${ }^{1} \mathrm{H}-\mathrm{NMR}\left(500 \mathrm{MHz}, \mathrm{DMSO}-\mathrm{d}_{6}\right): \delta[\mathrm{ppm}] 8.07(\mathrm{dt}, \mathrm{J}=7.7,0.9 \mathrm{~Hz}, 1 \mathrm{H}), 7.67(\mathrm{dt}, \mathrm{J}=7.7,0.9 \mathrm{~Hz}$, 1H), $7.58(\mathrm{td}, \mathrm{J}=7.5,1.1 \mathrm{~Hz}, 1 \mathrm{H}), 7.47(\mathrm{td}, \mathrm{J}=7.5,1.2 \mathrm{~Hz}, 1 \mathrm{H}), 6.92(\mathrm{dd}, \mathrm{J}=10.4,2.4 \mathrm{~Hz}$, $1 \mathrm{H}), 6.36(\mathrm{dd}, \mathrm{J}=10.4,0.7 \mathrm{~Hz}, 1 \mathrm{H}), 6.13(\mathrm{dd}, \mathrm{J}=2.4,0.7 \mathrm{~Hz}, 1 \mathrm{H})$.

${ }^{13} \mathrm{C}-\mathrm{NMR}\left(126 \mathrm{MHz}, \mathrm{DMSO}-\mathrm{d}_{6}\right): \delta[\mathrm{ppm}] 179.30,179.23,151.47,142.77,140.88,139.49$, $130.49,129.79,128.56,127.55,126.10,121.53,63.56$.

FT-IR: 3061 (w), $1685(\mathrm{~m}), 1663(\mathrm{~s}), 1620$ (m), $1560(\mathrm{~m}), 1448(\mathrm{~m}), 1396(\mathrm{~m}), 1269$ (m), 1169 (w), $1136(\mathrm{w}), 1124(\mathrm{w}), 800(\mathrm{~m}), 752(\mathrm{~m}), 733(\mathrm{~m}), 961(\mathrm{w}), 676(\mathrm{w}), 654(\mathrm{w}), 606(\mathrm{w})$.

$\mathrm{UV} / \mathrm{Vis}(210 \mathrm{~nm}): \mathrm{t}_{\mathrm{R}}=3.148 \mathrm{~min}$ und $3.260 \mathrm{~min}, 93 \%$ purity (solvent A (Milli-Q water with $0.1 \%$ FA, v/v) and solvent B (acetonitrile with 0.1\% FA), Gradient 10-90\% B).

ESI-QMS: calculated (m/z): 377.08 [M-H]', 754.07 [2M-H]-; found (m/z): $379.14[\mathrm{M}-\mathrm{H}]^{-}$, $759.21[2 \mathrm{M}-\mathrm{H}]^{-}$. The mass differences of $+2 \mathrm{Da}$ and $+4 \mathrm{Da}$ found for the bisquinone monomer were consistent with the literature as discussed in detail in the bisquinone A analysis data section on page SI S4.1. 


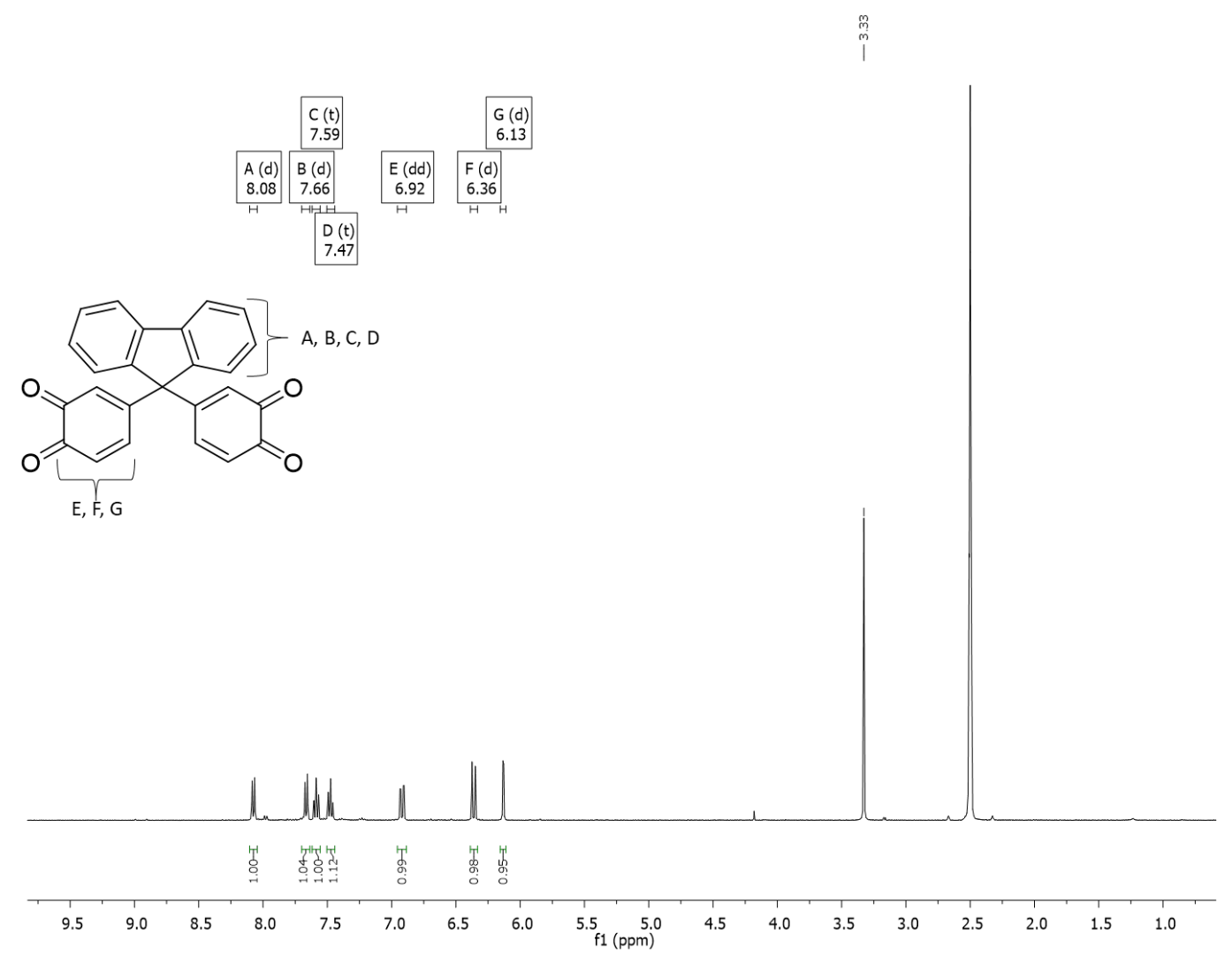

Figure S $10 .{ }^{1} \mathrm{H}-\mathrm{NMR}$ of bisquinone FL in DMSO- $\mathrm{d}_{6}$.
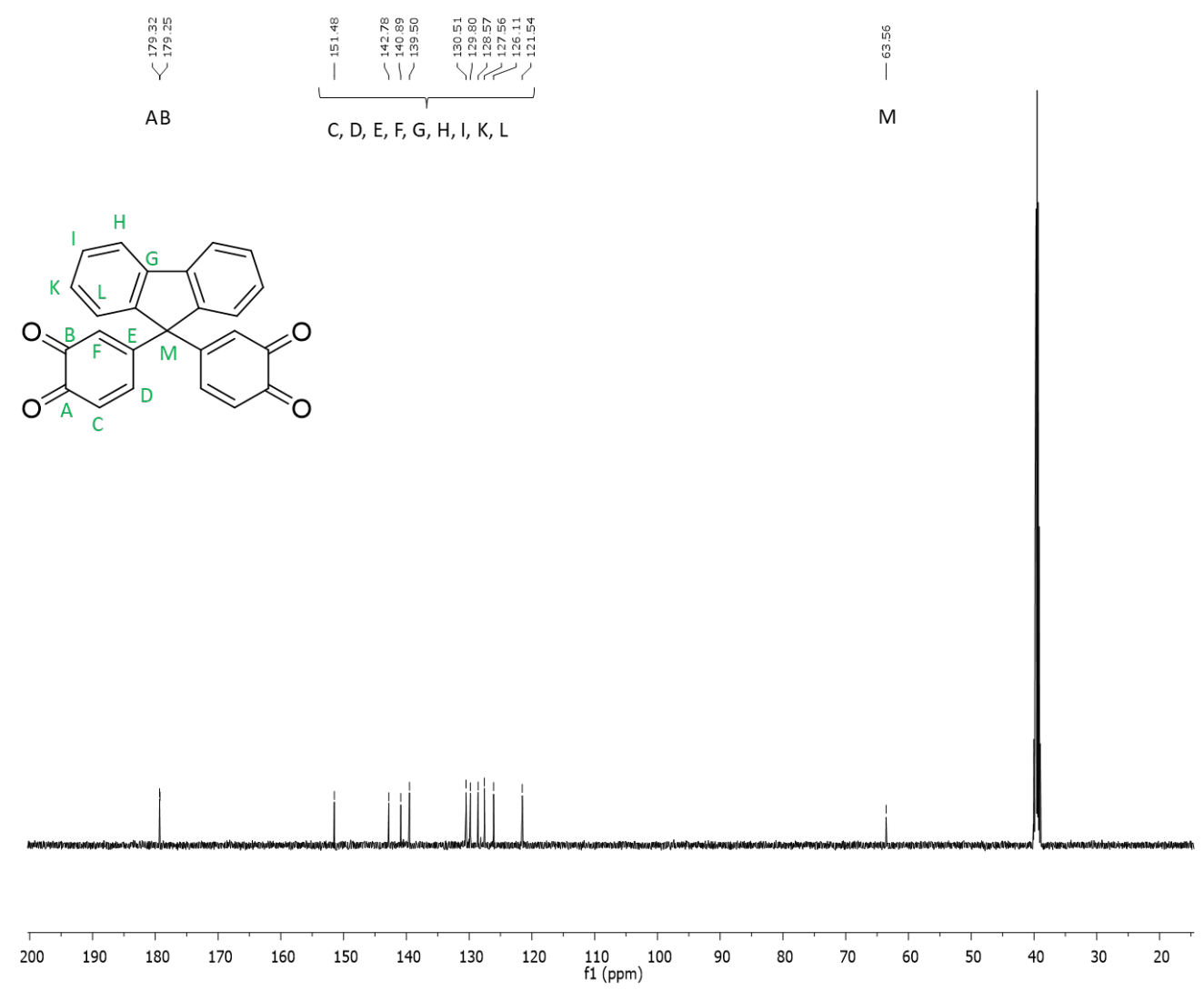

Figure S $11 .{ }^{13} \mathrm{C}-\mathrm{NMR}$ of bisquinone FL in DMSO-d $\mathrm{d}_{6}$. 


\section{Synthesis of the Polymer Matrix}

\subsection{Investigation of the precipitation procedure}

Precipitation conditions have been investigated for selected monomer combinations (BQA with various dithiols as well as EDET with different BQs), whereby GPC data (Figure S 12 to Figure S 16) and yield (Table S2) have been considered. It was found that the suitable methanol concentration for precipitation depends on the combined monomers and the appropriate range of the methanol proportion extends from $20 \%$ and $35 \%$ methanol for $\mathrm{p}$ (BQA-DTT) and $\mathrm{p}$ (BQADMP) to $65 \%$ in the case of $\mathrm{p}(\mathrm{BQA}-\mathrm{HDT})$. Based on these results precipitation conditions have been transferred to the whole polymer matrix.

Therefore BQA $(90.4 \mathrm{mg}, 0.353 \mathrm{mmol})$ was polymerized with 1 equiv. of the dithiols EDET (60.4 $\mu \mathrm{L}), \operatorname{BMT}(61.3 \mathrm{mg})$, DTT (55.5 mg), DMP (37.2 $\mu \mathrm{L}), \operatorname{GDMA}(53.4 \mu \mathrm{L}), \operatorname{BDT}(42.7 \mu \mathrm{L})$ as well as HDT $(56.2 \mu \mathrm{L})$ in $4 \mathrm{~mL}$ NMP. Furthermore, TCC-polymers from BQZ (104.5 mg), BQM (132.1 mg) and BQFL (133.5 mg) with EDET (60.4 $\mu \mathrm{L})$ were synthesized in $4 \mathrm{~mL}$ NMP. Aliquots of $500 \mu \mathrm{L}$ were precipitated in different solutions (4 mL) according to Table S 1. Residues were dried, weight and solved in $10 \mathrm{~mL}$ THF for GPC analysis. Resulting mass distributions of the GPC chromatograms are shown in Figure S 12 to Figure S 16.

Table S 1. Composition of precipitation solutions to investigate precipitation conditions.

\begin{tabular}{|c|c|c|c|c|}
\hline $\begin{array}{c}\text { precipitation } \\
\text { sample }\end{array}$ & $\begin{array}{r}\text { methanol } \\
{[\%]}\end{array}$ & $\begin{array}{c}\text { methanol } \\
{[\mathrm{mL}]}\end{array}$ & $\begin{array}{c}\text { water } \\
{[\%]}\end{array}$ & $\begin{array}{c}\text { water } \\
{[\mathrm{mL}]}\end{array}$ \\
\hline a & 80 & 3,2 & 20 & 0,8 \\
\hline b & 65 & 2,6 & 35 & 1,4 \\
\hline c & 50 & 2,0 & 50 & 2,0 \\
\hline d & 35 & 1,4 & 65 & 2,6 \\
\hline e & 20 & 0,8 & 80 & 3,2 \\
\hline f & 0 & 0 & 100 & 4,0 \\
\hline
\end{tabular}


Table S 2. Yields obtained from precipitation experiments for TCC-polymers in aqueous methanol. Yields above $100 \%$ could occur due to NMP residues because just one precipitation step was carried out.

\begin{tabular}{|c|c|c|c|c|c|c|c|}
\hline \multicolumn{7}{|c|}{ Yields obtained after precipitation in aqueous methanol } \\
\hline & \multicolumn{7}{|c|}{ (proportion of methanol in \%) } \\
\hline TCC-polymer & $80 \%$ & $65 \%$ & $50 \%$ & $35 \%$ & $\mathbf{2 0} \%$ & $0 \%$ \\
\hline p(BQA-EDET) & 9 & 68 & 98 & 112 & 122 & 113 \\
\hline p(BQA-BMT) & 20 & 89 & 108 & 116 & 126 & 143 \\
\hline p(BQA-DTT) & 12 & 9 & 21 & 73 & 100 & 107 \\
\hline p(BQA-DMP) & 6 & 6 & 34 & 85 & 101 & 115 \\
\hline p(BQA-GDMA) & 8 & 50 & 88 & 97 & 83 & 70 \\
\hline p(BQA-BDT) & 6 & 14 & 102 & 103 & 95 & 107 \\
\hline p(BQA-HDT) & 8 & 64 & 112 & 124 & 116 & 123 \\
\hline p(BQZ-EDET) & 36 & 88 & 102 & 107 & 105 & 110 \\
\hline p(BQM-EDET) & 70 & 89 & 94 & 98 & 98 & 90 \\
\hline p(BQFL-EDET) & 48 & 76 & 86 & 101 & 104 & 112 \\
\hline
\end{tabular}



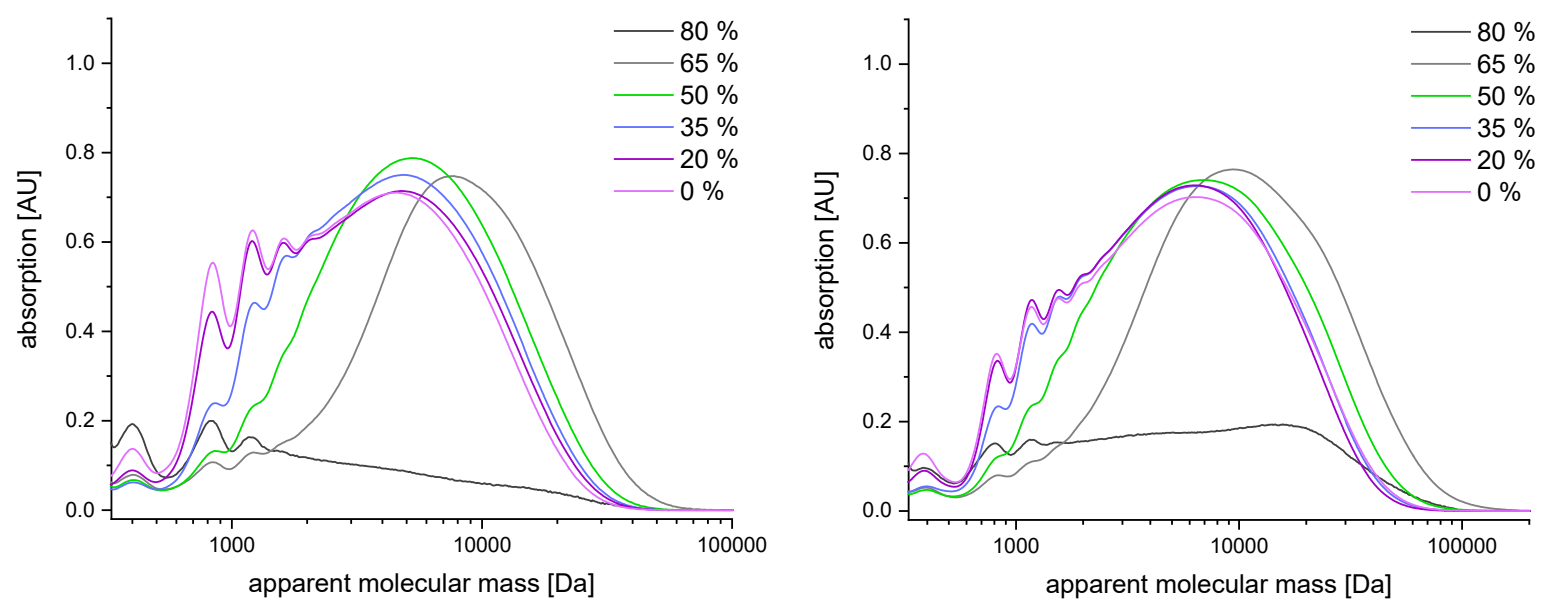

Figure S 12. Mass distributions of the GPC chromatograms (at $280 \mathrm{~nm}$ ) of the investigation of the precipitation procedure $\mathrm{p}(\mathrm{BQA}-\mathrm{EDET})$ (left) as well as $\mathrm{p}(\mathrm{BQA}-\mathrm{BMT})$ (right) in different aqueous solutions with varying methanol content.
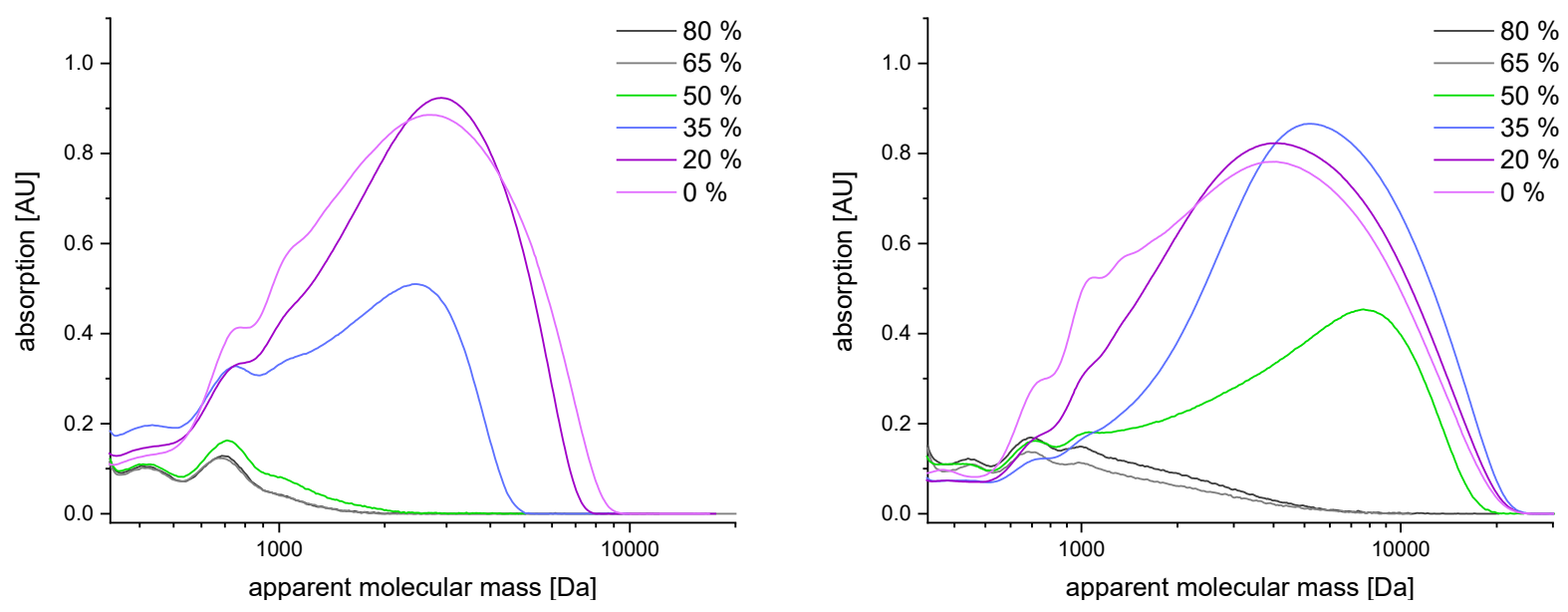

Figure S 13. Mass distributions of the GPC chromatograms (at $280 \mathrm{~nm}$ ) of the investigation of the precipitation procedure $\mathrm{p}(\mathrm{BQA}-\mathrm{DTT}$ ) (left) as well as $\mathrm{p}$ (BQA-DMP) (right) in different aqueous solutions with varying methanol content.
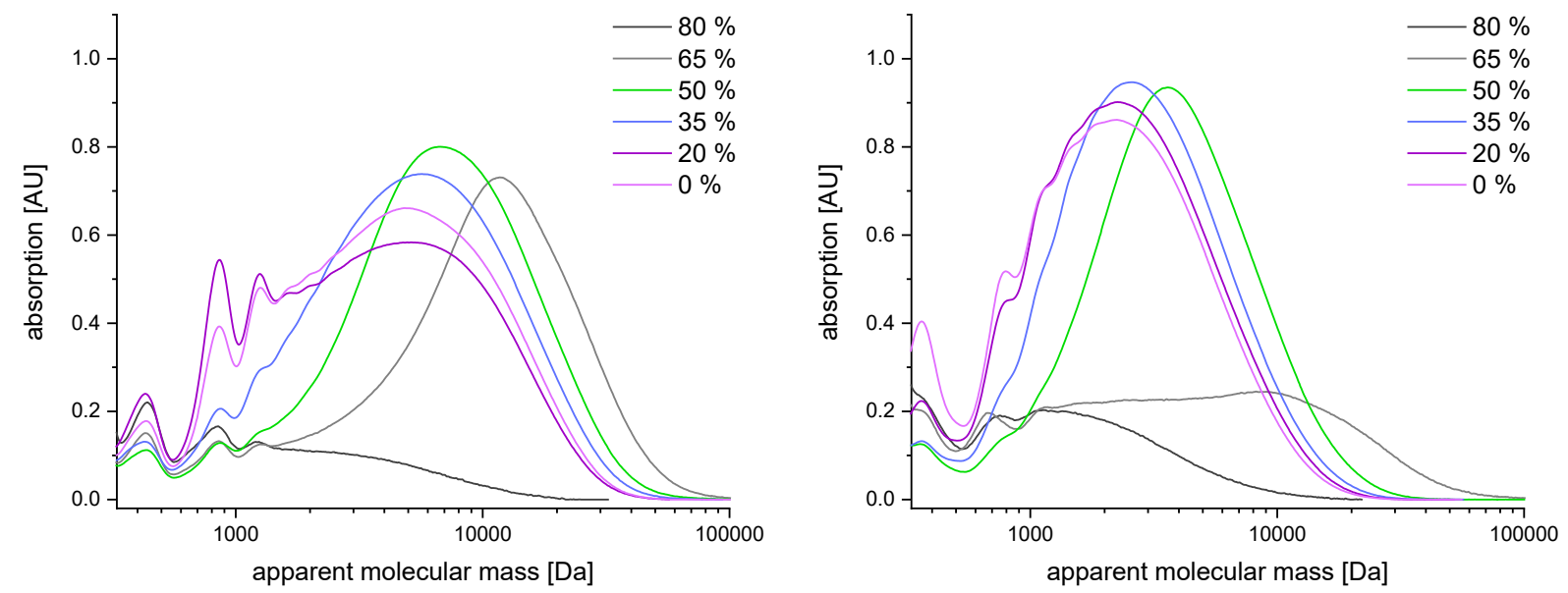

Figure S 14. Mass distributions of the GPC chromatograms (at $280 \mathrm{~nm}$ ) of the investigation of the precipitation procedure $\mathrm{p}(\mathrm{BQA}-\mathrm{GDMA})$ (left) as well as $\mathrm{p}(\mathrm{BQA}-\mathrm{BDT})$ (right) in different aqueous solutions with varying methanol content. 

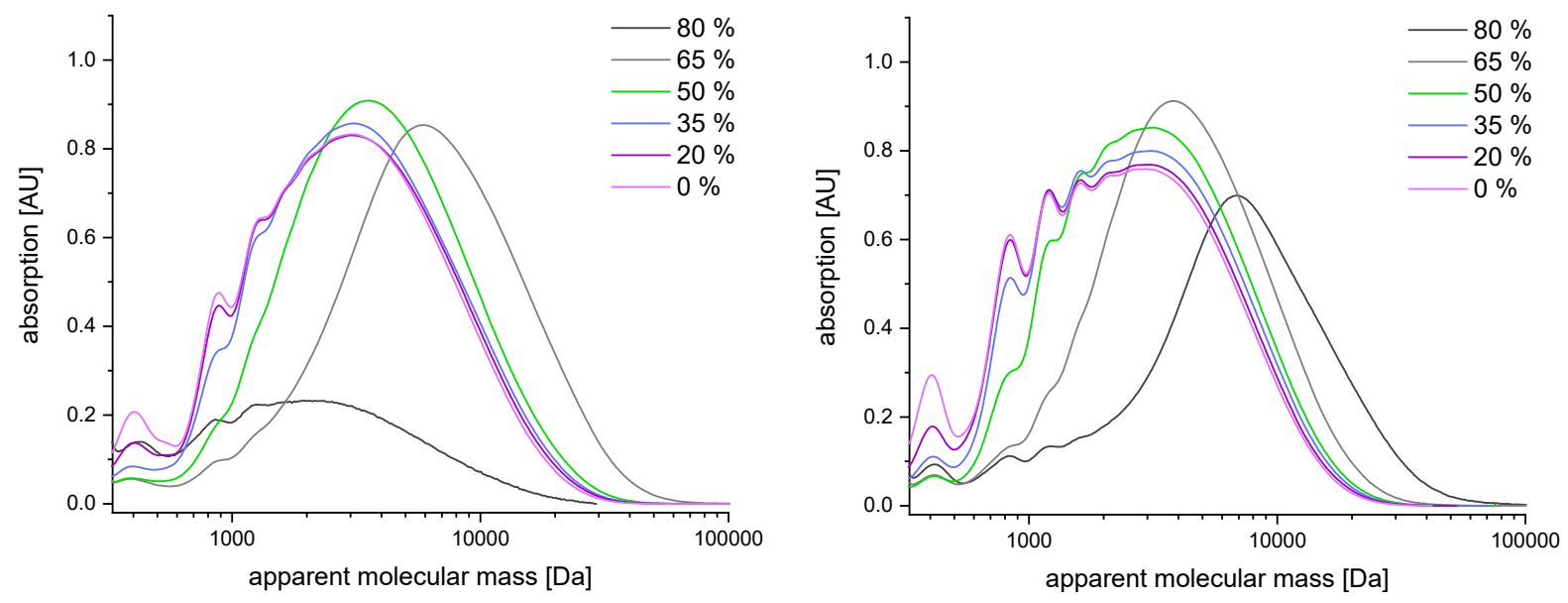

Figure S 15. Mass distributions of the GPC chromatograms (at $280 \mathrm{~nm}$ ) of the investigation of the precipitation procedure $\mathrm{p}(\mathrm{BQA}-\mathrm{HDT})$ (left) as well as $\mathrm{p}(\mathrm{BQZ}-\mathrm{EDET})$ (right) in different aqueous solutions with varying methanol content.
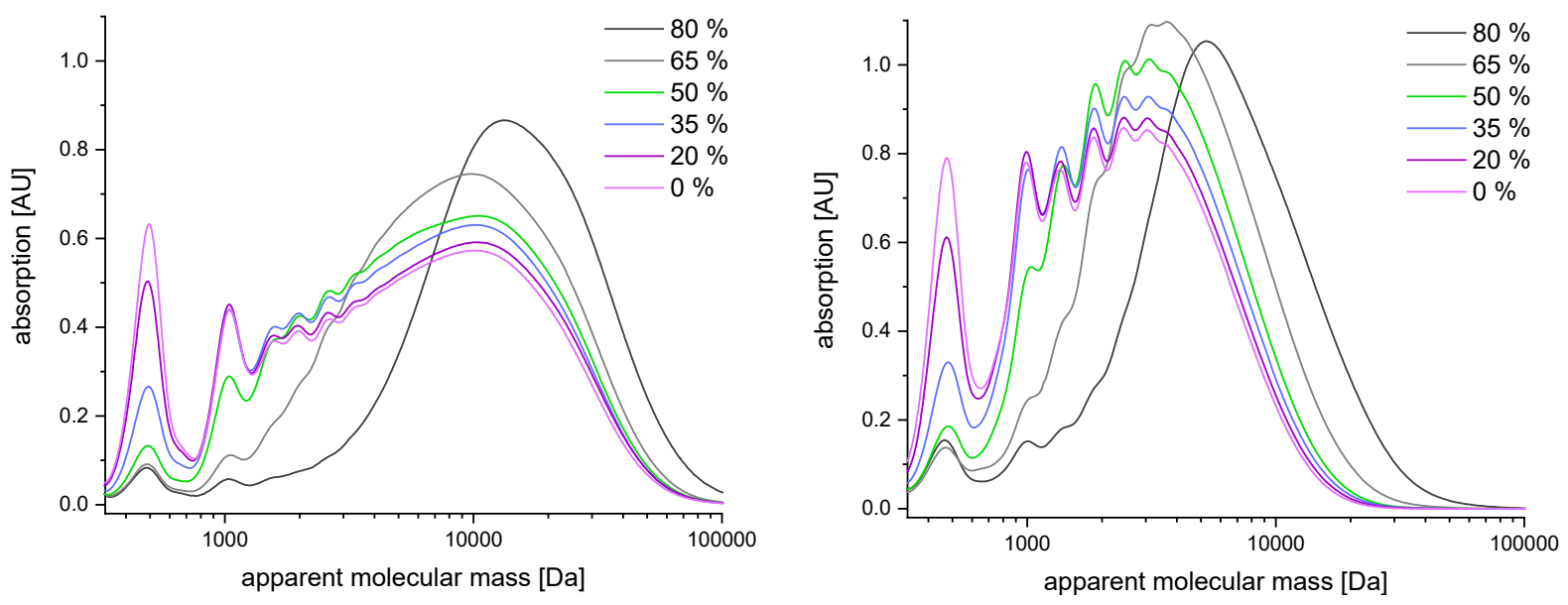

Figure S 16. Mass distributions of the GPC chromatograms (at $280 \mathrm{~nm}$ ) of the investigation of the precipitation procedure $\mathrm{p}(\mathrm{BQM}-\mathrm{EDET})$ (left) as well as $\mathrm{p}(\mathrm{BQFL}-\mathrm{EDET})$ (right) in different aqueous solutions with varying methanol content. 


\subsection{General polymerization procedure}

Polymerization was carried out for $15 \mathrm{~min}$ at room temperature. For this the bisquinone $(1.5 \mathrm{mmol})$ was solved in NMP $(10 \mathrm{~mL})$, the solution was stirred and one equivalent of the dithiol was added. After the reaction time the reaction mixture was precipitated in $120 \mathrm{~mL}$ in a $\mathrm{MeOH} / \mathrm{Milli}-\mathrm{Q}$ water mixture according to Table S 3, the precipitate was solved in acetone and precipitated in $120 \mathrm{~mL}$ Milli-Q water. The precipitate was isolated and dried.

Table S 3. MeOH proportion in the precipitation media for the first precipitation of the respective TCC-polymer synthesis.

\begin{tabular}{|c|c|c|c|c|c|}
\hline & BQA & BQZ & BQB & BQM & BQFL \\
\hline EDET & $60 \%$ & $60 \%$ & $60 \%$ & $60 \%$ & $60 \%$ \\
\hline BMT & $60 \%$ & $60 \%$ & $60 \%$ & $60 \%$ & $60 \%$ \\
\hline DTT & $20 \%$ & $20 \%$ & $20 \%$ & $20 \%$ & $20 \%$ \\
\hline DMP & $35 \%$ & $35 \%$ & $35 \%$ & $35 \%$ & $35 \%$ \\
\hline GDMA & $50 \%$ & $50 \%$ & $50 \%$ & $50 \%$ & $50 \%$ \\
\hline GDMA & $50 \%$ & $50 \%$ & $50 \%$ & $50 \%$ & $50 \%$ \\
\hline BDT & $50 \%$ & $50 \%$ & $50 \%$ & $50 \%$ & $50 \%$ \\
\hline HDT & $65 \%$ & $65 \%$ & $65 \%$ & $65 \%$ & $65 \%$ \\
\hline
\end{tabular}




\subsection{Polymer analysis}

\section{p(BQA-EDET)}<smiles>CC(C)(C)c1cc(O)c(O)c(SCCOCCOCCSc2cc(C(C)(C)C3=CC(=O)C(=O)C=C3)cc(O)c2O)c1</smiles>

Yield

$47 \%$

GPC

$\mathrm{M}_{w, \text { app }}=11.5 \mathrm{~kg} / \mathrm{mol}, \mathrm{Ð}=2.1, \mathrm{DP}(\mathrm{AA}+\mathrm{BB})=26$

$\mathrm{T}_{\mathrm{g}}$

$68^{\circ} \mathrm{C}$

TGA

$339{ }^{\circ} \mathrm{C}$ (maximal degradation rate)

${ }^{1} \mathrm{H}-\mathrm{NMR}(500 \mathrm{MHz}) \quad \delta[\mathrm{ppm}] 9.40-8.10$ (m, br.; 4H; BQ ArOH), 7.03 - 6.22 (m, br.; 4H; in $\mathrm{DMSO}^{-\mathrm{d}_{6}}$ BQ ArH), 3.69-2.75 (m, br.; 13H; DT aliphatic H; integral includes signal of $\mathrm{H}_{2} \mathrm{O}$ ), 1.83 - 1.38 (m, br.; $6 \mathrm{H}$; BQ aliphatic $\mathrm{H}$ ).

FT-IR $\left[\mathrm{cm}^{-1}\right]$ 3326 (s), 2964 (m), 2929 (m), 2871 (m), 1700 (w), 1590 (m), 1478 (s), 1412 (m), 1354 (m), 1272 (s), 1230 (s), 1200 (s), 1152 (s), 1132 (s), 1092 (s), 973 (m), $954(\mathrm{~m}), 908$ (w), $866(\mathrm{w}), 814(\mathrm{w}), 786(\mathrm{w})$, $734(w), 648$ (w).

MALDI-TOF-MS calculated $(\mathrm{m} / \mathrm{z}):[\mathrm{M}(4 \mathrm{AA}+4 \mathrm{BB})+\mathrm{Na}]^{+}=1777.5$ found $(\mathrm{m} / \mathrm{z}):[\mathrm{M}(4 \mathrm{AA}+4 \mathrm{BB})+\mathrm{Na}]^{+}=1777.4$
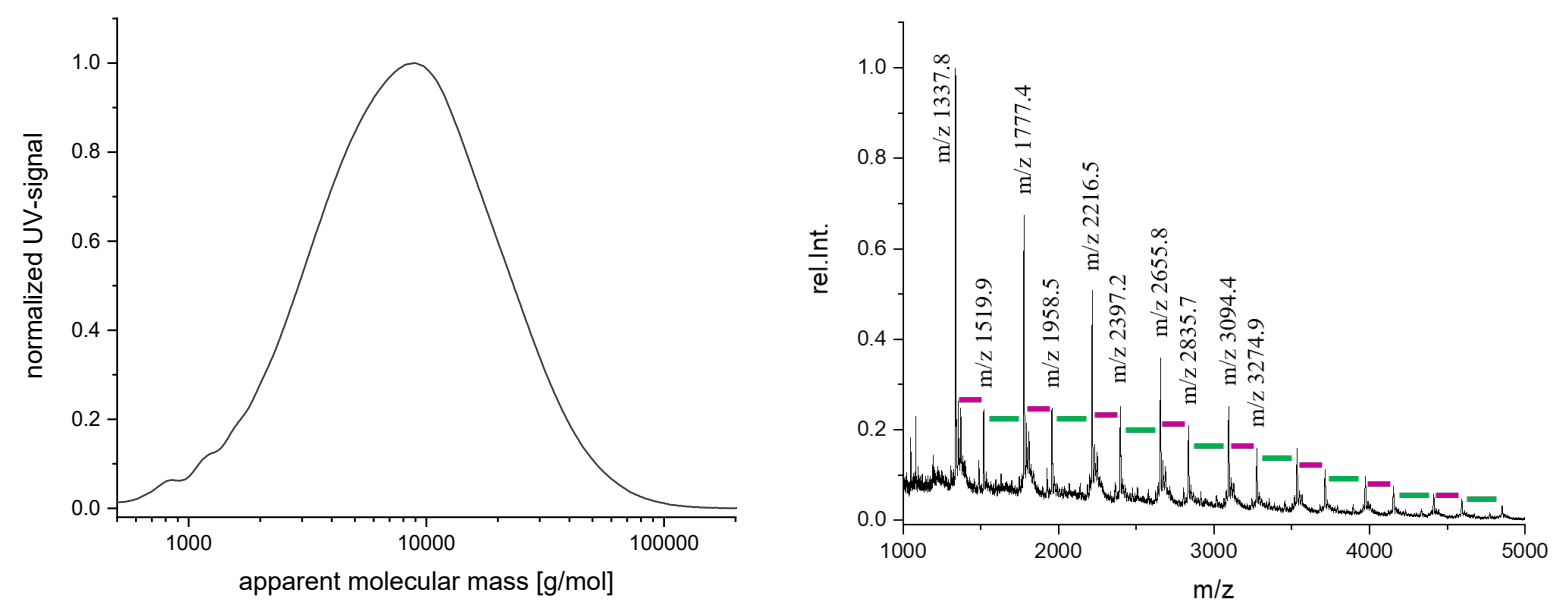

Figure S 17. p(BQA-EDET) analysis. GPC chromatogram at $280 \mathrm{~nm}$ (left) and MALDI-TOF-MS spectrum (right, $\mathrm{m} / \mathrm{z}$ values were assigned to $\mathrm{Na}^{+}$ion adducts of the oligomer showing the homologous raw of $\mathrm{AA}$ and $\mathrm{BB}$ monomer additions in the TCC-polymer product. Colored bars represent the $\mathrm{m} / \mathrm{z}$ differences corresponding to the repeating units, where green refers to biscatechols and purple to dithiols). 
<smiles>CC(C)(C)C1=CC(=O)C(=O)C=C1</smiles>

Yield

GPC

$\mathrm{T}_{\mathrm{g}}$

TGA

${ }^{1} \mathrm{H}-\mathrm{NMR}(500 \mathrm{MHz})$

in $\mathrm{DMSO}_{6} \mathrm{~d}_{6}$

FT-IR $\left[\mathrm{cm}^{-1}\right]$

$59 \%$

$\mathrm{M}_{w, a p p}=11.4 \mathrm{~kg} / \mathrm{mol}, \mathrm{Ð}=2.0, \mathrm{DP}(\mathrm{AA}+\mathrm{BB})=27$

$123{ }^{\circ} \mathrm{C}$

$320{ }^{\circ} \mathrm{C}$ (maximal degradation rate)

$\delta[\mathrm{ppm}]$ 9. $52-8.29$ (m, br.; 4H; ArOH), 7.31 - 6.27 (m, br.; 8H; BQ and DT ArH), 4.23 - 3.50 (m, br.; 3H; DT aliphatic H), 1.71 - 1.16 (m, br.; 6H; BQ aliphatic H).

3368 (s), 2968 (m), 2926 (w), 2872 (w), 1699 (w), 1591 (m), 1508

(m), 1477 (s), 1411 (s), 1361 (m), 1338 (m), 1320 (m), 1268 (s), 1231

(s), 1198 (s), 1150 (s), 1100 (m), 1091 (m), 971 (w), 953 (m), 862

(m), $814(\mathrm{w}), 782(\mathrm{w}), 731(\mathrm{w}), 648(\mathrm{w})$.

MALDI-TOF-MS calculated $(\mathrm{m} / \mathrm{z}):[\mathrm{M}(4 \mathrm{AA}+4 \mathrm{BB})+\mathrm{Na}]^{+}=1729$

found $(\mathrm{m} / \mathrm{z}):[\mathrm{M}(4 \mathrm{AA}+4 \mathrm{BB})+\mathrm{Na}]^{+}=1729$
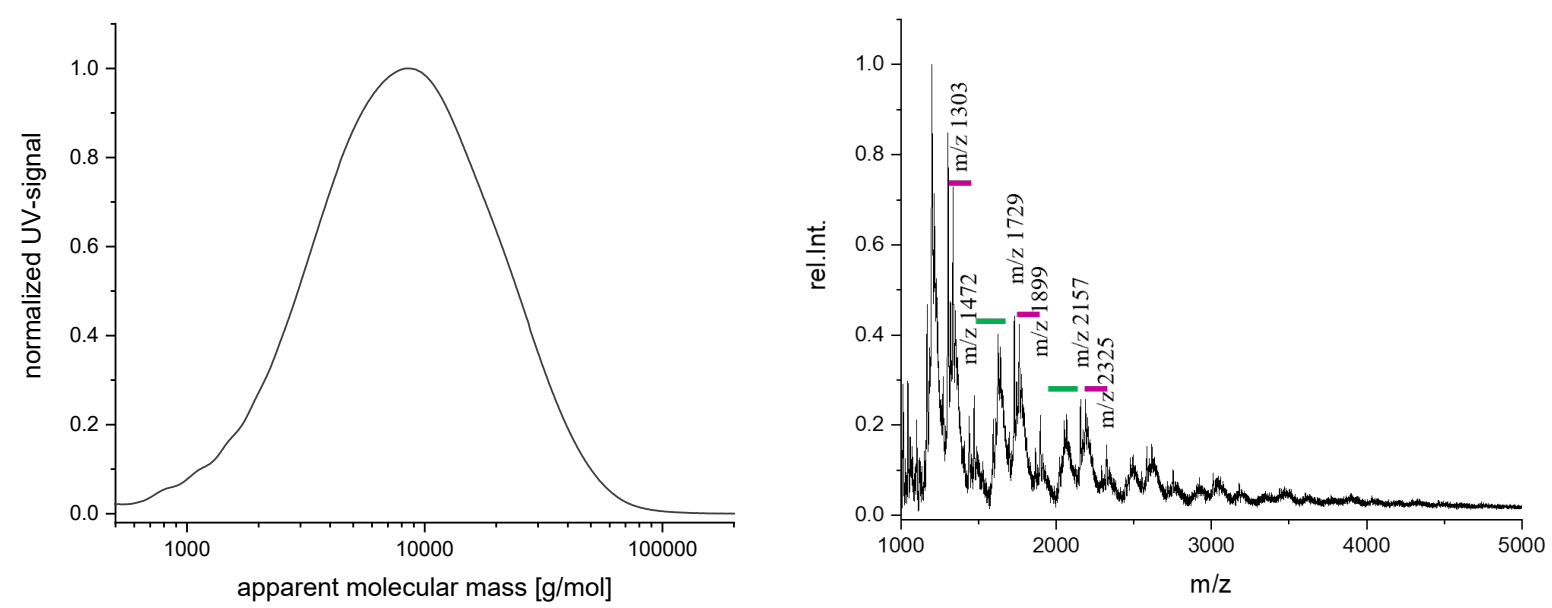

Figure S 18. p(BQA-BMT) analysis. GPC chromatogram at $280 \mathrm{~nm}$ (left) and MALDI-TOF-MS spectrum (right $\mathrm{m} / \mathrm{z}$ values were assigned to $\mathrm{Na}^{+}$ion adducts of the oligomer showing the homologous raw of $\mathrm{AA}$ and $\mathrm{BB}$ monomer additions in the TCC-polymer product. Colored bars represent the $\mathrm{m} / \mathrm{z}$ differences corresponding to the repeating units, where green refers to biscatechols and purple to dithiols. Despite optimization of matrix and sample preparation means, the TCC polymer shows a challenging desorption behavior, preventing to obtain well resolved MALDI-TOF-MS spectra). 
<smiles>CC(C)(C1=CC(=O)C(=O)C=C1)c1cc(O)c(O)c(SCC(O)C(O)CSc2cc(C(C)(C)C3=CC(=O)C(=O)C(=O)C3)cc(O)c2O)c1</smiles>

Yield

GPC

$\mathrm{T}_{\mathrm{g}}$

TGA

${ }^{1} \mathrm{H}-\mathrm{NMR}(500 \mathrm{MHz})$ in $\mathrm{DMSO}_{-} \mathrm{d}_{6}$

FT-IR $\left[\mathrm{cm}^{-1}\right]$

$72 \%$

$\mathrm{M}_{w, \text { app }}=2.21 \mathrm{~kg} / \mathrm{mol}, \mathrm{Ð}=1.2, \mathrm{DP}(\mathrm{AA}+\mathrm{BB})=5$

$124{ }^{\circ} \mathrm{C}$

$323{ }^{\circ} \mathrm{C}$ (maximal degradation rate)

$\delta[\mathrm{ppm}] 9.29-8.14$ (m, br.; 4H; BQ ArOH), $7.14-6.08$ (m, br.; 4H; BQ ArH), 5.36 - 4.76 (m, br.; 2H; aliphatic DT H), $3.86-2.214$ (m, br.; 9H; DT aliphatic $\mathrm{H}$; integral includes signal of $\mathrm{H}_{2} \mathrm{O}$ and DMSO), $1.94-1.19$ (m, br.; 6H; BQ aliphatic H).

3326 (s), 2970 (m), 2931 (m), 1698 (m), 1591 (m), 1479 (m), 1412 (m), 1360 (m), 1272 (mH), 1229 (s), 1203 (s), 1156 (m), 1088 (m), 1041 (m), 973 (w), 955 (m), $908(\mathrm{w}), 863$ (m), $816(\mathrm{w}), 785(\mathrm{w}), 732$ (w).

MALDI-TOF-MS $\quad$ calculated $(\mathrm{m} / \mathrm{z}):[\mathrm{M}(4 \mathrm{AA}+4 \mathrm{BB})+\mathrm{Na}]^{+}=1665.4$ found $(\mathrm{m} / \mathrm{z}):[\mathrm{M}(4 \mathrm{AA}+4 \mathrm{BB})+\mathrm{Na}]^{+}=1664.9$
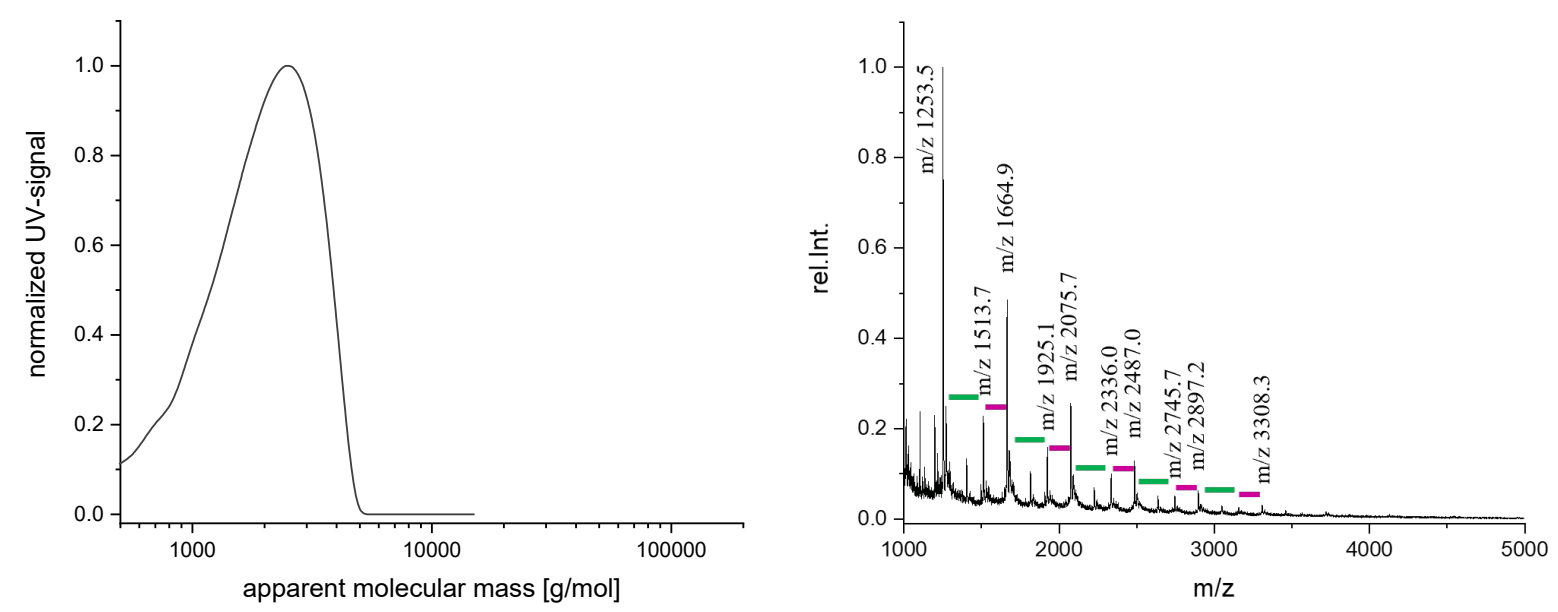

Figure S 19. p(BQA-DTT) analysis. GPC chromatogram at $280 \mathrm{~nm}$ (left) and MALDI-TOF-MS spectrum (right, $\mathrm{m} / \mathrm{z}$ values were assigned to $\mathrm{Na}+$ ion adducts of the oligomer showing the homologous raw of AA and $\mathrm{BB}$ monomer additions in the TCC-polymer product. Colored bars represent the $\mathrm{m} / \mathrm{z}$ differences corresponding to the repeating units, where green refers to biscatechols and purple to dithiols). 
<smiles></smiles>

Yield

GPC

$\mathrm{T}_{\mathrm{g}}$

TGA

${ }^{1} \mathrm{H}-\mathrm{NMR}(500 \mathrm{MHz})$ in $\mathrm{DMSO}_{-} \mathrm{d}_{6}$

FT-IR $\left[\mathrm{cm}^{-1}\right]$

$58 \%$

$\mathrm{M}_{w, a p p}=4.89 \mathrm{~kg} / \mathrm{mol}, \mathrm{Ð}=1.5, \mathrm{DP}(\mathrm{AA}+\mathrm{BB})=13$

$121{ }^{\circ} \mathrm{C}$

$315^{\circ} \mathrm{C}$ (maximal degradation rate)

$\delta[\mathrm{ppm}] 9.47$ - 8.01 (m, br.; 4H; BQ ArOH), 7.08 - 6.03 (m, br.; 4H; BQ ArH), 5.37 - 4.82 (m, br.; 1H; DT aliphatic H), $3.86-2.73$ (m, br.; $6 \mathrm{H}$; DT aliphatic $\mathrm{H}$; integral includes signal of $\mathrm{H}_{2} \mathrm{O}$ ), $1.93-1.12$ (m, br.; 6H; BQ aliphatic $\mathrm{H}$ ).

3356 (s), 2968 (m), 2935 (m), 2881 (w), 1749 (w), 1735 (w), 1698

(w), 1649 (w), 1478 (s), 1411 (m), 1363 (m), 1339 (m), 1271 (s), 1230

(s), 1202 (s), 1152 (s), 1091 (m), 1035 (w), $971(\mathrm{w}), 956$ (m), 908 (w), $864(\mathrm{~m}), 814(\mathrm{w}), 785(\mathrm{w}), 733(\mathrm{w}), 650(\mathrm{w})$.

MALDI-TOF-MS calculated (m/z): $[\mathrm{M}(4 \mathrm{AA}+4 \mathrm{BB})+\mathrm{Na}]^{+}=1545$

found $(\mathrm{m} / \mathrm{z}):[\mathrm{M}(4 \mathrm{AA}+4 \mathrm{BB})+\mathrm{Na}]^{+}=1545$
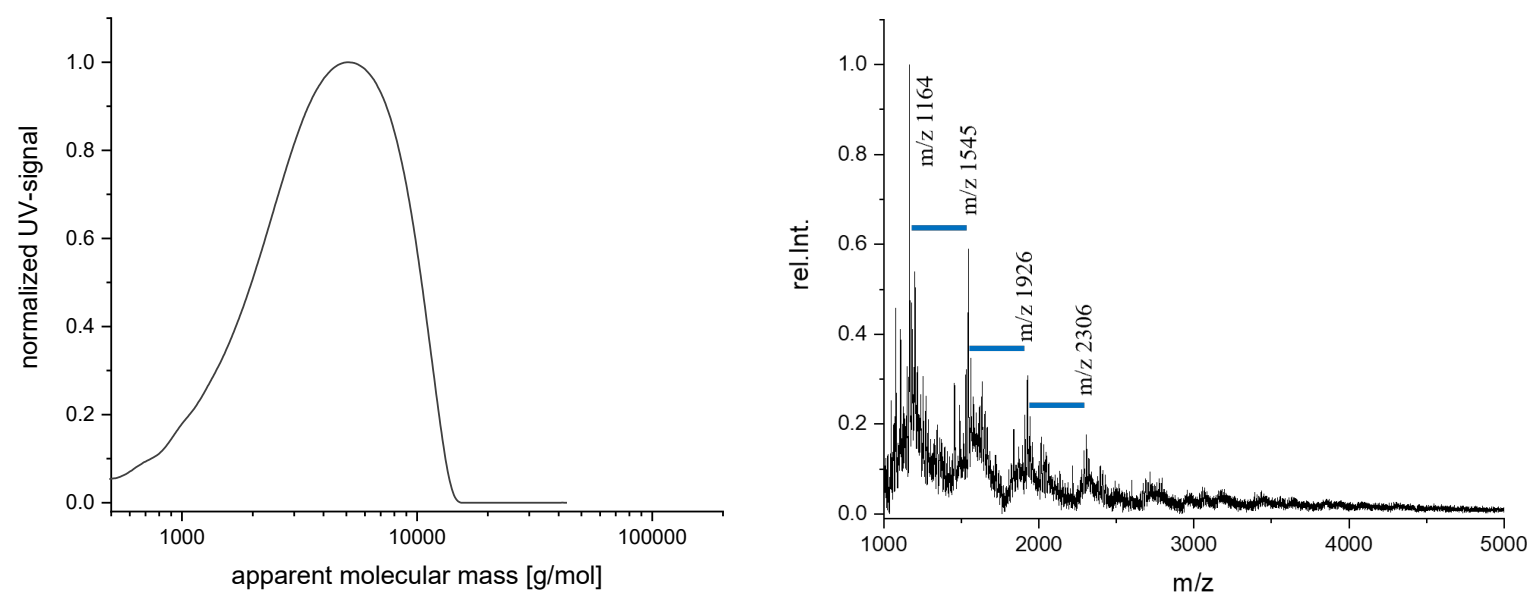

Figure S 20. p(BQA-DMP) analysis. GPC chromatogram at $280 \mathrm{~nm}$ (left) and MALDI-TOF-MS spectrum (right, $\mathrm{m} / \mathrm{z}$ values were assigned to $\mathrm{Na}+$ ion adducts of the oligomer showing the homologous raw of $\mathrm{AA}$ and $\mathrm{BB}$ monomer additions in the TCC-polymer product. Blue colored bars represent the difference corresponding to AABB dimer segments. Despite optimization of matrix and sample preparation means, the TCC polymer shows a challenging desorption behavior, preventing to obtain well resolved MALDI-TOF-MS spectra). 
<smiles>CC(C)(C)c1cc(O)c(O)c(SCC(=O)OCCOC(=O)CSc2cc(C(C)(C)C3=CC(=O)C(=O)C(=O)C3)cc(O)c2O)c1</smiles>

Yield

GPC

$\mathrm{T}_{\mathrm{g}}$

TGA

${ }^{1} \mathrm{H}-\mathrm{NMR}(500 \mathrm{MHz})$ in $\mathrm{DMSO}_{-} \mathrm{d}_{6}$

FT-IR $\left[\mathrm{cm}^{-1}\right]$

$55 \%$

$\mathrm{M}_{w, a p p}=6.48 \mathrm{~kg} / \mathrm{mol}, \mathrm{Ð}=1.8, \mathrm{DP}(\mathrm{AA}+\mathrm{BB})=14$

$72{ }^{\circ} \mathrm{C}$

$354{ }^{\circ} \mathrm{C}$ (maximal degradation rate)

$\delta[\mathrm{ppm}] 9.91$ - 8.39 (m, br.; 4H; BQ ArOH), 7.30 - 6.27 (m, br.; 4H; BQ ArH), 4.40 - 3.08 (m, br.; 4H; DT aliphatic H, integral includes signal of $\mathrm{H}_{2} \mathrm{O}$ ), 1,89 - 1.29 (m, br.; 6H; BQ aliphatic $\mathrm{H}$ ).

3383 (s), 2968 (m), 1733 (s), 1718 (s), 1701 (s), 1652 (w), 1590 (m), 1478 (s), 1412 (m), 1365 (m), 1341 (m), 1271 (s), 1230 (s), 1183 (s), 1155 (s), 1127 (s), 1054 (w), 971 (m), 960 (m), 866 (m), 817 (w), 785 (w), $733(\mathrm{w}), 650(\mathrm{w})$.

MALDI-TOF-MS calculated $(\mathrm{m} / \mathrm{z}):[\mathrm{M}(4 \mathrm{AA}+4 \mathrm{BB})+\mathrm{Na}]^{+}=1889.3$

found $(\mathrm{m} / \mathrm{z}):[\mathrm{M}(4 \mathrm{AA}+4 \mathrm{BB})+\mathrm{Na}]^{+}=1889.6$
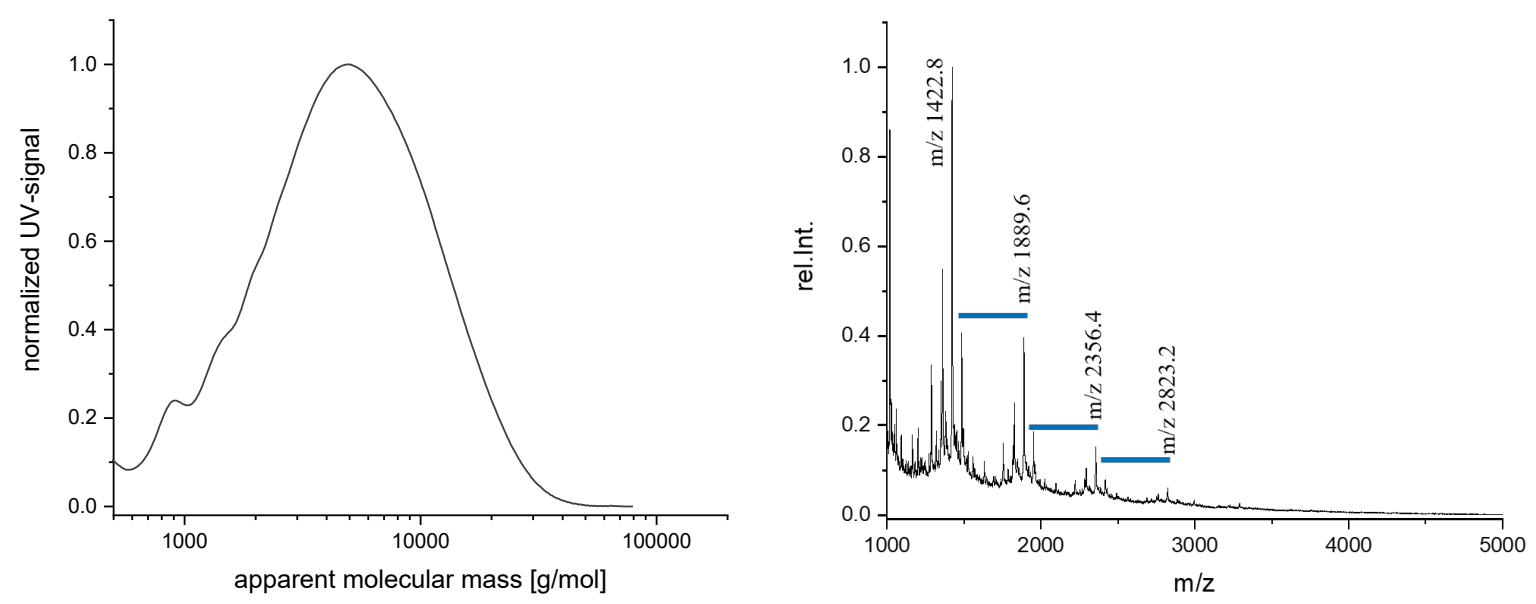

Figure S 21. p(BQA-GDMA) analysis. GPC chromatogram at $280 \mathrm{~nm}$ (left) and MALDI-TOF-MS spectrum (right, $\mathrm{m} / \mathrm{z}$ values were assigned to $\mathrm{Na}+$ ion adducts of the oligomer showing the homologous raw of AA and $\mathrm{BB}$ monomer additions in the TCC-polymer product. Blue colored bars represent the difference corresponding to AABB dimer segments). 
<smiles>CC(C)(C1=CC(=O)C(=O)C=C1)c1cc(O)c(O)c(SCCC(=O)OCCOC(=O)CCSc2cc(C(C)(C)C3=CC(=O)C(=O)C(=O)C3)cc(O)c2O)c1</smiles>

Yield

GPC

$\mathrm{T}_{\mathrm{g}}$

TGA

${ }^{1} \mathrm{H}-\mathrm{NMR}(500 \mathrm{MHz})$ in $\mathrm{DMSO}_{-} \mathrm{d}_{6}$

FT-IR $\left[\mathrm{cm}^{-1}\right]$

MALDI-TOF-MS

$49 \%$

$\mathrm{M}_{w, a p p}=9.39 \mathrm{~kg} / \mathrm{mol}, \mathrm{Ð}=1.7, \mathrm{DP}(\mathrm{AA}+\mathrm{BB})=19$

$68^{\circ} \mathrm{C}$

$339^{\circ} \mathrm{C}$ (maximal degradation rate)

$\delta[\mathrm{ppm}] 9.46-8.08$ (m, br.; 4H; BQ ArOH), 6,97- 6.22 (m, br.; 4H; BQ ArH), 4.31 - 4.05 (m, br.; 4H; DT aliphatic H), $3.11-2.04$ (m, br.; 10H; DT aliphatic H), $1.82-1.29$ (m, br.; 6H; BQ aliphatic H). 3378 (s), 297, (m), 1718 (s), 1591 (m), 1479 (m), 1410 (m), 1362 (m), 1340 (m), 1271 (m), 1230 (s), 1184 (s), 1134 (s), 1057 (m), 955 (m), $864(\mathrm{w}), 816(\mathrm{w}), 785(\mathrm{w}), 735(\mathrm{w}), 650(\mathrm{w})$.

calculated $(\mathrm{m} / \mathrm{z}):[\mathrm{M}(4 \mathrm{AA}+4 \mathrm{BB})+\mathrm{Na}]^{+}=2001.4$

found $(\mathrm{m} / \mathrm{z}):[\mathrm{M}(4 \mathrm{AA}+4 \mathrm{BB})+\mathrm{Na}]^{+}=2001.8$
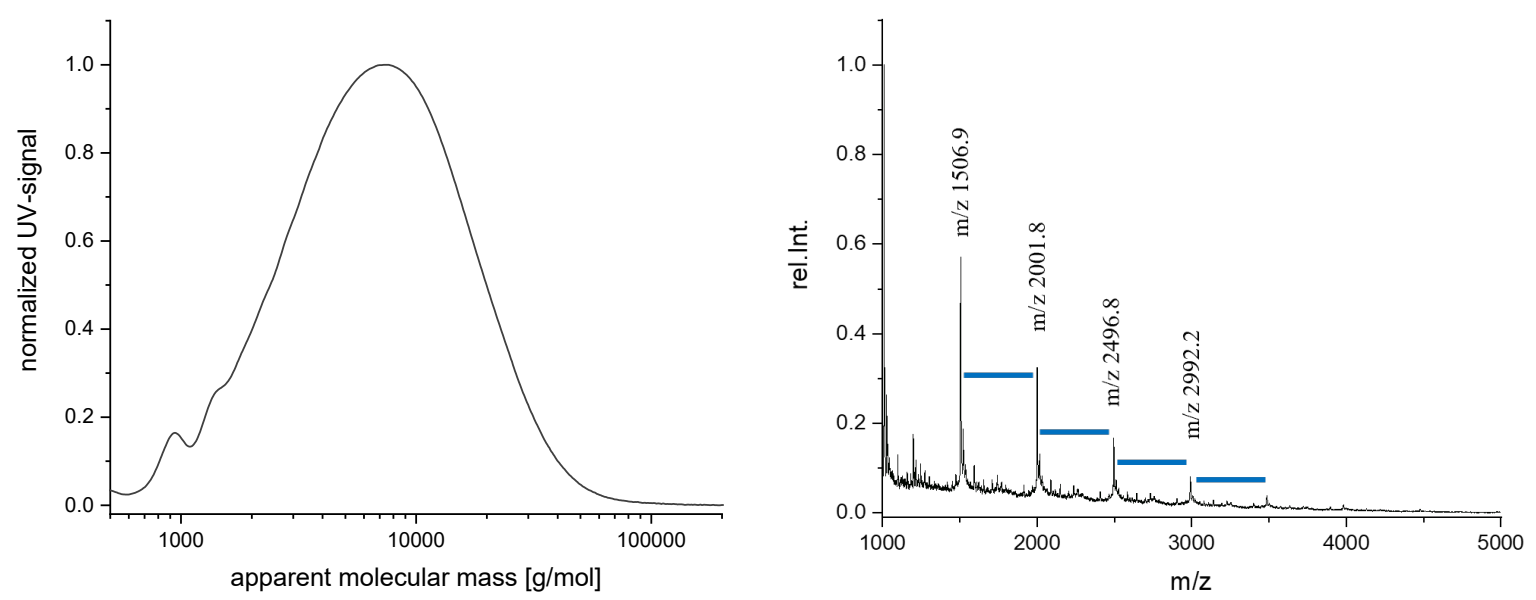

Figure S 22. p(BQA-GDMP) analysis. GPC chromatogram at $280 \mathrm{~nm}$ (left) and MALDI-TOF-MS spectrum (right, $\mathrm{m} / \mathrm{z}$ values were assigned to $\mathrm{Na}+$ ion adducts of the oligomer showing the homologous raw of $\mathrm{AA}$ and $\mathrm{BB}$ monomer additions in the TCC-polymer product. Blue colored bars represent the difference corresponding to AABB dimer segments). 
<smiles>CC(C)(C1=CC(=O)C(=O)C=C1)c1cc(O)c(O)c(SCCCCSc2cc(C(C)(C)C3=CC(=O)C(=O)C(=O)C3)cc(O)c2O)c1</smiles>

Yield

GPC

$\mathrm{T}_{\mathrm{g}}$

TGA

${ }^{1} \mathrm{H}-\mathrm{NMR}(500 \mathrm{MHz})$ in DMSO-d 6

FT-IR $\left[\mathrm{cm}^{-1}\right]$

$39 \%$

$\mathrm{M}_{w, a p p}=5.21 \mathrm{~kg} / \mathrm{mol}, \mathrm{Ð}=1.6, \mathrm{DP}(\mathrm{AA}+\mathrm{BB})=14$

$95^{\circ} \mathrm{C}$

$342{ }^{\circ} \mathrm{C}$ (maximal degradation rate)

$\delta[\mathrm{ppm}] 9.52$ - 7.83 (m, br.; 4H; BQ ArOH), 7.10 - 6.16 (m, br.; 4H; BQ ArH), $2.96-2.55$ (m, br.; 3H; DT aliphatic H), $2.36-2.00$ (m, br.; 1H; DT aliphatic H), 1.83 - 0.92 (m, br.; 12H; BQ and DT aliphatic $\mathrm{H})$.

3366 (s), 2966 (m), 2930 (m), 2870 (w), 1749 (w), 1741 (w), 1699 (m), 1591 (m), 1508 (w), 1477 (s), 1412 (s), 1361 (m), 1338 (m), 1319 (m), 1271 (s), 1230 (s), 1198 (s), 1149 (s), 1090 (m), 655 (m), $908(\mathrm{w}), 862(\mathrm{~m}), 816(\mathrm{w}), 785(\mathrm{w}), 733(\mathrm{w}), 650(\mathrm{w})$.

MALDI-TOF-MS calculated $(\mathrm{m} / \mathrm{z}):[\mathrm{M}(4 \mathrm{AA}+4 \mathrm{BB})+\mathrm{Na}]^{+}=1537$ found $(\mathrm{m} / \mathrm{z}):[\mathrm{M}(4 \mathrm{AA}+4 \mathrm{BB})+\mathrm{Na}]^{+}=1537$
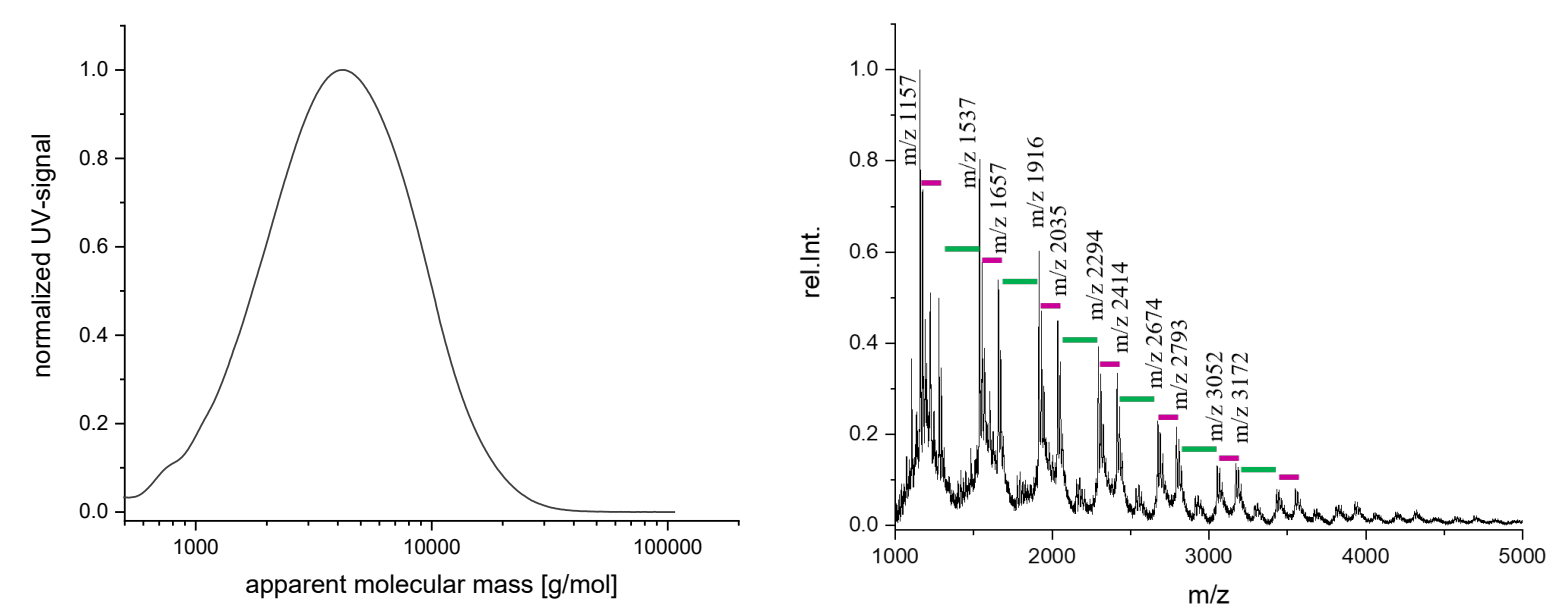

Figure S 23. p(BQA-BDT) analysis. GPC chromatogram at $280 \mathrm{~nm}$ (left) and MALDI-TOF-MS spectrum (right, $\mathrm{m} / \mathrm{z}$ values were assigned to $\mathrm{Na}+$ ion adducts of the oligomer showing the homologous raw of $\mathrm{AA}$ and $\mathrm{BB}$ monomer additions in the TCC-polymer product. Colored bars represent the $\mathrm{m} / \mathrm{z}$ differences corresponding to the repeating units, where green refers to biscatechols and purple to dithiols). 
<smiles>CC(C)(C1=CC(=O)C(=O)C=C1)c1cc(O)c(O)c(SCCCCCCSc2cc(C(C)(C)C3=CC(O)C(O)C=C3)cc(O)c2O)c1</smiles>

Yield

GPC

$\mathrm{T}_{\mathrm{g}}$

TGA

${ }^{1} \mathrm{H}-\mathrm{NMR}(500 \mathrm{MHz})$ in $\mathrm{DMSO}_{-} \mathrm{d}_{6}$

FT-IR $\left[\mathrm{cm}^{-1}\right]$

$47 \%$

$\mathrm{M}_{w, a p p}=7.18 \mathrm{~kg} / \mathrm{mol}, \mathrm{Ð}=1.7, \mathrm{DP}(\mathrm{AA}+\mathrm{BB})=18$

$72{ }^{\circ} \mathrm{C}$

$351{ }^{\circ} \mathrm{C}$ (maximal degradation rate)

$\delta[\mathrm{ppm}] 9.31$ - 7.88 (m, br.; 4H; BQ ArOH),6.97 - 6.22 (m, br.; 4H; BQ ArH), 2.93 - 1.98 (m, br.; 7H; DT aliphatic H; integral includes signal of DMSO), 1.86 - 0.88 (m, br.; 16H; BQ and DT aliphatic H). 3366 (s), 2964 (m), 2928 (s), 2856 (m), 1748 (w), 1736 (w), 1699 (w), 1649 (w), 1593 (m), 1508 (w), 1477 (s), 1412 (s), 1362 (m), 1338 (m), 1319 (m), 1267 (s), 1230 (s), 1198 (s), 1149 (s), 1090 (m), 955 (m), 908 (w), $864(\mathrm{~m}), 814(\mathrm{w}), 783(\mathrm{w}), 731(\mathrm{w}), 648(\mathrm{w})$.

MALDI-TOF-MS calculated $(\mathrm{m} / \mathrm{z}):[\mathrm{M}(4 \mathrm{AA}+4 \mathrm{BB})+\mathrm{Na}]^{+}=1650$ found $(\mathrm{m} / \mathrm{z}):[\mathrm{M}(4 \mathrm{AA}+4 \mathrm{BB})+\mathrm{Na}]^{+}=1650$
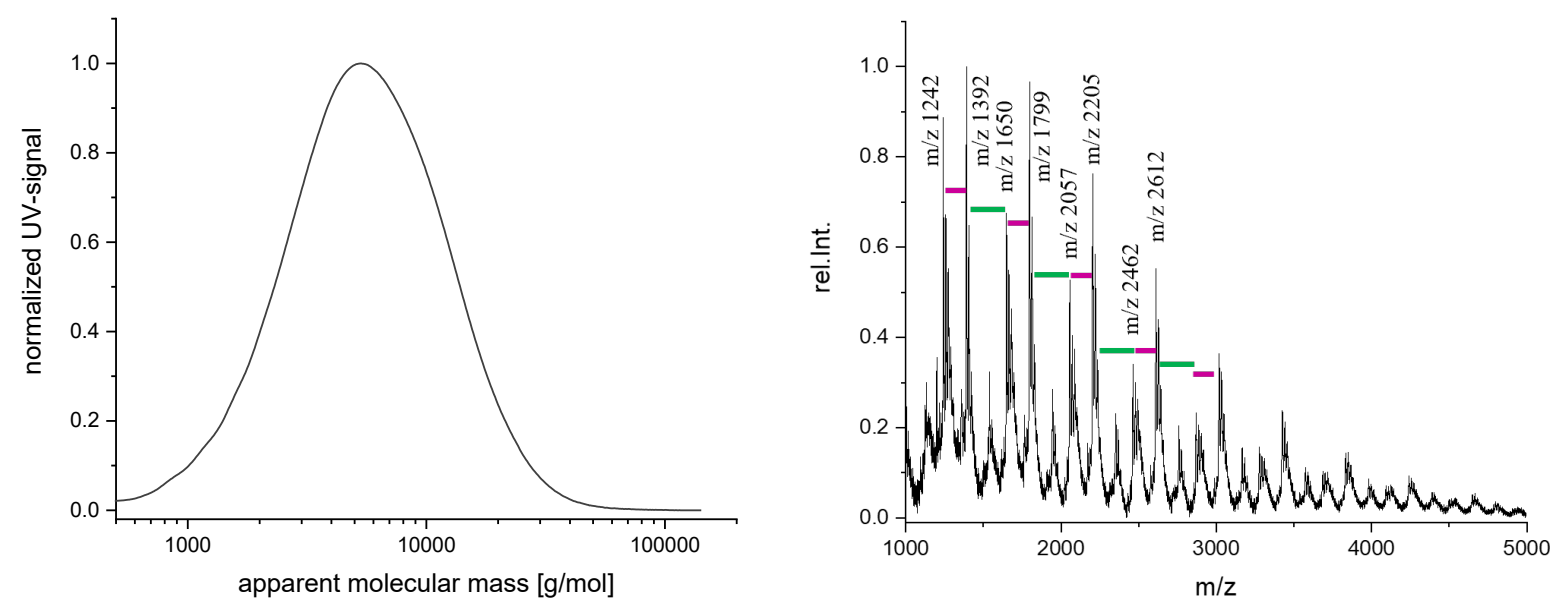

Figure S 24. p(BQA-HDT) analysis. GPC chromatogram at $280 \mathrm{~nm}$ (left) and MALDI-TOF-MS spectrum (right, $\mathrm{m} / \mathrm{z}$ values were assigned to $\mathrm{Na}+$ ion adducts of the oligomer showing the homologous raw of $\mathrm{AA}$ and $\mathrm{BB}$ monomer additions in the TCC-polymer product. Colored bars represent the $\mathrm{m} / \mathrm{z}$ differences corresponding to the repeating units, where green refers to biscatechols and purple to dithiols). 


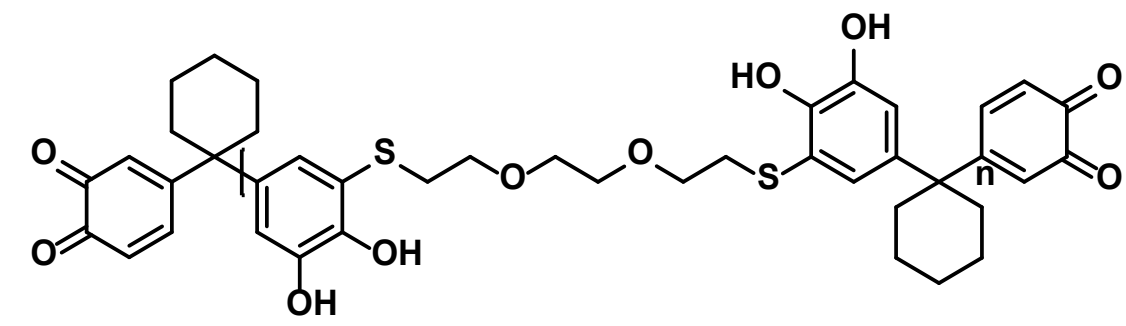

$73 \%$

Yield

GPC

$\mathrm{T}_{\mathrm{g}}$

TGA

${ }^{1} \mathrm{H}-\mathrm{NMR}(500 \mathrm{MHz})$ in $\mathrm{DMSO}_{-} \mathrm{d}_{6}$

FT-IR $\left[\mathrm{cm}^{-1}\right]$
$\mathrm{M}_{w, a p p}=9.06 \mathrm{~kg} / \mathrm{mol}, \mathrm{Ð}=2.1, \mathrm{DP}(\mathrm{AA}+\mathrm{BB})=19$

$83{ }^{\circ} \mathrm{C}$

$350{ }^{\circ} \mathrm{C}$ (maximal degradation rate)

$\delta[\mathrm{ppm}] 9.35$ - 8.01 (m, br.; 4H; BQ ArOH), 7.19 - 6.40 (m, br.; 4H; BQ ArH), 3.66 - 3.19 (m, br.; 9H; DT aliphatic H; integral includes signal of $\mathrm{H}_{2} \mathrm{O}$ ), $3.14-2.76$ (m, br.; 4H; DT aliphatic $\mathrm{H}$ ), $2.40-1.82$ (m, br.; 4H; BQ aliphatic H), 1.70 - 1.17 (m, br.; 6H; BQ aliphatic $\mathrm{H})$.

3325 (s), 2928 (s), 2858 (s), $1771(\mathrm{w}), 1749$ (w), 1733 (w), 1699 (m), 1653 (w), 1591 (m), 1541 (w), 1474 (s), 1410 (s), 1354(m), 1252 (s), 1217 (s), 1092 (s), 1040 (m), 965 (m), 900 (w), 854 (w), 808 (w), 733 (w), $685(\mathrm{w}), 653(\mathrm{w})$.

MALDI-TOF-MS calculated $(\mathrm{m} / \mathrm{z}):[\mathrm{M}(4 \mathrm{AA}+4 \mathrm{BB})+\mathrm{Na}]^{+}=1937.6$ found $(\mathrm{m} / \mathrm{z}):[\mathrm{M}(4 \mathrm{AA}+4 \mathrm{BB})+\mathrm{Na}]^{+}=1937.9$
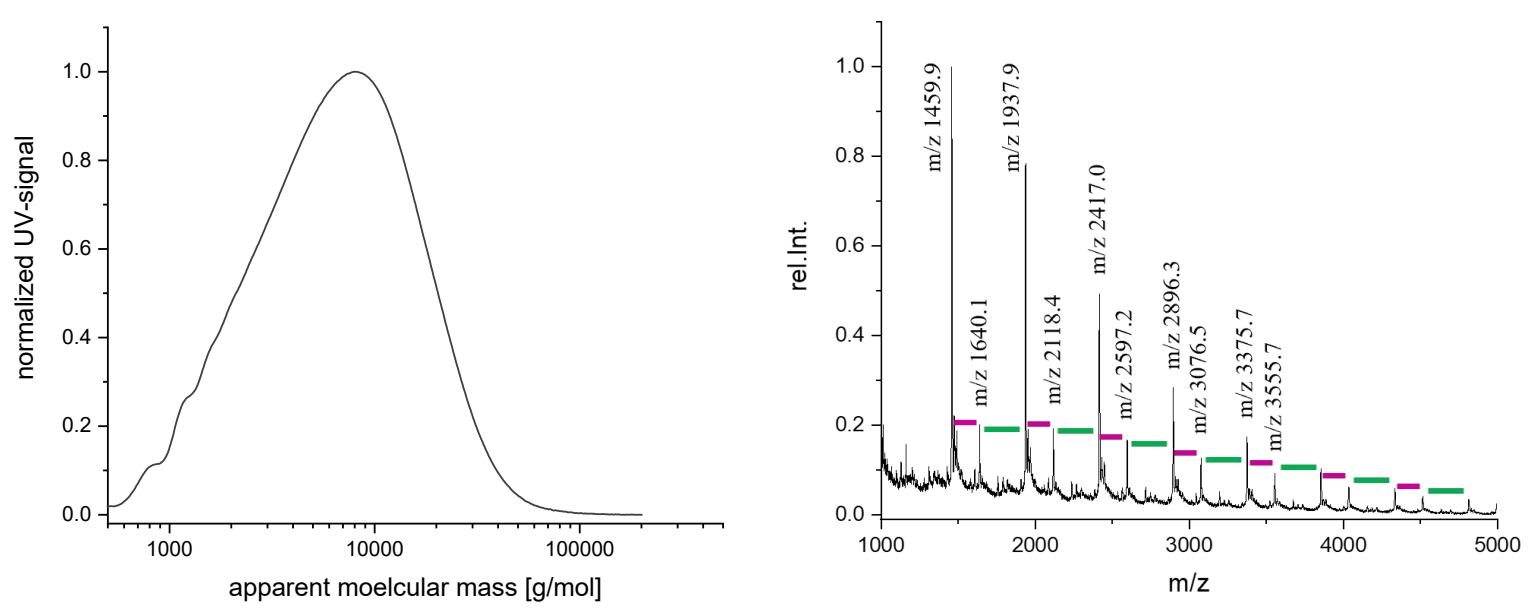

Figure S 25. p(BQZ-EDET) analysis. GPC chromatogram at $280 \mathrm{~nm}$ (left) and MALDI-TOF-MS spectrum (right, $\mathrm{m} / \mathrm{z}$ values were assigned to $\mathrm{Na}+$ ion adducts of the oligomer showing the homologous raw of AA and $\mathrm{BB}$ monomer additions in the TCC-polymer product. Colored bars represent the $\mathrm{m} / \mathrm{z}$ differences corresponding to the repeating units, where green refers to biscatechols and purple to dithiols). 
<smiles>O=C1C=CC(C2(c3cc(O)c(O)c(SCc4ccc(CSc5cc(C6(C7=CC(=O)C(=O)C=C7)CCCCC6)cc(O)c5O)cc4)c3)CCCCC2)=CC1=O</smiles>

Yield

GPC

$\mathrm{T}_{\mathrm{g}}$

TGA

${ }^{1} \mathrm{H}-\mathrm{NMR}(500 \mathrm{MHz})$ in $\mathrm{DMSO}_{6} \mathrm{~d}_{6}$

FT-IR $\left[\mathrm{cm}^{-1}\right]$

$80 \%$

$\mathrm{M}_{w, \text { app }}=14.7 \mathrm{~kg} / \mathrm{mol}, \mathrm{Ð}=2.7, \mathrm{DP}(\mathrm{AA}+\mathrm{BB})=31$

$126^{\circ} \mathrm{C}$

$341{ }^{\circ} \mathrm{C}$ (maximal degradation rate)

$\delta[\mathrm{ppm}] 9.47-8.10$ (m, br.; 4H; BQ ArOH), 7.33 - 6.22 (m, br.; 8H; BQ ArH), 4.27 - 3.71 (m, br.; 2H; DT aliphatic H), 3.25 - 2.96 (m, br.; 1H; DT aliphatic H), $2.48-1.69$ (m, br.; 4H; BQ aliphatic $\mathrm{H}$ ), $1.62-0.87$ (m, br.; 6H; BQ aliphatic H).

3375 (s), 2932 (s), 2856 (m), 1771 (w), 1749 (w), 1699 (m), 1653 (m), $1541(\mathrm{w}), 1508$ (m), 1475 (s), 1408 (s), 1354 (m), 1340 (m), 1317 (m), 1250 (s), 1217 (s), 1176 (m), 1142 (m), 1099 (w), 964 (w), $895(w), 852(w), 808(w), 728(w), 656(w)$.

MALDI-TOF-MS calculated $(\mathrm{m} / \mathrm{z}):[\mathrm{M}(4 \mathrm{AA}+4 \mathrm{BB})+\mathrm{Na}]^{+}=1890$ found $(\mathrm{m} / \mathrm{z}):[\mathrm{M}(4 \mathrm{AA}+4 \mathrm{BB})+\mathrm{Na}]^{+}=1890$
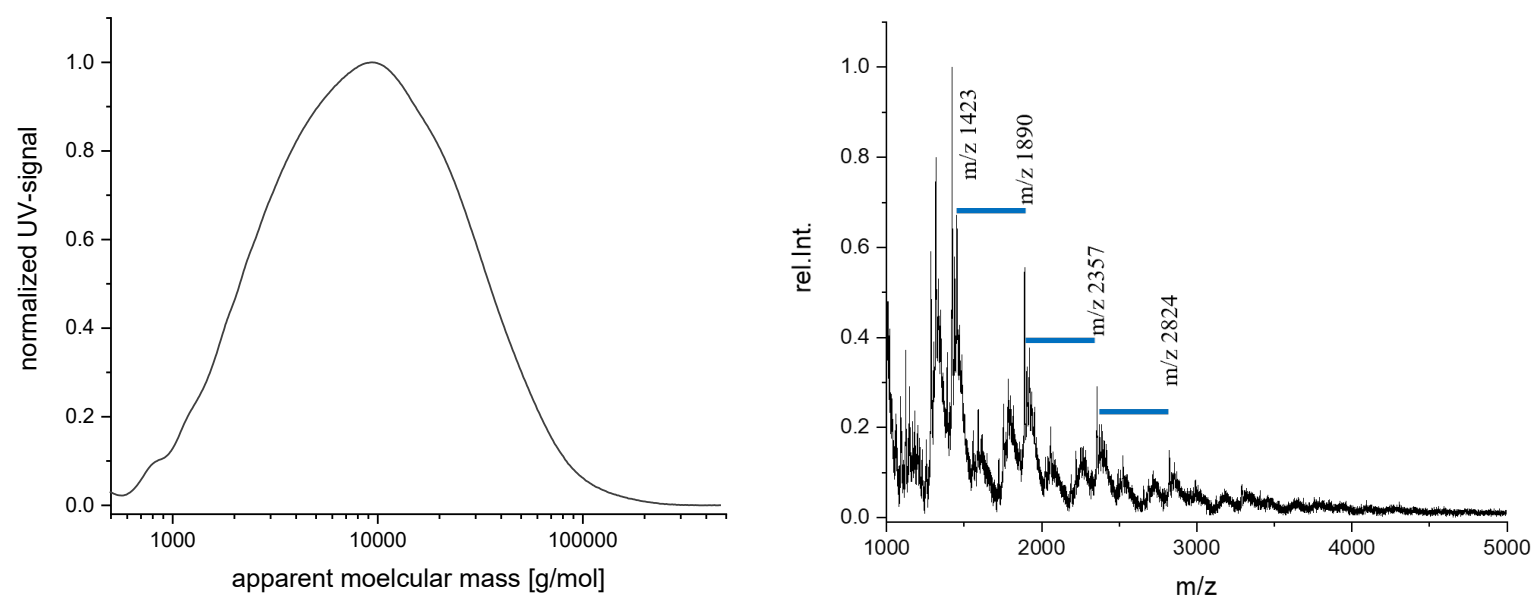

Figure S 26. p(BQZ-BMT) analysis. GPC chromatogram at $280 \mathrm{~nm}$ (left) and MALDI-TOF-MS spectrum (right, $\mathrm{m} / \mathrm{z}$ values were assigned to $\mathrm{Na}+$ ion adducts of the oligomer showing the homologous raw of $\mathrm{AA}$ and $\mathrm{BB}$ monomer additions in the TCC-polymer product. Blue colored bars represent the difference corresponding to AABB dimer segments. Despite optimization of matrix and sample preparation means, the TCC polymer shows a challenging desorption behavior, preventing to obtain well resolved MALDI-TOF-MS spectra). 
<smiles>O=C1C=CC(C2=CCC(=O)C=C2)(c2cc(O)c(O)c(SCC(O)C(O)CSc3cc(C4(C5=CC(=O)C(=O)C(=O)C5)CCCCC4)cc(O)c3O)c2)C=C1</smiles>

$76 \%$

Yield

GPC

$\mathrm{T}_{\mathrm{g}}$

TGA

${ }^{1} \mathrm{H}-\mathrm{NMR}(500 \mathrm{MHz})$ in $\mathrm{DMSO}_{-} \mathrm{d}_{6}$

FT-IR $\left[\mathrm{cm}^{-1}\right]$
$\mathrm{M}_{w, \text { app }}=3.01 \mathrm{~kg} / \mathrm{mol}, \mathrm{Ð}=1.5, \mathrm{DP}(\mathrm{AA}+\mathrm{BB})=7$

$129^{\circ} \mathrm{C}$

$341{ }^{\circ} \mathrm{C}$ (maximal degradation rate)

$\delta[\mathrm{ppm}] 9.19$ - 8.11 (m, br.; 4H; BQ ArOH), 7.26 - 6.18 (m, br.; 4H; BQ ArH), 5.29 - 4.75 (m, br.; 1H; DT aliphatic H), $3.86-3.17$ (m, br.; 5H; DT aliphatic $\mathrm{H}$; integral includes signal of $\mathrm{H} 2 \mathrm{O}$ ), $3.09-2.53$ (m, br.; 3H; DT aliphatic H), 2.45 - 1.78 (m, br.; 10H; BQ aliphatic H), 1.66 - 1. 02 ( m, br.; 6H; BQ aliphatic $\mathrm{H}$ ).

3336 (s), 2930 (s), 2856 (m), 1771 (w), 1749 (w), 1699 (m), 1653 (w), 1593 (m), 1558 (w), 1475 (m), 1410 (m), 1364 (m), 1247 (s), 1232 (s), 1216 (s), 1090 (m), 1041 (m), 699 (m), 897 (w), 854 (w), $808(\mathrm{w}), 731(\mathrm{w}), 685(\mathrm{w}), 656(\mathrm{w})$.

MALDI-TOF-MS calculated $(\mathrm{m} / \mathrm{z}):[\mathrm{M}(4 \mathrm{AA}+4 \mathrm{BB})+\mathrm{Na}]^{+}=1825.5$ found $(\mathrm{m} / \mathrm{z}):[\mathrm{M}(4 \mathrm{AA}+4 \mathrm{BB})+\mathrm{Na}]^{+}=1825.8$
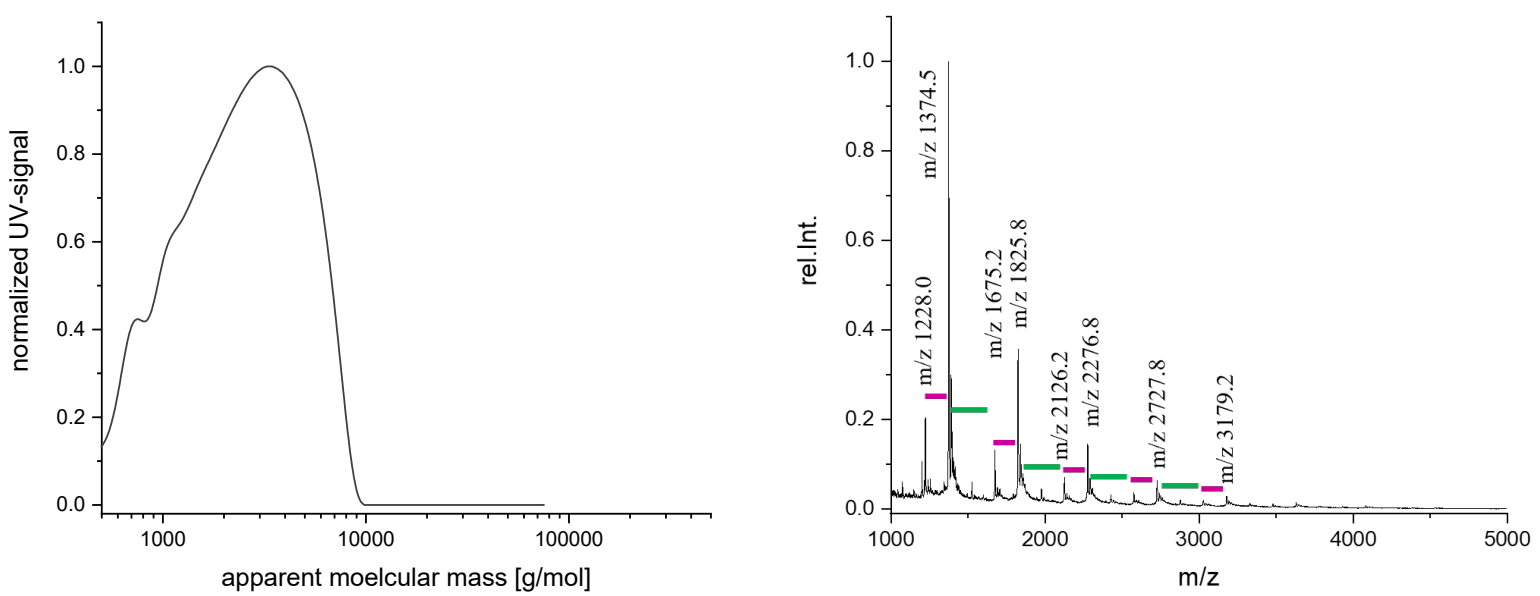

Figure S 27. p(BQZ-DTT) analysis. GPC chromatogram at $280 \mathrm{~nm}$ (left) and MALDI-TOF-MS spectrum (right, $\mathrm{m} / \mathrm{z}$ values were assigned to $\mathrm{Na}+$ ion adducts of the oligomer showing the homologous raw of $\mathrm{AA}$ and $\mathrm{BB}$ monomer additions in the TCC-polymer product. Colored bars represent the $\mathrm{m} / \mathrm{z}$ differences corresponding to the repeating units, where green refers to biscatechols and purple to dithiols). 


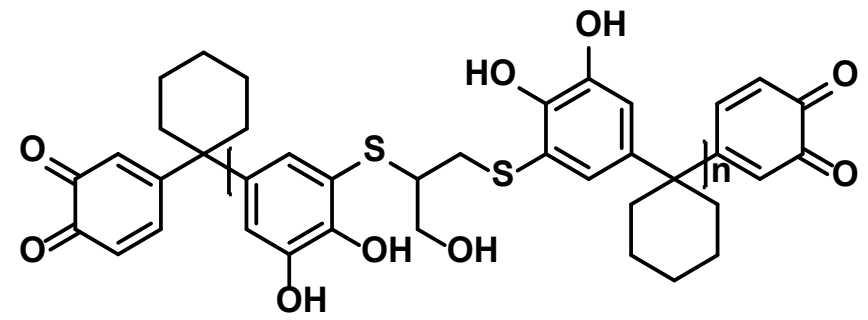

$58 \%$

Yield

GPC

$\mathrm{T}_{\mathrm{g}}$

TGA

${ }^{1} \mathrm{H}-\mathrm{NMR}(500 \mathrm{MHz})$ in $\mathrm{DMSO}_{6}$

FT-IR $\left[\mathrm{cm}^{-1}\right]$
$\mathrm{M}_{w, a p p}=5.65 \mathrm{~kg} / \mathrm{mol}, \mathrm{Ð}=1.8, \mathrm{DP}(\mathrm{AA}+\mathrm{BB})=13$

$113{ }^{\circ} \mathrm{C}$

$327^{\circ} \mathrm{C}$ (maximal degradation rate)

$\delta[\mathrm{ppm}] 9.42-8.08$ (m, br.; 4H; BQ ArOH), 7.21 - 6.26 (m, br.; 4H; BQ ArH), $5.41-4.88$ (m, br.; 1H; DT aliphatic H), $3.81-2.54$ (m, br.; 10H; DT aliphatic $\mathrm{H}$; integral includes signal of $\mathrm{H}_{2} \mathrm{O}$ ), 2. 43 1.78 (m, br.; 5H; BQ aliphatic H), $1.62-1.08$ (m, br.; 6H; BQ aliphatic $\mathrm{H})$.

3348 (s), 2934 (s), 2856 (m), 1772 (w), 1757 (w), 1781 (w), 1699 (w), 1653 (w), 1591 (w), 1541 (w), 1507 (w), 1474 (m), 1408 (m), 1364 (m), 1248 (s), 1217 (s), 1176 (m), 1147 (m), 1063 (w), 1038 (w), 965 (w), $895(w), 854(w), 808(w), 729(w), 685(w), 653(w)$.

MALDI-TOF-MS calculated $(\mathrm{m} / \mathrm{z}):[\mathrm{M}(4 \mathrm{AA}+4 \mathrm{BB})+\mathrm{Na}]^{+}=1705$ found $(\mathrm{m} / \mathrm{z}):[\mathrm{M}(4 \mathrm{AA}+4 \mathrm{BB})+\mathrm{Na}]^{+}=1706$
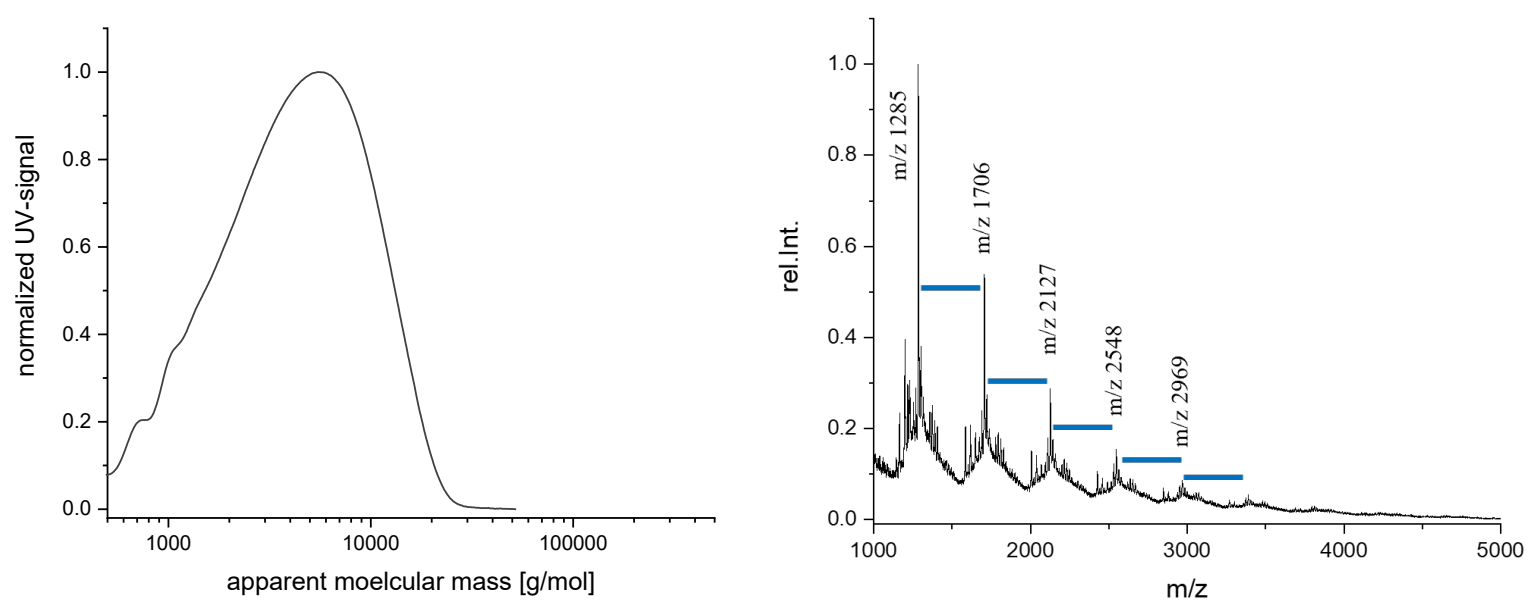

Figure S 28. p(BQZ-DMP) analysis. GPC chromatogram at $280 \mathrm{~nm}$ (left) and MALDI-TOF-MS spectrum (right, $\mathrm{m} / \mathrm{z}$ values were assigned to $\mathrm{Na}+$ ion adducts of the oligomer showing the homologous raw of $\mathrm{AA}$ and $\mathrm{BB}$ monomer additions in the TCC-polymer product. Blue colored bars represent the difference corresponding to AABB dimer segments). 
<smiles>O=C1C=CC(C2=CCC(=O)C=C2)(c2cc(O)c(O)c(SCC(=O)OCCOC(=O)CSc3cc(C4(C5=CC(=O)C(=O)C(=O)C5)CCCCC4)cc(O)c3O)c2)C=C1</smiles>

Yield

$46 \%$

GPC

$\mathrm{M}_{w, a p p}=6.70 \mathrm{~kg} / \mathrm{mol}, \mathrm{Ð}=2.0, \mathrm{DP}(\mathrm{AA}+\mathrm{BB})=13$

$\mathrm{T}_{\mathrm{g}}$

$96{ }^{\circ} \mathrm{C}$

TGA

$376^{\circ} \mathrm{C}$ (maximal degradation rate)

${ }^{1} \mathrm{H}-\mathrm{NMR}(500 \mathrm{MHz}) \quad \delta[\mathrm{ppm}] 9.88$ - 8.25 (m, br.; 3H; BQ ArOH), 7.32 - 6.22 (m, br.; 4H; in $\mathrm{DMSO}-\mathrm{d}_{6}$

BQ ArH), $4.36-3.42$ (m, br.; 6H; DT aliphatic H), $3.10-2.80$ (m, br.; 1H; DT aliphatic H), 2.48 - 1.87 (m, br.; 4H; BQ aliphatic $\mathrm{H}$ ), $1.67-1.13$ (m, br.; 6H; BQ aliphatic $\mathrm{H})$.

FT-IR $\left[\mathrm{cm}^{-1}\right]$

3373 (s), 2937 (s(, 2858 (m), 1734 (s), 1718 (s), 1593 (w), 1475 (m), $110(\mathrm{~m}), 1365$ (m), 1250 (s), 1175 (s), 1122 (s), $1055(\mathrm{w}), 699(\mathrm{w})$, $891(\mathrm{w}), 856(\mathrm{w}), 810(\mathrm{w}), 731(\mathrm{w}), 685(\mathrm{w}), 652(\mathrm{w})$.

MALDI-TOF-MS calculated $(\mathrm{m} / \mathrm{z}):[\mathrm{M}(4 \mathrm{AA}+4 \mathrm{BB})+\mathrm{Na}]^{+}=2049.4$

found $(\mathrm{m} / \mathrm{z}):[\mathrm{M}(4 \mathrm{AA}+4 \mathrm{BB})+\mathrm{Na}]^{+}=2050.4$
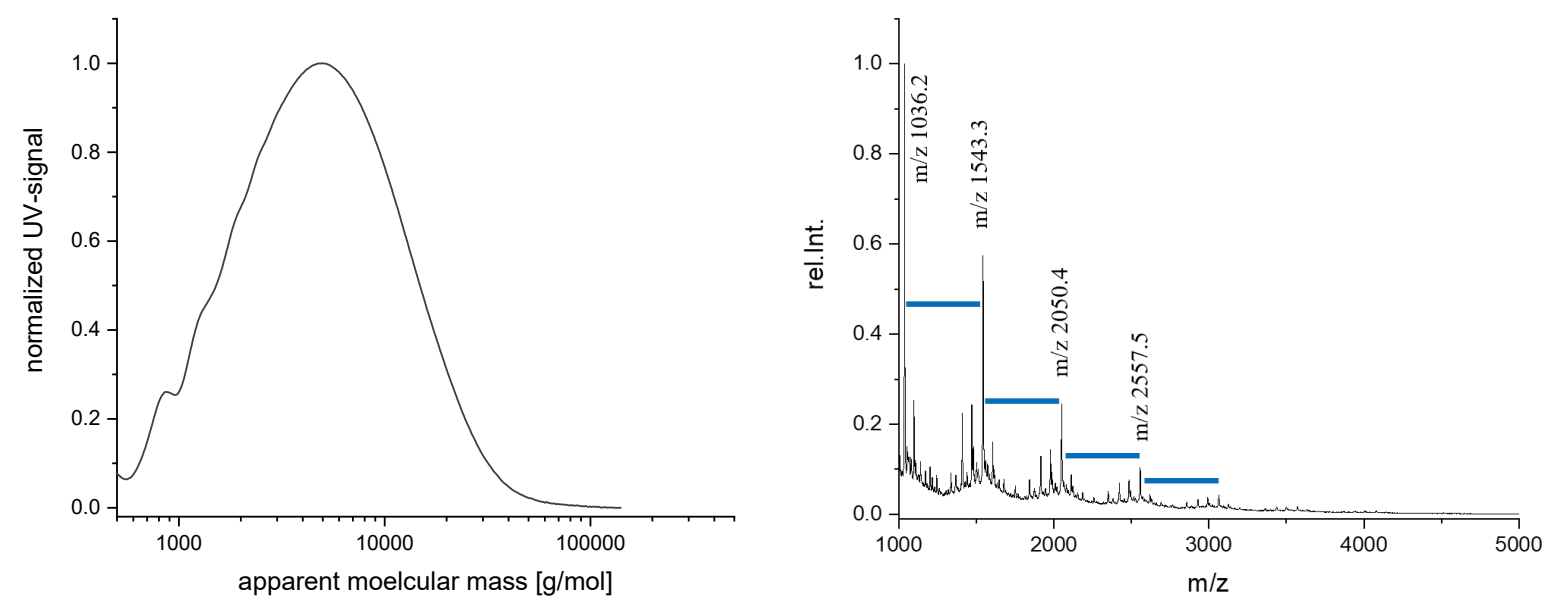

Figure S 29. p(BQZ-GDMA) analysis. GPC chromatogram at $280 \mathrm{~nm}$ (left) and MALDI-TOF-MS spectrum (right, $\mathrm{m} / \mathrm{z}$ values were assigned to $\mathrm{Na}+$ ion adducts of the oligomer showing the homologous raw of $\mathrm{AA}$ and $\mathrm{BB}$ monomer additions in the TCC-polymer product. Blue colored bars represent the difference corresponding to AABB dimer segments). 
<smiles>O=C(CCSc1cc(C2(C3=CC(=O)C(=O)C=C3)CCCCC2)cc(O)c1O)OCCOC(=O)CCSc1cc(C2(C3=CC(=O)C(=O)C(=O)C3)CCCCC2)cc(O)c1O</smiles>

Yield

$79 \%$

GPC

$\mathrm{M}_{w, a p p}=7.56 \mathrm{~kg} / \mathrm{mol}, \mathrm{Ð}=1.7, \mathrm{DP}(\mathrm{AA}+\mathrm{BB})=14$

$\mathrm{T}_{\mathrm{g}}$

$82{ }^{\circ} \mathrm{C}$

TGA

$347^{\circ} \mathrm{C}$ (maximal degradation rate)

${ }^{1} \mathrm{H}-\mathrm{NMR}(500 \mathrm{MHz}) \quad \delta[\mathrm{ppm}] 9.36-8.01$ (m, br.; 4H; BQ ArOH), 7.20 - 6.33 (m, br.; 4H; in $\mathrm{DMSO}_{-} \mathrm{d}_{6}$

BQ ArH), $4.39-4.04$ (m, br.; 4H; DT aliphatic H), $3.20-2.84$ (m, br.; 2H; DT aliphatic H), 2.58 - 1.89 (m, br.; 7H; DT aliphatic H; integral includes signal of DMSO), 1.68 - 1.22 (m, br.; $6 \mathrm{H}$; BQ aliphatic $\mathrm{H})$.

FT-IR $\left[\mathrm{cm}^{-1}\right]$ 3366 (s), 2939 (s), $2858(\mathrm{w}), 1734$ (s), $1650(\mathrm{w}), 1594(\mathrm{w}), 1541(\mathrm{w})$, $1520(\mathrm{w}), 1509$ (m), 1456 (m), 1408 (m), 1364 (m), 1248 (s), 1231 (s), $1216(\mathrm{~s}), 1176(\mathrm{~s}), 1136(\mathrm{~s}), 1054(\mathrm{w}), 966(\mathrm{w}), 931(\mathrm{w}), 895(\mathrm{w})$, $854(\mathrm{w}), 810(\mathrm{w}), 732(\mathrm{w}), 655(\mathrm{w})$.

MALDI-TOF-MS calculated $(\mathrm{m} / \mathrm{z}):[\mathrm{M}(4 \mathrm{AA}+4 \mathrm{BB})+\mathrm{Na}]^{+}=2161.6$ found $(\mathrm{m} / \mathrm{z}):[\mathrm{M}(4 \mathrm{AA}+4 \mathrm{BB})+\mathrm{Na}]^{+}=2162.7$
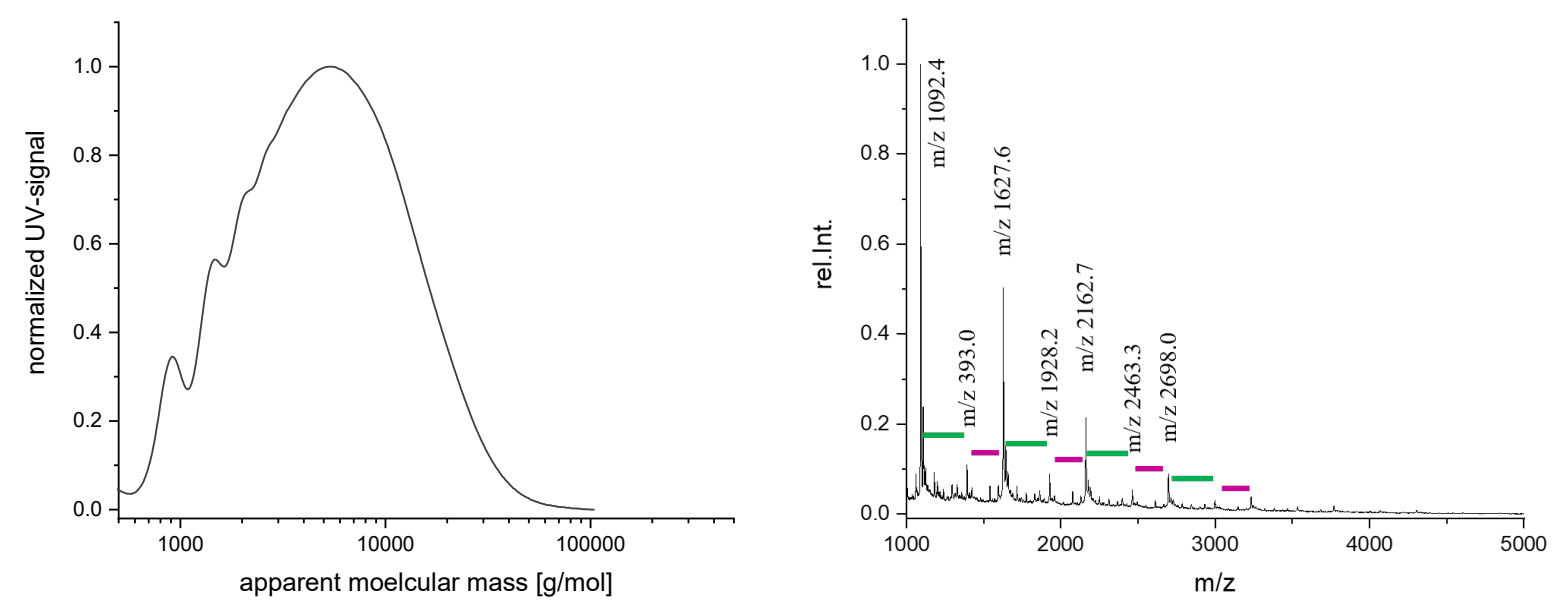

Figure S 30. p(BQZ-GDMP) analysis. GPC chromatogram at $280 \mathrm{~nm}$ (left) and MALDI-TOF-MS spectrum (right, $\mathrm{m} / \mathrm{z}$ values were assigned to $\mathrm{Na}+$ ion adducts of the oligomer showing the homologous raw of $\mathrm{AA}$ and $\mathrm{BB}$ monomer additions in the TCC-polymer product. Colored bars represent the $\mathrm{m} / \mathrm{z}$ differences corresponding to the repeating units, where green refers to biscatechols and purple to dithiols). 
<smiles>O=C1C=CC(C2=CCC(=O)C=C2)(c2cc(O)c(O)c(SCCCCSc3cc(C4(C5=CC(=O)C(=O)C(=O)C5)CCCCC4)cc(O)c3O)c2)C=C1</smiles>

Yield

GPC

$\mathrm{T}_{\mathrm{g}}$

TGA

${ }^{1} \mathrm{H}-\mathrm{NMR}(500 \mathrm{MHz})$ in $\mathrm{DMSO}_{-} \mathrm{d}_{6}$

FT-IR $\left[\mathrm{cm}^{-1}\right]$

$69 \%$

$\mathrm{M}_{w, a p p}=5.23 \mathrm{~kg} / \mathrm{mol}, \mathrm{Ð}=1.8, \mathrm{DP}(\mathrm{AA}+\mathrm{BB})=13$

$108^{\circ} \mathrm{C}$

$345^{\circ} \mathrm{C}$ (maximal degradation rate)

$\delta[\mathrm{ppm}] 9.27$ - 7.76 (m, br.; 4H; BQ ArOH), 7.26 - 6.13 (m, br.; 4H; BQ ArH), $3.05-2.39$ (m, br.; 5H; DT aliphatic H; integral includes signal of DMSO), 2.38 - 1.88 (m, br.; 1H; DT aliphatic H), 1.86 0.69 (m, br.; 12H; BQ and DT aliphatic H)

3364 (m), 2930 (s), 2856 (m), 1749 (m), 1699 (m), 1593 (m), 1508 (m), 1475 (s), 1410 (s), 1364 (s), 1248 (s), 1217 (s), 1176 (s), 1141 (m), $966(\mathrm{w}), 899(\mathrm{w}), 854(\mathrm{w}), 808(\mathrm{w}), 731(\mathrm{w}), 653(\mathrm{w})$.

MALDI-TOF-MS calculated $(\mathrm{m} / \mathrm{z}):[\mathrm{M}(4 \mathrm{AA}+4 \mathrm{BB})+\mathrm{Na}]^{+}=1698$ found $(\mathrm{m} / \mathrm{z}):[\mathrm{M}(4 \mathrm{AA}+4 \mathrm{BB})+\mathrm{Na}]^{+}=1697$
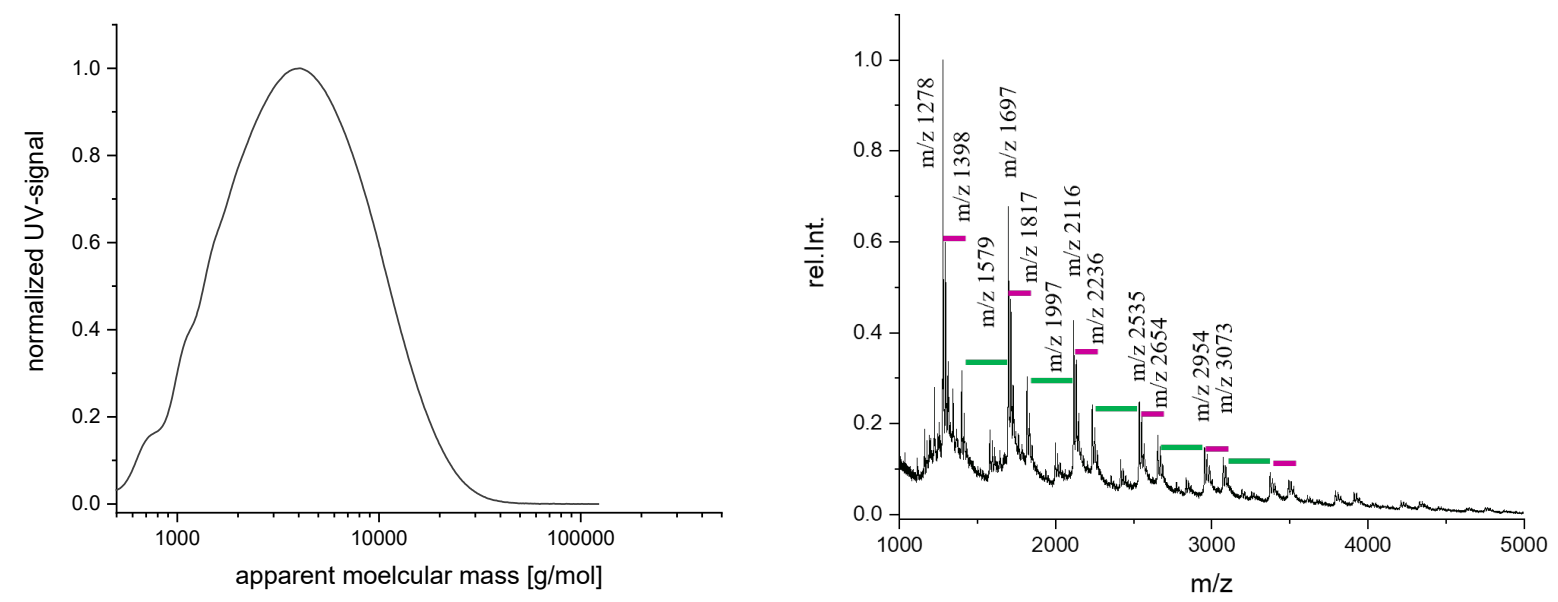

Figure S 31. p(BQZ-BDT) analysis. GPC chromatogram at $280 \mathrm{~nm}$ (left) and MALDI-TOF-MS spectrum (right, $\mathrm{m} / \mathrm{z}$ values were assigned to $\mathrm{Na}+$ ion adducts of the oligomer showing the homologous raw of AA and $\mathrm{BB}$ monomer additions in the TCC-polymer product. Colored bars represent the $\mathrm{m} / \mathrm{z}$ differences corresponding to the repeating units, where green refers to biscatechols and purple to dithiols). 
<smiles>O=C1C=CC(C2(c3cc(O)c(O)c(SCCCCCCSc4cc(C5(C6=CC(O)C(O)C=C6)CCCCC5)cc(O)c4O)c3)C=CC(=O)C(O)=C2)=CC1</smiles>

$70 \%$

Yield

GPC

$\mathrm{T}_{\mathrm{g}}$

TGA

${ }^{1} \mathrm{H}-\mathrm{NMR}(500 \mathrm{MHz})$ in $\mathrm{DMSO}_{-} \mathrm{d}_{6}$

FT-IR $\left[\mathrm{cm}^{-1}\right]$
$\mathrm{M}_{w, a p p}=9.31 \mathrm{~kg} / \mathrm{mol}, \mathrm{Ð}=2.1, \mathrm{DP}(\mathrm{AA}+\mathrm{BB})=21$

$99^{\circ} \mathrm{C}$

$351{ }^{\circ} \mathrm{C}$ (maximal degradation rate)

$\delta[\mathrm{ppm}] 9.25-7.80$ (m, br.; 4H; BQ ArOH), 7.25 - 6.25 (m, br.; 4H; BQ ArH), 2.99 - 2. 28 (m, br.; 6H; DT aliphatic H; integral includes signal of DMSO), 2.24 - 1.68 (m, br.; 4H; BQ aliphatic H), 1.68 0.80 (m, br.; 16H; BQ and DT aliphatic H)

3375 (m), 2928 (s), 2856 (m), 1749 (m), 1716 (m), 1699 (m), 1649 (w), 1595 (w), 1509 (w), 147a (m), 1457 (m), 1410 (m), 1364 (m), 1248 (s), 1231 (s), 1218 (s), 1176 (m), 1142 (m), 966 (w), 899 (w), $854(\mathrm{w}), 808(\mathrm{w}), 730(\mathrm{w}), 652(\mathrm{w})$.

MALDI-TOF-MS calculated $(\mathrm{m} / \mathrm{z}):[\mathrm{M}(4 \mathrm{AA}+4 \mathrm{BB})+\mathrm{Na}]^{+}=1810$ found $(\mathrm{m} / \mathrm{z}):[\mathrm{M}(4 \mathrm{AA}+4 \mathrm{BB})+\mathrm{Na}]^{+}=1810$
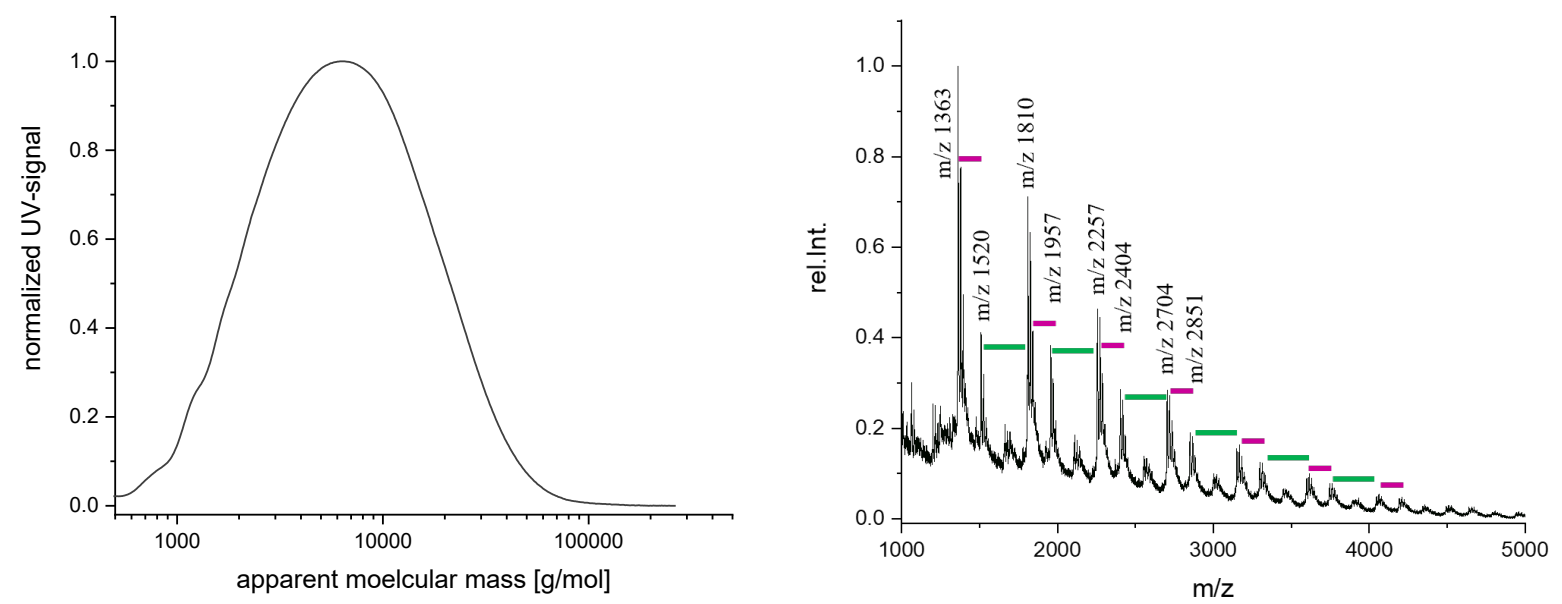

Figure S 32. p(BQZ-HDT) analysis. GPC chromatogram at $280 \mathrm{~nm}$ (left) and MALDI-TOF-MS spectrum (right, $\mathrm{m} / \mathrm{z}$ values were assigned to $\mathrm{Na}+$ ion adducts of the oligomer showing the homologous raw of $\mathrm{AA}$ and $\mathrm{BB}$ monomer additions in the TCC-polymer product. Colored bars represent the $\mathrm{m} / \mathrm{z}$ differences corresponding to the repeating units, where green refers to biscatechols and purple to dithiols). 


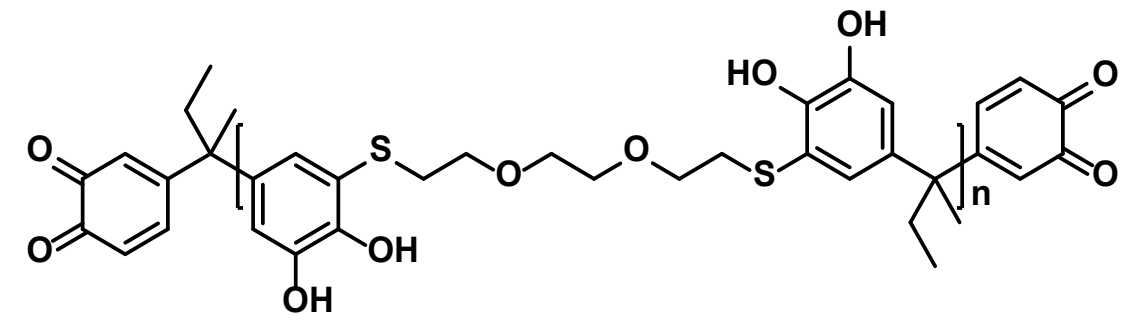

Yield

GPC

$\mathrm{T}_{\mathrm{g}}$

TGA

${ }^{1} \mathrm{H}-\mathrm{NMR}(500 \mathrm{MHz})$ in $\mathrm{DMSO}_{-} \mathrm{d}_{6}$

FT-IR $\left[\mathrm{cm}^{-1}\right]$

$49 \%$

$\mathrm{M}_{w, a p p}=10.8 \mathrm{~kg} / \mathrm{mol}, \mathrm{Ð}=1.7, \mathrm{DP}(\mathrm{AA}+\mathrm{BB})=24$

$69^{\circ} \mathrm{C}$

$346^{\circ} \mathrm{C}$ (maximal degradation rate)

$\delta[\mathrm{ppm}] 9.36-8.03$ (m, br.; 4H; BQ ArOH), 6.96 - 6.22 (m, br.; 4H; BQ ArH), $3.71-3.23$ (m, br.; 10H; DT aliphatic H; integral includes signal of $\mathrm{H}_{2} \mathrm{O}$ ), $3.21-2.73$ (m, br.; 4H; DT aliphatic $\mathrm{H}$ ), $2.11-0.36$ (m, br.; 8H; BQ aliphatic $\mathrm{H}$ ).

3340 (s), 2970 (m), 2928 (m), 2874 (m), 1770 (m), 1749 (m), 1714 (w), 1699 (w), 1591 (w), 1475 (s), 1412 (m), 1364 (m), 1257 (s), 1232 (s), $1190(\mathrm{~m}), 1092(\mathrm{~s}), 957(\mathrm{~m}), 862(\mathrm{w}), 782(\mathrm{w}), 733(\mathrm{w}), 652(\mathrm{w})$.

MALDI-TOF-MS calculated $(\mathrm{m} / \mathrm{z}):[\mathrm{M}(4 \mathrm{AA}+4 \mathrm{BB})+\mathrm{Na}]^{+}=1833.5$

found $(\mathrm{m} / \mathrm{z}):[\mathrm{M}(4 \mathrm{AA}+4 \mathrm{BB})+\mathrm{Na}]^{+}=1833.9$
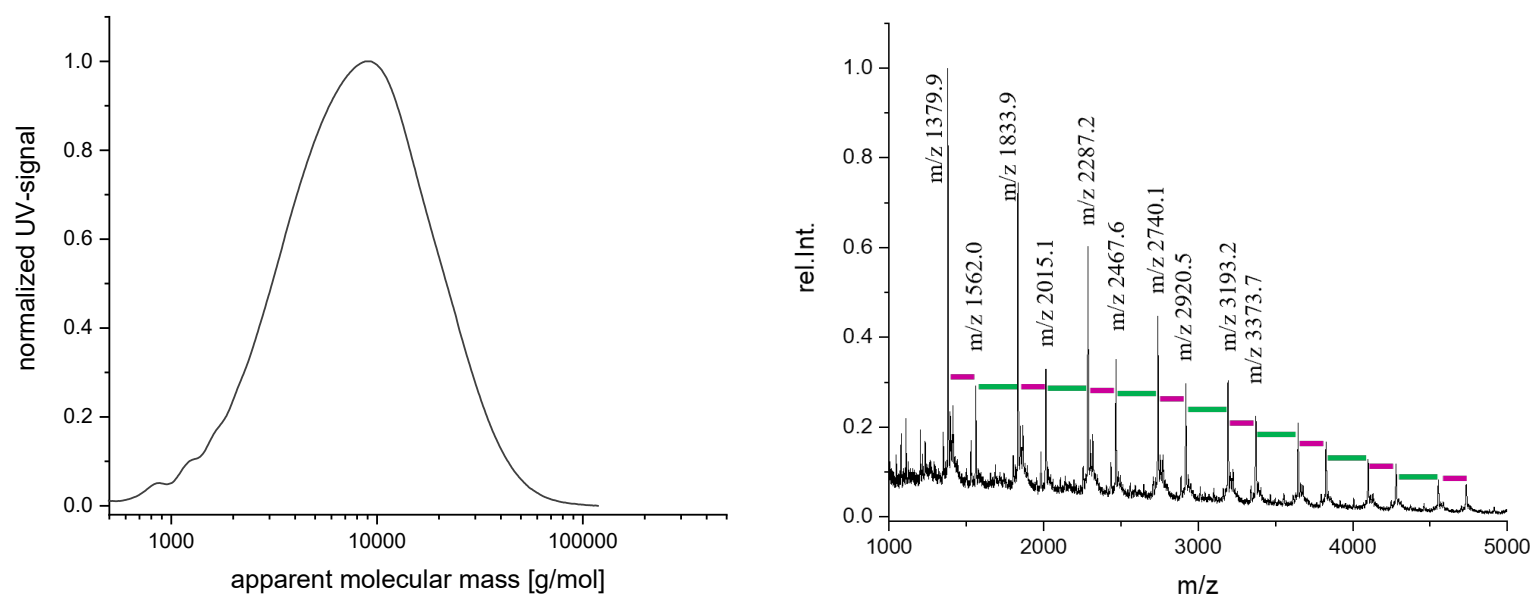

Figure S 33. p(BQB-EDET) analysis. GPC chromatogram at $280 \mathrm{~nm}$ (left) and MALDI-TOF-MS spectrum (right, $\mathrm{m} / \mathrm{z}$ values were assigned to $\mathrm{Na}+$ ion adducts of the oligomer showing the homologous raw of $\mathrm{AA}$ and $\mathrm{BB}$ monomer additions in the TCC-polymer product. Colored bars represent the $\mathrm{m} / \mathrm{z}$ differences corresponding to the repeating units, where green refers to biscatechols and purple to dithiols). 
<smiles>CCC(C)(CC)C1=CC(=O)C(=O)C=C1</smiles>

Yield

GPC

$\mathrm{T}_{\mathrm{g}}$

TGA

${ }^{1} \mathrm{H}-\mathrm{NMR}(500 \mathrm{MHz})$ in $\mathrm{DMSO}_{6} \mathrm{~d}_{6}$

FT-IR $\left[\mathrm{cm}^{-1}\right]$

$58 \%$

$\mathrm{M}_{w, \text { app }}=14.2 \mathrm{~kg} / \mathrm{mol}, \mathrm{Ð}=2.3, \mathrm{DP}(\mathrm{AA}+\mathrm{BB})=32$

$124{ }^{\circ} \mathrm{C}$

$325^{\circ} \mathrm{C}$ (maximal degradation rate)

$\delta[\mathrm{ppm}] 9.59-8.15$ (m, br.; 4H; BQ ArOH), 7.26 - 6.23 (m, br.; 9H; BQ and DT ArH), 4.24 - 2.90 (m, br.; 5H; DT aliphatic H, includes signal of $\mathrm{H}_{2} \mathrm{O}$ ), $1.90-0.24$ (m, br.; $8 \mathrm{H}$; BQ aliphatic $\mathrm{H}$ ).

3360 (s), 2970 (s), 2935 (m), 1749 (m), 1716 (m), 1699 (m), 1649 (m), 1593 (m), 1541 (w), 1508 (m), 1475 (s), 1410 (m), 1373 (m), $1319(\mathrm{~m}), 1230$ (s), $1138(\mathrm{~m}), 1101(\mathrm{~m}), 957(\mathrm{w}), 862(\mathrm{w}), 730(\mathrm{w})$, $651(\mathrm{w})$.

MALDI-TOF-MS calculated $(\mathrm{m} / \mathrm{z}):[\mathrm{M}(4 \mathrm{AA}+4 \mathrm{BB})+\mathrm{Na}]^{+}=1785$

found $(\mathrm{m} / \mathrm{z}):[\mathrm{M}(4 \mathrm{AA}+4 \mathrm{BB})+\mathrm{Na}]^{+}=1786$
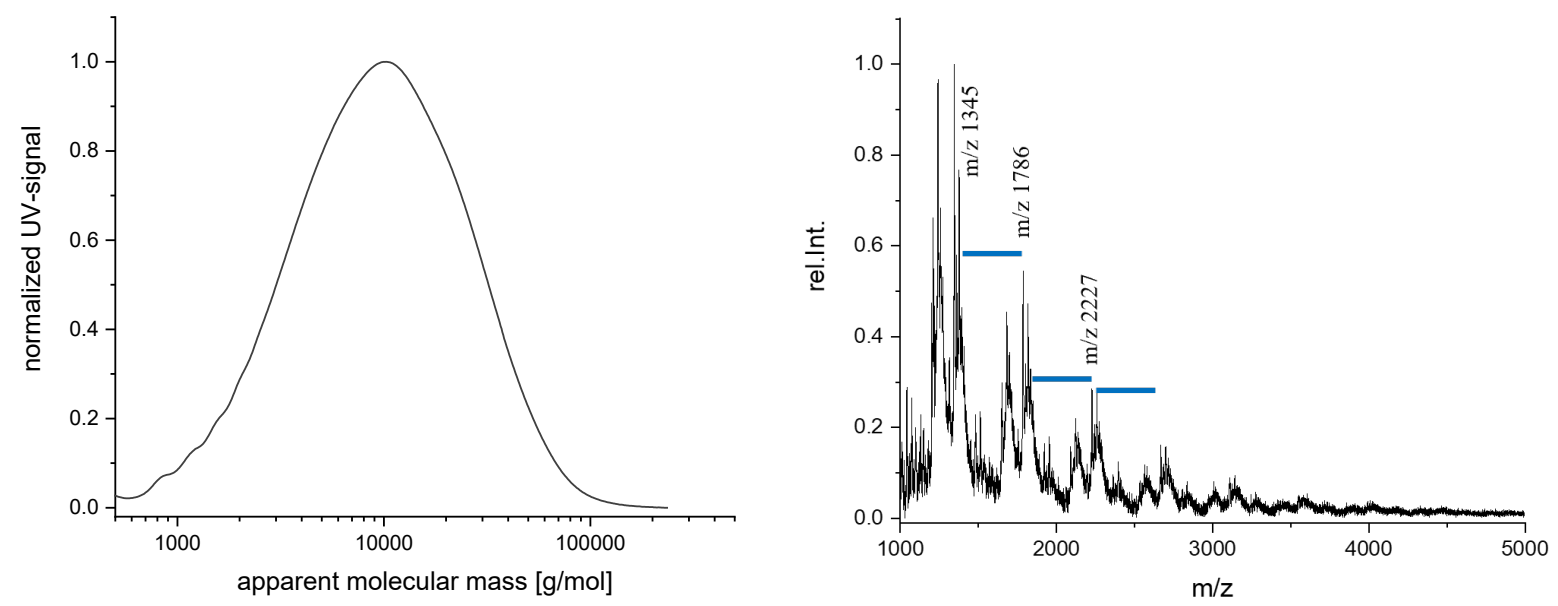

Figure S 34. p(BQB-BMT) analysis. GPC chromatogram at $280 \mathrm{~nm}$ (left) and MALDI-TOF-MS spectrum (right, $\mathrm{m} / \mathrm{z}$ values were assigned to $\mathrm{Na}+$ ion adducts of the oligomer showing the homologous raw of $\mathrm{AA}$ and $\mathrm{BB}$ monomer additions in the TCC-polymer product. Blue colored bars represent the difference corresponding to AABB dimer segments. Despite optimization of matrix and sample preparation means, the TCC polymer shows a challenging desorption behavior, preventing to obtain well resolved MALDI-TOF-MS spectra). 
<smiles>CCC(C)(C1=CC(=O)C(=O)C=C1)c1cc(O)c(O)c(SCC(O)C(O)CSc2cc(C(C)(C)C3=CC(=O)C(=O)C(=O)C3)cc(O)c2O)c1</smiles>

Yield

GPC

$\mathrm{T}_{\mathrm{g}}$

TGA

${ }^{1} \mathrm{H}-\mathrm{NMR}(500 \mathrm{MHz})$ in DMSO-d 6

FT-IR $\left[\mathrm{cm}^{-1}\right]$

$75 \%$

$\mathrm{M}_{w, \text { app }}=3.50 \mathrm{~kg} / \mathrm{mol}, \mathrm{Ð}=1.4, \mathrm{DP}(\mathrm{AA}+\mathrm{BB})=8$

$125^{\circ} \mathrm{C}$

$334{ }^{\circ} \mathrm{C}$ (maximal degradation rate)

$\delta[\mathrm{ppm}] 9.23$ - 8.13 (m, br.; 4H; BQ ArOH), 7.06 - 6.13 (m, br.; 4H; BQ ArH), 5.42 - 4.69 (m, br.; 2H; DT aliphatic H), $3.81-2.56$ (m, br.; 9H; DT aliphatic $\mathrm{H}$; integral includes signal of $\mathrm{H}_{2} \mathrm{O}$ ), $2.00-0.30$ (m, br.; 8H; BQ aliphatic $\mathrm{H}$ ).

3355 (s), 2970 (s), 2930 (m), 1749 (m), 1716 (m), 1699 (m), 1647 (w), 1591 (w), 1475 (m), 1412 (m), 1373 (m), 1230 (s), 1149 (m), $1090(\mathrm{~m}), 1048(\mathrm{~m}), 957(\mathrm{w}), 862(\mathrm{w}), 817(\mathrm{w}), 785(\mathrm{w}), 731(\mathrm{w})$, $655(\mathrm{w})$.

MALDI-TOF-MS calculated $(\mathrm{m} / \mathrm{z}):[\mathrm{M}(4 \mathrm{AA}+4 \mathrm{BB})+\mathrm{Na}]^{+}=1721.4$ found $(\mathrm{m} / \mathrm{z}):[\mathrm{M}(4 \mathrm{AA}+4 \mathrm{BB})+\mathrm{Na}]^{+}=1722.0$
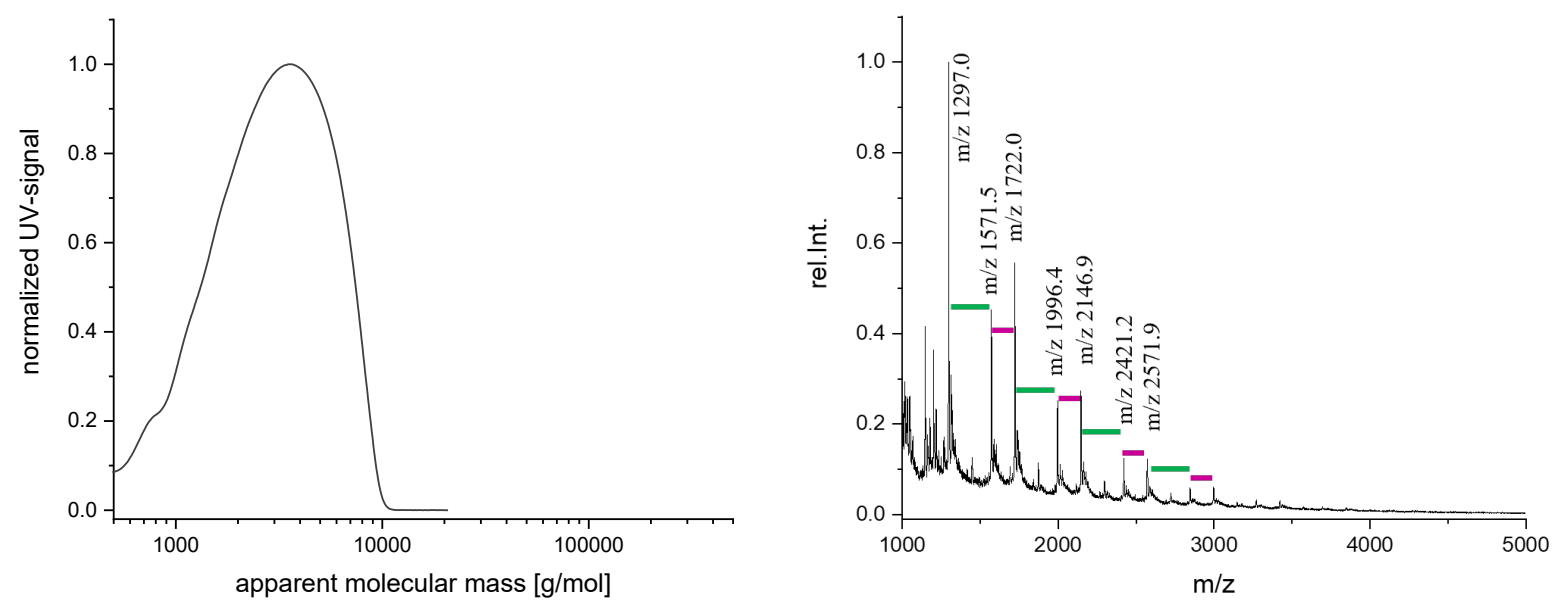

Figure S 35. p(BQB-DTT) analysis. GPC chromatogram at $280 \mathrm{~nm}$ (left) and MALDI-TOF-MS spectrum (right, $\mathrm{m} / \mathrm{z}$ values were assigned to $\mathrm{Na}+$ ion adducts of the oligomer showing the homologous raw of $\mathrm{AA}$ and $\mathrm{BB}$ monomer additions in the TCC-polymer product. Colored bars represent the $\mathrm{m} / \mathrm{z}$ differences corresponding to the repeating units, where green refers to biscatechols and purple to dithiols). 


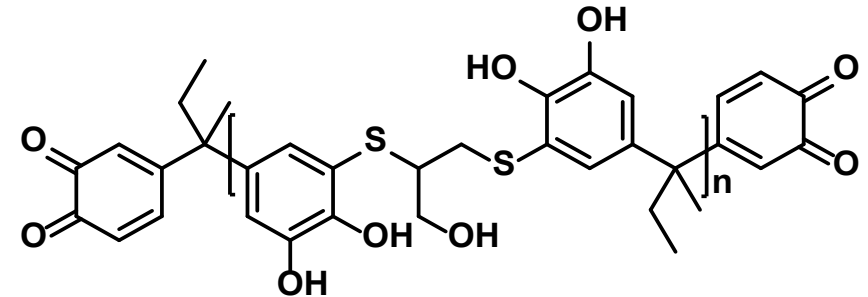

Yield

$62 \%$

GPC

$\mathrm{M}_{w, \text { app }}=7.67 \mathrm{~kg} / \mathrm{mol}, \mathrm{Ð}=1.9, \mathrm{DP}(\mathrm{AA}+\mathrm{BB})=19$

$\mathrm{T}_{\mathrm{g}}$

$110^{\circ} \mathrm{C}$

TGA

$321{ }^{\circ} \mathrm{C}$ (maximal degradation rate)

${ }^{1} \mathrm{H}-\mathrm{NMR}(500 \mathrm{MHz}) \quad \delta[\mathrm{ppm}] 9.51-8.07$ (m, br.; 4H; BQ ArOH), $7.14-6.80$ (m, br.; 4H; in $\mathrm{DMSO}_{6}$ BQ ArH), 5.38 - 4.77 (m, br.; 1H; DT aliphatic H), $3.86-2.53$ (m, br.; 9H; DT aliphatic $\mathrm{H}$; integral includes signal of $\left.\mathrm{H}_{2} \mathrm{O}\right), 2.07-0.08$ (m, br.; 8H; BQ aliphatic $\mathrm{H}$ ).

FT-IR $\left[\mathrm{cm}^{-1}\right]$ 3364 (s), 2970 (s), 2935 (m), 1749 (m), 1716 (m), 1699 (m)n 1510 (m), 1475 (s), 1410 (m), 1373 (m), 1232 (s), 1147 (m), 957 (w), 862 (w), $818(\mathrm{w}), 783(\mathrm{w}), 730(\mathrm{w}), 652(\mathrm{w})$.

MALDI-TOF-MS calculated $(\mathrm{m} / \mathrm{z}):[\mathrm{M}(4 \mathrm{AA}+4 \mathrm{BB})+\mathrm{Na}]^{+}=1601$ found $(\mathrm{m} / \mathrm{z}):[\mathrm{M}(4 \mathrm{AA}+4 \mathrm{BB})+\mathrm{Na}]^{+}=1601$
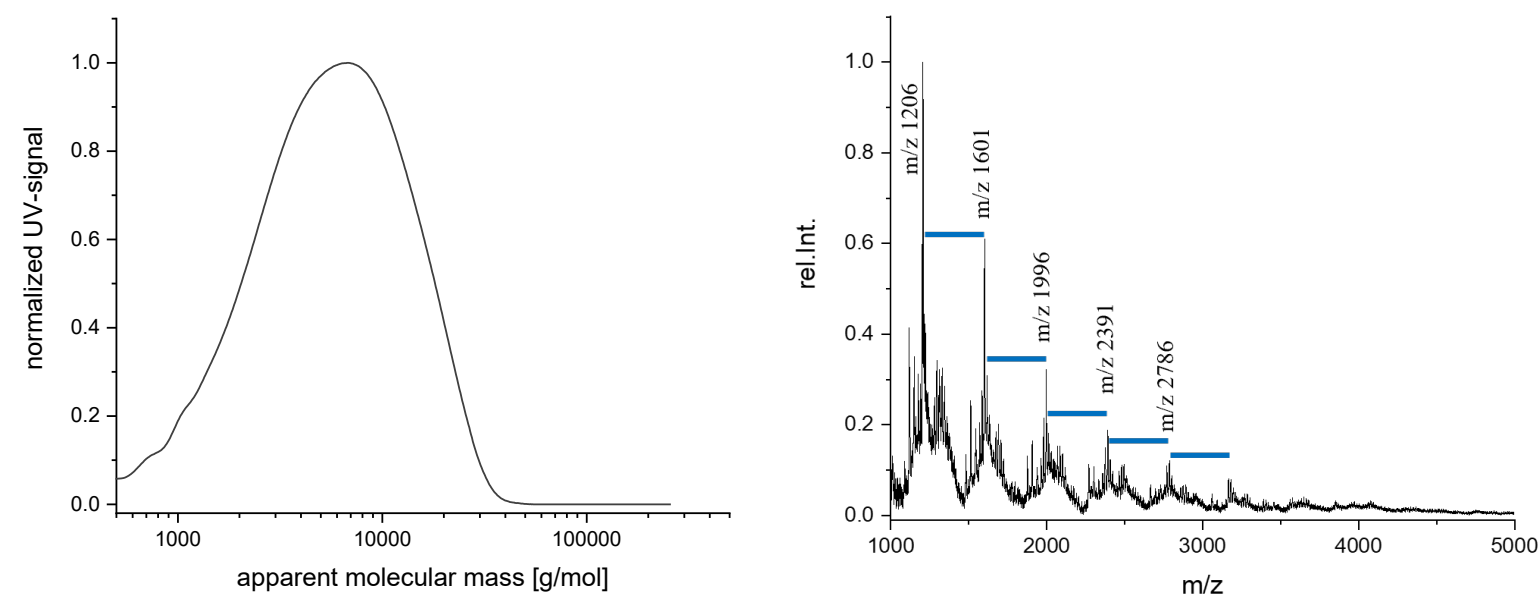

Figure S 36. p(BQB-DMP) analysis. GPC chromatogram at $280 \mathrm{~nm}$ (left) and MALDI-TOF-MS spectrum (right, $\mathrm{m} / \mathrm{z}$ values were assigned to $\mathrm{Na}+$ ion adducts of the oligomer showing the homologous raw of $\mathrm{AA}$ and $\mathrm{BB}$ monomer additions in the TCC-polymer product. Blue colored bars represent the difference corresponding to AABB dimer segments). 
<smiles>CCC(C)(C)c1cc(O)c(O)c(SCC(=O)OCCOC(=O)CSc2cc(C(C)(CC)C3=CC(=O)C(=O)C(=O)C3)cc(O)c2O)c1</smiles>

Yield

GPC

$\mathrm{T}_{\mathrm{g}}$

TGA

${ }^{1} \mathrm{H}-\mathrm{NMR}(500 \mathrm{MHz})$ in $\mathrm{DMSO}_{6} \mathrm{~d}_{6}$

FT-IR $\left[\mathrm{cm}^{-1}\right]$

$59 \%$

$\mathrm{M}_{w, \text { app }}=6.39 \mathrm{~kg} / \mathrm{mol}, \mathrm{Ð}=1.8, \mathrm{DP}(\mathrm{AA}+\mathrm{BB})=13$

$72{ }^{\circ} \mathrm{C}$

$354{ }^{\circ} \mathrm{C}$ (maximal degradation rate)

$\delta[\mathrm{ppm}] 9.86-8.34$ (m, br.; 4H; BQ ArOH), 7.12 - 6.20 (m, br.; 4H; BQ ArH), 4.32 - 3.47 (m, br.; 7H; DT aliphatic H), 3.31 - 3.04 (m, br.; 1H; DT aliphatic H), $2.08-0.34$ (m, br.; 8H; BQ aliphatic H). 3376 (m), 2970 (m), 1736 (s), 1591 (w), 1475 (m), 1412 (m), 1371 (m), 1232 (s), 1122 (m), 1055 (w), 956 (w), 864 (w), 818 (w), 785 (w), $732(\mathrm{w}), 656(\mathrm{w})$.

MALDI-TOF-MS calculated (m/z): $[\mathrm{M}(4 \mathrm{AA}+4 \mathrm{BB})+\mathrm{Na}]^{+}=1945,4$ found $(\mathrm{m} / \mathrm{z}):[\mathrm{M}(4 \mathrm{AA}+4 \mathrm{BB})+\mathrm{Na}]^{+}=1945,5$
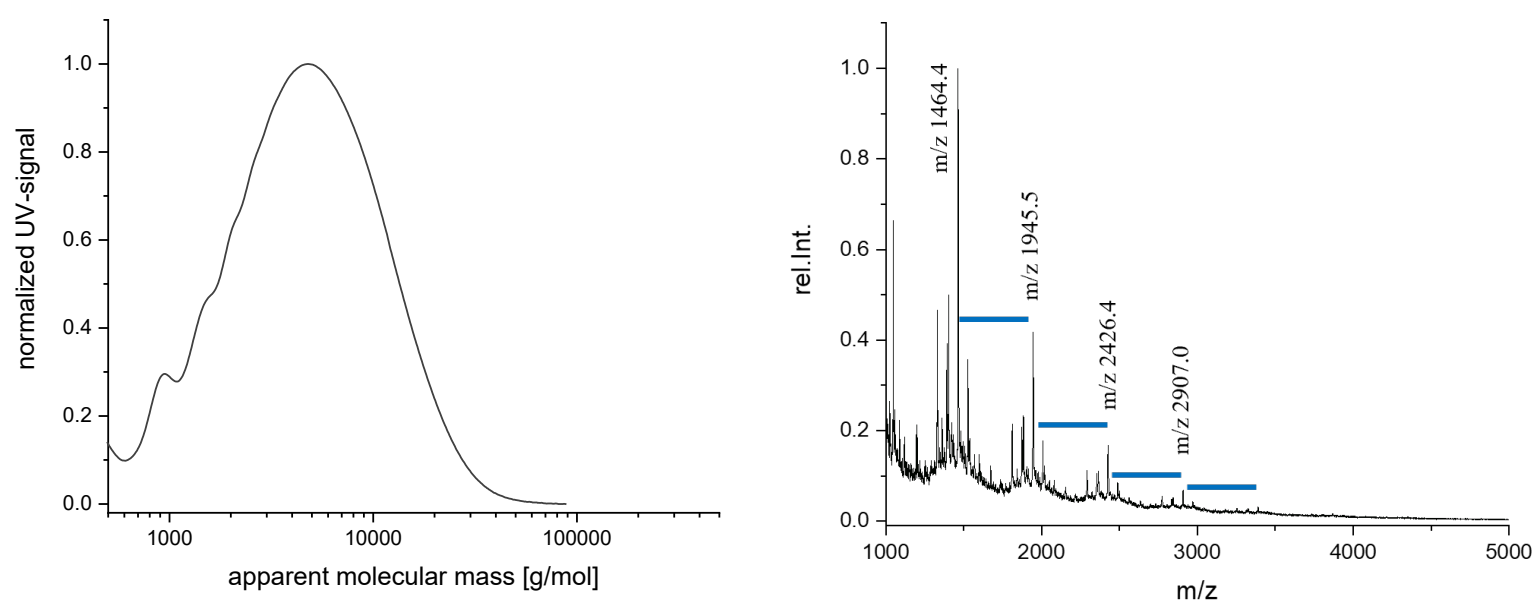

Figure S 37. p(BQB-GDMA) analysis. GPC chromatogram at $280 \mathrm{~nm}$ (left) and MALDI-TOF-MS spectrum (right, $\mathrm{m} / \mathrm{z}$ values were assigned to $\mathrm{Na}+$ ion adducts of the oligomer showing the homologous raw of $\mathrm{AA}$ and $\mathrm{BB}$ monomer additions in the TCC-polymer product. Blue colored bars represent the difference corresponding to AABB dimer segments). 
<smiles>CCC(C)(C1=CC(=O)C(=O)C=C1)c1cc(O)c(O)c(SCCC(=O)OCCOC(=O)CCSc2cc(C(C)(CC)C3=CC(=O)C(=O)C(=O)C3)cc(O)c2O)c1</smiles>

Yield

GPC

$\mathrm{T}_{\mathrm{g}}$

TGA

${ }^{1} \mathrm{H}-\mathrm{NMR}(500 \mathrm{MHz})$ in $\mathrm{DMSO}^{-\mathrm{d}_{6}}$

FT-IR $\left[\mathrm{cm}^{-1}\right]$

$59 \%$

$\mathrm{M}_{w, \text { app }}=12.8 \mathrm{~kg} / \mathrm{mol}, \mathrm{Ð}=1.8, \mathrm{DP}(\mathrm{AA}+\mathrm{BB})=25$

$69^{\circ} \mathrm{C}$

$341{ }^{\circ} \mathrm{C}$ (maximal degradation rate)

$\delta[\mathrm{ppm}] 9.44$ - 8.10 (m, br.; 4H; BQ ArOH), 6.92 - 6.21 (m, br.; 4H; BQ ArH), 4.31 - 4.09 (m, br.; 4H; DT aliphatic H), 3.16-2.09 (m, br.; 12H; DT aliphatic H; integral includes signal of DMSO), 2.00 0.33 (m, br.; 8H; BQ aliphatic $\mathrm{H}$ ).

3377 (m), 2970 (m), 2941 (m), 2880 (w), 1734 (s), 1719 (s), 1591 (w), 1795 (m), 1458 (m), 1410 (m), 1375 (m), 1232 (s), 1184 (s), 1134 (s), 1051 (m), 957 (w), 862 (w), $816(w), 783$ (w), 732 (w), 653 (w).

MALDI-TOF-MS calculated $(\mathrm{m} / \mathrm{z}):[\mathrm{M}(4 \mathrm{AA}+4 \mathrm{BB})+\mathrm{Na}]^{+}=2057.5$ found $(\mathrm{m} / \mathrm{z}):[\mathrm{M}(4 \mathrm{AA}+4 \mathrm{BB})+\mathrm{Na}]^{+}=2057.9$
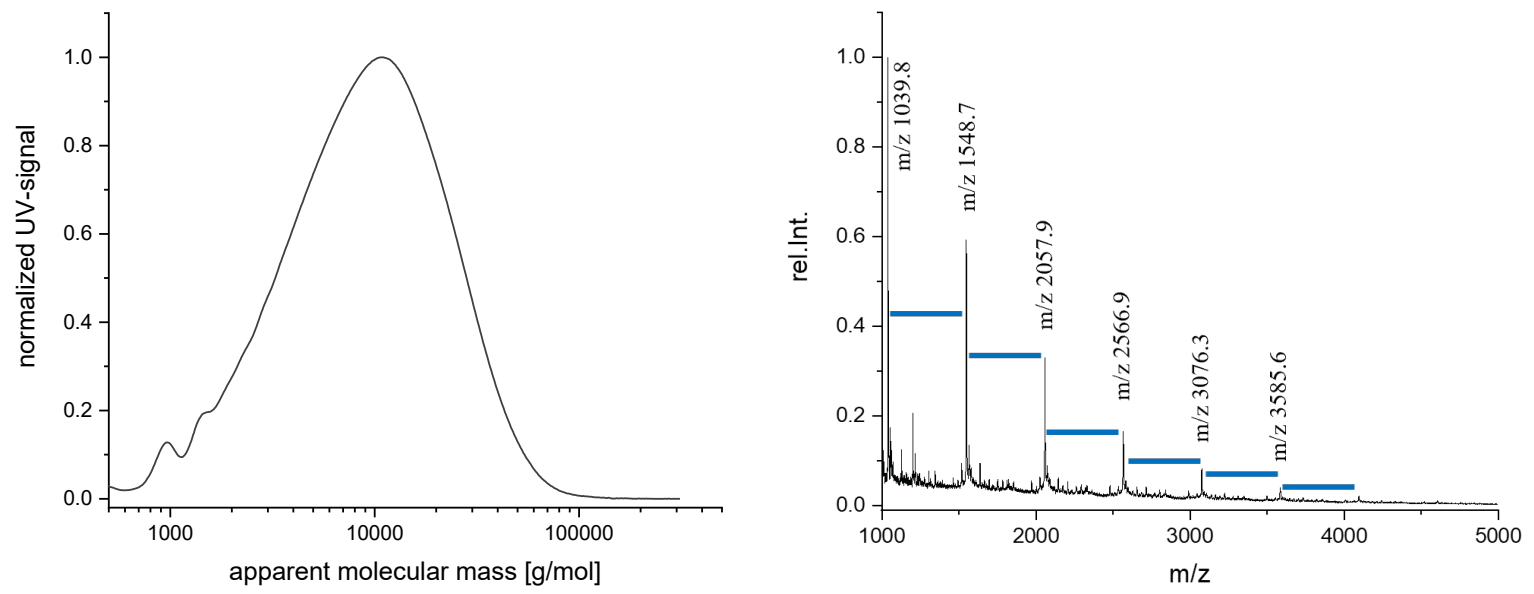

Figure S 38. p(BQB-GDMP) analysis. GPC chromatogram at $280 \mathrm{~nm}$ (left) and MALDI-TOF-MS spectrum (right, $\mathrm{m} / \mathrm{z}$ values were assigned to $\mathrm{Na}+$ ion adducts of the oligomer showing the homologous raw of $\mathrm{AA}$ and $\mathrm{BB}$ monomer additions in the TCC-polymer product. Blue colored bars represent the difference corresponding to AABB dimer segments). 
<smiles>CCC(C)(C1=CC(=O)C(=O)C=C1)c1cc(O)c(O)c(SCCCCSc2cc(C(C)(CC)C3=CC(=O)C(=O)C(=O)C3)cc(O)c2O)c1</smiles>

Yield

GPC

$\mathrm{T}_{\mathrm{g}}$

TGA

${ }^{1} \mathrm{H}-\mathrm{NMR}(500 \mathrm{MHz})$ in DMSO-d 6

FT-IR $\left[\mathrm{cm}^{-1}\right]$

$48 \%$

$\mathrm{M}_{w, \text { app }}=6.04 \mathrm{~kg} / \mathrm{mol}, \mathrm{D}=1.7, \mathrm{DP}(\mathrm{AA}+\mathrm{BB})=15$

$98^{\circ} \mathrm{C}$

$348^{\circ} \mathrm{C}$ (maximal degradation rate)

$\delta[\mathrm{ppm}] 9.45$ - 7.73 (m, br.; 4H; BQ ArOH), 7.12 - 5.98 (m, br.; 4H; BQ ArH), 3.06 - 2.10 (m, br.; 5H; DT aliphatic H; integral includes signal of DMSO), $2.01-0.39$ (m, br.; 12H; BQ and DT aliphatic H). 3340 (s), 2968 (m), 2936 (m), 2875 (w), 1965 (w), 1644 (w), 1593 (m), 1515 (w), 1477 (m), 1412 (m), 1323 (m), 1259 (s), 1230 (s), 1190 (s), 1142 (m), 955 (w), $862(\mathrm{w}), 812(\mathrm{w}), 783(\mathrm{w}), 731(\mathrm{w}), 654$ (w).

MALDI-TOF-MS calculated $(\mathrm{m} / \mathrm{z}):[\mathrm{M}(4 \mathrm{AA}+4 \mathrm{BB})+\mathrm{Na}]^{+}=1593$ found $(\mathrm{m} / \mathrm{z}):[\mathrm{M}(4 \mathrm{AA}+4 \mathrm{BB})+\mathrm{Na}]^{+}=1593$
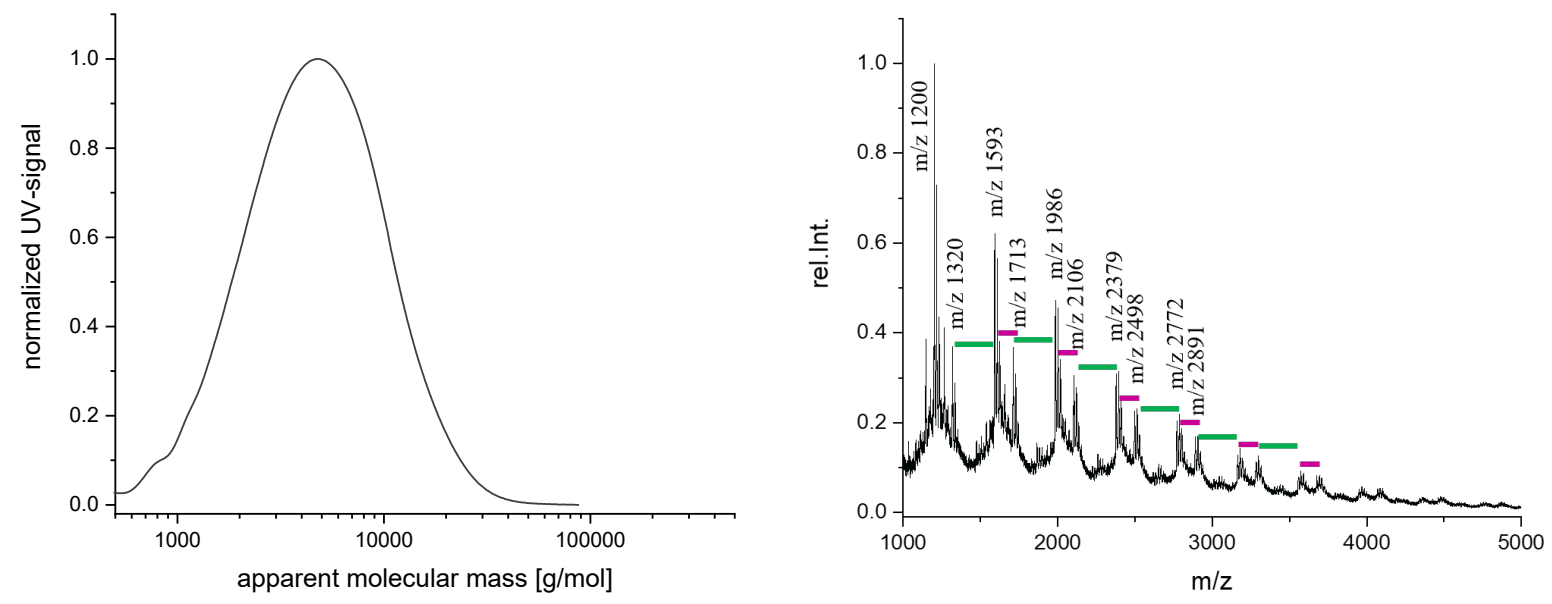

Figure S 39. p(BQB-BDT) analysis. GPC chromatogram at $280 \mathrm{~nm}$ (left) and MALDI-TOF-MS spectrum (right, $\mathrm{m} / \mathrm{z}$ values were assigned to $\mathrm{Na}+$ ion adducts of the oligomer showing the homologous raw of AA and $\mathrm{BB}$ monomer additions in the TCC-polymer product. Colored bars represent the $\mathrm{m} / \mathrm{z}$ differences corresponding to the repeating units, where green refers to biscatechols and purple to dithiols). 
<smiles>CCC(C)(C1=CC(=O)C(=O)C=C1)c1cc(O)c(O)c(SCCCCCCSc2cc(C(C)(CC)C3=CC(O)C(O)C=C3)cc(O)c2O)c1</smiles>

Yield

GPC

$\mathrm{T}_{\mathrm{g}}$

TGA

${ }^{1} \mathrm{H}-\mathrm{NMR}(500 \mathrm{MHz})$ in $\mathrm{DMSO}_{-} \mathrm{d}_{6}$

FT-IR $\left[\mathrm{cm}^{-1}\right]$

$35 \%$

$\mathrm{M}_{w, a p p}=7.68 \mathrm{~kg} / \mathrm{mol}, \mathrm{Ð}=1.7, \mathrm{DP}(\mathrm{AA}+\mathrm{BB})=18$

$78^{\circ} \mathrm{C}$

$352^{\circ} \mathrm{C}$ (maximal degradation rate)

$\delta[\mathrm{ppm}] 9.42$ - 7.90 (m, br.; 4H; BQ ArOH), 7.11 - 5.91 (m, br.; 4H; BQ ArH), $3.01-2.05$ (m, br.; 7H; DT aliphatic H; integral includes signal of DMSO), $2.01-0.32$ (m, br.; 18H; BQ and DT aliphatic H). 3368 (s), 2968 (m), 2927 (s), 2855 (m), 1740 (w), 1701 (w), 1593 (m), 1477 (s), 1412 (s), 1321 (m), 1257 (s), 1229 (s), 1132 (s), 1140 (s), $947(\mathrm{~m}), 858(\mathrm{w}), 802(\mathrm{w}), 781(\mathrm{w}), 729(\mathrm{w}), 654(\mathrm{w})$.

MALDI-TOF-MS calculated $(\mathrm{m} / \mathrm{z}):[\mathrm{M}(4 \mathrm{AA}+4 \mathrm{BB})+\mathrm{Na}]^{+}=1706$ found $(\mathrm{m} / \mathrm{z}):[\mathrm{M}(4 \mathrm{AA}+4 \mathrm{BB})+\mathrm{Na}]^{+}=1706$
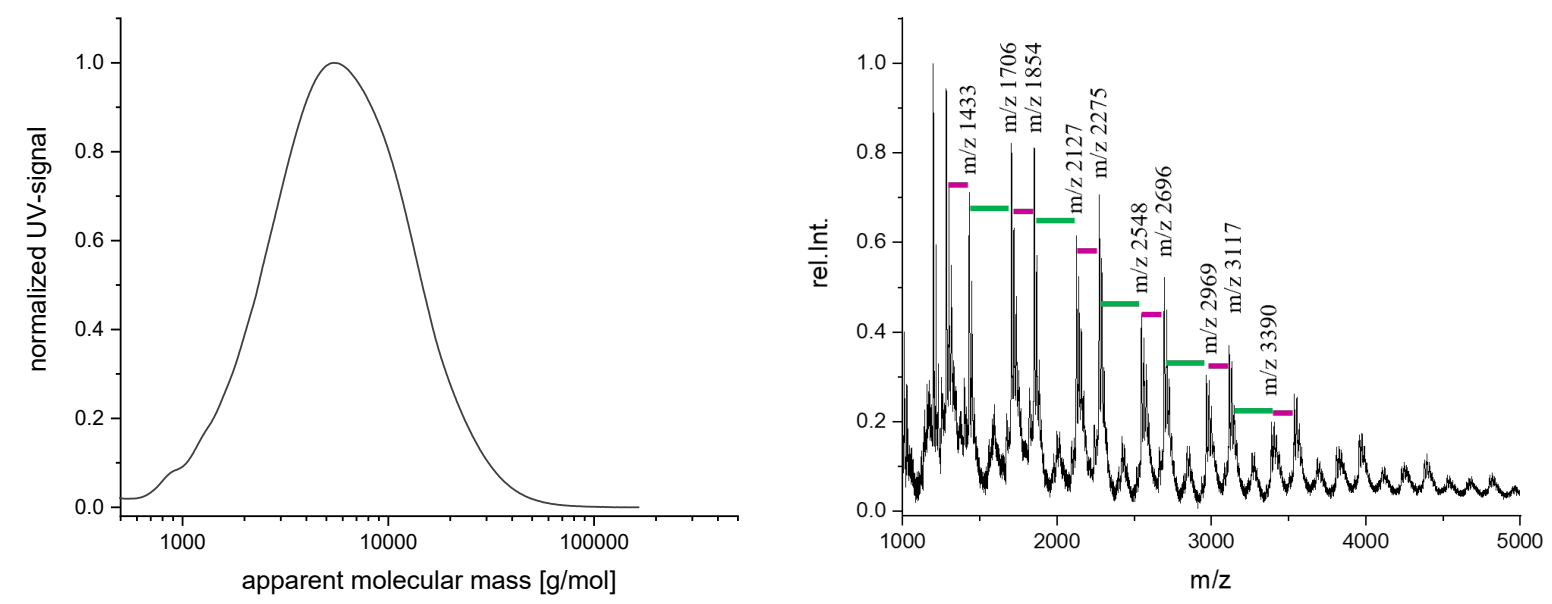

Figure S 40. p(BQB-HDT) analysis. GPC chromatogram at $280 \mathrm{~nm}$ (left) and MALDI-TOF-MS spectrum (right, $\mathrm{m} / \mathrm{z}$ values were assigned to $\mathrm{Na}+$ ion adducts of the oligomer showing the homologous raw of $\mathrm{AA}$ and $\mathrm{BB}$ monomer additions in the TCC-polymer product. Colored bars represent the $\mathrm{m} / \mathrm{z}$ differences corresponding to the repeating units, where green refers to biscatechols and purple to dithiols). 


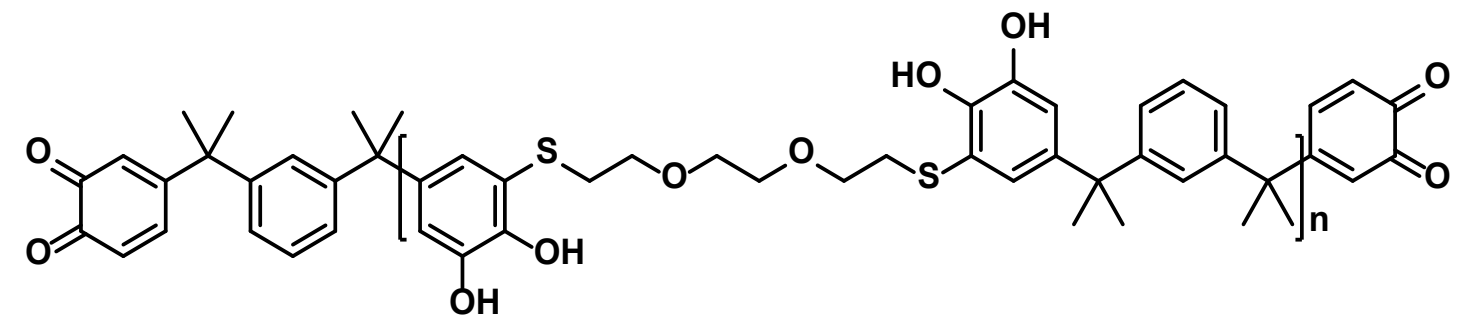

Yield

$46 \%$

GPC

$\mathrm{M}_{w, a p p}=21.7 \mathrm{~kg} / \mathrm{mol}, \mathrm{Ð}=2.5, \mathrm{DP}(\mathrm{AA}+\mathrm{BB})=39$

$\mathrm{T}_{\mathrm{g}}$

$67^{\circ} \mathrm{C}$

TGA

$352{ }^{\circ} \mathrm{C}$ (maximal degradation rate)

${ }^{1} \mathrm{H}-\mathrm{NMR}(500 \mathrm{MHz}) \quad \delta[\mathrm{ppm}] 9.32-8.18$ (m, br.; 4H; BQ ArOH), 7.21 - 6.32 (m, br.; 8H; in $\mathrm{DMSO}-\mathrm{d}_{6}$

BQ ArH), 3.68 - 3.19 (m, br.; 10H; DT aliphatic H; integral includes signal of $\mathrm{H}_{2} \mathrm{O}$ ), $3.14-2.78$ (m, br.; 4H; DT aliphatic $\mathrm{H}$ ), $1.72-1.29$ (m, br.; 12H; BQ aliphatic $\mathrm{H}$ ).

FT-IR $\left[\mathrm{cm}^{-1}\right]$ 3350 (s), 2968 (s), 2928 (s), 2870 (s), 1738 (m), 1593 (m), 1513 (w), 1479 (s), 1414 (m), 1383 (m), 1362 (m), 1271 (s), 1230 (s), 1203 (s), 1163 (m), 1094 (s), 964 (m), 866 (w), 797 (m), 710 (w), 655 (w).

MALDI-TOF-MS calculated $(\mathrm{m} / \mathrm{z}):[\mathrm{M}(4 \mathrm{AA}+4 \mathrm{BB})+\mathrm{Na}]^{+}=2249.8$ found $(\mathrm{m} / \mathrm{z}):[\mathrm{M}(4 \mathrm{AA}+4 \mathrm{BB})+\mathrm{Na}]^{+}=2250.9$
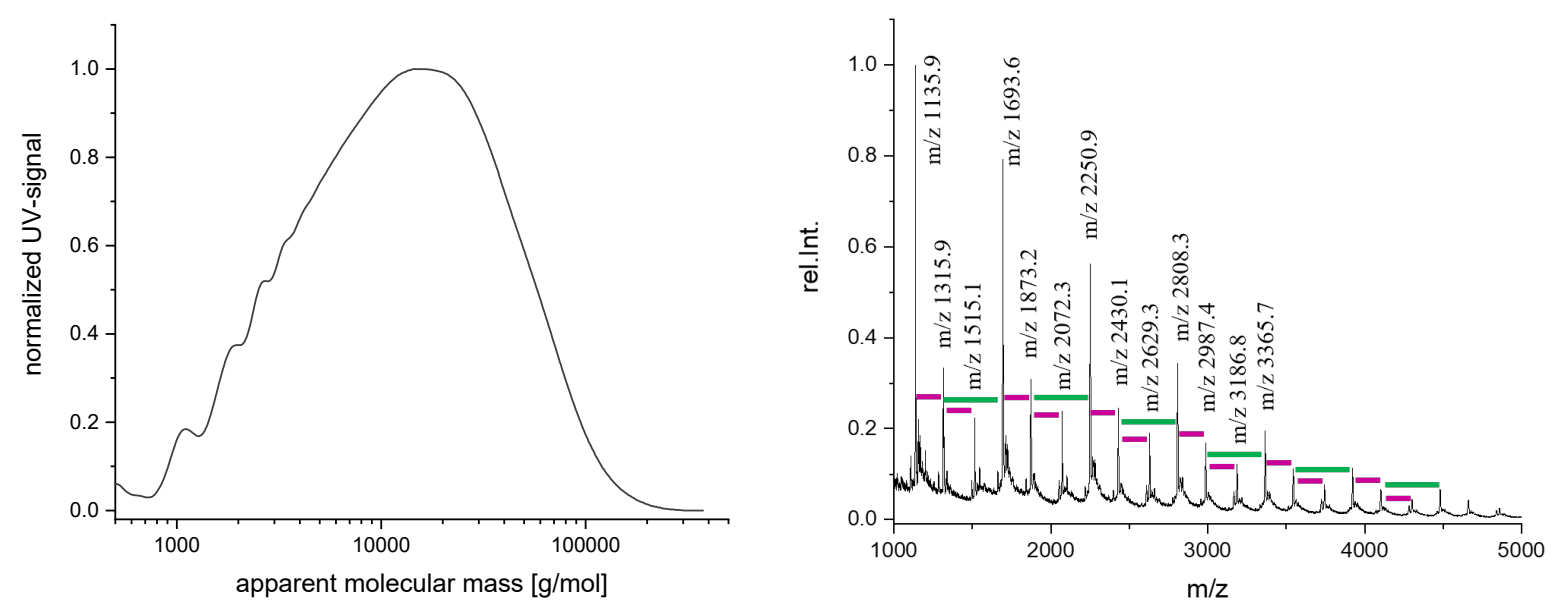

Figure S 41. p(BQM-EDET) analysis. GPC chromatogram at $280 \mathrm{~nm}$ (left) and MALDI-TOF-MS spectrum (right, $\mathrm{m} / \mathrm{z}$ values were assigned to $\mathrm{Na}+$ ion adducts of the oligomer showing the homologous raw of $\mathrm{AA}$ and $\mathrm{BB}$ monomer additions in the TCC-polymer product. Colored bars represent the $\mathrm{m} / \mathrm{z}$ differences corresponding to the repeating units, where green refers to biscatechols and purple to dithiols). 


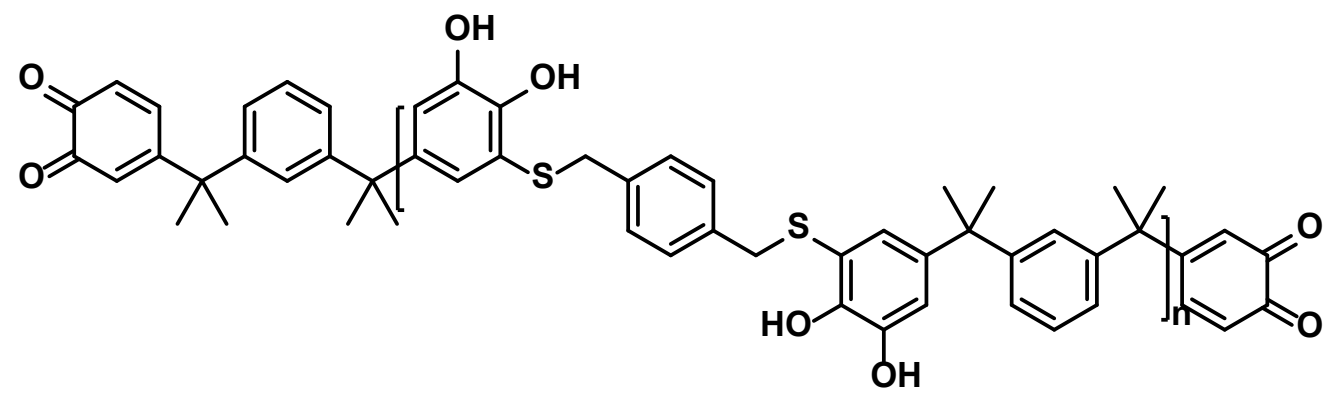

Yield

$61 \%$

GPC

$\mathrm{M}_{w, a p p}=21.1 \mathrm{~kg} / \mathrm{mol}, \mathrm{Ð}=2.4, \mathrm{DP}(\mathrm{AA}+\mathrm{BB})=39$

$\mathrm{T}_{\mathrm{g}}$

$101{ }^{\circ} \mathrm{C}$

TGA

$330{ }^{\circ} \mathrm{C}$ (maximal degradation rate)

${ }^{1} \mathrm{H}-\mathrm{NMR}(500 \mathrm{MHz}) \quad \delta[\mathrm{ppm}] 9.56-8.31$ (m, br.; 4H; BQ ArOH), $7.35-6.23(\mathrm{~m}$, br.; 12H;

in $\mathrm{DMSO}_{6}$

BQ and DT ArH), 4.24 - 3.72 (m, br.; 3H; DT aliphatic H), 1.80 -

1.19 (m, br.; 12H; BQ aliphatic H).

FT-IR $\left[\mathrm{cm}^{-1}\right]$

3380 (s), 2968 (s) 2932 (m), $2872(\mathrm{w}), 1755$ (w), $1651(\mathrm{w}), 1595(\mathrm{~m})$,

1479 (s), 1412 (s), 1362 (m), 1269 (s), 1232 (s), 1201 (s), 1155 (s),

$1092(w), 966(w), 864(w), 825(w), 797(w), 710(w), 657(w)$.

MALDI-TOF-MS calculated $(\mathrm{m} / \mathrm{z}):[\mathrm{M}(4 \mathrm{AA}+4 \mathrm{BB})+\mathrm{Na}]^{+}=2202$

found $(\mathrm{m} / \mathrm{z}):[\mathrm{M}(4 \mathrm{AA}+4 \mathrm{BB})+\mathrm{Na}]^{+}=2203$
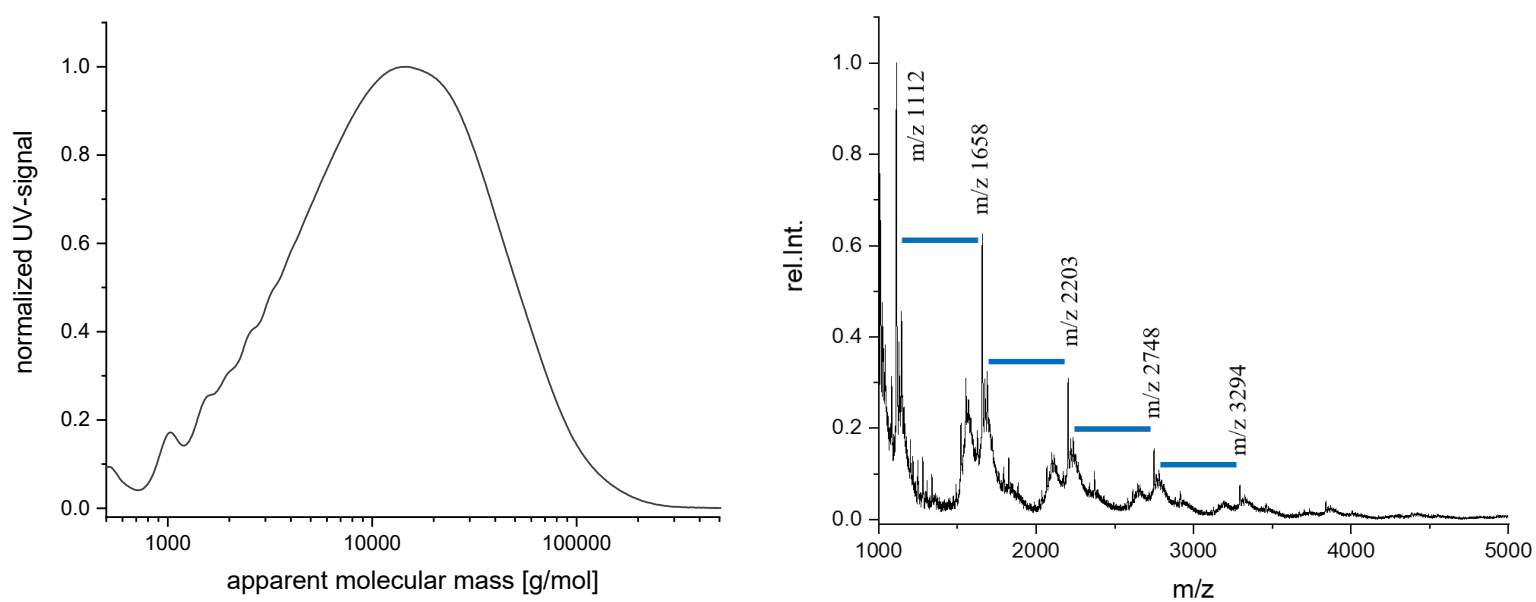

Figure S 42. p(BQM-BMT) analysis. GPC chromatogram at $280 \mathrm{~nm}$ (left) and MALDI-TOF-MS spectrum (right, $\mathrm{m} / \mathrm{z}$ values were assigned to $\mathrm{Na}+$ ion adducts of the oligomer showing the homologous raw of AA and BB monomer additions in the TCC-polymer product. Blue colored bars represent the difference corresponding to AABB dimer segments. Despite optimization of matrix and sample preparation means, the TCC polymer shows a challenging desorption behavior, preventing to obtain well resolved MALDI-TOF-MS spectra). 


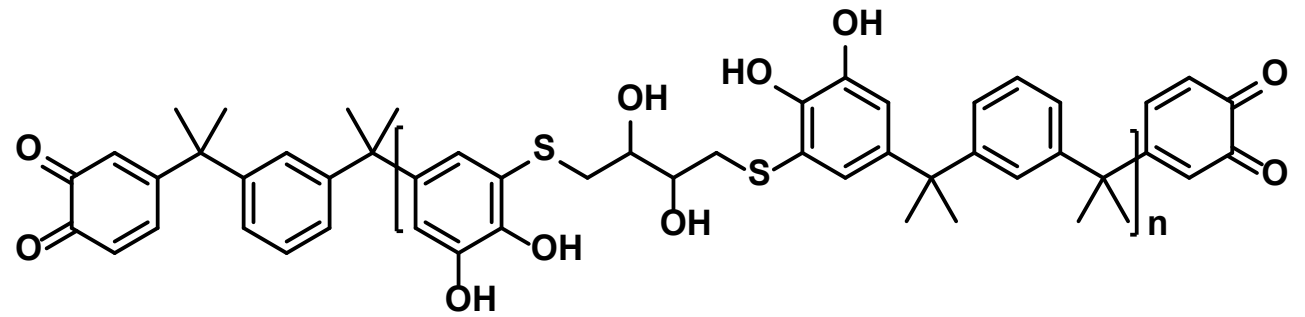

$58 \%$

Yield

GPC

$\mathrm{T}_{\mathrm{g}}$

TGA

${ }^{1} \mathrm{H}-\mathrm{NMR}(500 \mathrm{MHz})$ in $\mathrm{DMSO}_{-} \mathrm{d}_{6}$

FT-IR $\left[\mathrm{cm}^{-1}\right]$
$\mathrm{M}_{w, a p p}=11.8 \mathrm{~kg} / \mathrm{mol}, \mathrm{Ð}=1.9, \mathrm{DP}(\mathrm{AA}+\mathrm{BB})=22$

$109^{\circ} \mathrm{C}$

$339{ }^{\circ} \mathrm{C}$ (maximal degradation rate)

$\delta[\mathrm{ppm}] 9.27$ - 8.34 (m, br.; 4H; BQ ArOH), 7.27 - 6.28 (m, br.; 8H; BQ ArH), 5.33 - 4.82 (m, br.; 2H; DT aliphatic H), $3.78-2.57$ (m, br.; 7H; DT aliphatic $\mathrm{H}$; integral includes signal of $\mathrm{H}_{2} \mathrm{O}$ ), $1.85-1.21$ (m, br.; 12H; BQ aliphatic H).

3359 (s), 2968 (s), 2930 (m), 2874 (w), 1757 (w), 1699 (w), 1646 (w), 1595 (m), 1479 (s), 1412 (m), 1384 (m), 1362 (m), 1273 (s), 1232 (s), 1207 (s), 1163 (m), 1083 (m), 1057 (m), 966 (w), 927 (w), 864 (w), $796(\mathrm{w}), 710(\mathrm{w}), 653(\mathrm{w})$.

MALDI-TOF-MS calculated $(\mathrm{m} / \mathrm{z}):[\mathrm{M}(4 \mathrm{AA}+4 \mathrm{BB})+\mathrm{Na}]^{+}=2137.7$ found $(\mathrm{m} / \mathrm{z}):[\mathrm{M}(4 \mathrm{AA}+4 \mathrm{BB})+\mathrm{Na}]^{+}=2138.7$
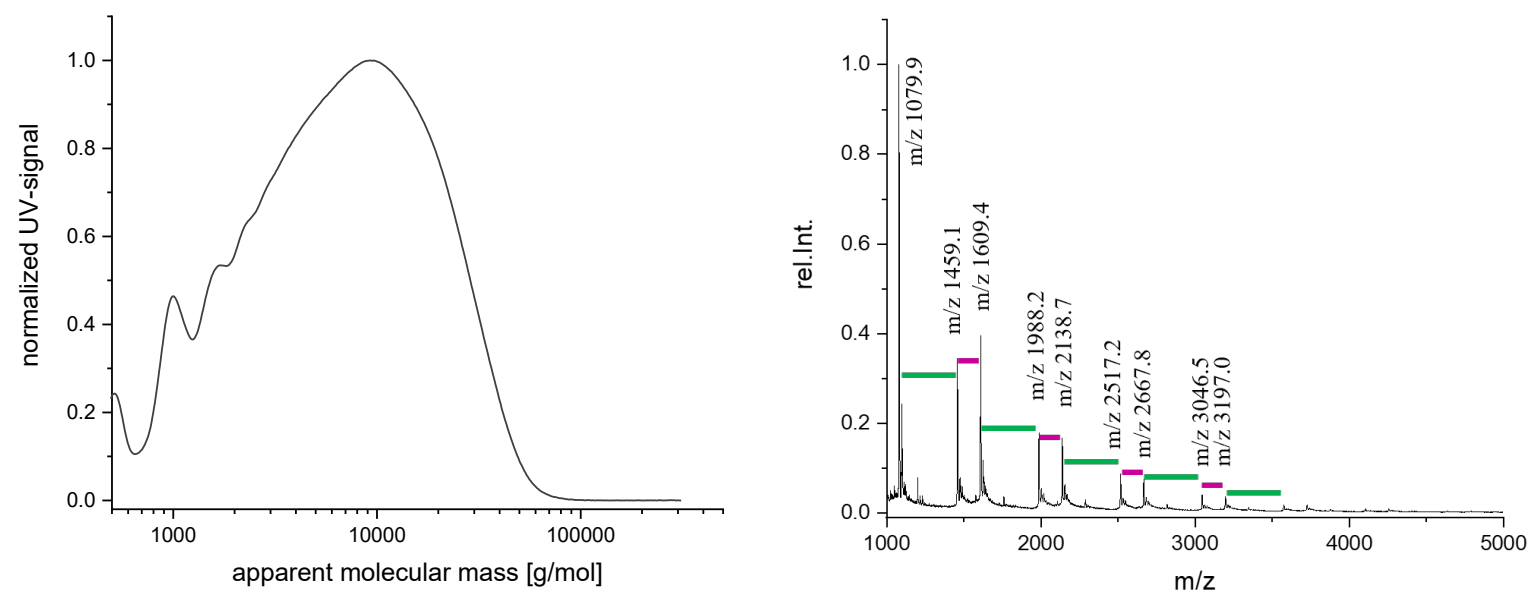

Figure S 43. p(BQM-DTT) analysis. GPC chromatogram at $280 \mathrm{~nm}$ (left) and MALDI-TOF-MS spectrum (right, $\mathrm{m} / \mathrm{z}$ values were assigned to $\mathrm{Na}+$ ion adducts of the oligomer showing the homologous raw of AA and $\mathrm{BB}$ monomer additions in the TCC-polymer product. Colored bars represent the $\mathrm{m} / \mathrm{z}$ differences corresponding to the repeating units, where green refers to biscatechols and purple to dithiols). 


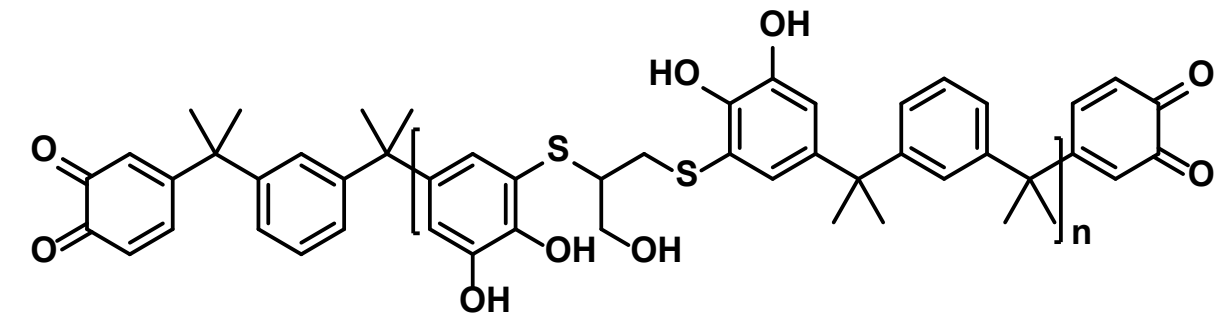

Yield

GPC

$\mathrm{T}_{\mathrm{g}}$

TGA

${ }^{1} \mathrm{H}-\mathrm{NMR}(500 \mathrm{MHz})$ in $\mathrm{DMSO}_{-} \mathrm{d}_{6}$

FT-IR $\left[\mathrm{cm}^{-1}\right]$

$29 \%$

$\mathrm{M}_{w, a p p}=26.0 \mathrm{~kg} / \mathrm{mol}, \mathrm{Ð}=2.0, \mathrm{DP}(\mathrm{AA}+\mathrm{BB})=52$

$112{ }^{\circ} \mathrm{C}$

$324{ }^{\circ} \mathrm{C}$ (maximal degradation rate)

$\delta[\mathrm{ppm}] 9.32$ - 8.26 (m, br.; 4H; BQ ArOH), 7.35 - 6.28 (m, br.; 8H; BQ ArH), 5.38 - 5.02 (m, br.; 1H; DT aliphatic H), $3.58-3.48$ (m, br.; 1H; DT aliphatic H), 3.26 - 2.79 (m, br.; 3H; DT aliphatic H), $1.69-1.30$ (m, br.; 12H; BQ aliphatic H).

3373 (s), 2968 (s), 2934 (m), 2874 (w), 1753 (w), 1734 (w), 1651 (w), 1591 (m), 1479 (s), 1412 (m), 1364 (m), 1271 (s), 1230 (s), 1205 (s), $1157(\mathrm{~m}), 1092(\mathrm{w}), 964(\mathrm{w}), 866(\mathrm{w}), 798(\mathrm{w}), 710(\mathrm{w}), 650(\mathrm{w})$.

MALDI-TOF-MS calculated $(\mathrm{m} / \mathrm{z}):[\mathrm{M}(4 \mathrm{AA}+4 \mathrm{BB})+\mathrm{Na}]^{+}=2017.6$ found $(\mathrm{m} / \mathrm{z}):[\mathrm{M}(4 \mathrm{AA}+4 \mathrm{BB})+\mathrm{Na}]^{+}=2018.0$
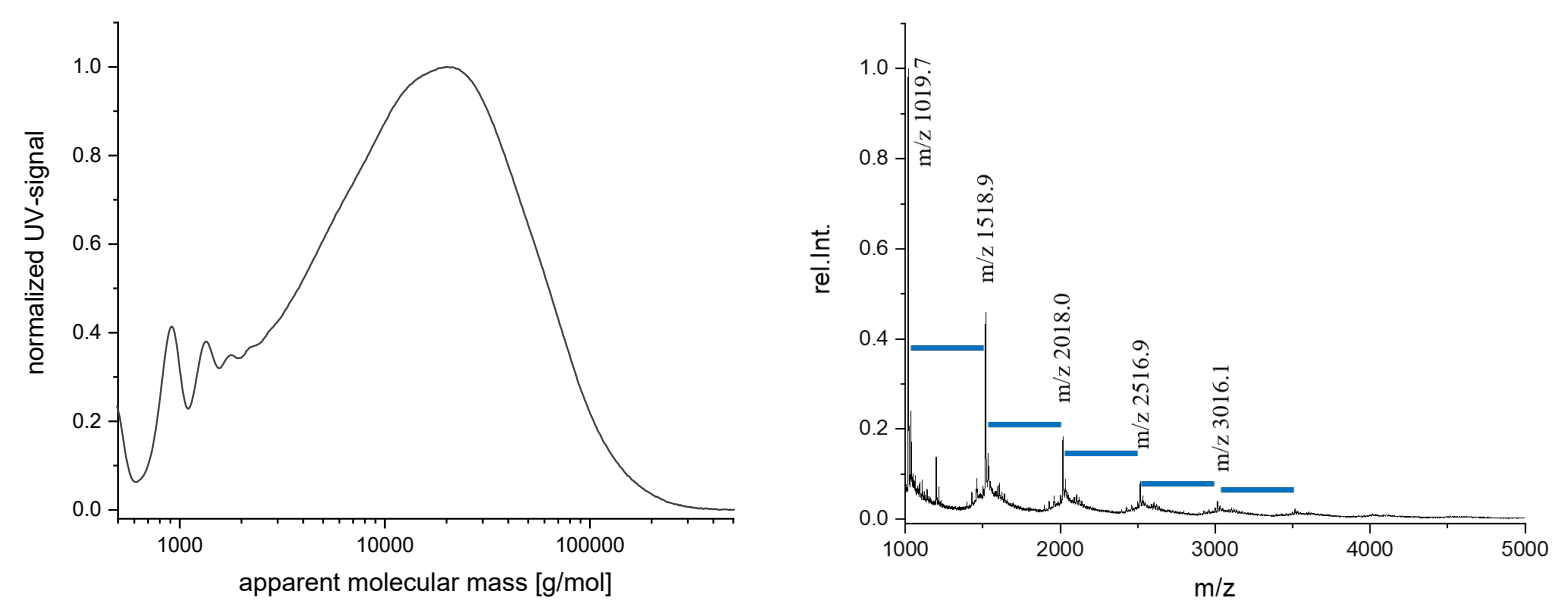

Figure S 44. p(BQM-DMP) analysis. GPC chromatogram at $280 \mathrm{~nm}$ (left) and MALDI-TOF-MS spectrum (right, $\mathrm{m} / \mathrm{z}$ values were assigned to $\mathrm{Na}+$ ion adducts of the oligomer showing the homologous raw of $\mathrm{AA}$ and $\mathrm{BB}$ monomer additions in the TCC-polymer product. Blue colored bars represent the difference corresponding to AABB dimer segments). 


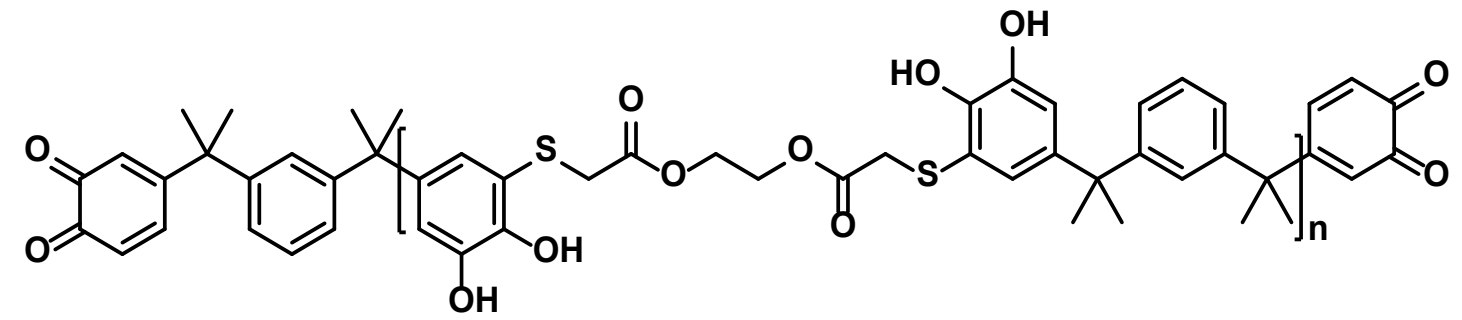

Yield

$25 \%$

GPC

$\mathrm{M}_{w, a p p}=13.9 \mathrm{~kg} / \mathrm{mol}, \mathrm{Ð}=2.0, \mathrm{DP}(\mathrm{AA}+\mathrm{BB})=24$

$\mathrm{T}_{\mathrm{g}}$

$73^{\circ} \mathrm{C}$

TGA

$365{ }^{\circ} \mathrm{C}$ (maximal degradation rate)

${ }^{1} \mathrm{H}-\mathrm{NMR}(500 \mathrm{MHz}) \quad \delta[\mathrm{ppm}] 9.86-8.36$ (m, br.; 3H; BQ ArOH), 7.32 - 6.24 (m, br.; 8H; in $\mathrm{DMSO}_{-} \mathrm{d}_{6}$

BQ ArH), 4.32 - 3.41 (m, br.; 7H; DT aliphatic H), $3.08-2.84$ (m, br.; 1H; DT aliphatic H), 1.80 - 1.24 (m, br.; 12H; BQ aliphatic H).

FT-IR $\left[\mathrm{cm}^{-1}\right]$

3389 (m), 2970 (m), 1738 (s), 1593 (w), 1481 (m), 1413 (m), 1366

(m), 1269 (s), 1232 (s), 1157 (m), 1132 (m), 1055 (w), 966 (w), 891

(w), $864(\mathrm{w}), 798(\mathrm{w}), 710(\mathrm{w}), 652(\mathrm{w})$.

MALDI-TOF-MS calculated $(\mathrm{m} / \mathrm{z}):[\mathrm{M}(4 \mathrm{AA}+4 \mathrm{BB})+\mathrm{Na}]^{+}=2361.6$

found $(\mathrm{m} / \mathrm{z}):[\mathrm{M}(4 \mathrm{AA}+4 \mathrm{BB})+\mathrm{Na}]^{+}=2363.0$
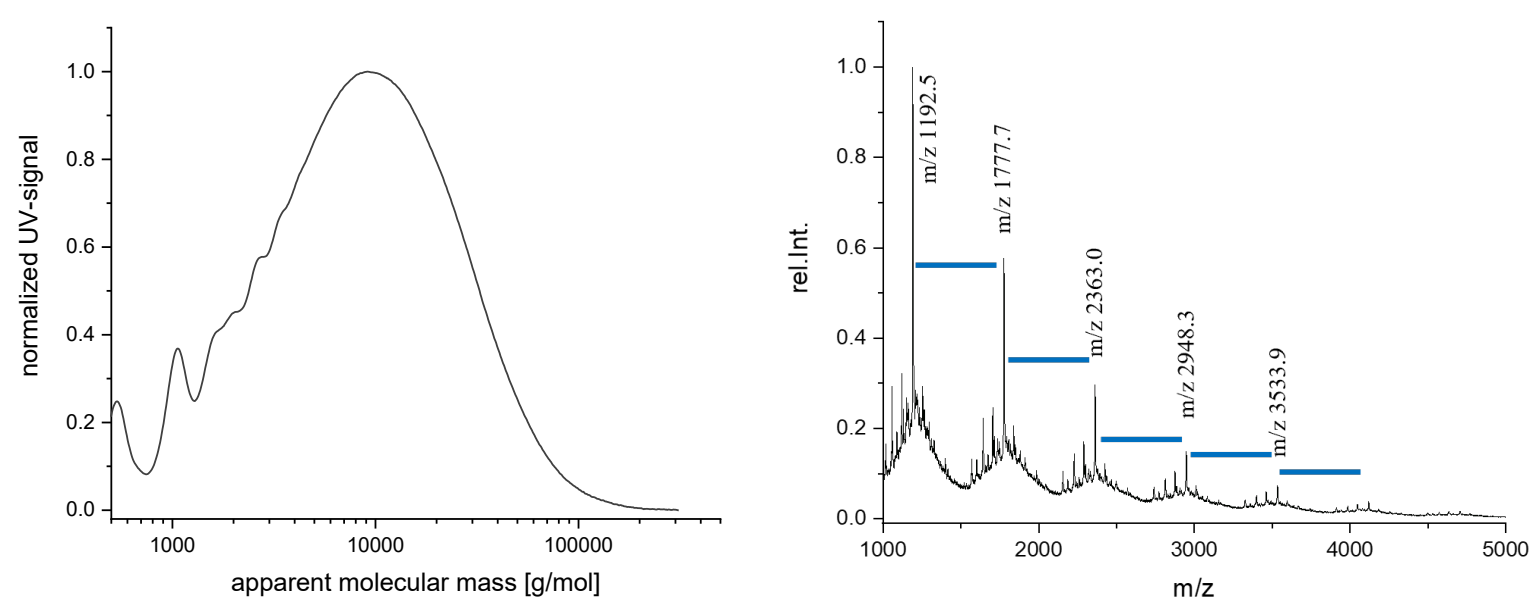

Figure S 45. p(BQM-GDMA) analysis. GPC chromatogram at $280 \mathrm{~nm}$ (left) and MALDI-TOF-MS spectrum (right, $\mathrm{m} / \mathrm{z}$ values were assigned to $\mathrm{Na}+$ ion adducts of the oligomer showing the homologous raw of $\mathrm{AA}$ and $\mathrm{BB}$ monomer additions in the TCC-polymer product. Blue colored bars represent the difference corresponding to AABB dimer segments). 
<smiles>CC(C)(C1=CC(=O)C(=O)C=C1)c1cccc(C(C)(C)c2cccc(C(C)(C)c3cccc(C(C)(C)c4cc(O)c(O)c(SCCC(=O)OCCOC(=O)CCSc5cc(C(C)(C)C6=CC(=O)C(=O)C(=O)C6)cc(O)c5O)c4)c3)c2)c1</smiles>

Yield $\quad 49 \%$

GPC $\quad \mathrm{M}_{w, a p p}=18.5 \mathrm{~kg} / \mathrm{mol}, \mathrm{Ð}=2.0, \mathrm{DP}(\mathrm{AA}+\mathrm{BB})=30$

$\mathrm{T}_{\mathrm{g}}$

$62{ }^{\circ} \mathrm{C}$

TGA

$356{ }^{\circ} \mathrm{C}$ (maximal degradation rate)

${ }^{1} \mathrm{H}-\mathrm{NMR}(500 \mathrm{MHz}) \quad \delta[\mathrm{ppm}] 9.43$ - 8.24 (m, br.; 4H; BQ ArOH), 7.29 - 6.35 (m, br.; 8H; in $\mathrm{DMSO}_{-} \mathrm{d}_{6}$

BQ ArH), 4. $34-3.85$ (m, br.; 4H; DT aliphatic H), 3.06- 2.82 (m, br.; 3H; DT aliphatic H), 2.67 - 1.94 (m, br.; 6H; DT aliphatic H), $1.70-1.23$ (m, br.; 12H; BQ aliphatic H).

FT-IR [ $\left.\mathrm{cm}^{-1}\right] \quad 3389(\mathrm{~m}), 2970(\mathrm{~m}), 1736(\mathrm{~s}), 1593(\mathrm{w}), 1479(\mathrm{~m}), 1412(\mathrm{~m}), 1381$ (m), 1366 (m), 1234 (s), 1136 (s), 1055 (w), 964 (w), 930 (w), 864 (w), $830(\mathrm{w}), 798(\mathrm{w}), 710(\mathrm{w}), 654(\mathrm{w})$.

MALDI-TOF-MS calculated $(\mathrm{m} / \mathrm{z}):[\mathrm{M}(4 \mathrm{AA}+4 \mathrm{BB})+\mathrm{Na}]^{+}=2473.7$

found $(\mathrm{m} / \mathrm{z}):[\mathrm{M}(4 \mathrm{AA}+4 \mathrm{BB})+\mathrm{Na}]^{+}=2474.9$
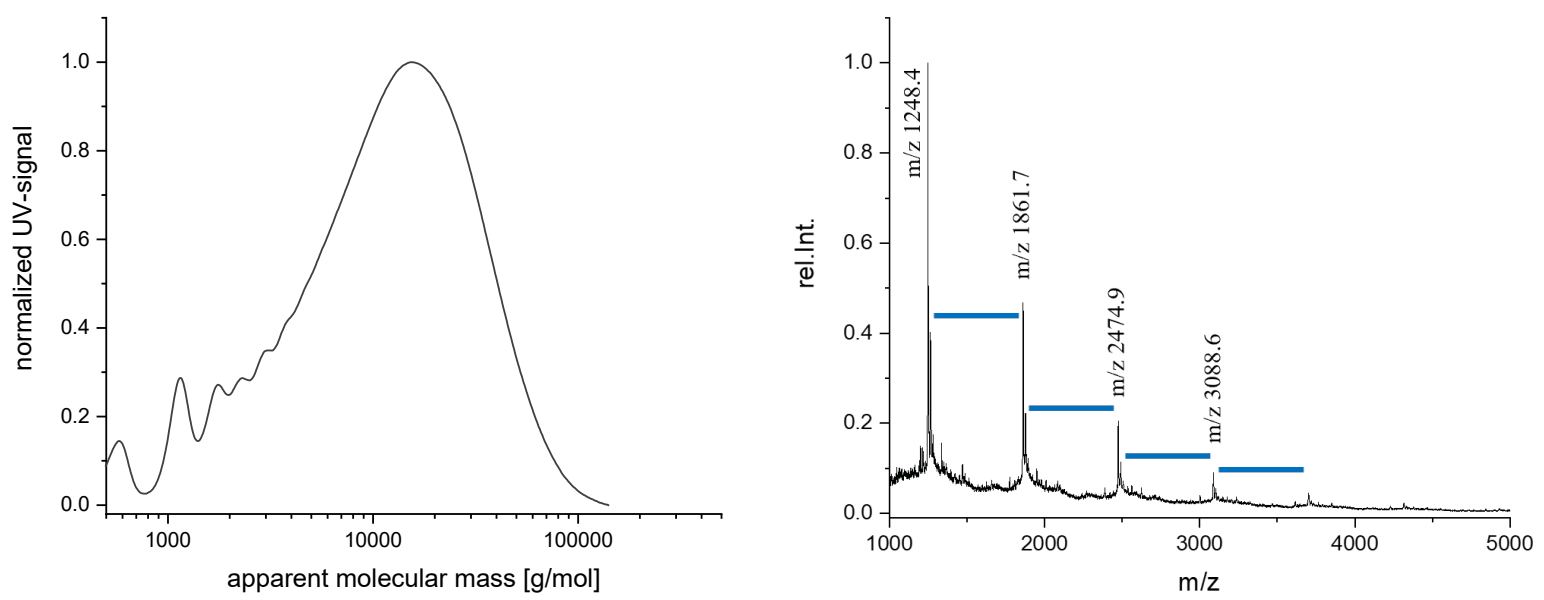

Figure S 46. p(BQM-GDMP) analysis. GPC chromatogram at $280 \mathrm{~nm}$ (left) and MALDI-TOF-MS spectrum (right, $\mathrm{m} / \mathrm{z}$ values were assigned to $\mathrm{Na}+$ ion adducts of the oligomer showing the homologous raw of $\mathrm{AA}$ and $\mathrm{BB}$ monomer additions in the TCC-polymer product. Blue colored bars represent the difference corresponding to AABB dimer segments). 


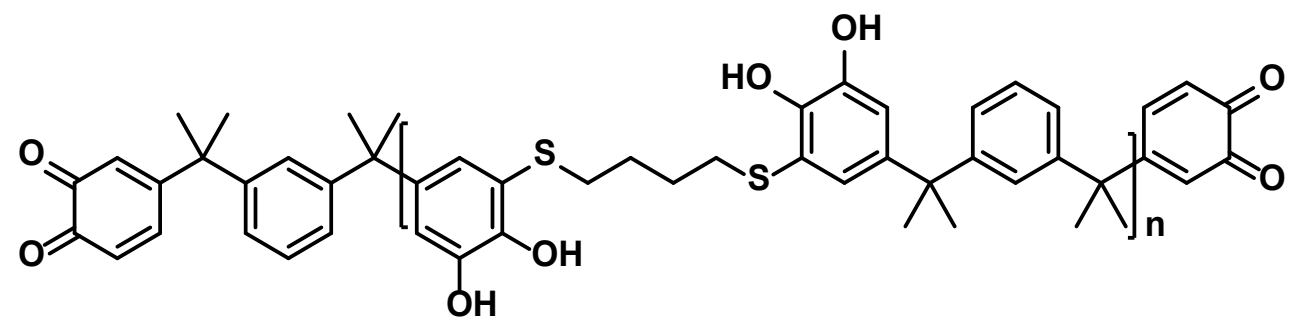
Yield $\quad 48 \%$
GPC $\quad \mathrm{M}_{w, a p p}=7.93 \mathrm{~kg} / \mathrm{mol}, \mathrm{Ð}=2.0, \mathrm{DP}(\mathrm{AA}+\mathrm{BB})=16$
$\mathrm{T}_{\mathrm{g}} \quad 75^{\circ} \mathrm{C}$
TGA $\quad 357^{\circ} \mathrm{C}$ (maximal degradation rate)
${ }^{1} \mathrm{H}-\mathrm{NMR}(500 \mathrm{MHz}) \quad \delta[\mathrm{ppm}] 9.30-8.07$ (m, br.; 4H; BQ ArOH), $7.28-6.28$ (m, br.; 8H; in $\mathrm{DMSO}_{-} \mathrm{d}_{6} \quad$ BQ ArH), $2.94-2.51$ (m, br.; 3H; DT aliphatic H), $1.72-1.03$ (m, br.; 17H; BQ and DT aliphatic $\mathrm{H})$.
FT-IR [cm $\left.{ }^{-1}\right] \quad 3377(\mathrm{~s}), 2964(\mathrm{~s}), 3932(\mathrm{~m}), 2868(\mathrm{w}), 1650(\mathrm{w}), 1595(\mathrm{~m}), 1479(\mathrm{~s})$, 1412 (m), 1360 (m), 1322 (m), 1271 (s), 1223 (s), 1196 (s), 1146 (s), $1090(\mathrm{w}), 966(\mathrm{w}), 930(\mathrm{w}), 866(\mathrm{w}), 825(\mathrm{w}), 797(\mathrm{w}), 731(\mathrm{w}), 710$ (w), $652(\mathrm{w})$.

MALDI-TOF-MS calculated $(\mathrm{m} / \mathrm{z}):[\mathrm{M}(4 \mathrm{AA}+4 \mathrm{BB})+\mathrm{Na}]^{+}=2010$

found $(\mathrm{m} / \mathrm{z}):[\mathrm{M}(4 \mathrm{AA}+4 \mathrm{BB})+\mathrm{Na}]^{+}=2011$
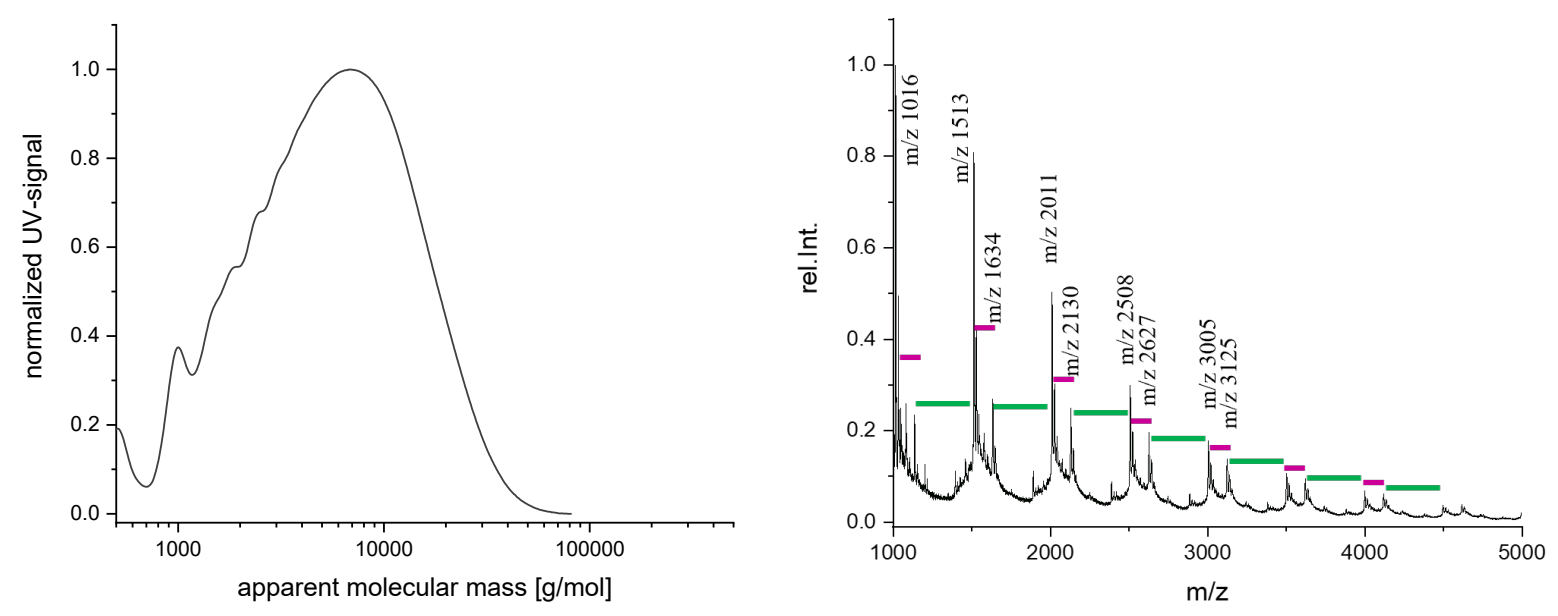

Figure S 47. p(BQM-BDT) analysis. GPC chromatogram at $280 \mathrm{~nm}$ (left) and MALDI-TOF-MS spectrum (right, $\mathrm{m} / \mathrm{z}$ values were assigned to $\mathrm{Na}+$ ion adducts of the oligomer showing the homologous raw of $\mathrm{AA}$ and $\mathrm{BB}$ monomer additions in the TCC-polymer product. Colored bars represent the $\mathrm{m} / \mathrm{z}$ differences corresponding to the repeating units, where green refers to biscatechols and purple to dithiols). 


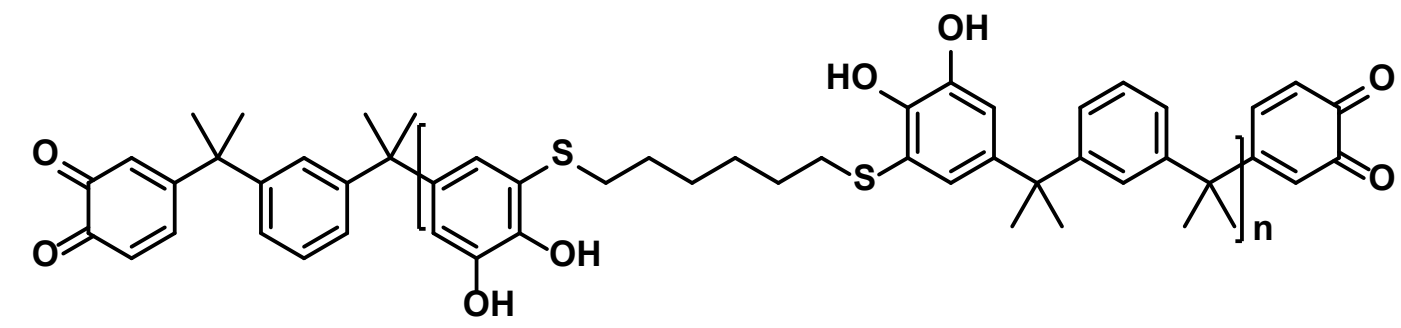
Yield $\quad 61 \%$
GPC $\quad \mathrm{M}_{w, a p p}=12.2 \mathrm{~kg} / \mathrm{mol}, \mathrm{Ð}=2.1, \mathrm{DP}(\mathrm{AA}+\mathrm{BB})=23$
$\mathrm{T}_{\mathrm{g}} \quad 66^{\circ} \mathrm{C}$
TGA $\quad 361{ }^{\circ} \mathrm{C}$ (maximal degradation rate)
${ }^{1} \mathrm{H}-\mathrm{NMR}(500 \mathrm{MHz}) \quad \delta[\mathrm{ppm}] 9.31-8.05$ (m, br.; 4H; BQ ArOH), 7.19 - 6.37 (m, br.; 8H; in $\mathrm{DMSO}_{-} \mathrm{d}_{6} \quad$ BQ ArH), $2.93-2.56$ (m, br.; 6H; DT aliphatic H), 2.18-1.79 (m, br.; 1H; DT aliphatic H), $1.64-0.80$ (m, br.; 23H; BQ and DT aliphatic $\mathrm{H})$.
FT-IR [ $\left.\mathrm{cm}^{-1}\right] \quad 3377(\mathrm{~s}), 2964(\mathrm{~s}), 2932(\mathrm{~s}), 2858(\mathrm{~m}), 1649(\mathrm{w}), 1595(\mathrm{~m}), 1479(\mathrm{~s})$, 1414 (m), 1387 (m), 1360 (m), 1323 (m), 1269 (s), 1229 (s), 1198 (s), $1157(\mathrm{~s}), 1092(\mathrm{w}), 966(\mathrm{w}), 928(\mathrm{w}), 864(\mathrm{w}), 797(\mathrm{w}), 731(\mathrm{w}), 710$ (m), $652(\mathrm{w})$.

MALDI-TOF-MS calculated $(\mathrm{m} / \mathrm{z}):[\mathrm{M}(4 \mathrm{AA}+4 \mathrm{BB})+\mathrm{Na}]^{+}=2122$ found $(\mathrm{m} / \mathrm{z}):[\mathrm{M}(4 \mathrm{AA}+4 \mathrm{BB})+\mathrm{Na}]^{+}=2123$
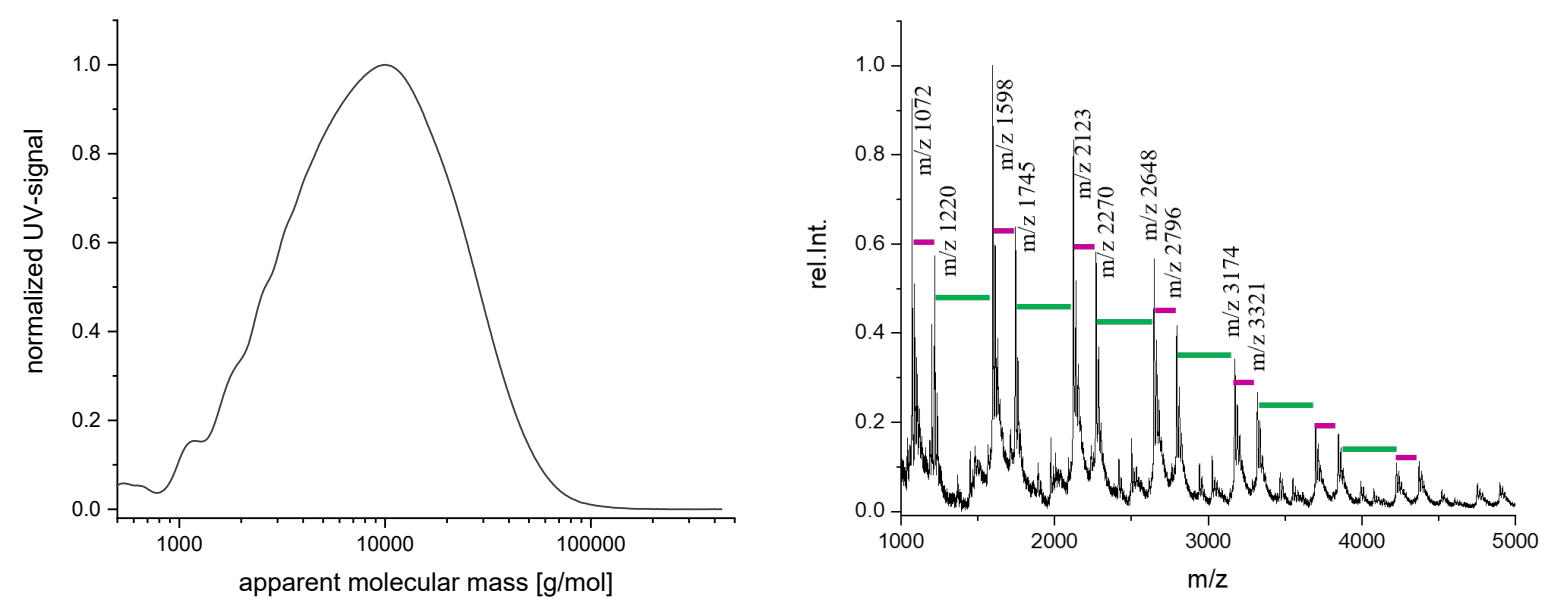

Figure S 48. p(BQM-HDT) analysis. GPC chromatogram at $280 \mathrm{~nm}$ (left) and MALDI-TOF-MS spectrum (right, $\mathrm{m} / \mathrm{z}$ values were assigned to $\mathrm{Na}+$ ion adducts of the oligomer showing the homologous raw of $\mathrm{AA}$ and $\mathrm{BB}$ monomer additions in the TCC-polymer product. Colored bars represent the $\mathrm{m} / \mathrm{z}$ differences corresponding to the repeating units, where green refers to biscatechols and purple to dithiols). 


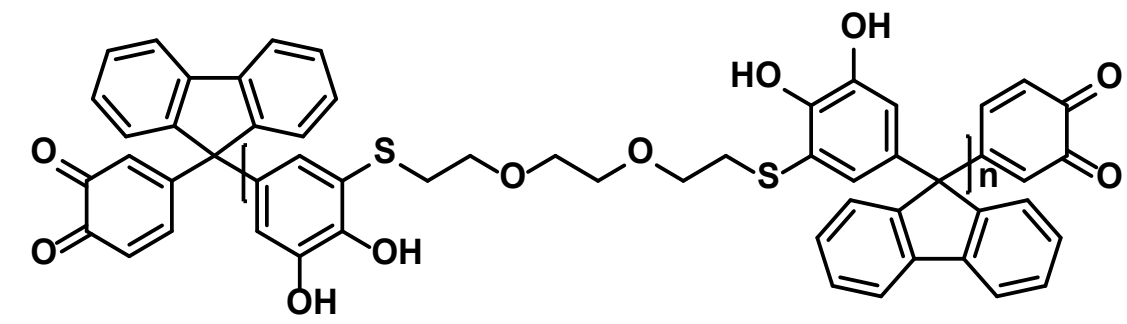

Yield $\quad 58 \%$

GPC $\quad \mathrm{M}_{w, \text { app }}=3.58 \mathrm{~kg} / \mathrm{mol}, \mathrm{D}=1.6, \mathrm{DP}(\mathrm{AA}+\mathrm{BB})=6$

$\mathrm{T}_{\mathrm{g}} \quad 115^{\circ} \mathrm{C}$

TGA $\quad 341{ }^{\circ} \mathrm{C}$ (maximal degradation rate)

${ }^{1} \mathrm{H}-\mathrm{NMR}(500 \mathrm{MHz}) \quad \delta[\mathrm{ppm}] 9.58-8.44$ (m, br.; 4H; BQ ArOH), 7.98 - 6.21 (m, br.; 12H;

in DMSO-d $\quad$ BQ ArH), 3.66-2.71 (m, br.; 14H; DT aliphatic H; integral includes signal of $\mathrm{H}_{2} \mathrm{O}$ ).

FT-IR [ $\left.\mathrm{cm}^{-1}\right] \quad 3320(\mathrm{~m}), 2994(\mathrm{w}), 2913(\mathrm{w}), 2870(\mathrm{w}), 1769(\mathrm{~m}), 1759(\mathrm{~m}), 1699$

(w), $1584(\mathrm{w}), 1473$ (m), 1444 (m), 1409 (m), 1352 (m), 1323 (m),

1244 (s), $1171(\mathrm{~m}), 1095(\mathrm{~m}), 1063(\mathrm{~m}), 900(\mathrm{w}), 850(\mathrm{w}), 751(\mathrm{w})$,

$735(\mathrm{~m}), 655(\mathrm{w})$.

MALDI-TOF-MS calculated $(\mathrm{m} / \mathrm{z}):[\mathrm{M}(4 \mathrm{AA}+4 \mathrm{BB})+\mathrm{Na}]^{+}=2267.8$

found $(\mathrm{m} / \mathrm{z}):[\mathrm{M}(4 \mathrm{AA}+4 \mathrm{BB})+\mathrm{Na}]^{+}=2267.8$
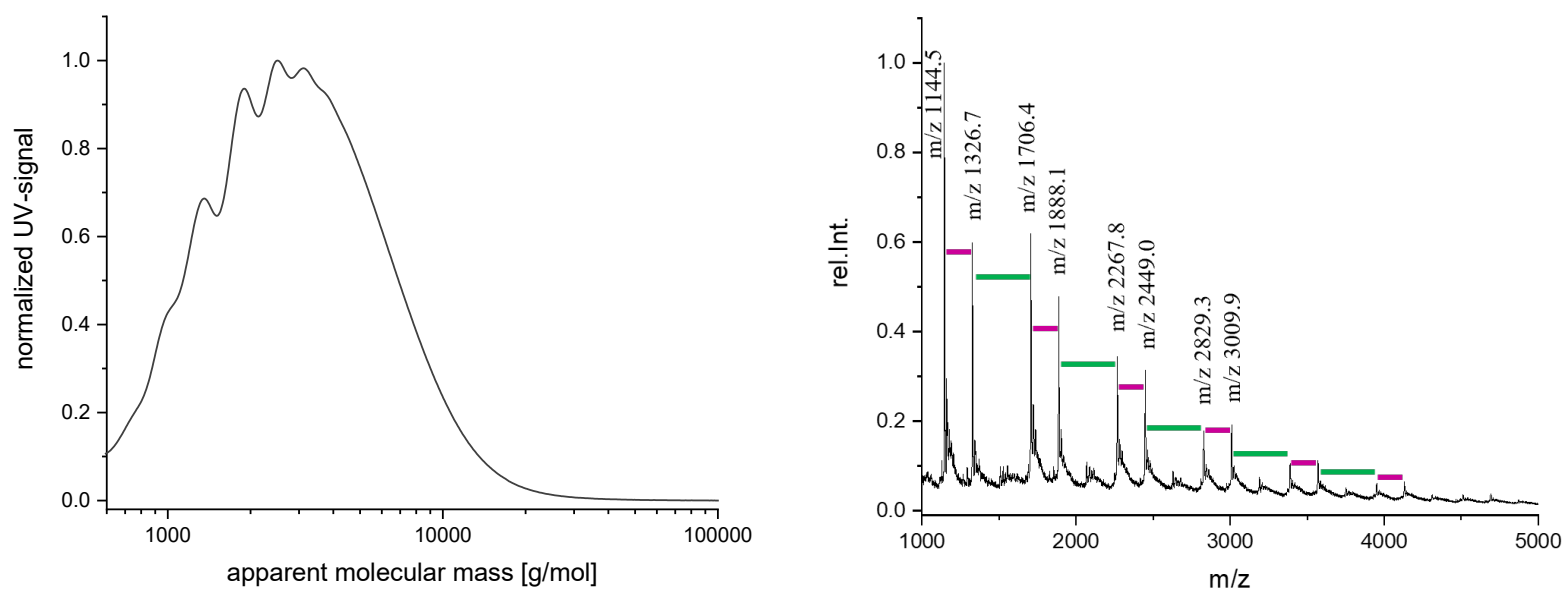

Figure S 49. p(BQFL-EDET) analysis. GPC chromatogram at $280 \mathrm{~nm}$ (left) and MALDI-TOF-MS spectrum (right, $\mathrm{m} / \mathrm{z}$ values were assigned to $\mathrm{Na}+$ ion adducts of the oligomer showing the homologous raw of $\mathrm{AA}$ and $\mathrm{BB}$ monomer additions in the TCC-polymer product. Colored bars represent the $\mathrm{m} / \mathrm{z}$ differences corresponding to the repeating units, where green refers to biscatechols and purple to dithiols). 


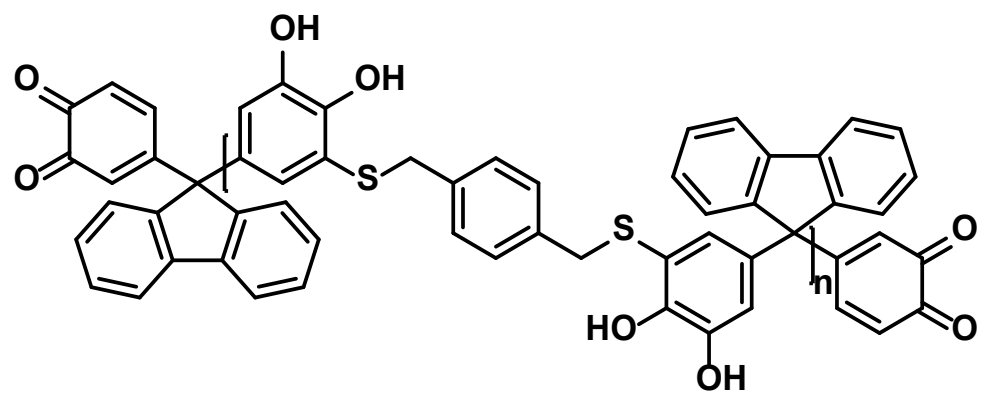

Yield

GPC

$\mathrm{T}_{\mathrm{g}}$

TGA

${ }^{1} \mathrm{H}-\mathrm{NMR}(500 \mathrm{MHz})$ in DMSO-d 6

FT-IR $\left[\mathrm{cm}^{-1}\right]$

$91 \%$

$\mathrm{M}_{w, \text { app }}=4.33 \mathrm{~kg} / \mathrm{mol}, \mathrm{Ð}=1.9, \mathrm{DP}(\mathrm{AA}+\mathrm{BB})=8$

no $\mathrm{T}_{\mathrm{g}}$ detected

$326{ }^{\circ} \mathrm{C}$ (maximal degradation rate)

$\delta[\mathrm{ppm}] 9.79-8.30$ (m, br.; 3H; BQ ArOH), $8.00-6.04$ (m, br.; 12H;

BQ ArH), 4.05 - 3.48 (m, br.; 4H; DT aliphatic H).

3365 (m), 3054 (m), 2995 (m), 1770 (m), 1696 (w), 1645 (w), 1588

(w), 1509 (w), 1476 (m), 1447 (m), 1411 (m), 1356 (m), 1320 (m),

1243 (s), 1167 (m), 1117 (m), 1095 (m), 1059 (w), 1021 (w), 897 (w),

$846(\mathrm{w}), 825(\mathrm{w}), 751(\mathrm{w}), 734(\mathrm{~m}), 698(\mathrm{w}), 657(\mathrm{w}), 619(\mathrm{w})$.

MALDI-TOF-MS calculated $(\mathrm{m} / \mathrm{z}):[\mathrm{M}(4 \mathrm{AA}+4 \mathrm{BB})+\mathrm{Na}]^{+}=2219$

found $(\mathrm{m} / \mathrm{z}):[\mathrm{M}(4 \mathrm{AA}+4 \mathrm{BB})+\mathrm{Na}]^{+}=2219$
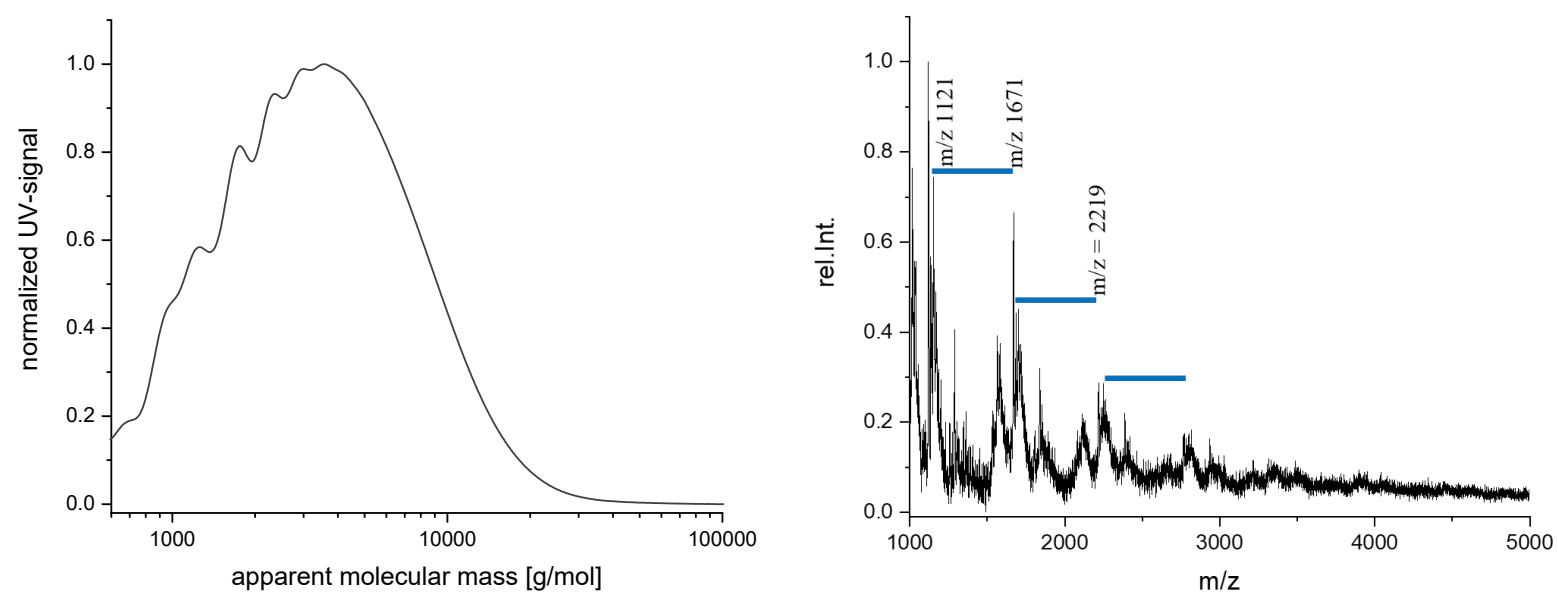

Figure S 50. p(BQFL-BMT) analysis. GPC chromatogram at $280 \mathrm{~nm}$ (left) and MALDI-TOF-MS spectrum (right, $\mathrm{m} / \mathrm{z}$ values were assigned to $\mathrm{Na}+$ ion adducts of the oligomer showing the homologous raw of $\mathrm{AA}$ and $\mathrm{BB}$ monomer additions in the TCC-polymer product. Blue colored bars represent the difference corresponding to AABB dimer segments. Despite optimization of matrix and sample preparation means, the TCC polymer shows a challenging desorption behavior, preventing to obtain well resolved MALDI-TOF-MS spectra). 
<smiles>O=C1C=CC(C2(c3cc(O)c(O)c(SCC(O)C(O)CSc4cc(C5(C6=CC=CC(=O)C(=O)C6)c6ccccc6-c6ccccc65)cc(O)c4O)c3)c3ccccc3-c3ccccc32)=CC1=O</smiles>

Yield

GPC

$\mathrm{T}_{\mathrm{g}}$

TGA

${ }^{1} \mathrm{H}-\mathrm{NMR}(500 \mathrm{MHz})$ in $\mathrm{DMSO}_{-} \mathrm{d}_{6}$

FT-IR $\left[\mathrm{cm}^{-1}\right]$

$78 \%$

$\mathrm{M}_{w, \text { app }}=2.56 \mathrm{~kg} / \mathrm{mol}, \mathrm{Ð}=1.5, \mathrm{DP}(\mathrm{AA}+\mathrm{BB})=5$

$112{ }^{\circ} \mathrm{C}$

$331^{\circ} \mathrm{C}$ (maximal degradation rate)

$\delta[\mathrm{ppm}] 9.52-8.38$ (m, br.; 4H; BQ ArOH), $8.17-6.17$ (m, br.; 12H; BQ ArH), 5.30 - 4.23 (m, br.; 2H; DT aliphatic H), $3.22-2.53$ (m, br.; 3H; DT aliphatic H).

3320 (s), 2995 (m), 2921 (m), 1771 (m), 1761 (m), 1694 (m), 1643 (w), 1588 (m), 1476 (m), 1447 (m), 1412 (m), 1360 (m), 1238 (s), 1168 (m), 1117 (m), 1091 (m), 1056 (m), 898 (w), $850(\mathrm{w}), 750(\mathrm{w})$, $734(\mathrm{~m}), 657(\mathrm{w}), 619(\mathrm{w})$.

MALDI-TOF-MS calculated $(\mathrm{m} / \mathrm{z}):[\mathrm{M}(4 \mathrm{AA}+4 \mathrm{BB})+\mathrm{Na}]^{+}=2155.3$

found $(\mathrm{m} / \mathrm{z}):[\mathrm{M}(4 \mathrm{AA}+4 \mathrm{BB})+\mathrm{Na}]^{+}=2155.5$
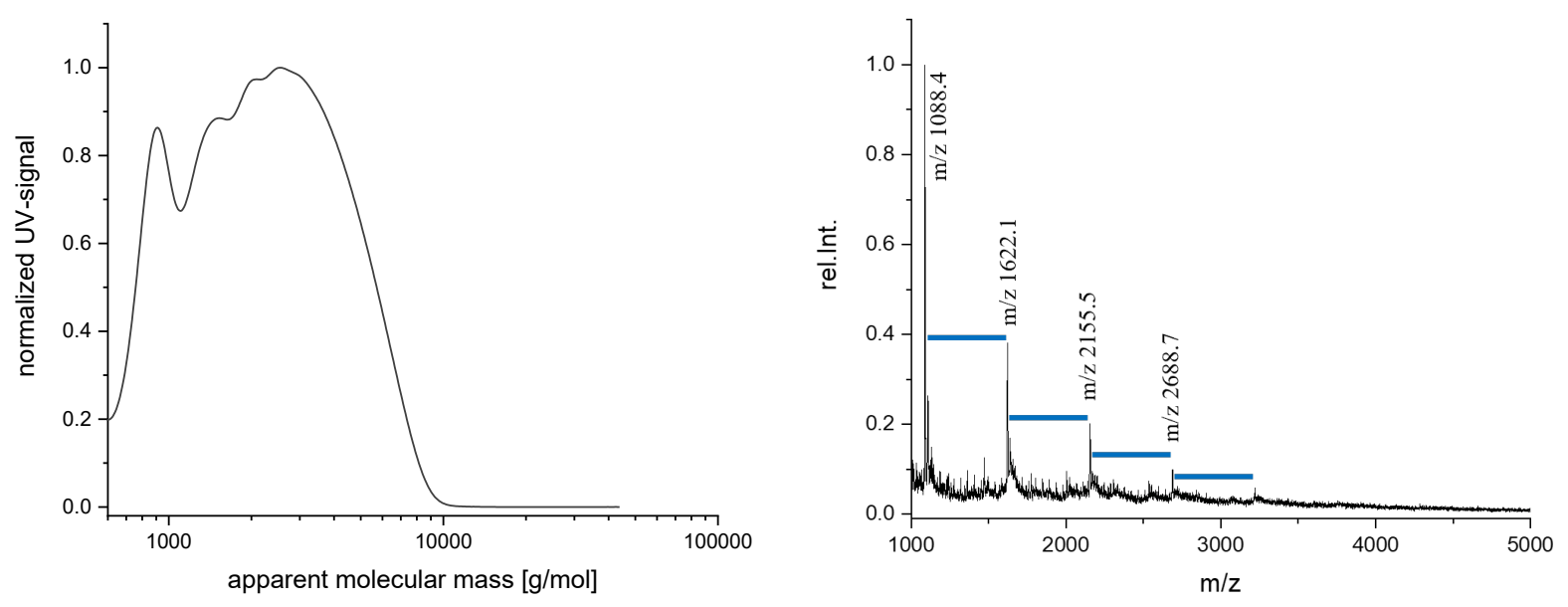

Figure S 51. p(BQFL-DTT) analysis. GPC chromatogram at $280 \mathrm{~nm}$ (left) and MALDI-TOF-MS spectrum (right, $\mathrm{m} / \mathrm{z}$ values were assigned to $\mathrm{Na}+$ ion adducts of the oligomer showing the homologous raw of $\mathrm{AA}$ and $\mathrm{BB}$ monomer additions in the TCC-polymer product. Blue colored bars represent the difference corresponding to AABB dimer segments). 
<smiles>O=C1C=CC(C2(c3cc(O)c(O)c(SC(CO)CSc4cc(C5(c6cc(=O)c(=O)c(=O)c6=O)c6ccccc6-c6ccccc65)cc(O)c4O)c3)c3ccccc3-c3ccccc32)=CC1=O</smiles>

Yield

GPC

$\mathrm{T}_{\mathrm{g}}$

TGA

${ }^{1} \mathrm{H}-\mathrm{NMR}(500 \mathrm{MHz})$ in $\mathrm{DMSO}_{-} \mathrm{d}_{6}$

FT-IR $\left[\mathrm{cm}^{-1}\right]$

$49 \%$

$\mathrm{M}_{w, a p p}=3.20 \mathrm{~kg} / \mathrm{mol}, \mathrm{Ð}=1.7, \mathrm{DP}(\mathrm{AA}+\mathrm{BB})=6$

$97^{\circ} \mathrm{C}$

$325^{\circ} \mathrm{C}$ (maximal degradation rate)

$\delta[\mathrm{ppm}] 9.63$ - 8.49 (m, br.; 4H; BQ ArOH), 8.05 - 6.26 (m, br.; 12H; BQ ArH), 5.20 - 4.78 (m, br.; 1H; DT aliphatic H), $3.32-2.756$ (m, br.; 4H; DT aliphatic H).

3343 (s), 3091 (m), 2995 (m), 2944 (m), 1770 (m), 1760 (m), 1697

(m), 1645 (m), 1585 (m), 1473 (m), 1445 (m), 1412 (m), 1357 (m9, 1242 (s), 1167 (m), 1118 (m), 1097 (m), 1061 (m), 1021 (m), 897 (w), $852(\mathrm{w}), 819(\mathrm{w}), 752(\mathrm{w}), 734(\mathrm{~m}), 658(\mathrm{w}), 619(\mathrm{w})$.

MALDI-TOF-MS calculated $(\mathrm{m} / \mathrm{z}):[\mathrm{M}(4 \mathrm{AA}+4 \mathrm{BB})+\mathrm{Na}]^{+}=2035$

found $(\mathrm{m} / \mathrm{z}):[\mathrm{M}(4 \mathrm{AA}+4 \mathrm{BB})+\mathrm{Na}]^{+}=2035$
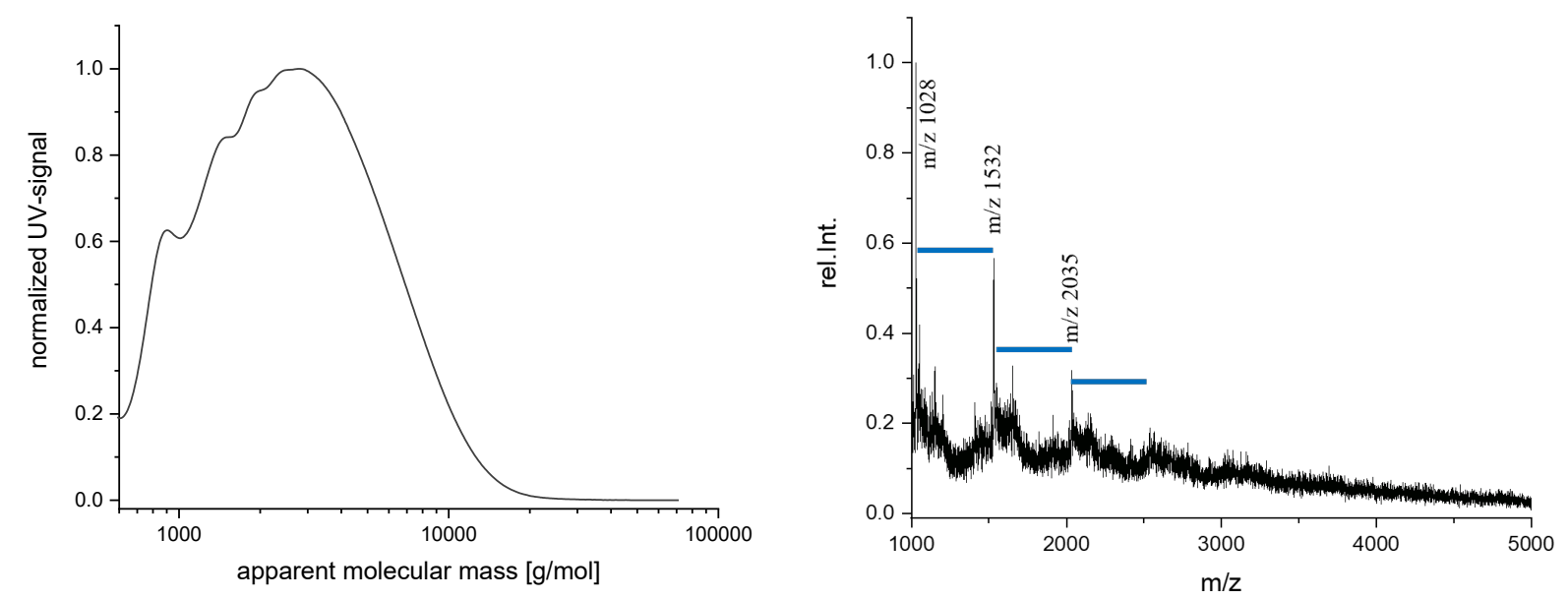

Figure S 52. p(BQFL-DMP) analysis. GPC chromatogram at $280 \mathrm{~nm}$ (left) and MALDI-TOF-MS spectrum (right, $\mathrm{m} / \mathrm{z}$ values were assigned to $\mathrm{Na}+$ ion adducts of the oligomer showing the homologous raw of $\mathrm{AA}$ and $\mathrm{BB}$ monomer additions in the TCC-polymer product. Blue colored bars represent the difference corresponding to AABB dimer segments. Despite optimization of matrix and sample preparation means, the TCC polymer shows a challenging desorption behavior, preventing to obtain well resolved MALDI-TOF-MS spectra). 
<smiles>O=C(CSc1cc(C2(C3=CC(=O)C(=O)C=C3)c3ccccc3-c3ccccc32)cc(O)c1O)OCCOC(=O)CSc1cc(C2(c3cc(=O)c(=O)c(=O)c3=O)c3ccccc3-c3ccccc32)cc(O)c1O</smiles>

Yield

GPC

$\mathrm{T}_{\mathrm{g}}$

TGA

${ }^{1} \mathrm{H}-\mathrm{NMR}(500 \mathrm{MHz})$ in $\mathrm{DMSO}_{-} \mathrm{d}_{6}$

FT-IR $\left[\mathrm{cm}^{-1}\right]$

$17 \%$

$\mathrm{M}_{w, \text { app }}=5.44 \mathrm{~kg} / \mathrm{mol}, \mathrm{Ð}=2.0, \mathrm{DP}(\mathrm{AA}+\mathrm{BB})=9$

$109{ }^{\circ} \mathrm{C}$

$367{ }^{\circ} \mathrm{C}$ (maximal degradation rate)

$\delta[\mathrm{ppm}] 9.64-8.63$ (m, br.; 4H; BQ ArOH), 8.01 - 6.23 (m, br.; 12H; BQ ArH), 4.27 - 3.41 (m, br.; 7H; DT aliphatic H.).

3372 (m), 3061 (m), 2995 (m), 1758 (m), 1729 (m), 1702 (s), 1656

(w), 1587 (m), 1475 (m), 1446 (m), 1411 (m), 1358 (m), 1489 (s),

1167 (s), 1120 (s), 1055 (m), 1024 (m), 974 (w), 893 (w), 853 (w),

$752(\mathrm{w}), 735(\mathrm{~m}), 697(\mathrm{w}), 656(\mathrm{w}), 619(\mathrm{w})$.

MALDI-TOF-MS calculated $(\mathrm{m} / \mathrm{z}):[\mathrm{M}(4 \mathrm{AA}+4 \mathrm{BB})+\mathrm{Na}]^{+}=2378.6$

found $(\mathrm{m} / \mathrm{z}):[\mathrm{M}(4 \mathrm{AA}+4 \mathrm{BB})+\mathrm{Na}]^{+}=2378.6$
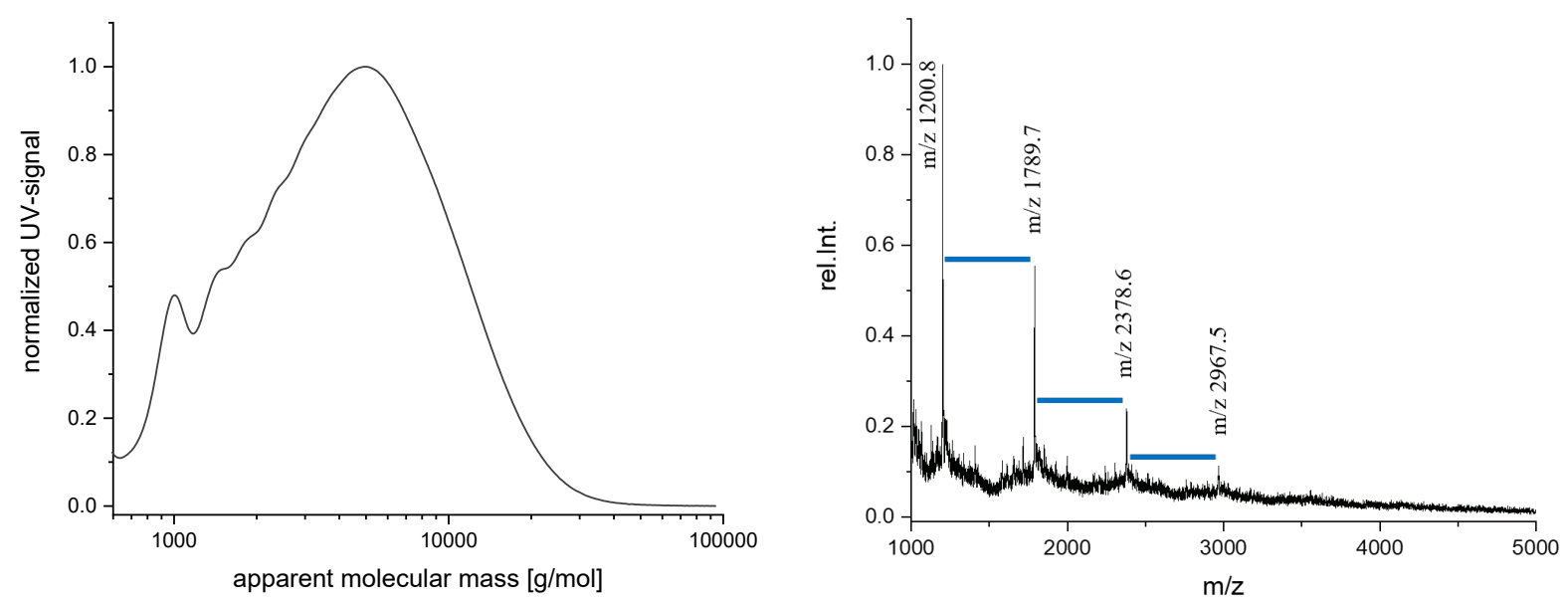

Figure S 53. p(BQFL-GDMA) analysis. GPC chromatogram at $280 \mathrm{~nm}$ (left) and MALDI-TOF-MS spectrum (right, $\mathrm{m} / \mathrm{z}$ values were assigned to $\mathrm{Na}+$ ion adducts of the oligomer showing the homologous raw of $\mathrm{AA}$ and $\mathrm{BB}$ monomer additions in the TCC-polymer product. Blue colored bars represent the difference corresponding to AABB dimer segments). 
<smiles>O=C(CCSc1cc(C2(C3=CC(=O)C(=O)C=C3)c3ccccc3-c3ccccc32)cc(O)c1O)OCCOC(=O)CCSc1cc(C2(c3cc(=O)c(=O)c(=O)c3=O)c3ccccc3-c3ccccc32)cc(O)c1O</smiles>
Yield
GPC
$\mathrm{T}_{\mathrm{g}}$
TGA
${ }^{1} \mathrm{H}-\mathrm{NMR}$ (500 MHz) in $\mathrm{DMSO}_{6} \mathrm{~d}_{6}$

FT-IR $\left[\mathrm{cm}^{-1}\right]$

$24 \%$

$\mathrm{M}_{w, \text { app }}=4.32 \mathrm{~kg} / \mathrm{mol}, \mathrm{Ð}=1.9, \mathrm{DP}(\mathrm{AA}+\mathrm{BB})=7$

no $\mathrm{T}_{\mathrm{g}}$ detected

$341{ }^{\circ} \mathrm{C}$ (maximal degradation rate)

$\delta[\mathrm{ppm}] 9.53$ - 8.46 (m, br.; 4H; BQ ArOH), 8.00 - 6.22 (m, br.; 12H; BQ ArH), 4.28 - 4.00 (m, br.; 1H; DT aliphatic H), 3.05 2.22 (m, br.; 10H; DT aliphatic H; integral includes signals of DMSO and NMP).

3372 (m), 3062 (w), 2995 (w), 2958 (w), 1732 (s), 1705 (s), 1588

(w), 1474 (m), 1444 (m), 1411 (m), 1357 (m), 1231 (s), 1184 (m), 1145 (s), $1058(\mathrm{w}), 1028(\mathrm{w}), 929(\mathrm{w}), 898(\mathrm{w}), 854(\mathrm{w}), 814(\mathrm{w})$, $752(\mathrm{w}), 737(\mathrm{~m}), 662(\mathrm{w}), 619(\mathrm{w})$.

MALDI-TOF-MS calculated $(\mathrm{m} / \mathrm{z}):[\mathrm{M}(4 \mathrm{AA}+4 \mathrm{BB})+\mathrm{Na}]^{+}=2491.5$

found $(\mathrm{m} / \mathrm{z}):[\mathrm{M}(4 \mathrm{AA}+4 \mathrm{BB})+\mathrm{Na}]^{+}=2491.6$
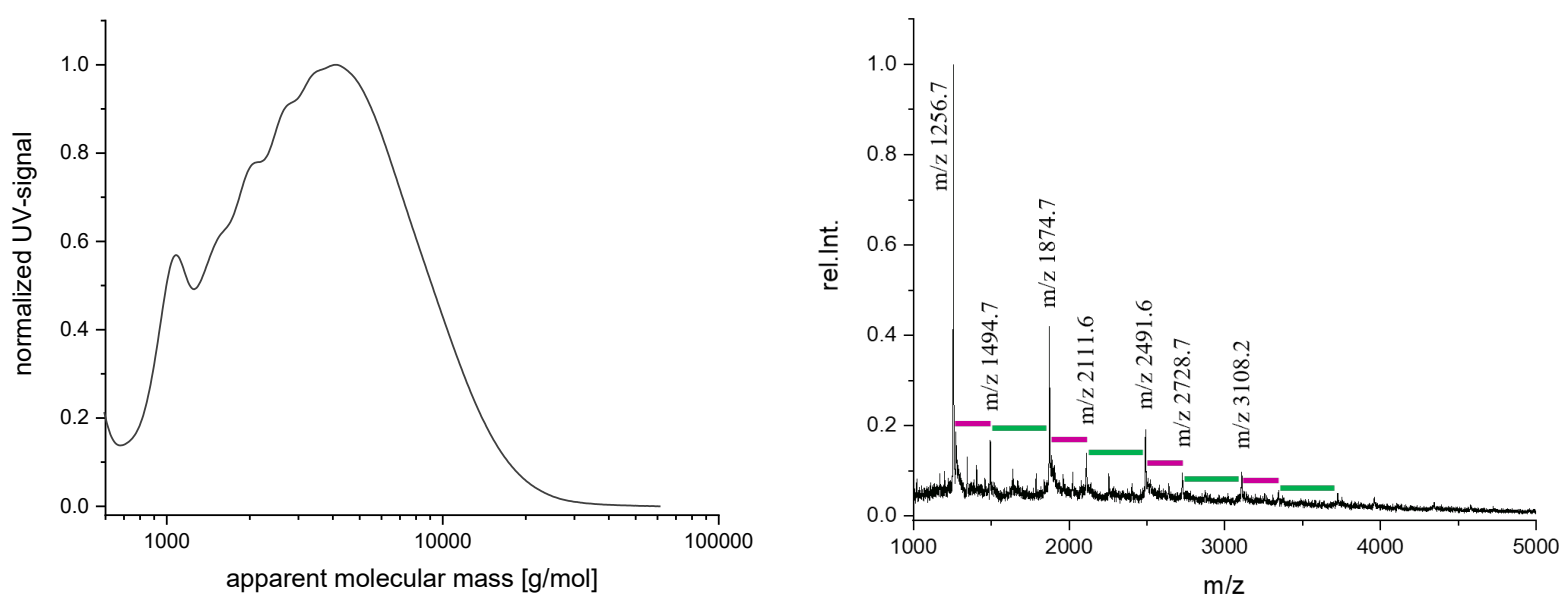

Figure S 54. p(BQFL-GDMP) analysis. GPC chromatogram at $280 \mathrm{~nm}$ (left) and MALDI-TOF-MS spectrum (right), $\mathrm{m} / \mathrm{z}$ values were assigned to $\mathrm{Na}+$ ion adducts of the oligomer showing the homologous raw of $\mathrm{AA}$ and $\mathrm{BB}$ monomer additions in the TCC-polymer product. Colored bars represent the $\mathrm{m} / \mathrm{z}$ differences corresponding to the repeating units, where green refers to biscatechols and purple to dithiols). 
<smiles>O=C1C=CC(C2(c3cc(O)c(O)c(SCCCCSc4cc(C5(C6=CC(=O)C(=O)C=C6)c6ccccc6-c6ccccc65)cc(O)c4O)c3)c3ccccc3-c3ccccc32)=CC1=O</smiles>

Yield

GPC

$\mathrm{T}_{\mathrm{g}}$

TGA

${ }^{1} \mathrm{H}-\mathrm{NMR}(500 \mathrm{MHz})$ in $\mathrm{DMSO}_{-} \mathrm{d}_{6}$

FT-IR $\left[\mathrm{cm}^{-1}\right]$

$59 \%$

$\mathrm{M}_{w, \text { app }}=2.73 \mathrm{~kg} / \mathrm{mol}, \mathrm{Ð}=1.8, \mathrm{DP}(\mathrm{AA}+\mathrm{BB})=5$

$139{ }^{\circ} \mathrm{C}$

$346{ }^{\circ} \mathrm{C}$ (maximal degradation rate)

$\delta[\mathrm{ppm}] 9.66$ - 8.20 (m, br.; 4H; BQ ArOH), 7.99 - 6.11 (m, br.; 12H; BQ ArH), $3.13-1.88$ (m, br.; 6H; DT aliphatic H, includes the signal of DMSO), 1.84 - 0.59 (m, br.; 4H; DT aliphatic H).

3365 (m), 3061 (m), 3003 (m), 2929 (m), 2854 (m), 1771 (w), 1758

(w), 1697 (m), 1645 (w), 1589 (m), 1472 (m), 1447 (m), 1411 (m), 1352 (m), 1319 (m), 1251 (s), 1232 (s), 1165 (m), 1119 (m), 1094 (m), $1024(\mathrm{w}), 907(\mathrm{w}), 851(\mathrm{w}), 811(\mathrm{w}), 750(\mathrm{w}), 735(\mathrm{~m}), 701(\mathrm{w})$, $655(\mathrm{w}), 618(\mathrm{w})$.

MALDI-TOF-MS calculated $(\mathrm{m} / \mathrm{z}):[\mathrm{M}(4 \mathrm{AA}+4 \mathrm{BB})+\mathrm{Na}]^{+}=2027$ found $(\mathrm{m} / \mathrm{z}):[\mathrm{M}(4 \mathrm{AA}+4 \mathrm{BB})+\mathrm{Na}]^{+}=2027$
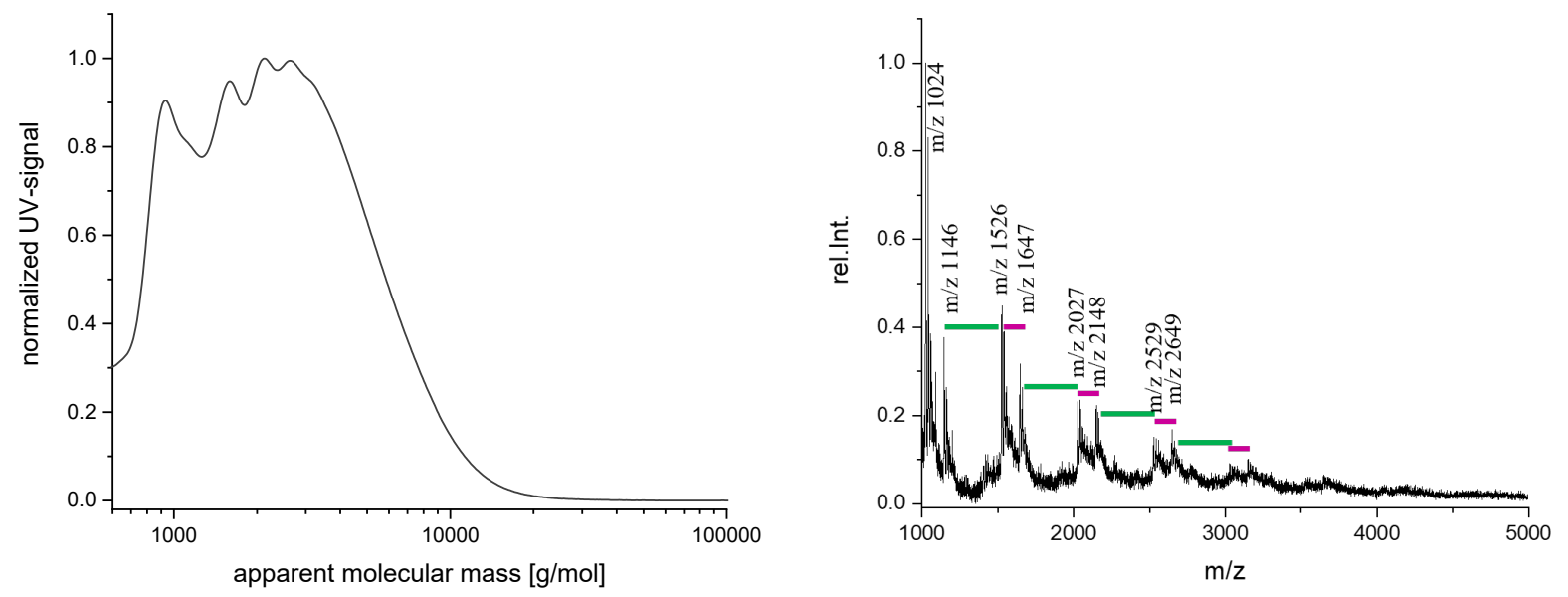

Figure S 55. p(BQFL-BDT) analysis. GPC chromatogram at $280 \mathrm{~nm}$ (left) and MALDI-TOF-MS spectrum (right, $\mathrm{m} / \mathrm{z}$ values were assigned to $\mathrm{Na}+$ ion adducts of the oligomer showing the homologous raw of $\mathrm{AA}$ and $\mathrm{BB}$ monomer additions in the TCC-polymer product. Colored bars represent the $\mathrm{m} / \mathrm{z}$ differences corresponding to the repeating units, where green refers to biscatechols and purple to dithiols. Despite optimization of matrix and sample preparation means, the TCC polymer shows a challenging desorption behavior, preventing to obtain well resolved MALDI-TOF-MS spectra). 
<smiles>O=C1C=CC(C2(c3cc(O)c(O)c(SCCCCCCSc4cc(C5(C6=CC(O)C(O)C=C6)c6ccccc6-c6ccccc65)cc(O)c4O)c3)c3ccccc3-c3ccccc32)=CC1=O</smiles>

Yield

GPC

$\mathrm{T}_{\mathrm{g}}$

TGA

${ }^{1} \mathrm{H}-\mathrm{NMR}(500 \mathrm{MHz})$ in $\mathrm{DMSO}_{-} \mathrm{d}_{6}$

FT-IR $\left[\mathrm{cm}^{-1}\right]$

$48 \%$

$\mathrm{M}_{w, \text { app }}=4.56 \mathrm{~kg} / \mathrm{mol}, \mathrm{Ð}=1.8, \mathrm{DP}(\mathrm{AA}+\mathrm{BB})=9$

$120{ }^{\circ} \mathrm{C}$

$356{ }^{\circ} \mathrm{C}$ (maximal degradation rate)

$\delta[\mathrm{ppm}] 9.51$ - 8.36 (m, br.; 5H; BQ ArOH), 8.01 - 6.22 (m, br.; 12H; BQ ArH), 2.82 - 1.92 (m, br.; 8H; DT aliphatic H; integral includes signal of DMSO), 1.66 - 0.44 (m, br.; 11H; DT aliphatic H).

3387 (s), 2995 (m), 2929 (s), 2855 (m), 1771 (m), 1701 (m), 1653

(w), 1592 (w), 1477 (m), 1448 (m), 1413 (m), 1359 (m), 1320 (m), 1240 (s), 1180 (m), $1116(\mathrm{~m}), 1058(\mathrm{w}), 927$ (w), $899(\mathrm{w}), 806(\mathrm{w})$, $752(\mathrm{w}), 736(\mathrm{~m}), 659(\mathrm{w})$.

MALDI-TOF-MS calculated $(\mathrm{m} / \mathrm{z}):[\mathrm{M}(4 \mathrm{AA}+4 \mathrm{BB})+\mathrm{Na}]^{+}=2138$

found $(\mathrm{m} / \mathrm{z}):[\mathrm{M}(4 \mathrm{AA}+4 \mathrm{BB})+\mathrm{Na}]^{+}=2138$
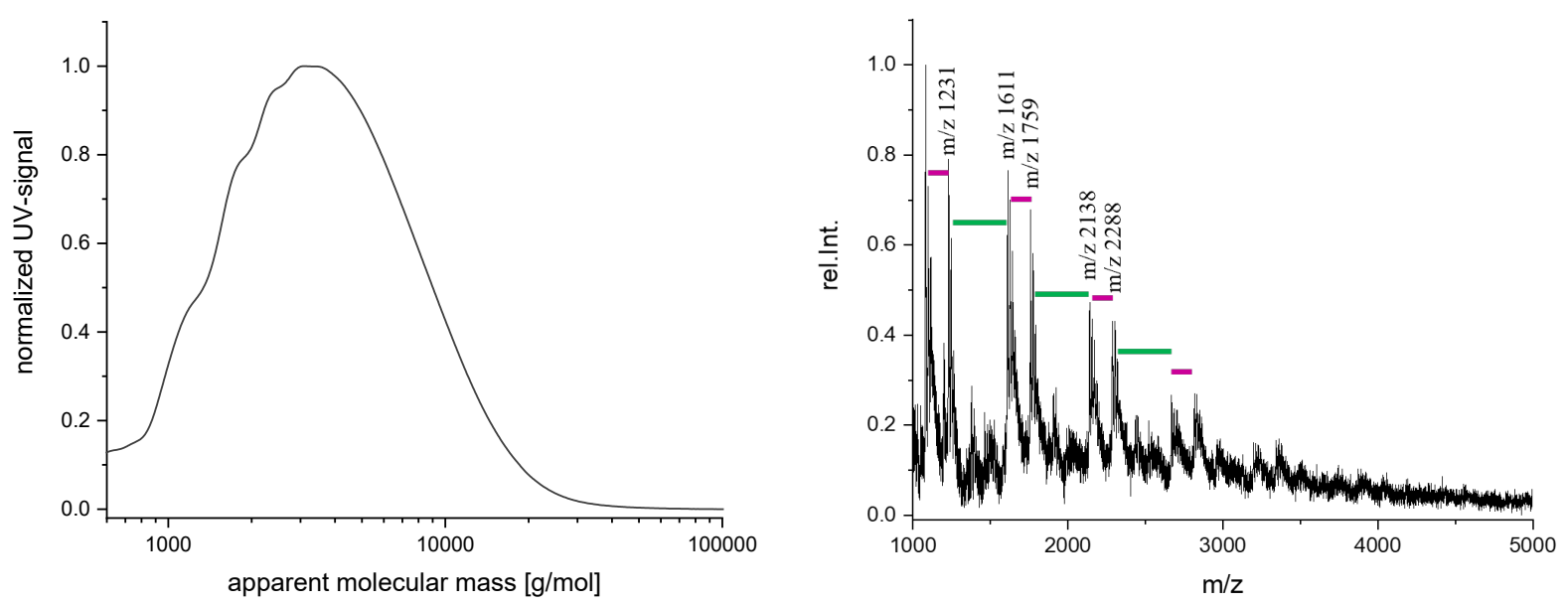

Figure S 56. p(BQFL-HDT) analysis. GPC chromatogram at $280 \mathrm{~nm}$ (left) and MALDI-TOF-MS spectrum (right, $\mathrm{m} / \mathrm{z}$ values were assigned to $\mathrm{Na}+$ ion adducts of the oligomer showing the homologous raw of $\mathrm{AA}$ and $\mathrm{BB}$ monomer additions in the TCC-polymer product. Colored bars represent the $\mathrm{m} / \mathrm{z}$ differences corresponding to the repeating units, where green refers to biscatechols and purple to dithiols. Despite optimization of matrix and sample preparation means, the TCC polymer shows a challenging desorption behavior, preventing to obtain well resolved MALDI-TOF-MS spectra). 


\section{Experiments}

\subsection{Time resolved investigation of MIPoly}

Polymerization was carried out for $15 \mathrm{~min}$ at room temperature. For this the bisquinone was solved in NMP (22.6 g/L), the solution was stirred, and one equivalent of the respective dithiol was added. Samples were withdrawn from the reaction mixture at different time points. Therefore, $40 \mu \mathrm{L}$ reaction mixture were diluted in $3 \mathrm{~mL}$ THF, filtrated, and analyzed by GPC. Results are shown in Figure S 57 to Figure S 62.
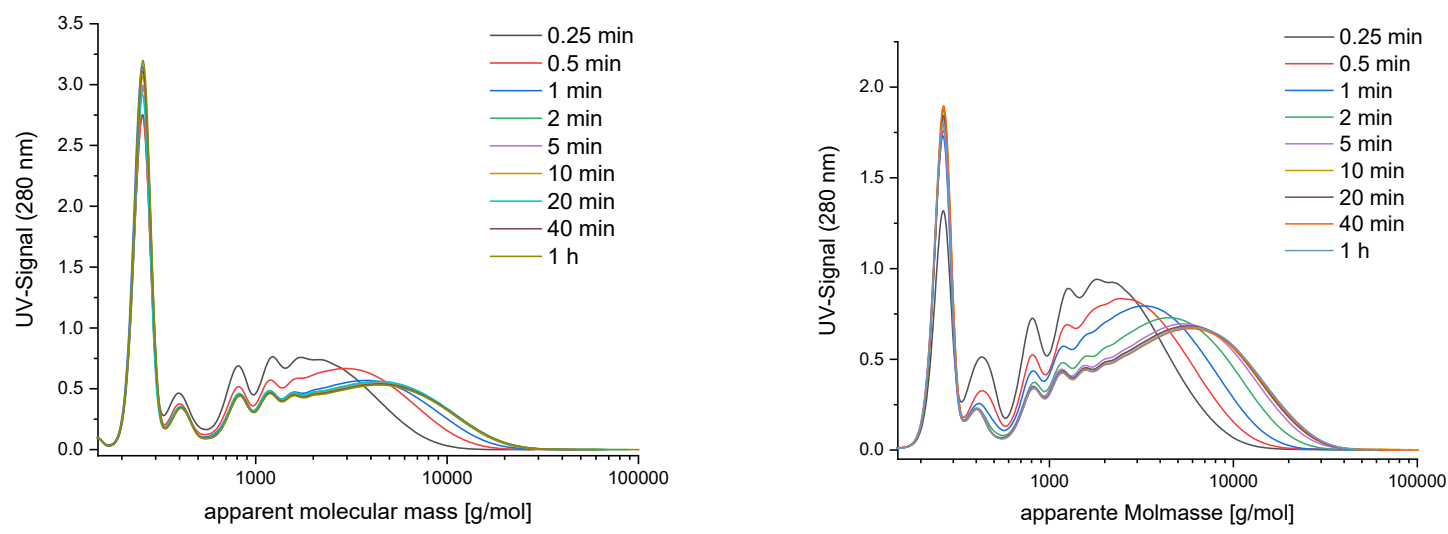

Figure S 57. GPC chromatograms of the time resolved investigation of $\mathrm{p}$ (BQA-EDET) (left) and $\mathrm{p}$ (BQA-BMT) (right). The first detected signal is assigned to the BHT-standard.
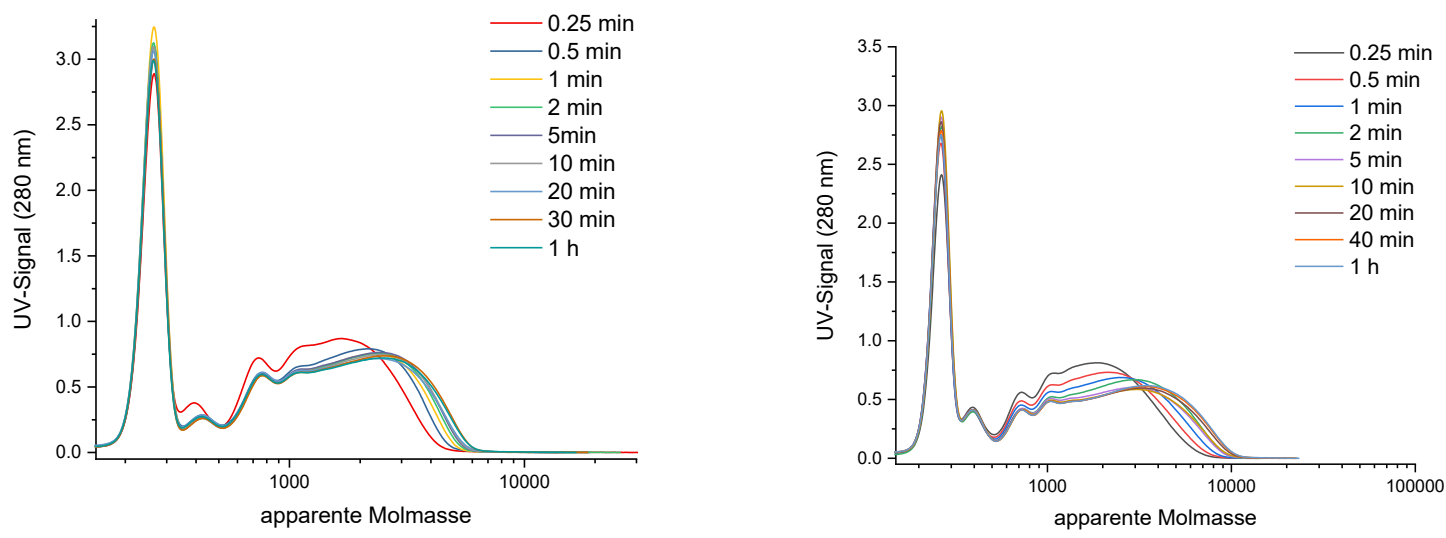

Figure S 58. GPC chromatograms of the time resolved investigation of p(BQA-DTT) (left) and p(BQA-DMP) (right). The first detected signal is assigned to the BHT-standard. 

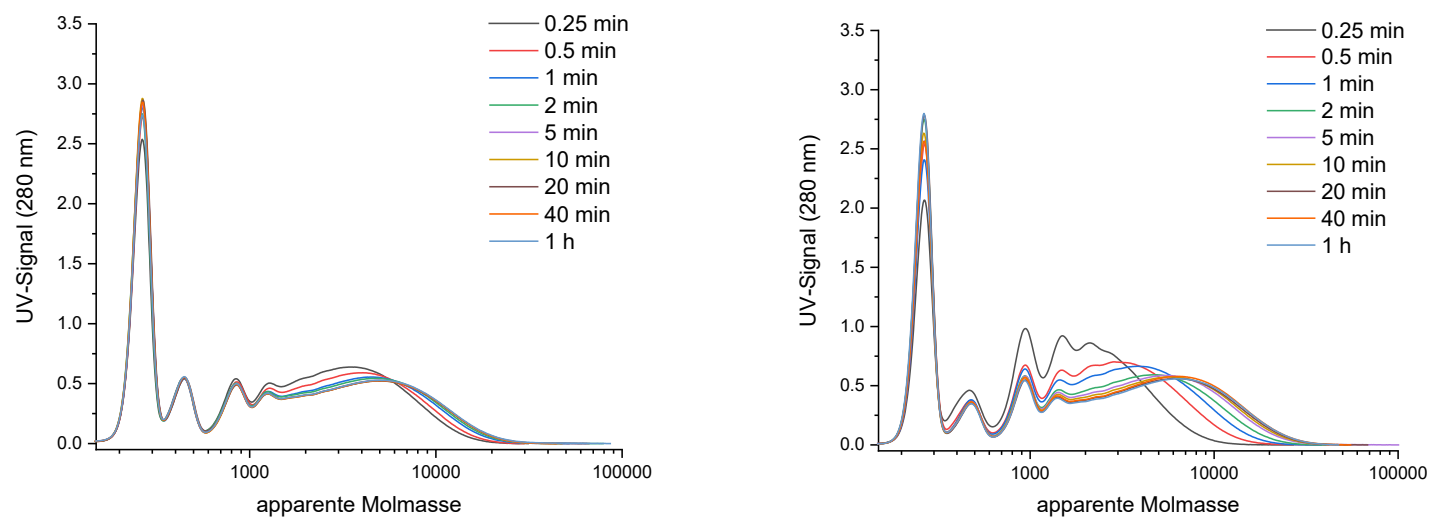

Figure S 59. GPC chromatograms of the time resolved investigation of p(BQA-GDMA) (left) and p(BQA-GDMP) (right). The first detected signal is assigned to the BHT-standard.
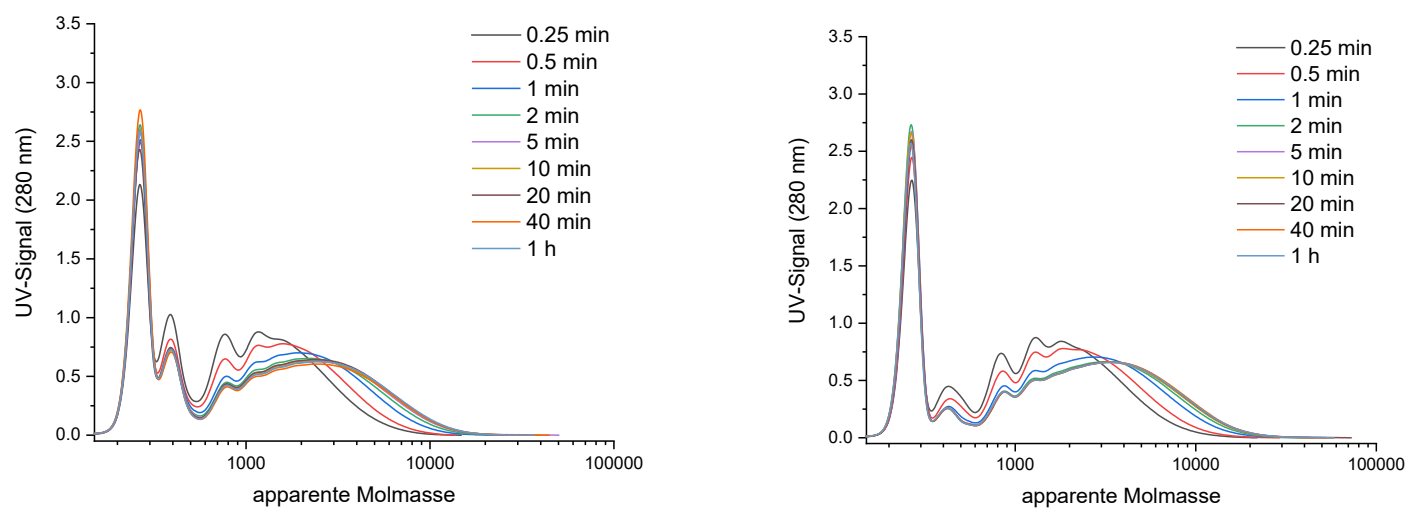

Figure S 60. GPC chromatograms of the time resolved investigation of p(BQA-BDT) (left) and p(BQA-HDT) (right). The first detected signal is assigned to the BHT-standard.
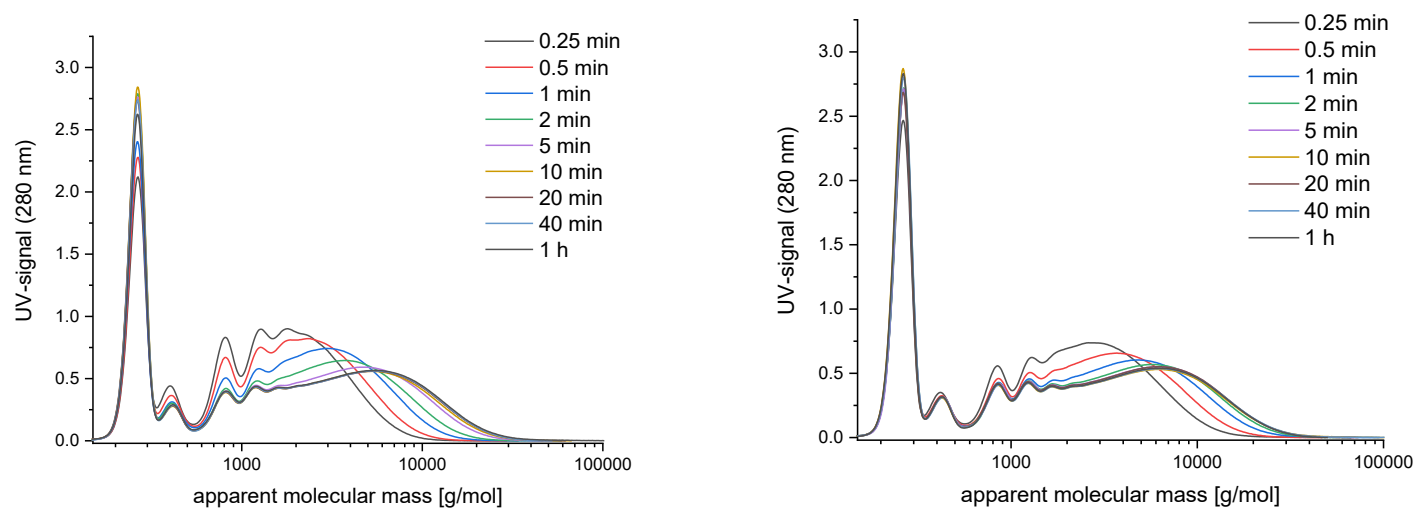

Figure S 61. GPC chromatograms of the time resolved investigation of p(BQZ-EDET) (left) and p(BQB-EDET) (right). The first detected signal is assigned to the BHT-standard. 

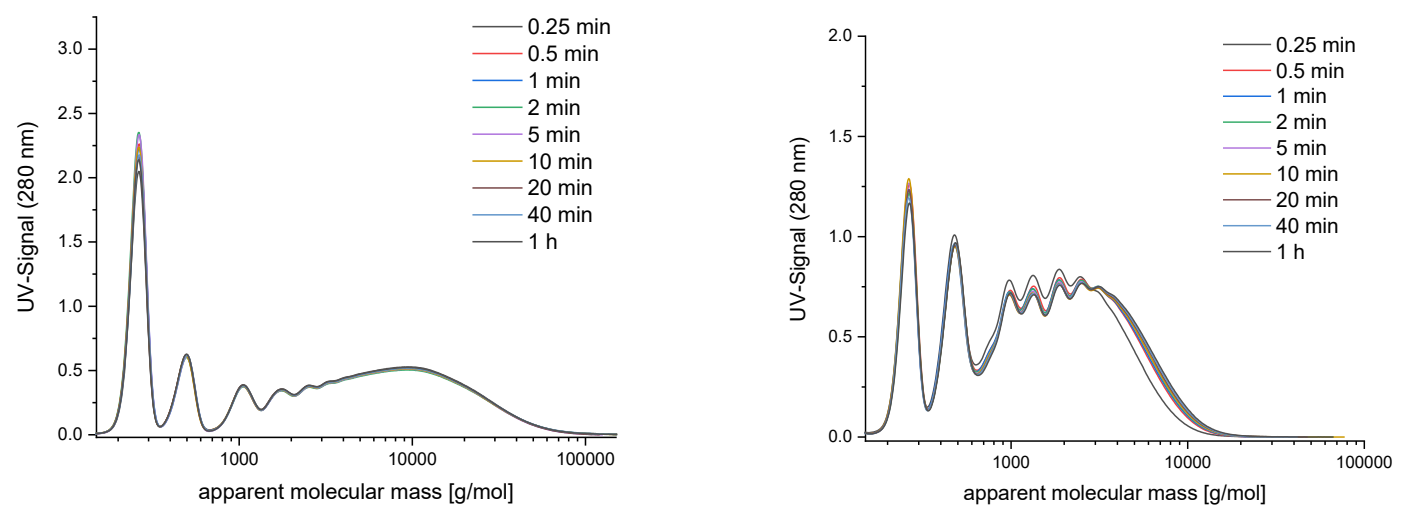

Figure S 62. GPC chromatograms of the time resolved investigation of $\mathrm{p}$ (BQM-EDET) (left) and $\mathrm{p}$ (BQFL-EDET) (right). The first detected signal is assigned to the BHT-standard.

\subsection{Calculation of the proportion of low molecular weight products}

Calculation of lower molecular weight proportions of up to $(\mathrm{AA}+\mathrm{BB})_{3}$ repeats was calculated based on the GPC chromatograms after 20 min received from the time resolved investigation of MIPoly of a representative set of monomer combinations (SI section 6.1). Therefore, the area of low molecular weight products (calculated from the internal BHT standard up to the apparent molecular mass corresponding to the molecular mass of (AA-BB $)_{3}$ repeats of each monomer combination) was related to the area of the whole chromatogram (calculated from the internal BHT standard to the end of the chromatogram). This is exemplarily shown in Figure S 63 and the results for the representative set of monomer combinations is shown in Table S 4 .
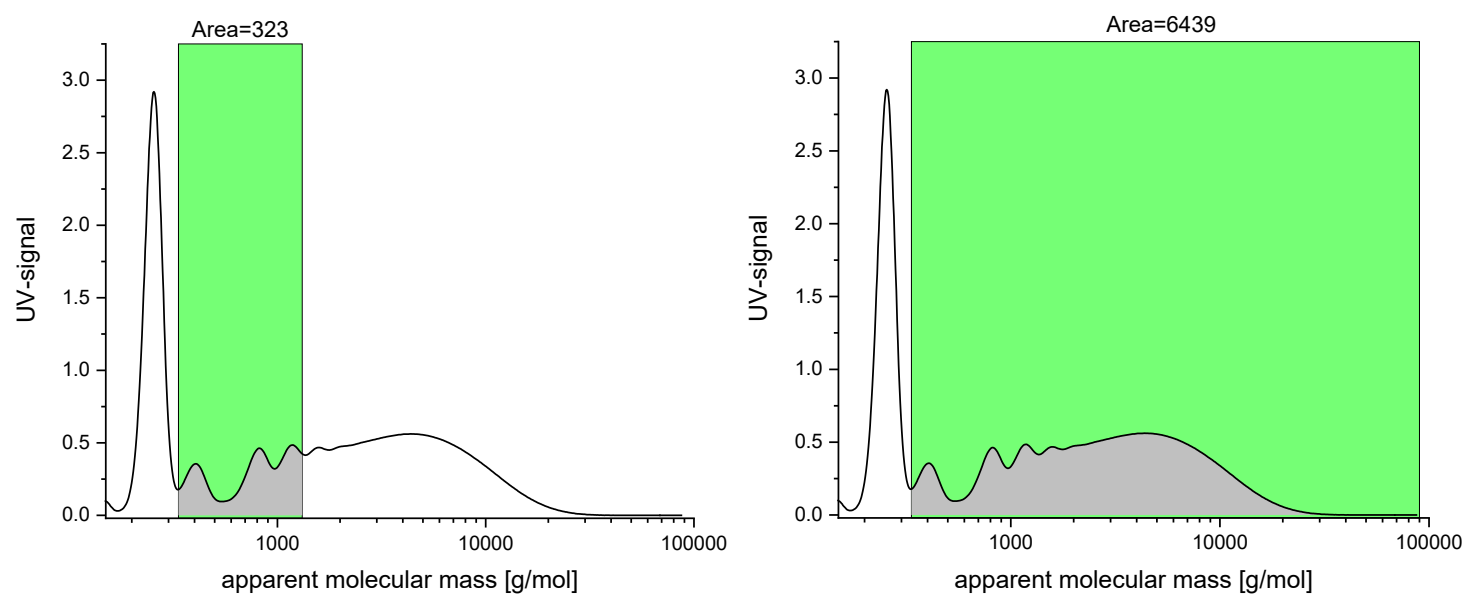

Figure S 63. Exemplarily integration of the area of the chromatograms of p(BQA-EDET) between 335 and 1316 $\mathrm{g} / \mathrm{mol}$ (left) as well as from 335 and $90000 \mathrm{~g} / \mathrm{mol}$ (right) (Signal at apparent $260 \mathrm{~g} / \mathrm{mol}$ corresponds to the internal standard). 


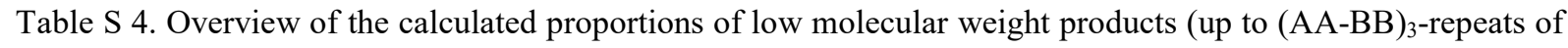
each monomer combination).

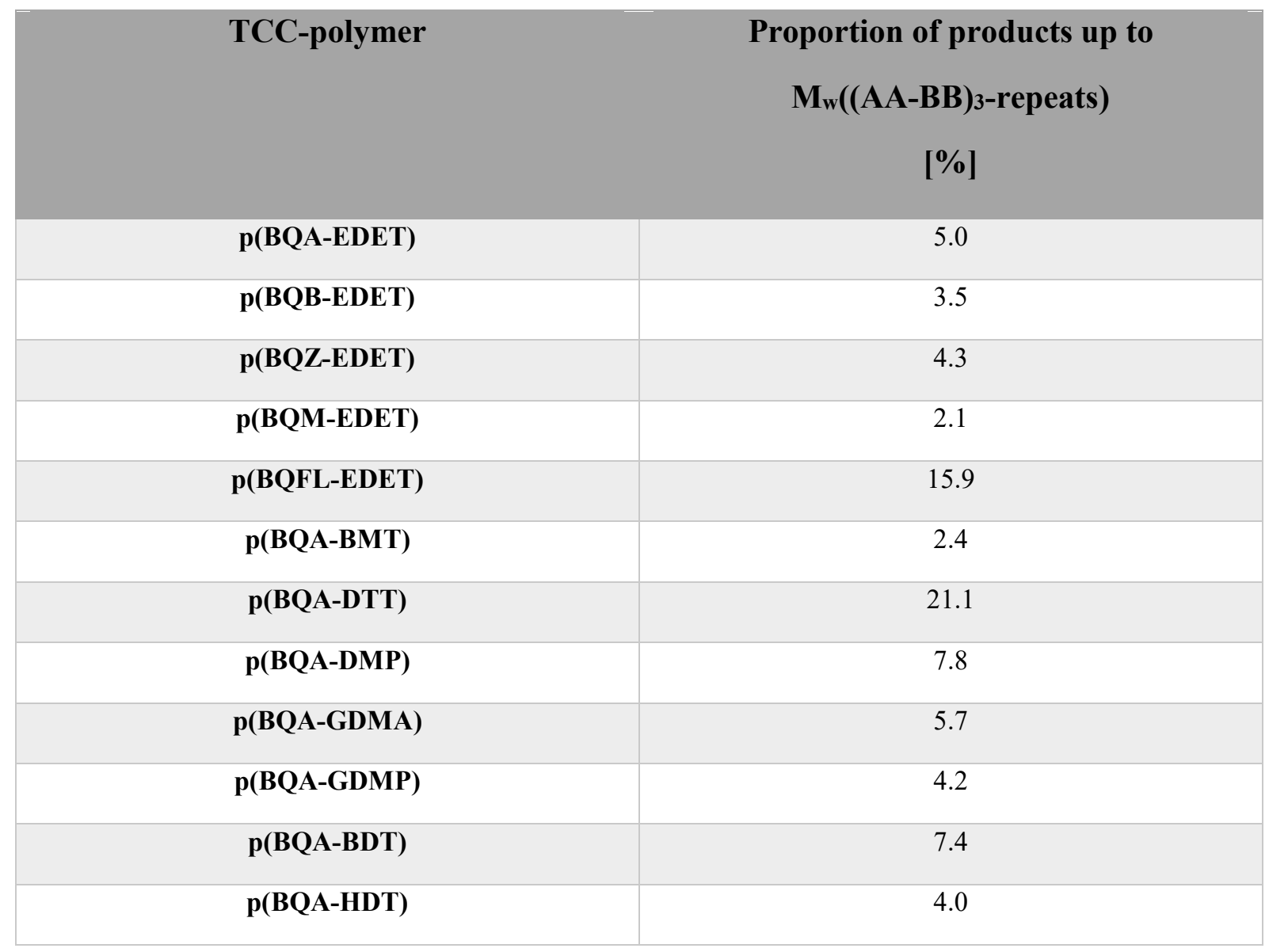


6.3 Lap shear test of TCC-polymer matrix under dry conditions

Lap shear tests were carried out according to the procedure in section 3.2 and 3.4.

Table S 5. Adhesive strength values for lap shear tests of TCC-polymer adhesive tests under dry conditions.

\begin{tabular}{|c|c|c|c|c|}
\hline BB & \multicolumn{4}{|c|}{$\begin{array}{l}\text { adhesive strength on aluminum [MPa] } \\
\text { adhesive strength on poly(propylene) [MPa] }\end{array}$} \\
\hline EDET & $\begin{array}{l}1.80 \pm 0.48 \\
0.87 \pm 0.28\end{array}$ & $\begin{array}{l}1.10 \pm 0.34 \\
0.94 \pm 0.10\end{array}$ & $\begin{array}{l}1.77 \pm 0.73 \\
0.75 \pm 0.04\end{array}$ & $\begin{array}{l}1.77 \pm 0.39 \\
1.19 \pm 0.22\end{array}$ \\
\hline BMT & $\begin{array}{l}0.74 \pm 0.22 \\
0.31 \pm 0.17\end{array}$ & $\begin{array}{l}0.97 \pm 0.19 \\
0.41 \pm 0.16\end{array}$ & --- & $\begin{array}{l}1.40 \pm 0.20 \\
0.96 \pm 0.26\end{array}$ \\
\hline DMP & $\begin{array}{l}0.96 \pm 0.45 \\
0.49 \pm 0.11\end{array}$ & $\begin{array}{l}0.61 \pm 0.31 \\
0.35 \pm 0.14\end{array}$ & --- & $\begin{array}{l}1.47 \pm 0.73 \\
0.72 \pm 0.31\end{array}$ \\
\hline GDMA & $\begin{array}{l}0.71 \pm 0.30 \\
0.60 \pm 0.07\end{array}$ & --- & --- & --- \\
\hline GDMP & $\begin{array}{l}1.80 \pm 0.48 \\
0.80 \pm 0.09\end{array}$ & $\begin{array}{l}0.43 \pm 0.13 \\
1.18 \pm 0.21\end{array}$ & --- & $\begin{array}{l}2.40 \pm 0.39 \\
1.12 \pm 0.12\end{array}$ \\
\hline BDT & $\begin{array}{l}0.51 \pm 0.10 \\
0.73 \pm 0.12\end{array}$ & --- & --- & --- \\
\hline HDT & $\begin{array}{l}0.85 \pm 0.21 \\
1.01 \pm 0.14\end{array}$ & $\begin{array}{l}0.88 \pm 0.40 \\
1.02 \pm 0.16\end{array}$ & --- & $\begin{array}{l}1.25 \pm 0.39 \\
1.26 \pm 0.16\end{array}$ \\
\hline
\end{tabular}


Table S 6. Force-extension curves of lap shear tests of TCC-polymers.

\section{BQA-family Aluminum}

$\mathrm{p}(\mathrm{BQA}-\mathrm{EDET})$

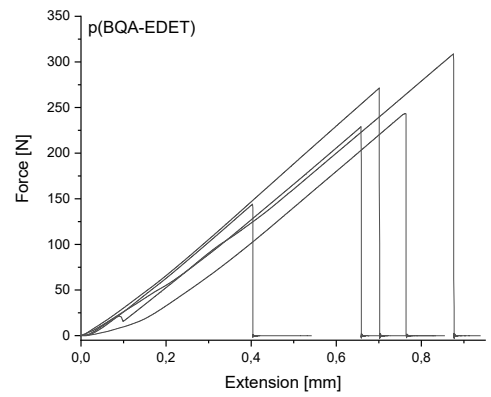

$\mathrm{p}(\mathrm{BQA}-\mathrm{BMT})$

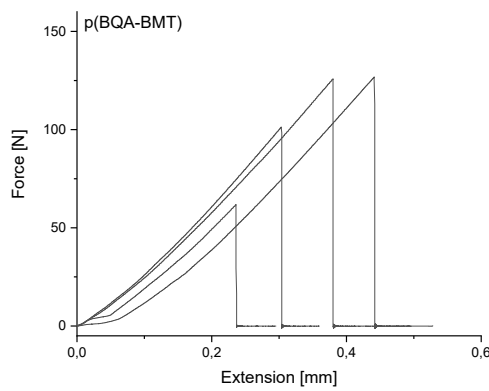

p(BQA-DMP)

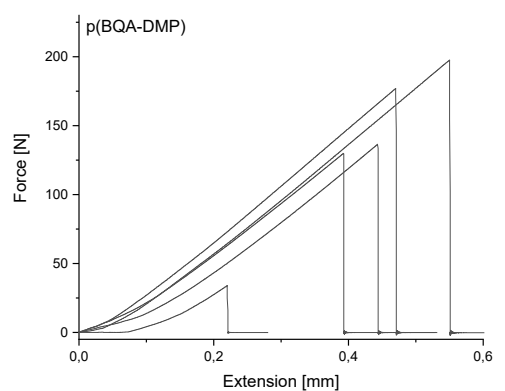

p(BQA-GDMA)

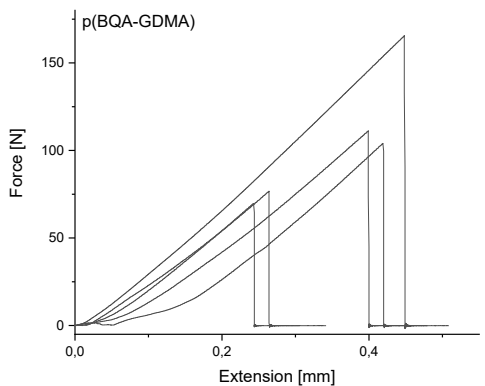

\section{Poly(propylene)}
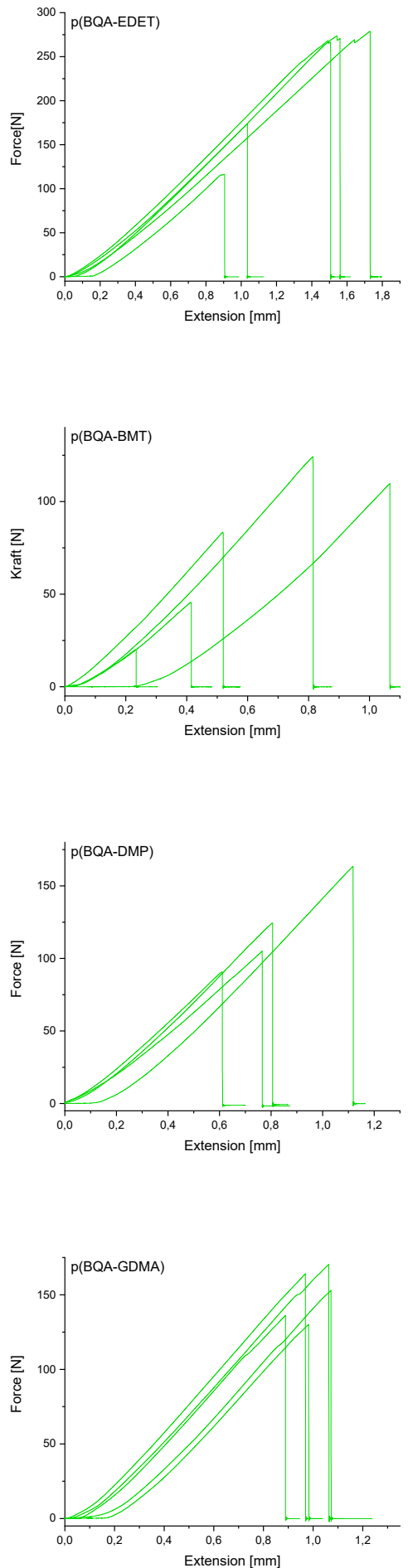
$\mathrm{p}$ (BQA-GDMP)
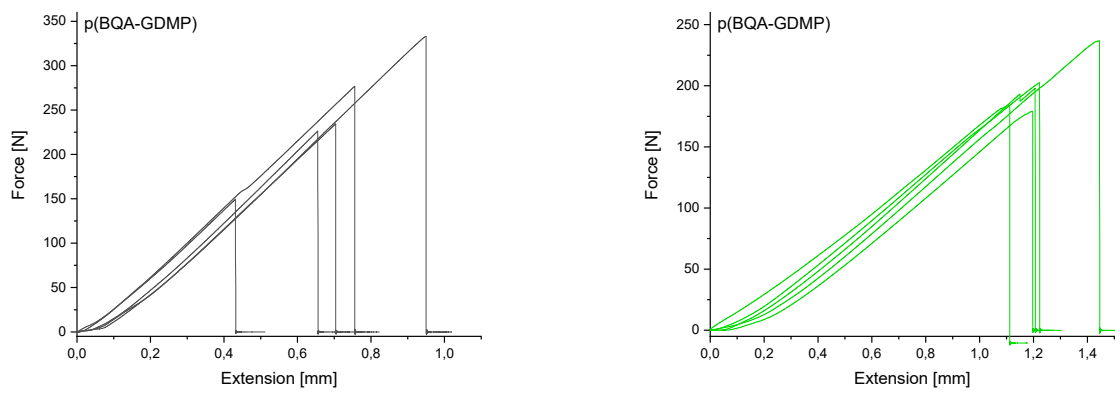

$\mathrm{p}(\mathrm{BQA}-\mathrm{BDT})$
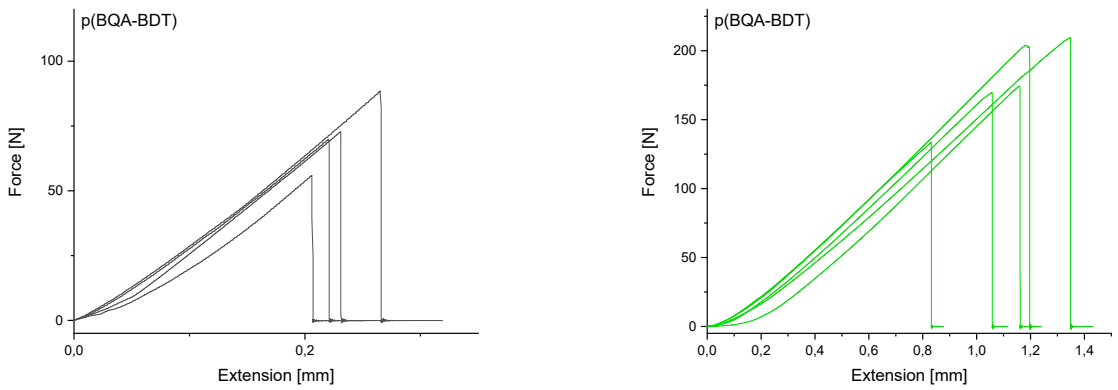

p(BQA-HDT)
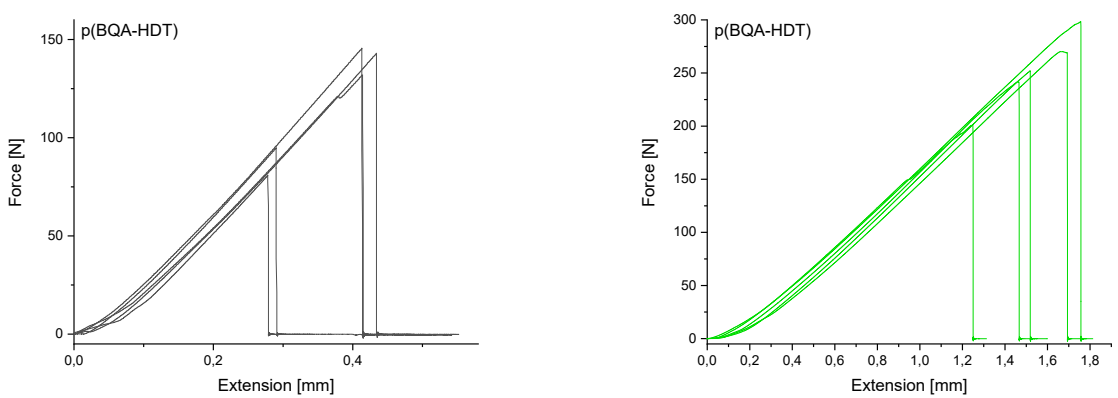
BQZ-family

Aluminum

p(BQZ-EDET)

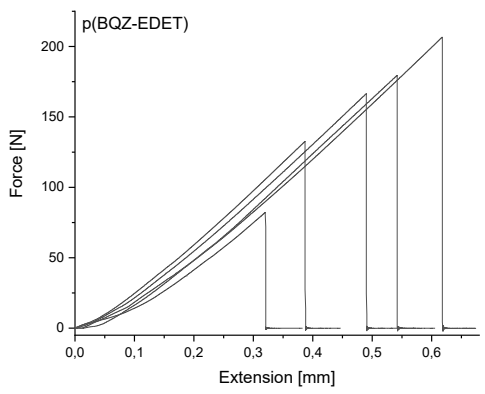

$\mathrm{p}(\mathrm{BQZ}-\mathrm{BMT})$

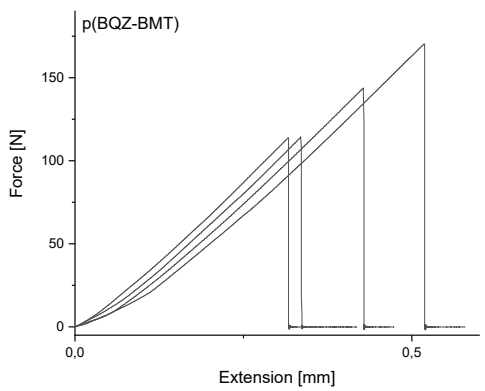

$\mathrm{p}(\mathrm{BQZ}-\mathrm{DMP})$

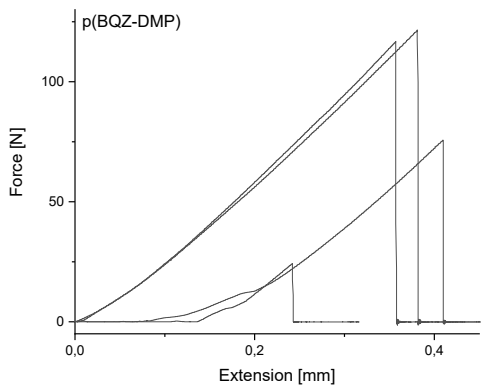

p(BQZ-GDMP)

Outliers are shown in dashed lines.

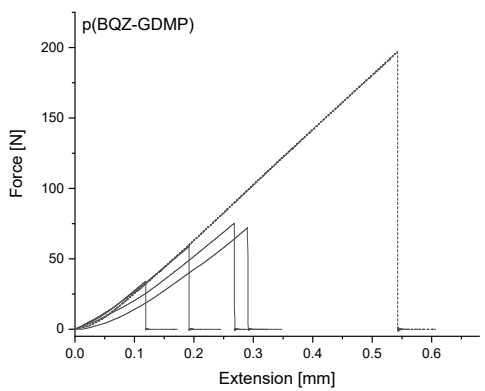

Poly(propylene)
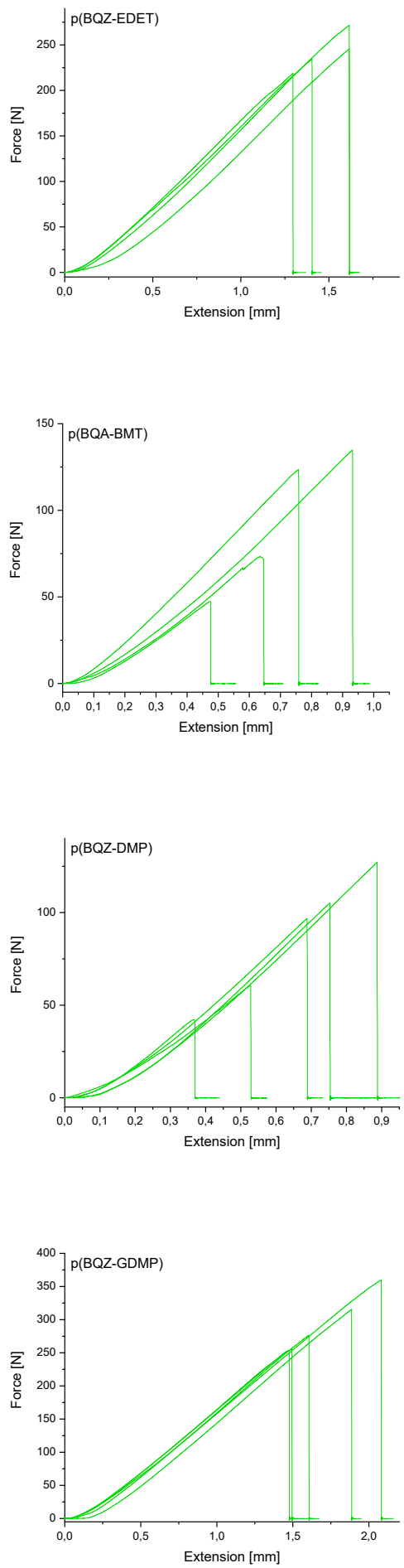
p(BQZ-HDT)
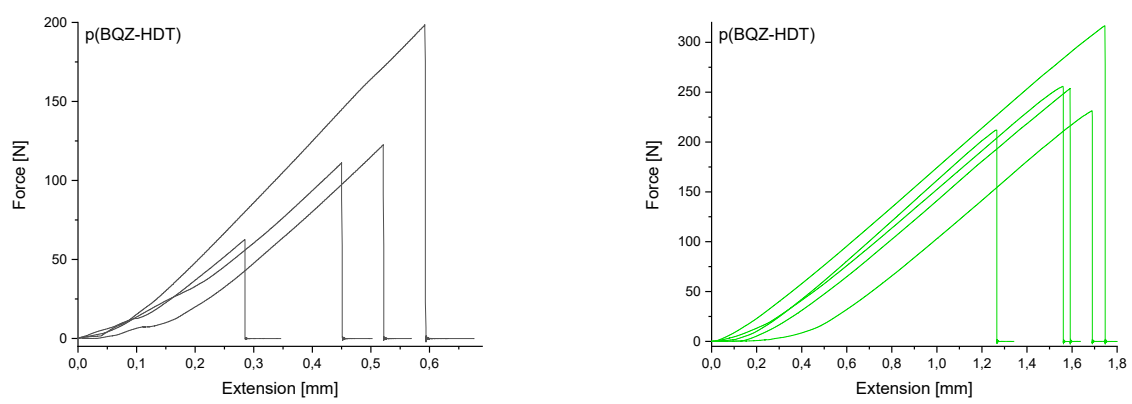

BQB-family

Aluminum

$\mathrm{p}(\mathrm{BQB}-\mathrm{EDET})$

Outliers are shown in dashed lines.

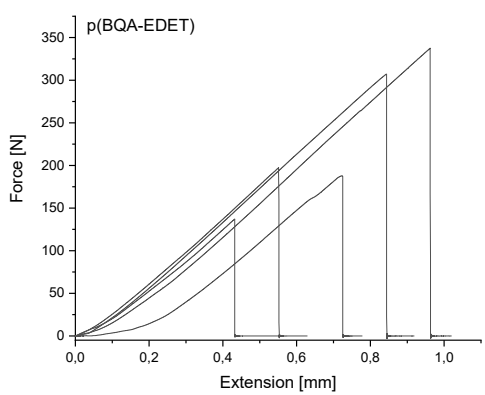

BQM-family

Aluminum

p(BQM-EDET)

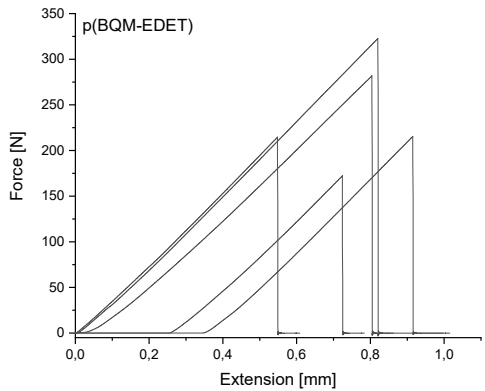

$\mathrm{p}(\mathrm{BQM}-\mathrm{BMT})$

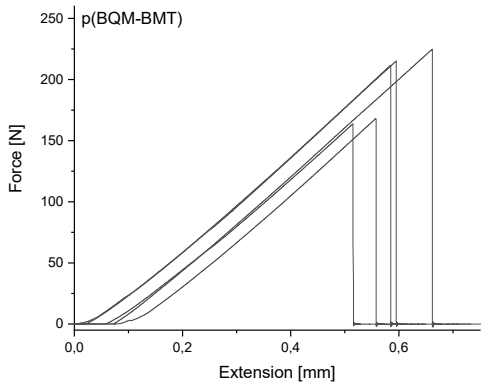

Poly(propylene)

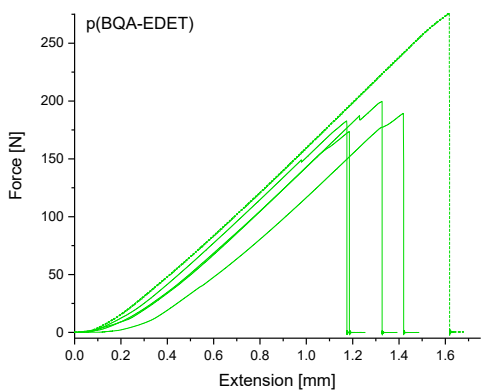

Poly(propylene)
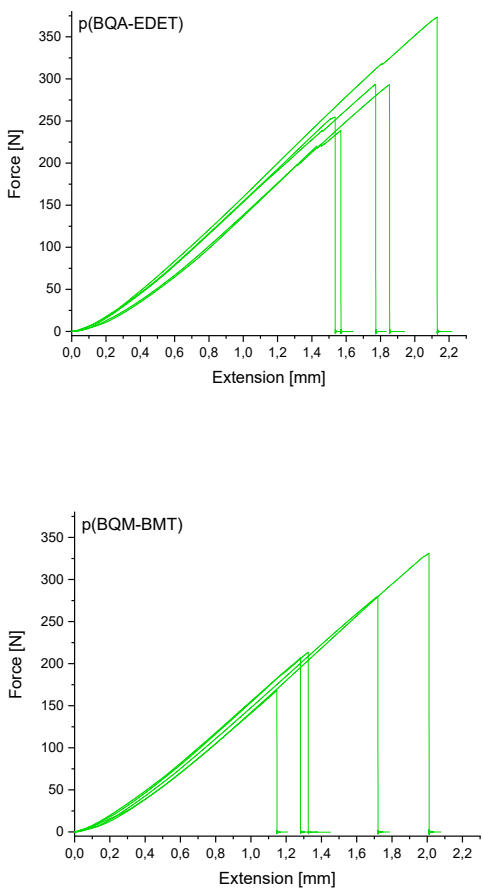
p(BQM-DMP)
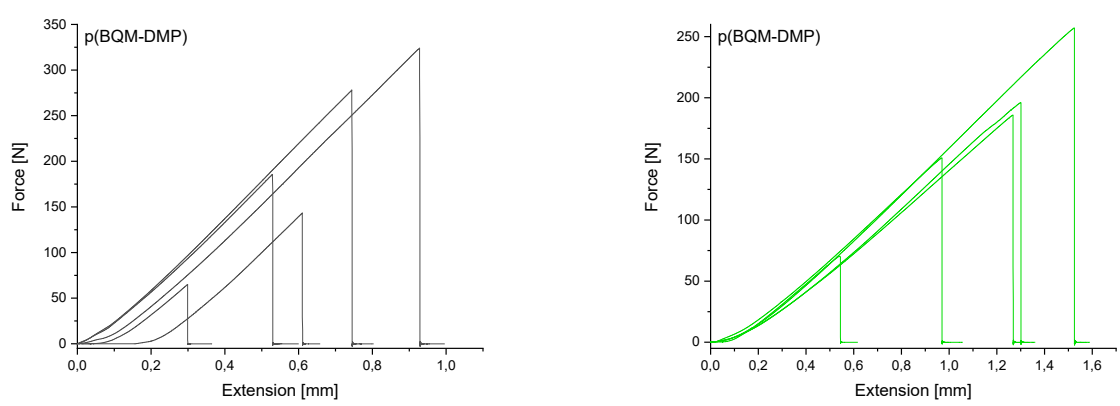

$p(B Q M-G D M P)$
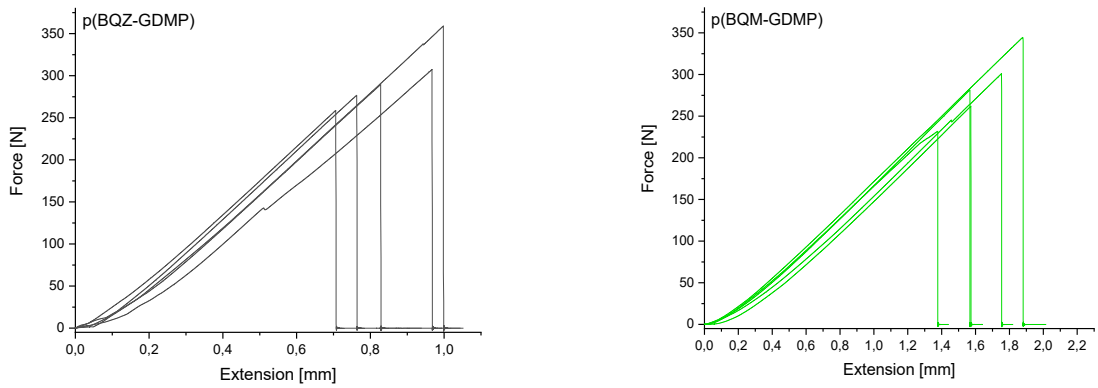

p(BQM-HDT)
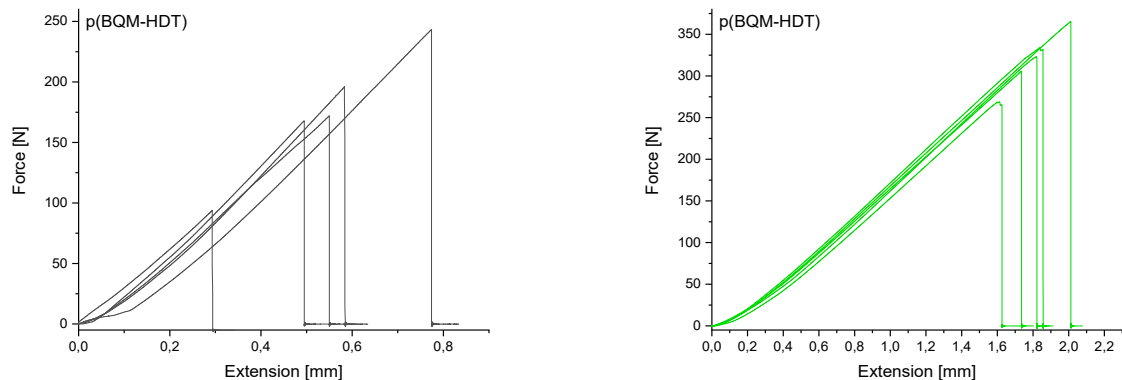
6.4 Exemplary pictures and SEM analysis of the test specimen surfaces after adhesive testing of TCC-polymer matrix

SEM analysis of marked area was carried out. Therefore, the surface of the polypropylene samples had to be scratched in order to enable recording. The scale bar corresponds to $500 \mu \mathrm{m}$ for the aluminum samples and $1 \mathrm{~mm}$ for the polypropylene samples. In addition, the respective adhesive surfaces were also examined using EDX to be able to detect the polymer based on the sulfur it contained.

The combination of both techniques confirmed that predominantly cohesive fractures occur on aluminum and predominantly adhesive fractures on PP.

The images and results obtained for bonding aluminum are listed below:

- uncoated aluminum:

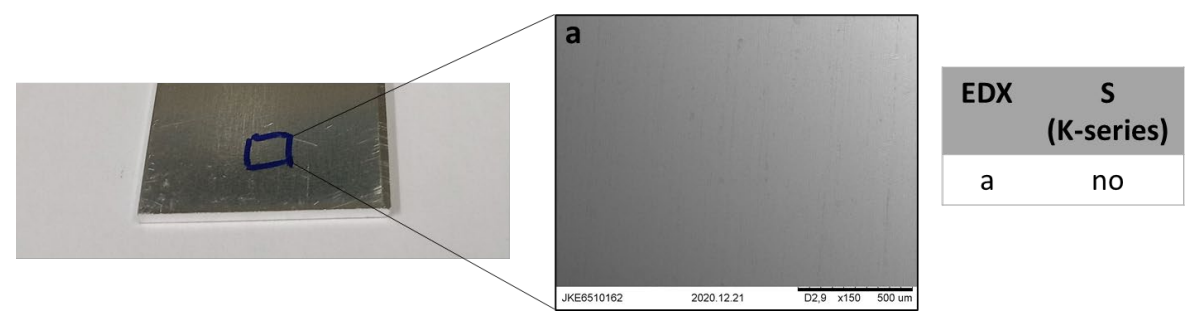

- $p(B Q A-E D E T)$ :
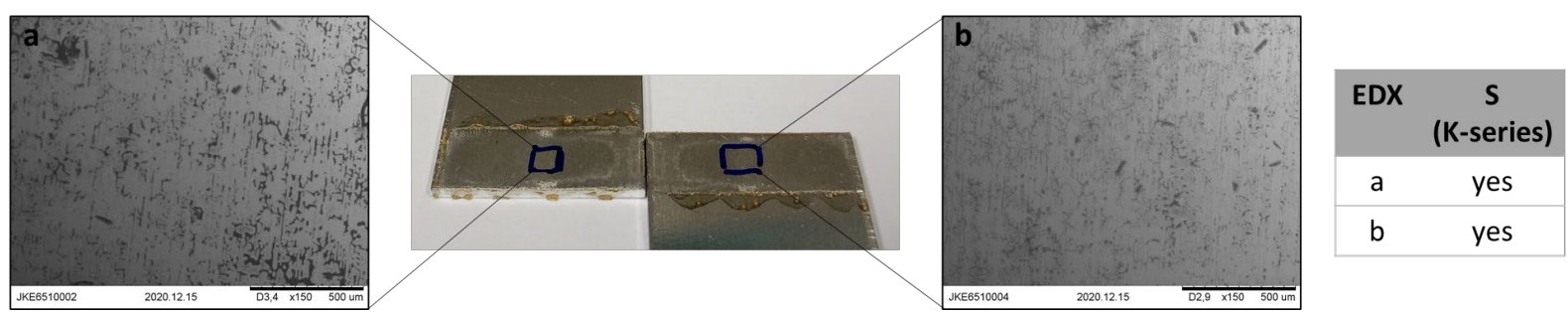

- $\quad \mathrm{p}(\mathrm{BQA}-\mathrm{BMT})$ :
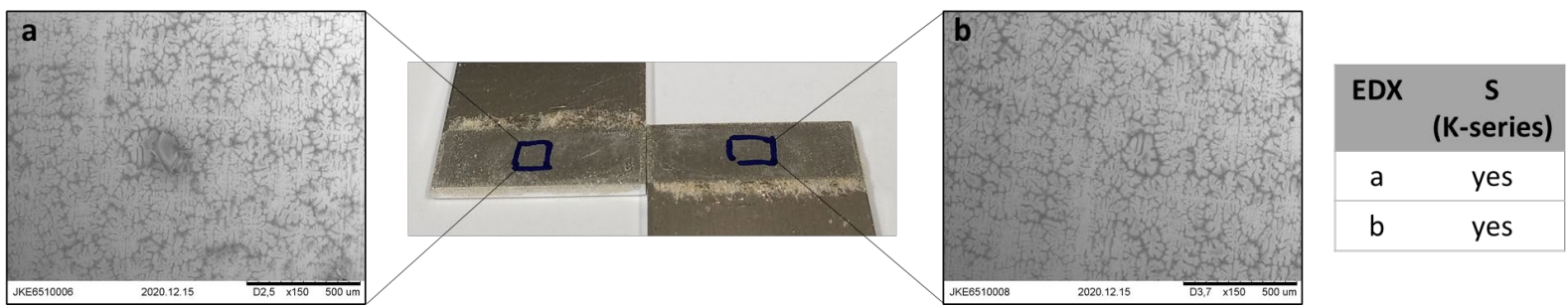
- $\mathrm{p}(\mathrm{BQA}-\mathrm{DMP})$ :
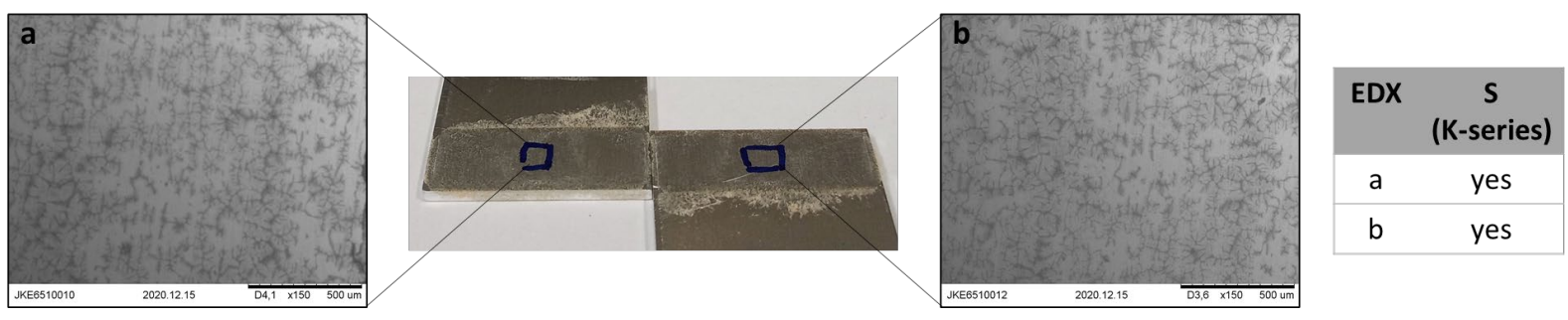

- $\mathrm{p}(\mathrm{BQA}-\mathrm{GDMA})$.
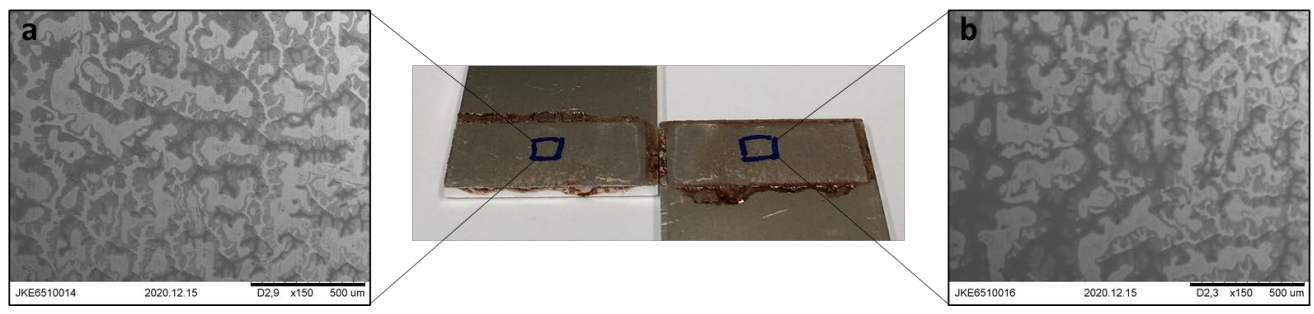

\begin{tabular}{|cc|}
\hline EDX & $\begin{array}{c}\text { S } \\
\text { (K-series) }\end{array}$ \\
\hline a & yes \\
\hline b & yes \\
\hline
\end{tabular}

- $p(B Q A-G D M P)$ :
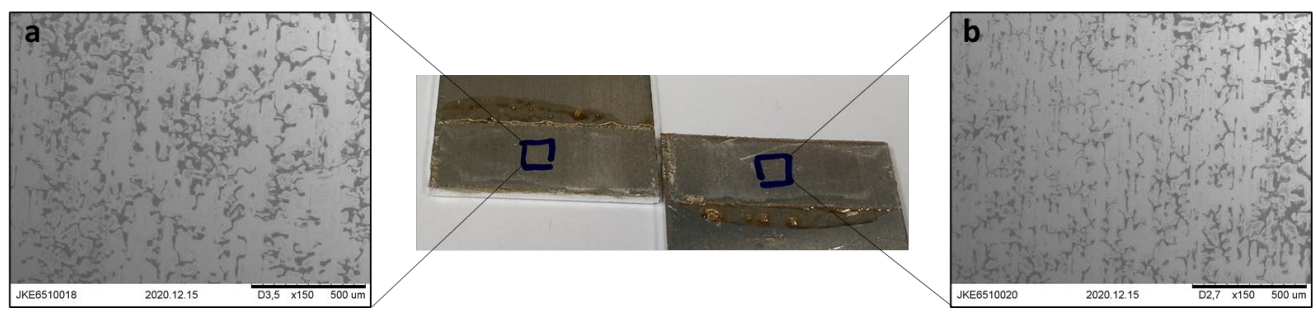

\begin{tabular}{|cc|}
\hline EDX & $\begin{array}{c}\text { S } \\
\text { (K-series) }\end{array}$ \\
\hline a & yes \\
\hline b & yes \\
\hline
\end{tabular}

- $\mathrm{p}(\mathrm{BQA}-\mathrm{BDT})$ :
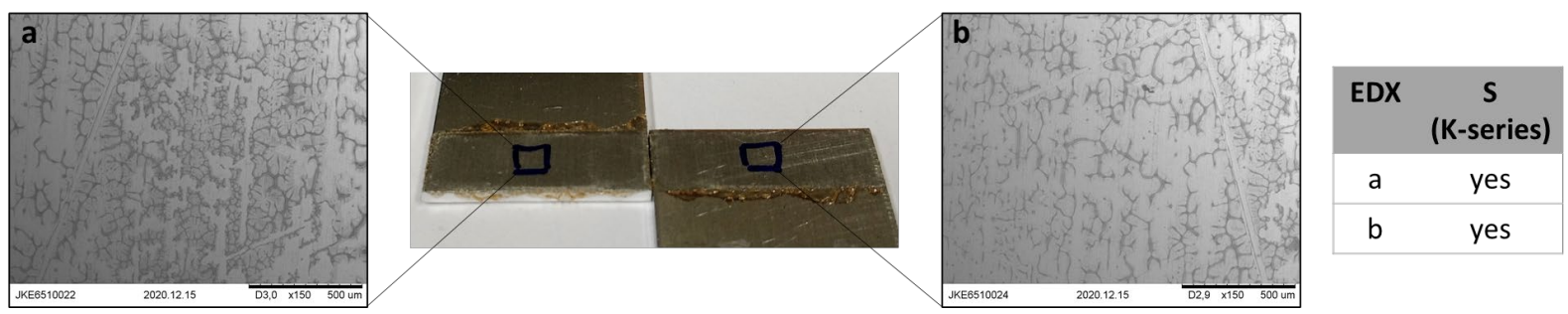

- $\mathrm{p}(\mathrm{BQA}-\mathrm{HDT})$ :
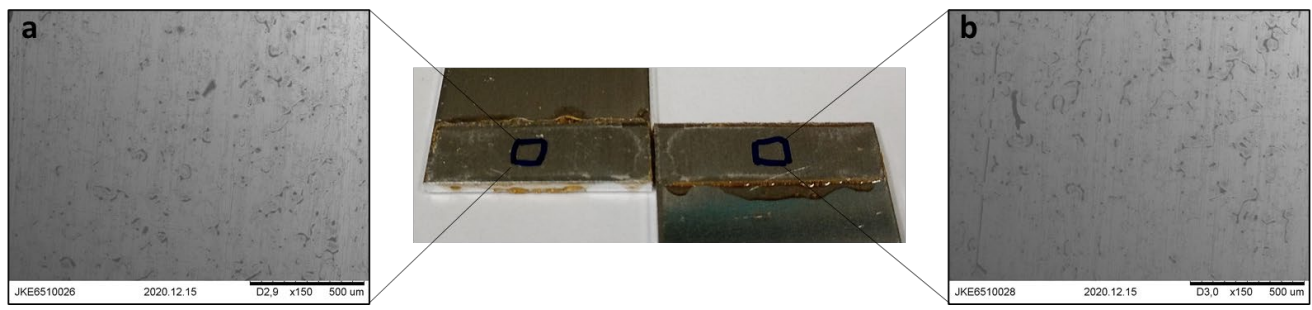

\begin{tabular}{|cc|}
\hline EDX & $\begin{array}{c}\text { S } \\
\text { (K-series) }\end{array}$ \\
\hline a & yes \\
\hline b & yes \\
\hline
\end{tabular}


- $\mathrm{p}(\mathrm{BQZ}-E D E T)$ :
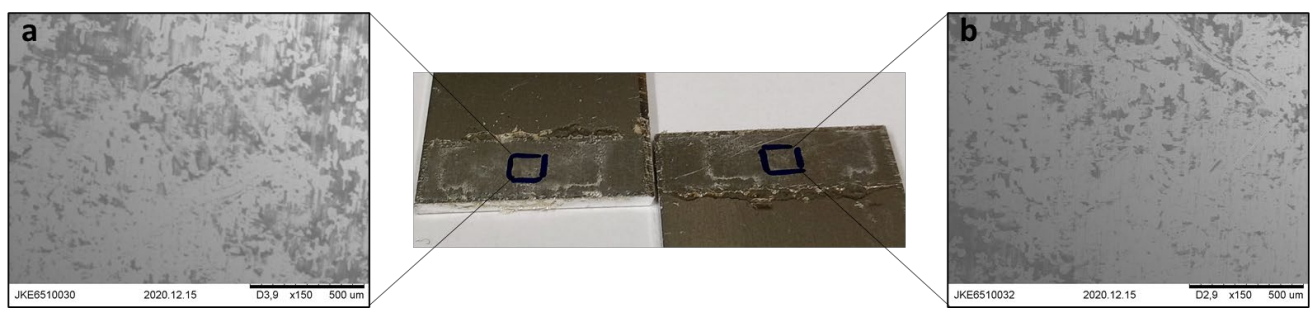

\begin{tabular}{|cc|}
\hline EDX & $\begin{array}{c}\text { S } \\
\text { (K-series) }\end{array}$ \\
\hline a & yes \\
\hline b & yes \\
\hline
\end{tabular}

- $\mathrm{p}(\mathrm{BQZ}-\mathrm{BMT})$ :
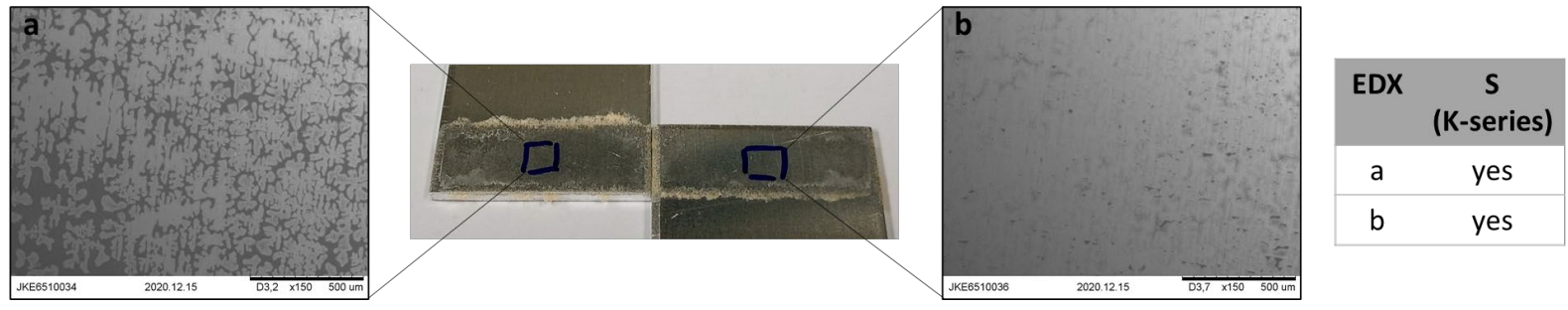

- $\mathrm{p}(\mathrm{BQZ}-\mathrm{DMP})$ :
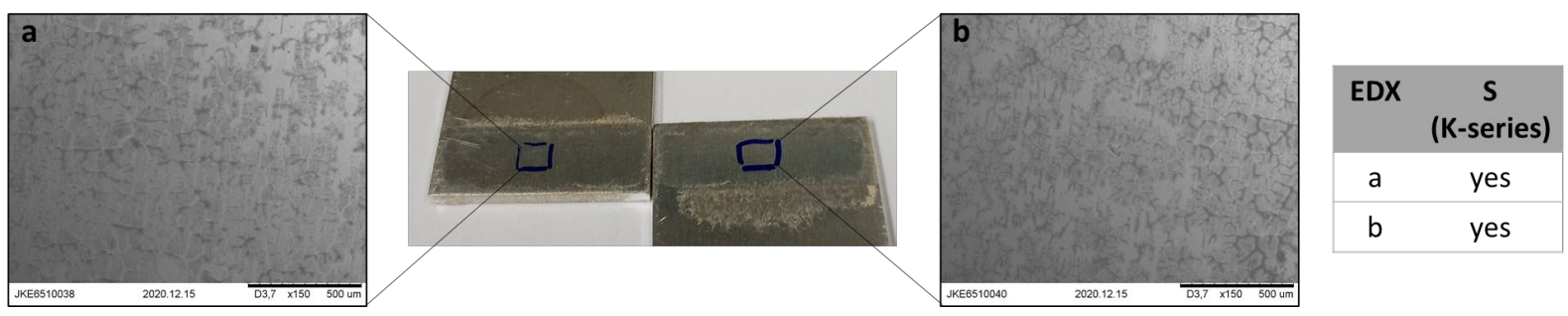

- $p(B Q Z-G D M P)$ :
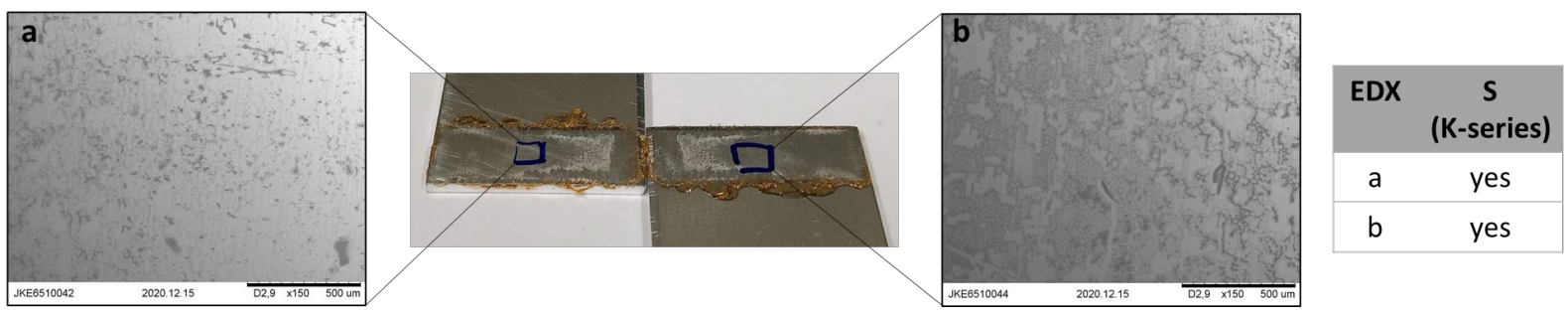

- $p(B Q Z-H D T)$ :
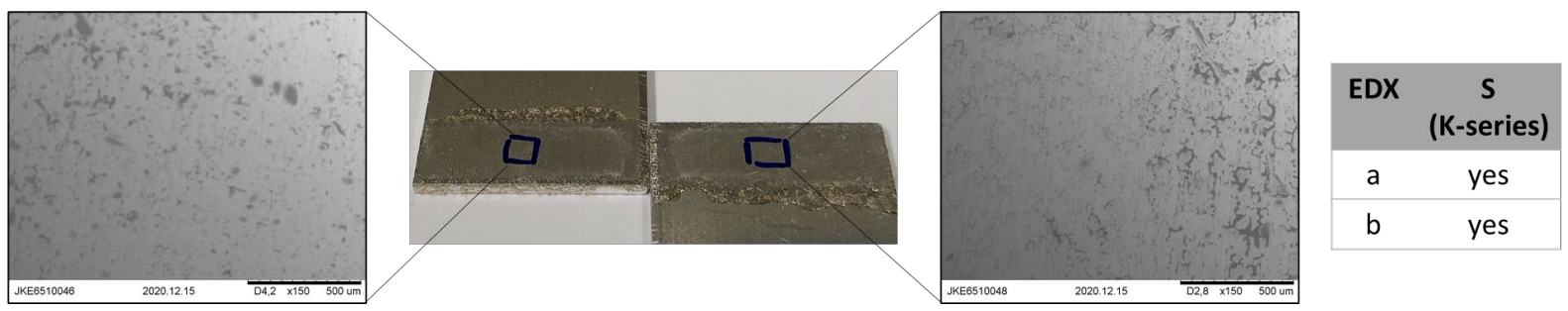
- $\mathrm{p}(\mathrm{BQB}-E D E T)$ :
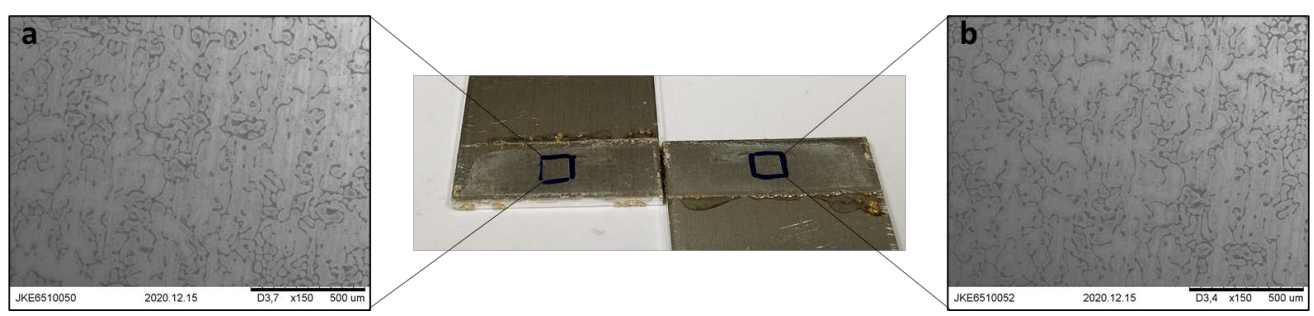

$$
\begin{array}{cc}
\hline \text { EDX } & \begin{array}{c}
\text { S } \\
\text { (K-series) }
\end{array} \\
\hline \text { a } & \text { yes } \\
\hline \text { b } & \text { yes } \\
\hline
\end{array}
$$

- $\mathrm{p}(\mathrm{BQM}-E D E T)$ :
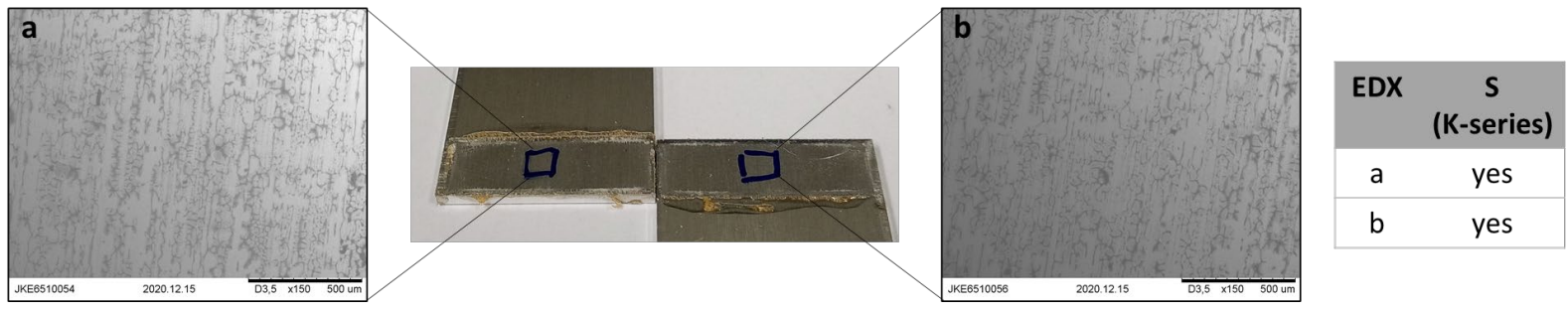

- $\mathrm{p}(\mathrm{BQM}-\mathrm{BMT})$.
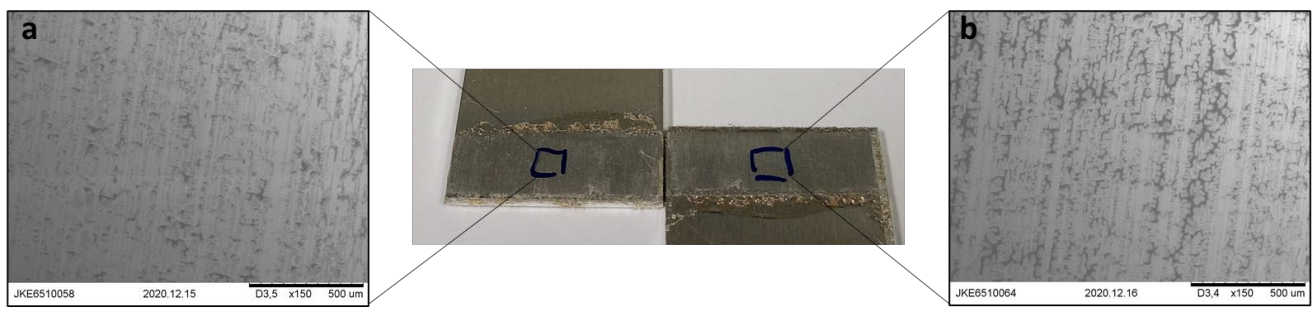

\begin{tabular}{|cc|}
\hline EDX & $\begin{array}{c}\text { S } \\
\text { (K-series) }\end{array}$ \\
\hline a & yes \\
\hline b & yes \\
\hline
\end{tabular}

- $\mathrm{p}(\mathrm{BQM}-\mathrm{DMP})$ :
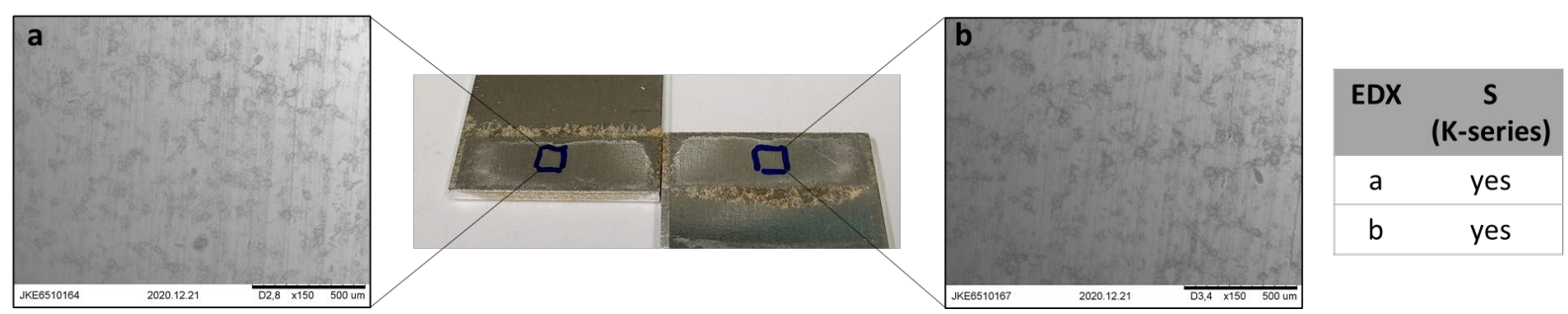

- $\mathrm{p}(\mathrm{BQM}-G D M P)$.
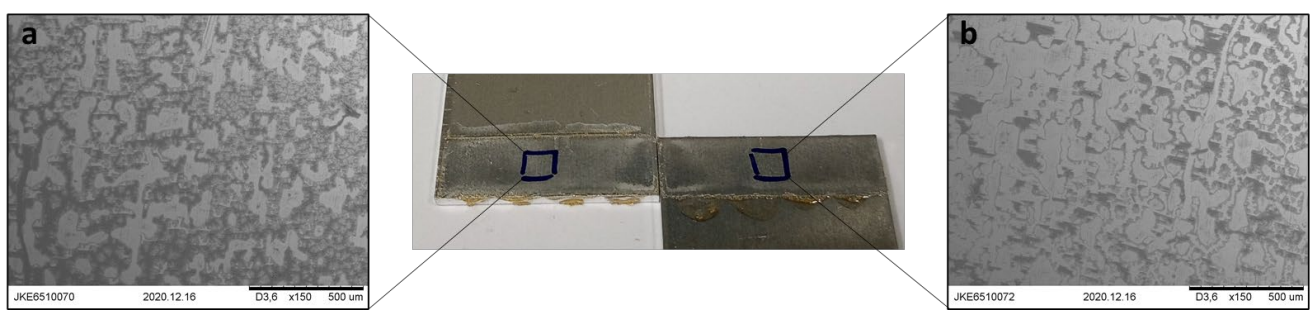

\begin{tabular}{|cc|}
\hline EDX & $\begin{array}{c}\text { S } \\
\text { (K-series) }\end{array}$ \\
\hline a & yes \\
\hline b & yes \\
\hline
\end{tabular}


- $\mathrm{p}(\mathrm{BQM}-\mathrm{HDT})$ :
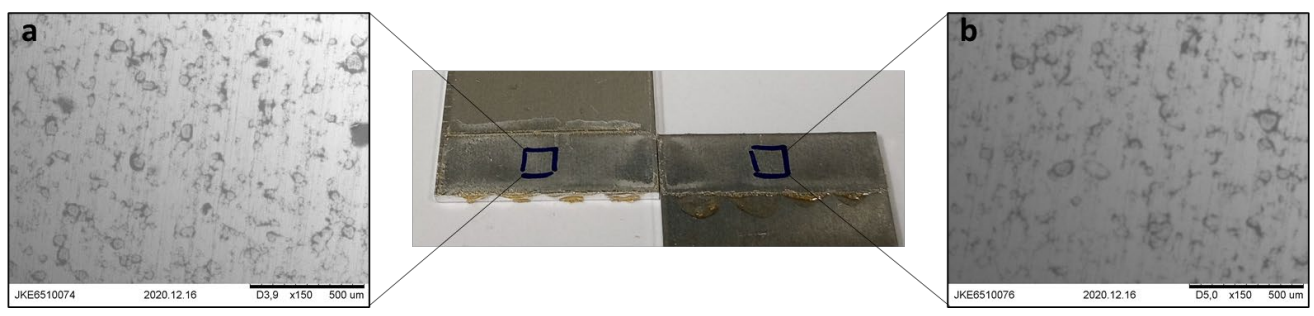

\begin{tabular}{|cc|}
\hline EDX & $\begin{array}{c}\text { S } \\
\text { (K-series) }\end{array}$ \\
\hline a & yes \\
\hline b & yes \\
\hline
\end{tabular}

The results obtained for bonding poly(propylene) are given below:

- uncoated poly(propylene):

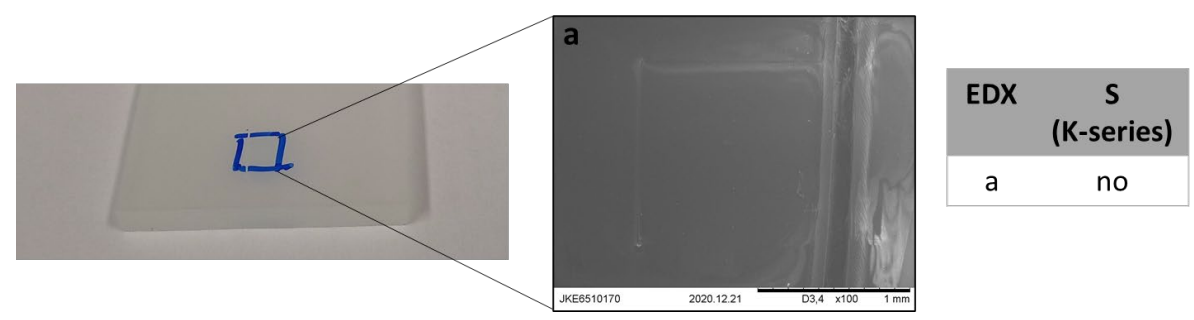

- $\mathrm{p}(\mathrm{BQA}-E D E T)$ :
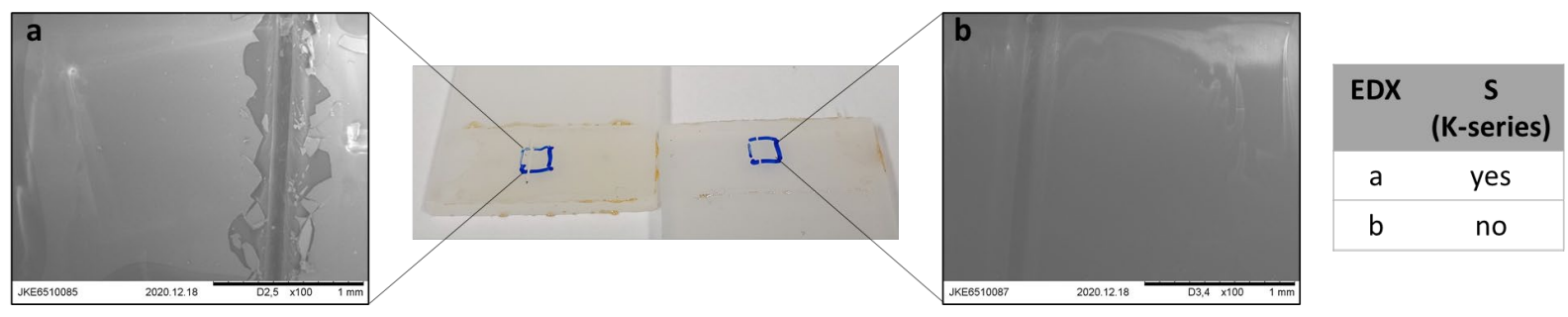

- $\mathrm{p}(\mathrm{BQA}-\mathrm{BMT})$ :
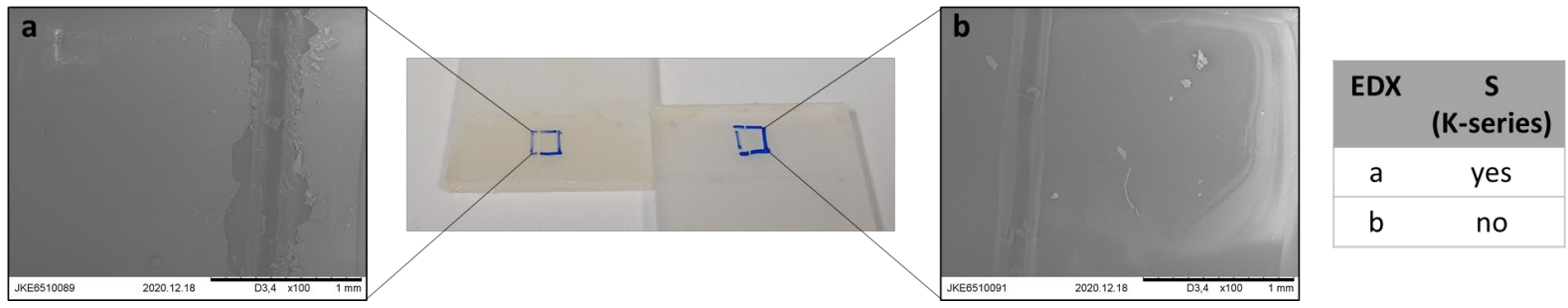

- $\mathrm{p}(\mathrm{BQA}-\mathrm{DMP})$ :
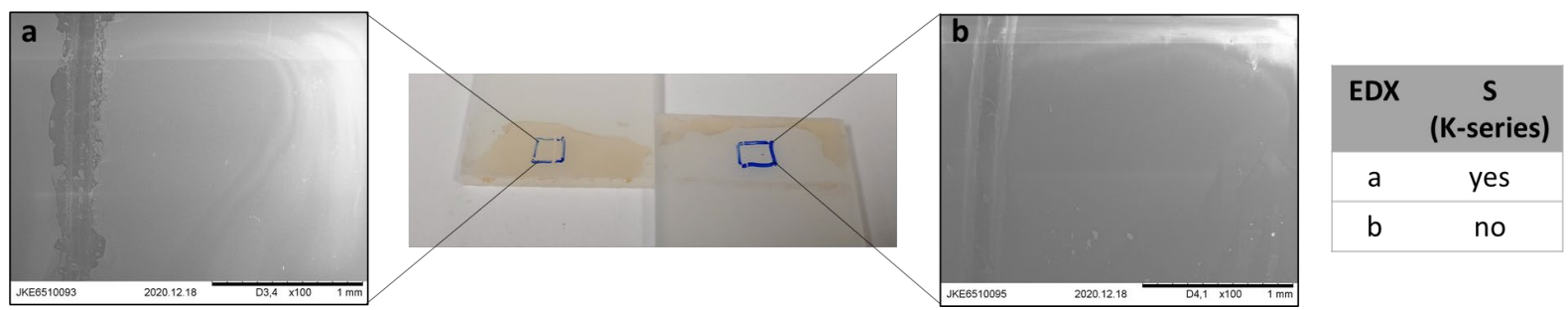
- $\mathrm{p}(\mathrm{BQA}-\mathrm{GDMA})$ :
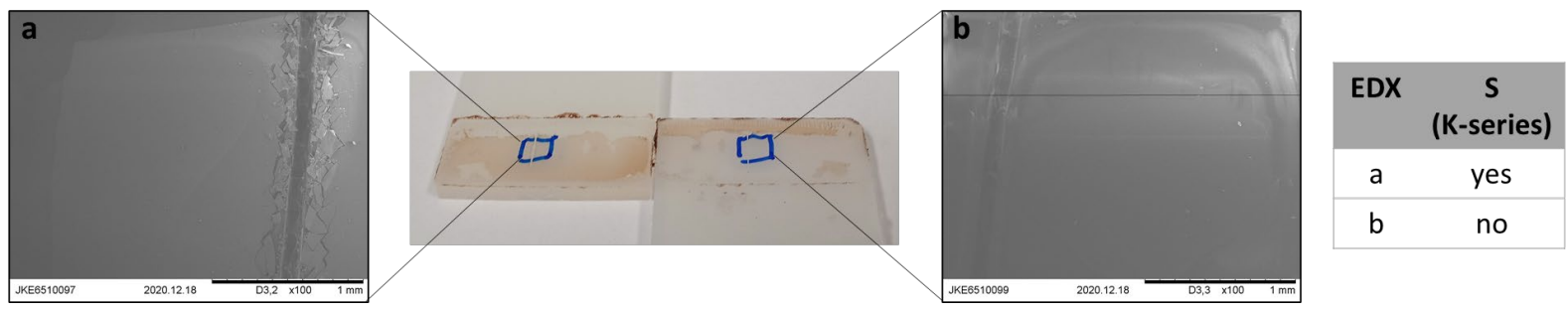

- $\mathrm{p}(\mathrm{BQA}-\mathrm{GDMP})$ :
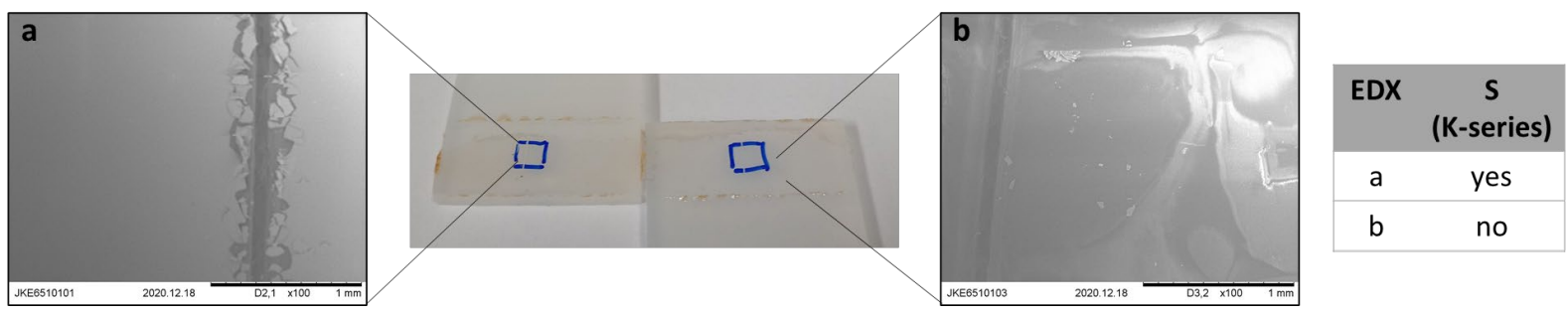

- $\quad \mathrm{p}(\mathrm{BQA}-\mathrm{BDT})$ :
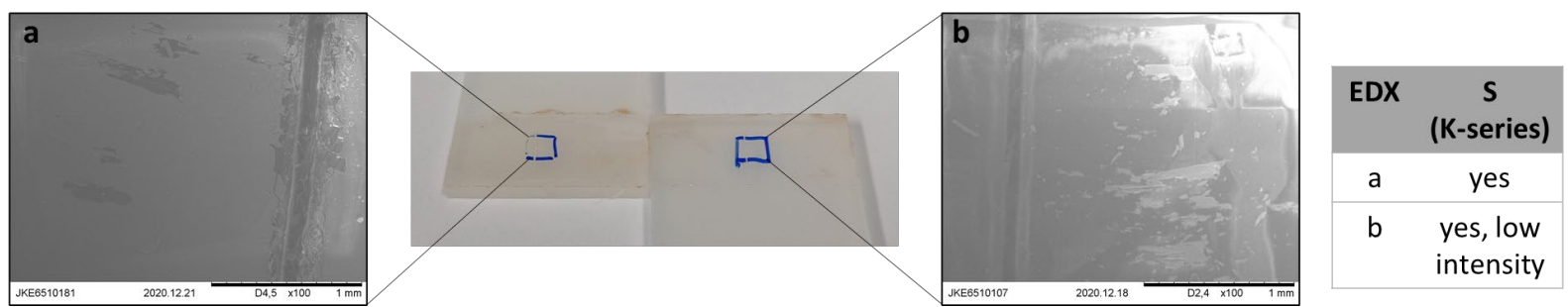

- $\mathrm{p}(\mathrm{BQA}-H D T)$ :
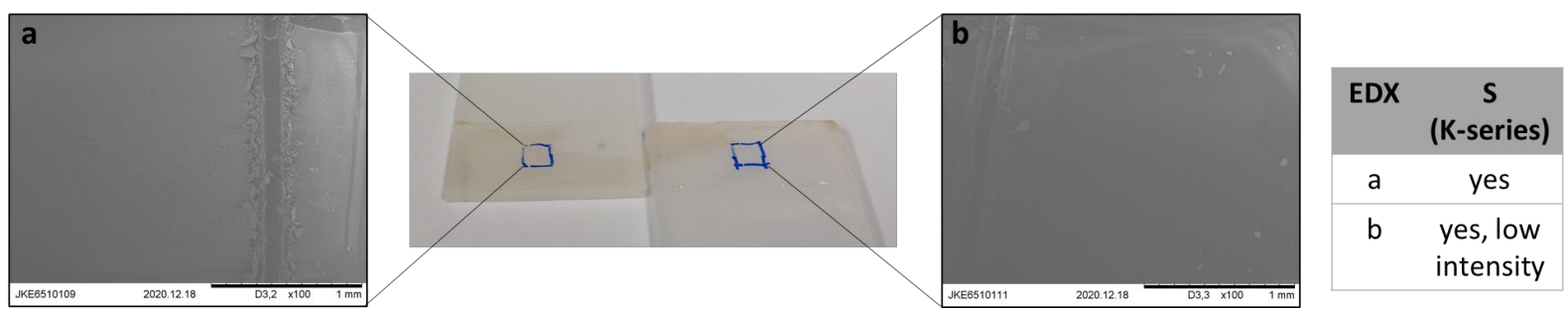

- $\quad \mathrm{p}(\mathrm{BQZ}-E D E T)$ :
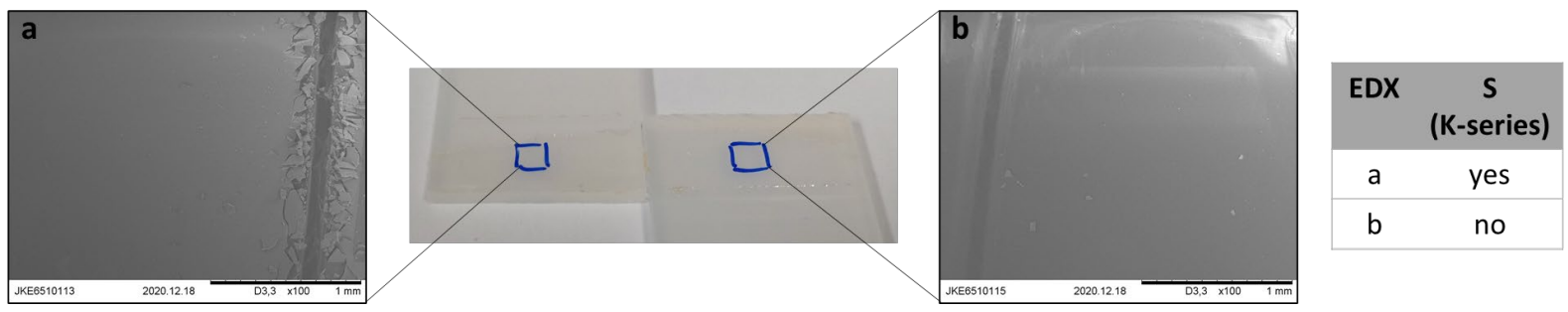
- $\mathrm{p}(\mathrm{BQZ}-\mathrm{BMT})$ :
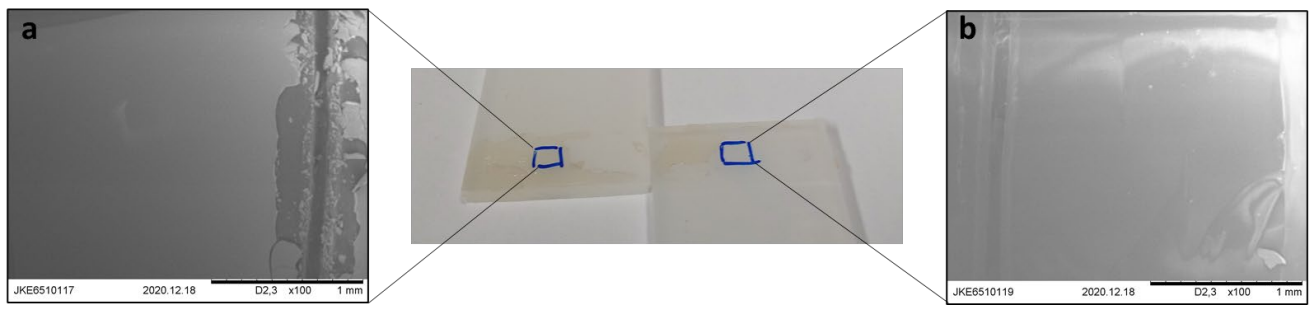

$$
\begin{array}{cc}
\text { EDX } & \text { S } \\
& \text { (K-series) } \\
\hline \text { a } & \text { yes } \\
\hline \text { b } & \text { no }
\end{array}
$$

- $\mathrm{p}(\mathrm{BQZ}-\mathrm{DMP})$ :
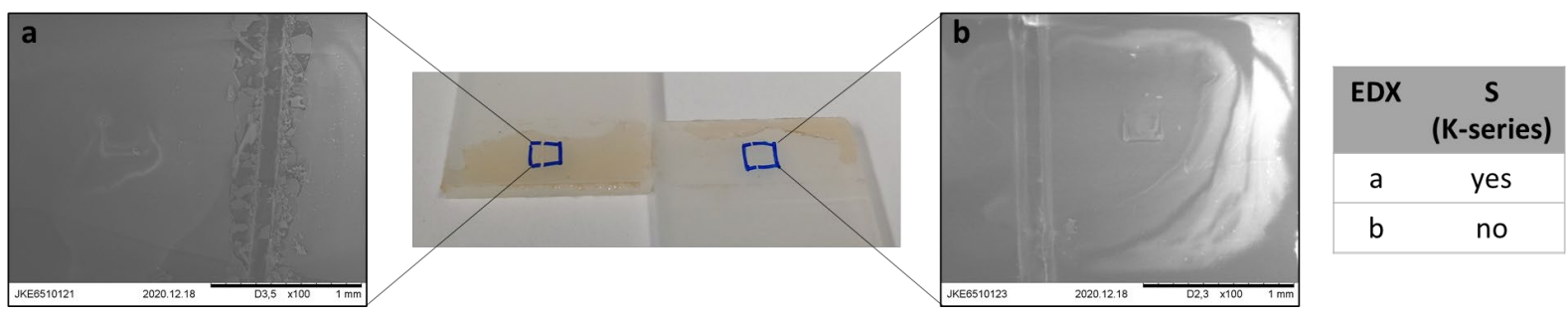

- $\mathrm{p}(\mathrm{BQZ}-\mathrm{GDMP})$ :
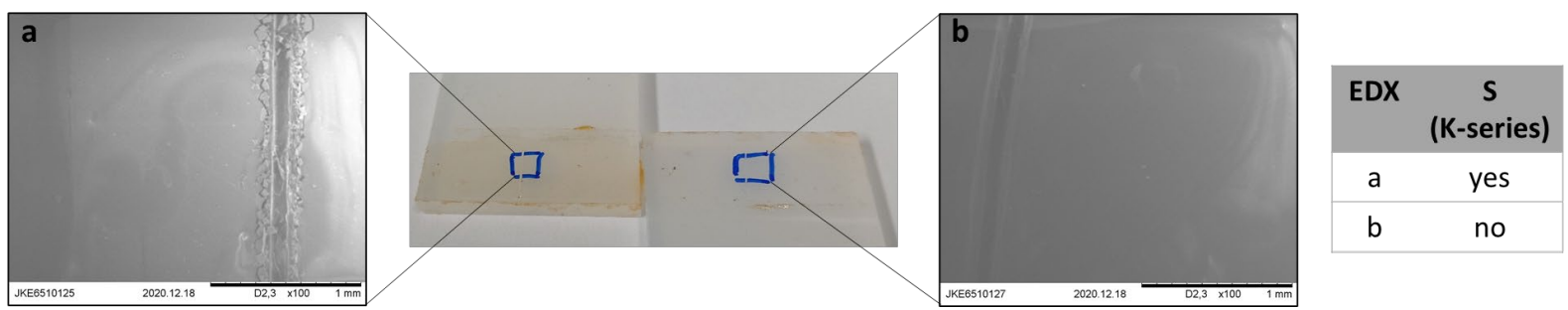

- $p(B Q Z-H D T)$ :
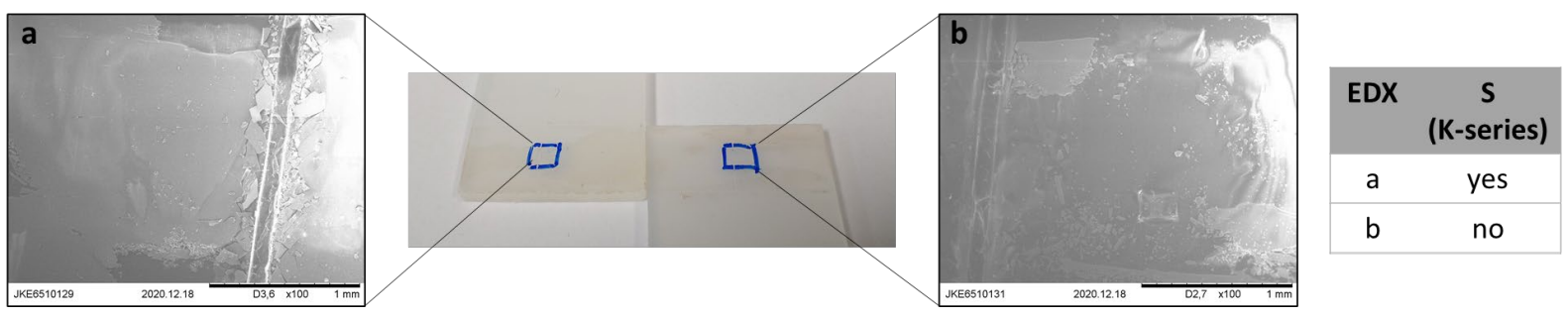

- $\mathrm{p}(\mathrm{BQB}-E D E T)$.
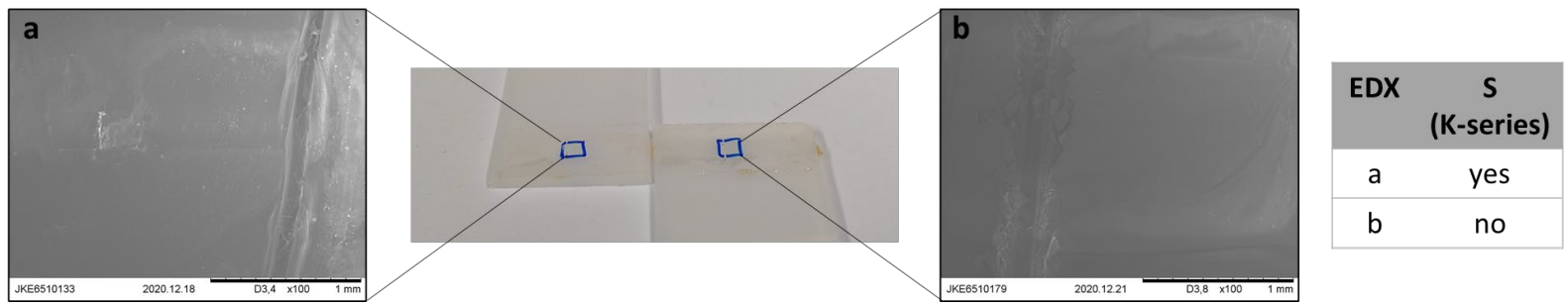
- $\mathrm{p}(\mathrm{BQM}-E D E T)$ :
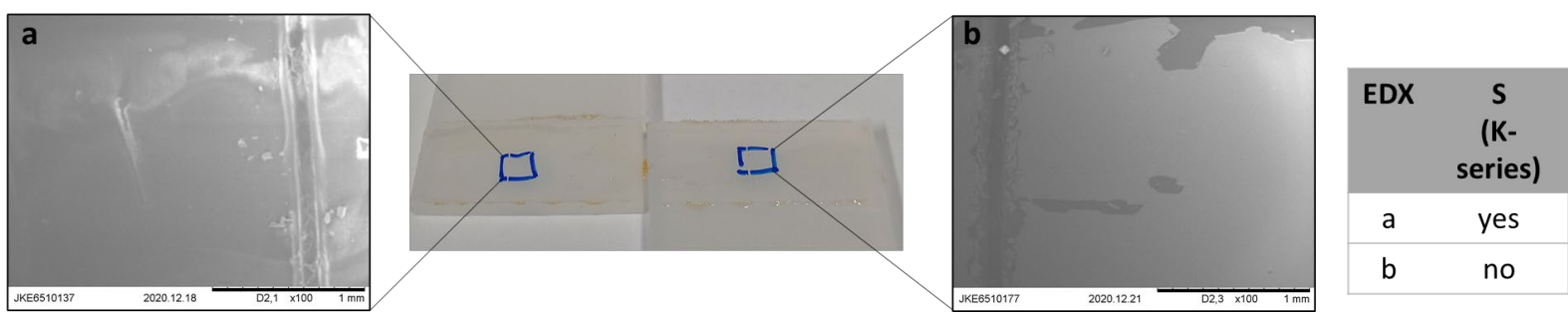

- $\mathrm{p}(\mathrm{BQM}-\mathrm{BMT})$ :
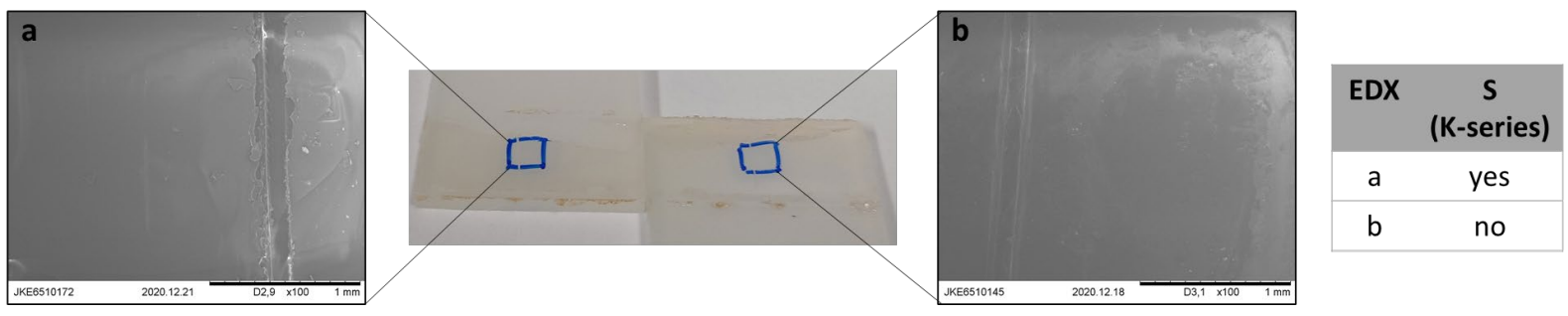

- $\mathrm{p}(\mathrm{BQM}-\mathrm{DMP})$ :
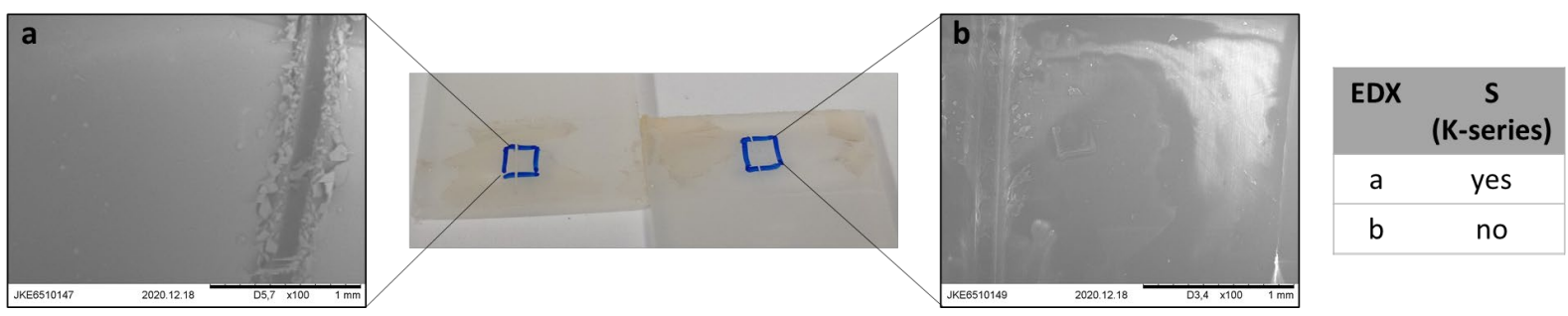

- $\mathrm{p}(\mathrm{BQM}-G D M P)$ :
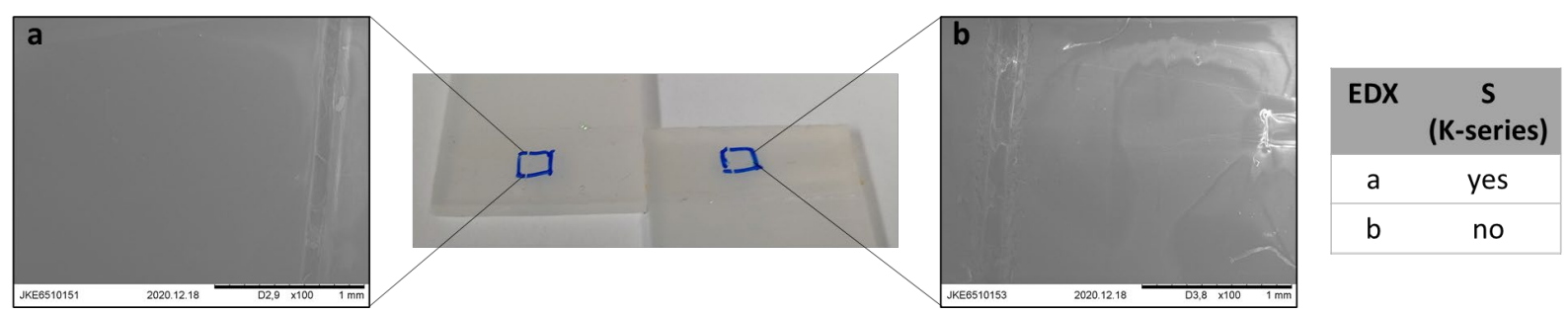

- $\mathrm{p}(\mathrm{BQM}-H D T)$ :
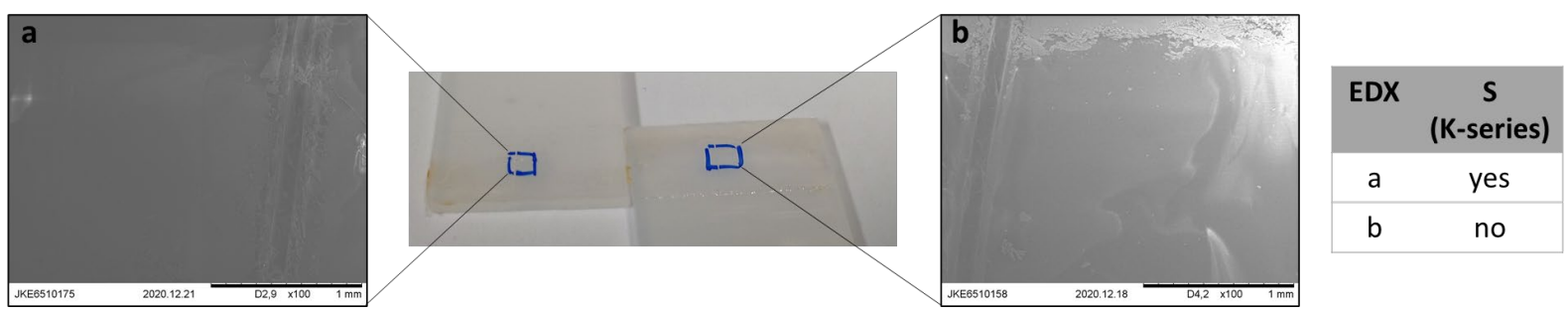


\subsection{Lap shear test of TCC-polymers under wet conditions}

Lap shear tests were carried out according to the procedure in section 3.3 and 3.4.

Table S 7. Adhesive strength values for lap shear tests of TCC-polymer adhesive tests in $599 \mathrm{mM} \mathrm{NaCl-solution.}$

\begin{tabular}{|c|c|}
\hline polymer & adhesive strength after curing in $\mathbf{5 9 9} \mathbf{~ m M ~ N a C l - s o l u t i o n}$ \\
\hline p(DCB-EDET) & {$[\mathbf{M P a}]$} \\
\hline p(DCZ-EDET) & $0.99 \pm 0.29$ \\
\hline p(DCM-EDET) & $0.55 \pm 0.22$ \\
\hline p(DCM-BMT) & $1.25 \pm 0.57$ \\
\hline p(DCM-GDMP) & $1.02 \pm 0.58$ \\
\hline p(DCM-HDT) & $1.02 \pm 0.42$ \\
\hline
\end{tabular}
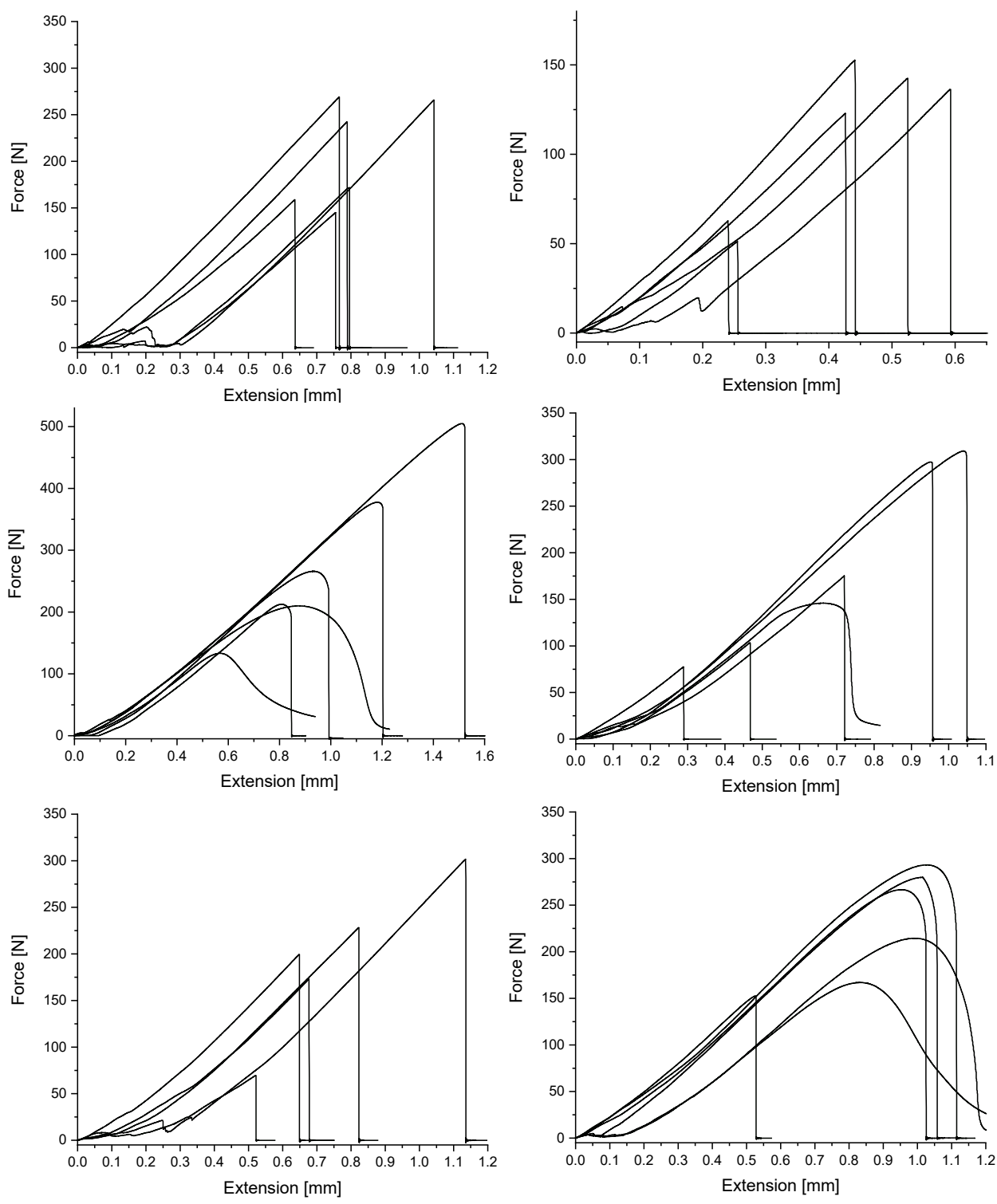

Figure S 64. Force-extension curves of lap shear tests of TCC-polymers after underwater application and curing on aluminum. $\mathrm{p}$ (BQB-EDET) (top left), $\mathrm{p}$ (BQZ-EDET) (top right), $\mathrm{p}$ (BQM-EDET) (mid left), $\mathrm{p}$ (BQM-BMT) (mid right) as well as $\mathrm{p}(\mathrm{BQM}-\mathrm{GDMP})$ (bottom left) and $\mathrm{p}(\mathrm{BQM}-\mathrm{HDT})$ (bottom right). 


\section{NMR spectra of the TCC-polymers}

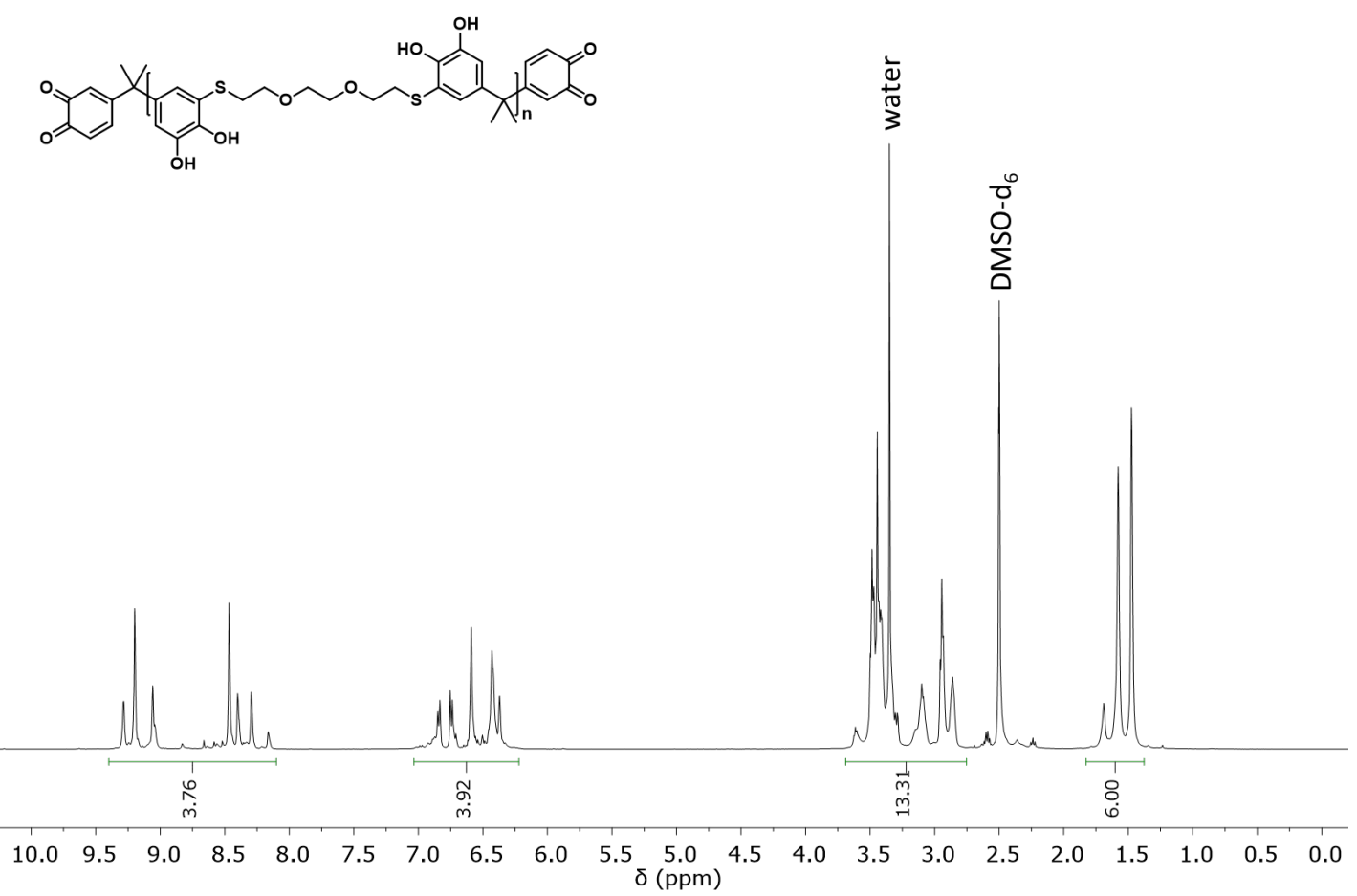

Figure S 65. ${ }^{1} \mathrm{H}-\mathrm{NMR}$-spectra of $\mathrm{p}(\mathrm{DCA}-\mathrm{EDET})$ in DMSO-d 6 .
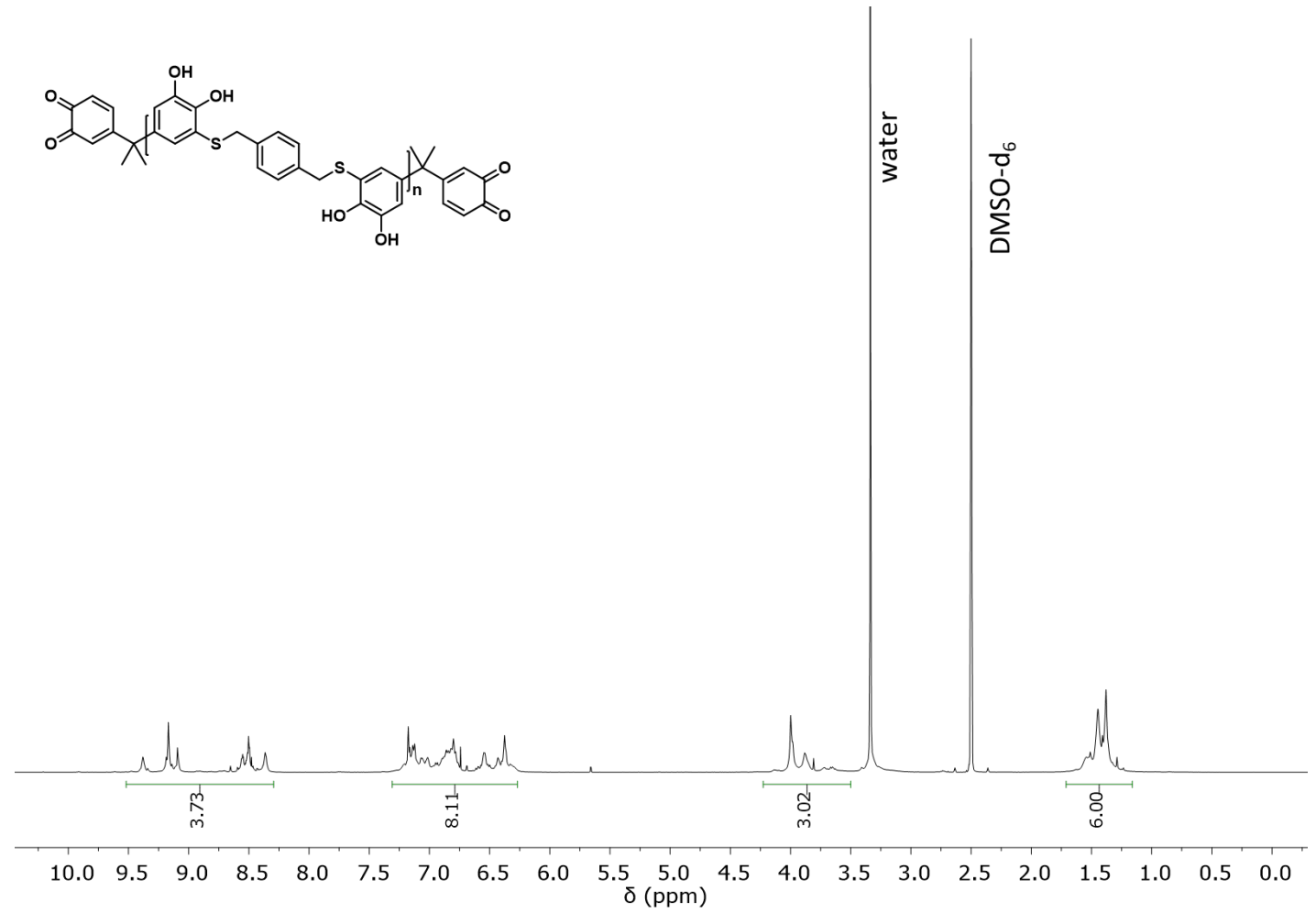

Figure S 66. ${ }^{1} \mathrm{H}-\mathrm{NMR}$-spectra of $\mathrm{p}(\mathrm{DCA}-\mathrm{BMT})$ in DMSO-d . $_{6}$ 

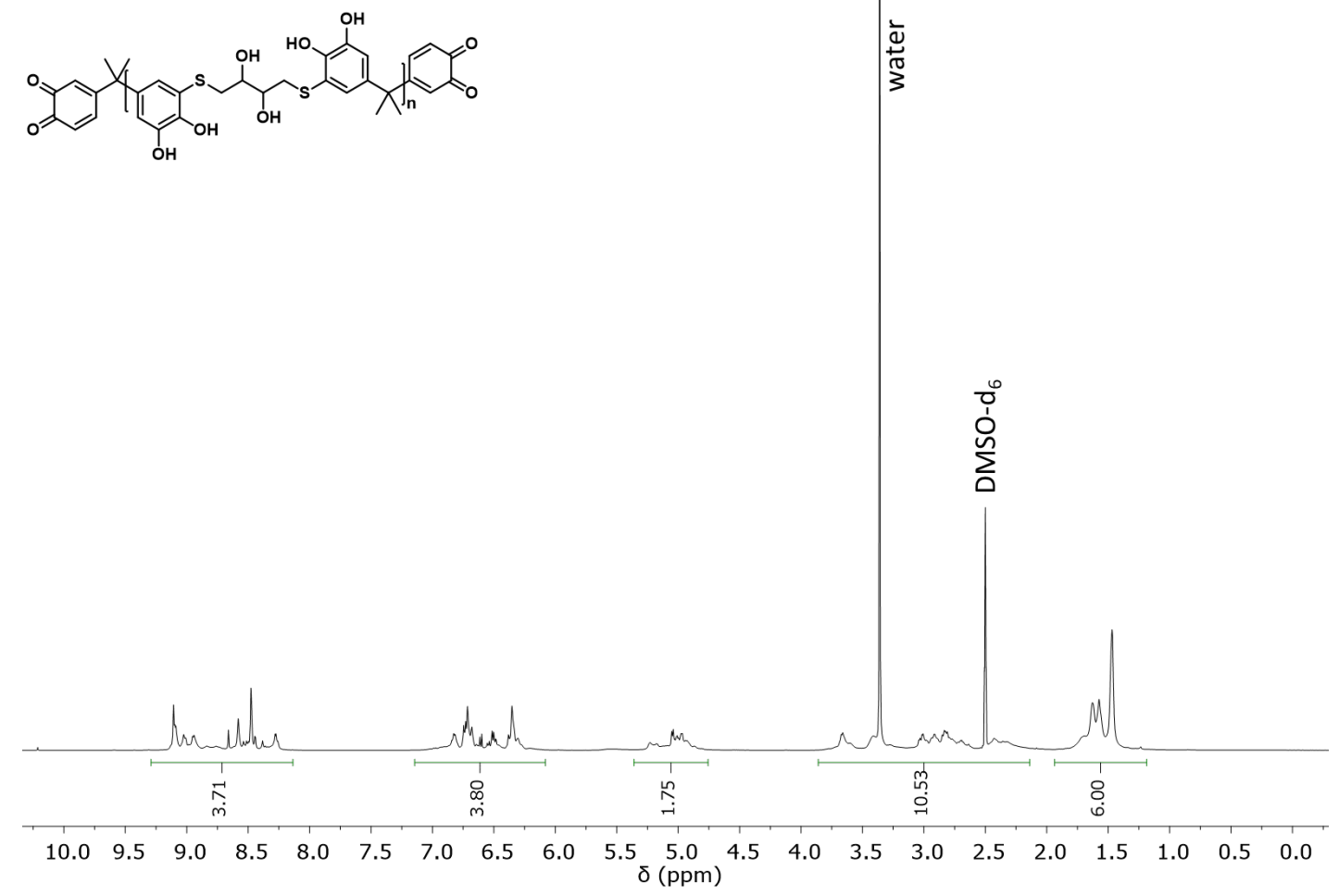

Figure S 67. ${ }^{1} \mathrm{H}-\mathrm{NMR}$-spectra of $\mathrm{p}(\mathrm{DCA}-\mathrm{DTT})$ in DMSO-d . $_{\text {. }}$

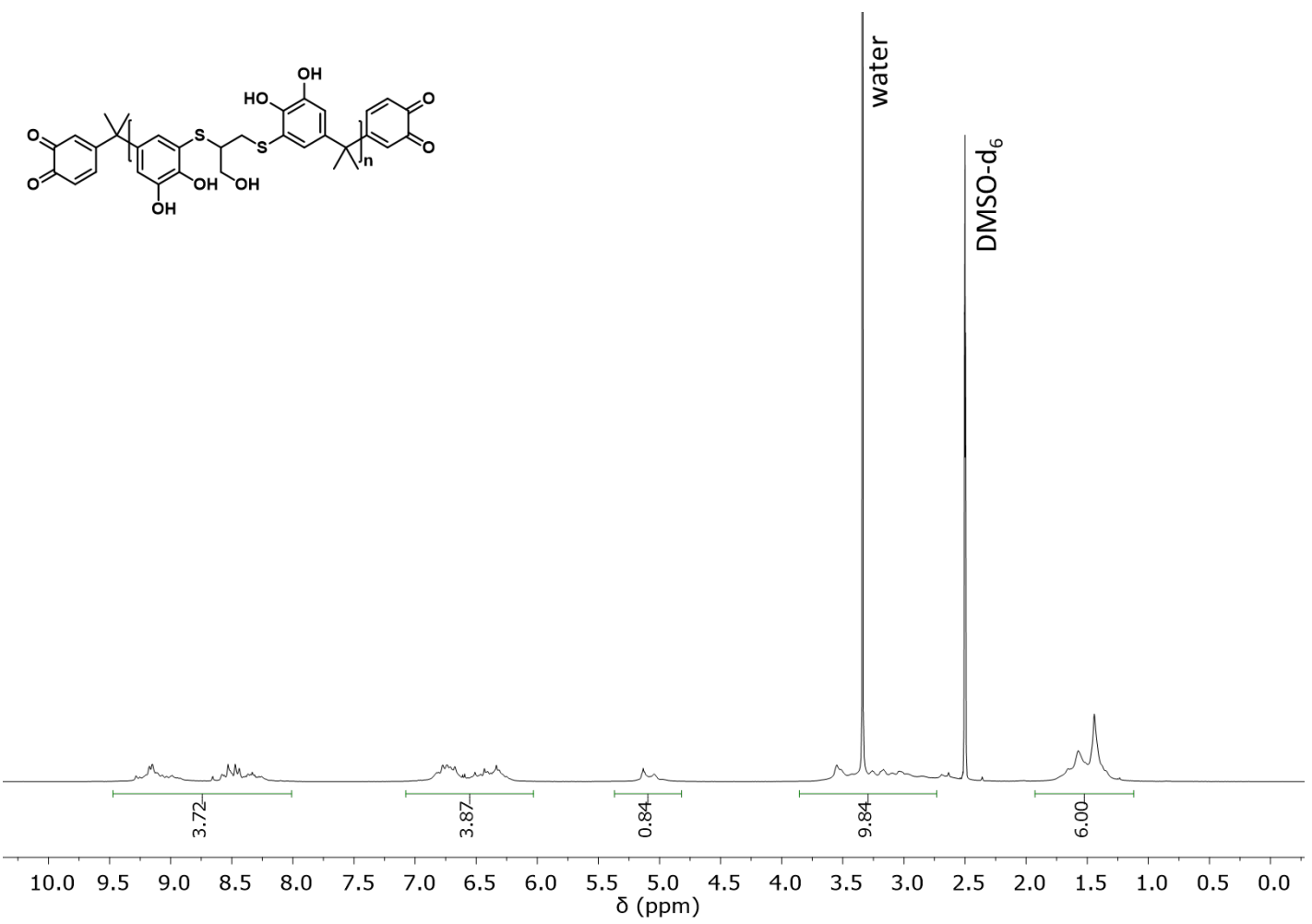

Figure S 68. ${ }^{1} \mathrm{H}-\mathrm{NMR}$-spectra of $\mathrm{p}(\mathrm{DCA}-\mathrm{DMP})$ in DMSO-d ${ }_{6}$. 


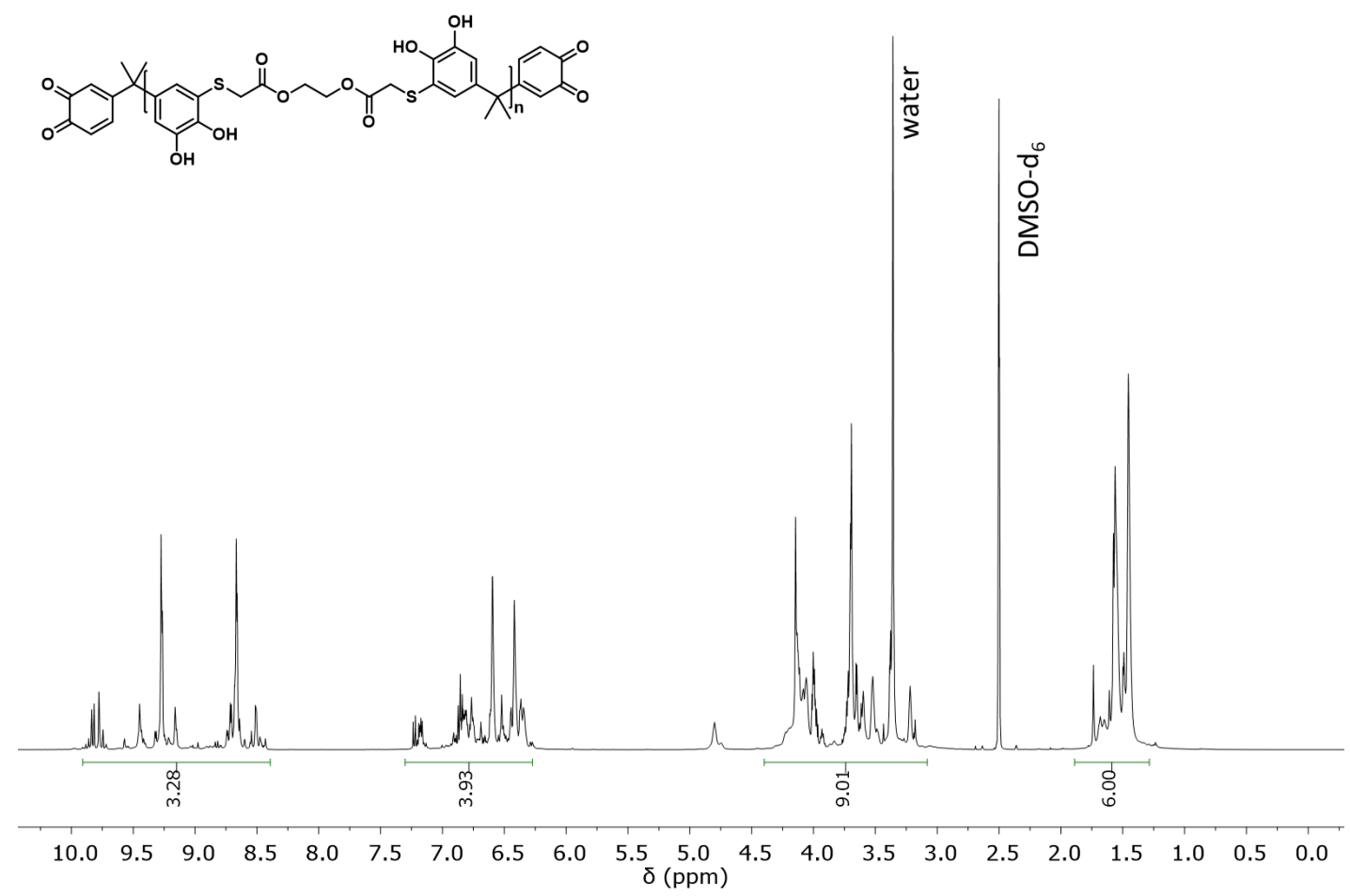

Figure S 69. ${ }^{1} \mathrm{H}-\mathrm{NMR}$-spectra of $\mathrm{p}(\mathrm{DCA}-\mathrm{GDMA})$ in DMSO-d ${ }_{6}$.
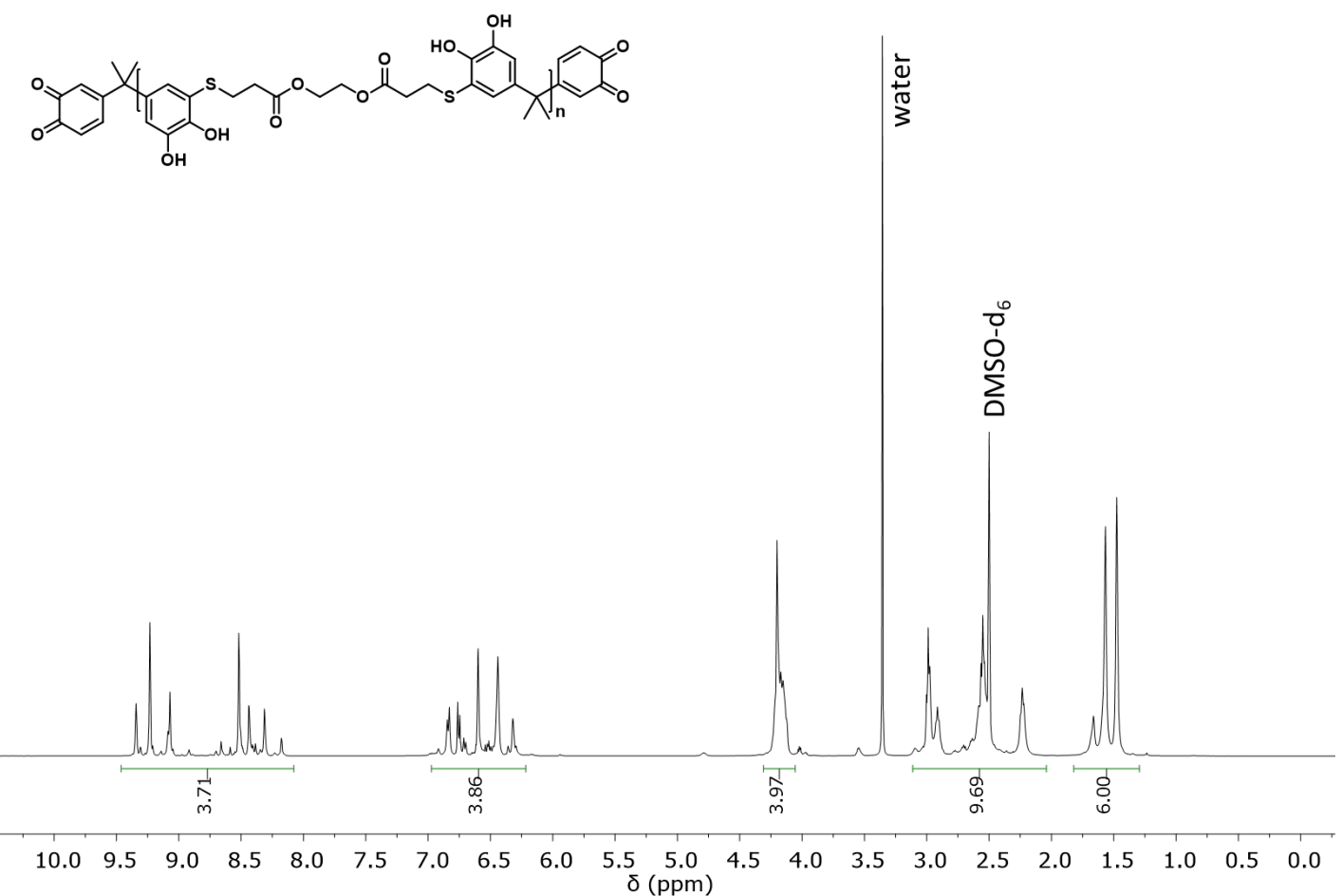

Figure $\mathrm{S} 70 .{ }^{1} \mathrm{H}-\mathrm{NMR}$-spectra of $\mathrm{p}(\mathrm{DCA}-\mathrm{GDMP})$ in DMSO-d $\mathrm{d}_{6}$. 


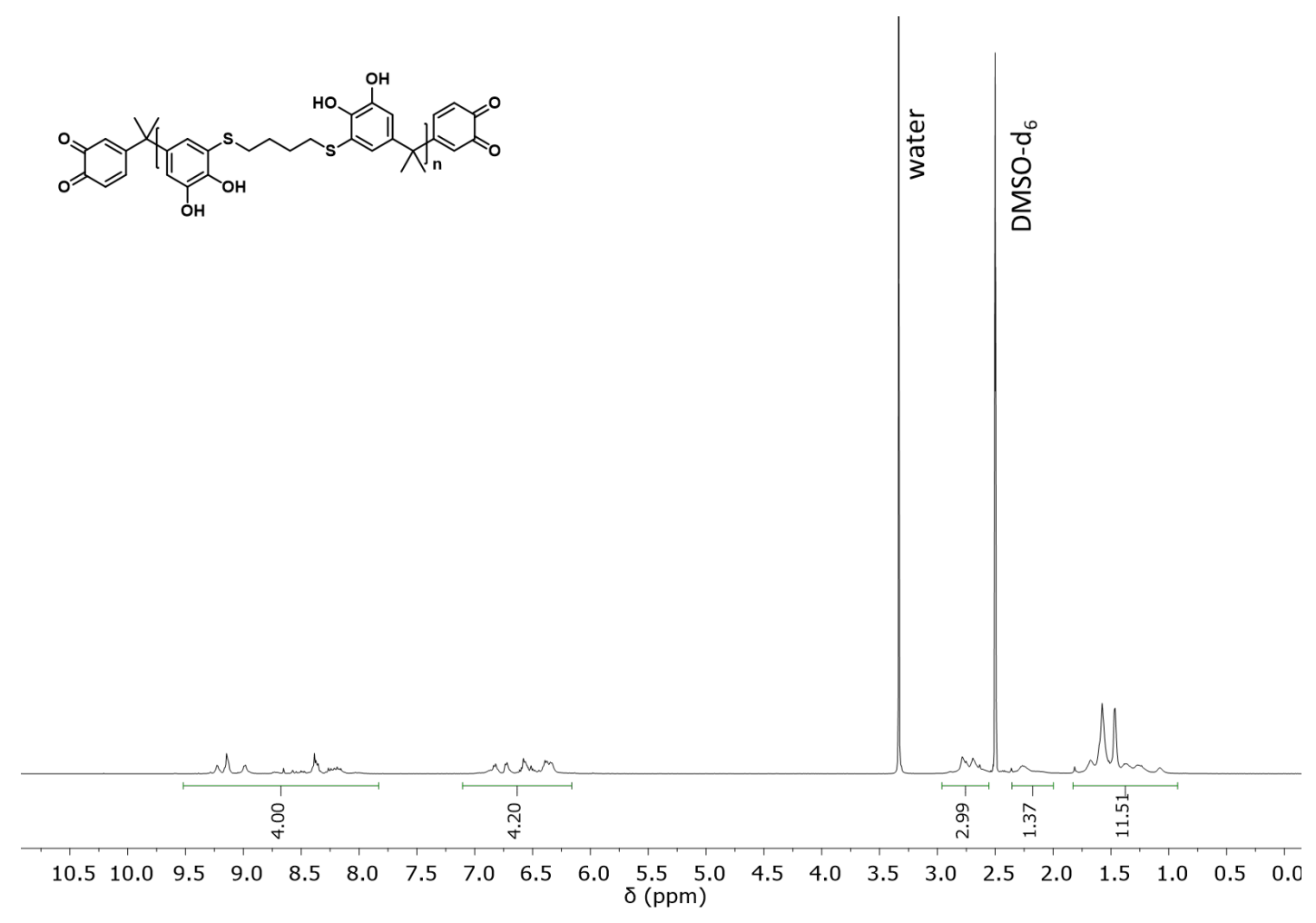

Figure S 71. ${ }^{1} \mathrm{H}-\mathrm{NMR}$-spectra of $\mathrm{p}(\mathrm{DCA}-\mathrm{BDT})$ in DMSO-d ${ }_{6}$.
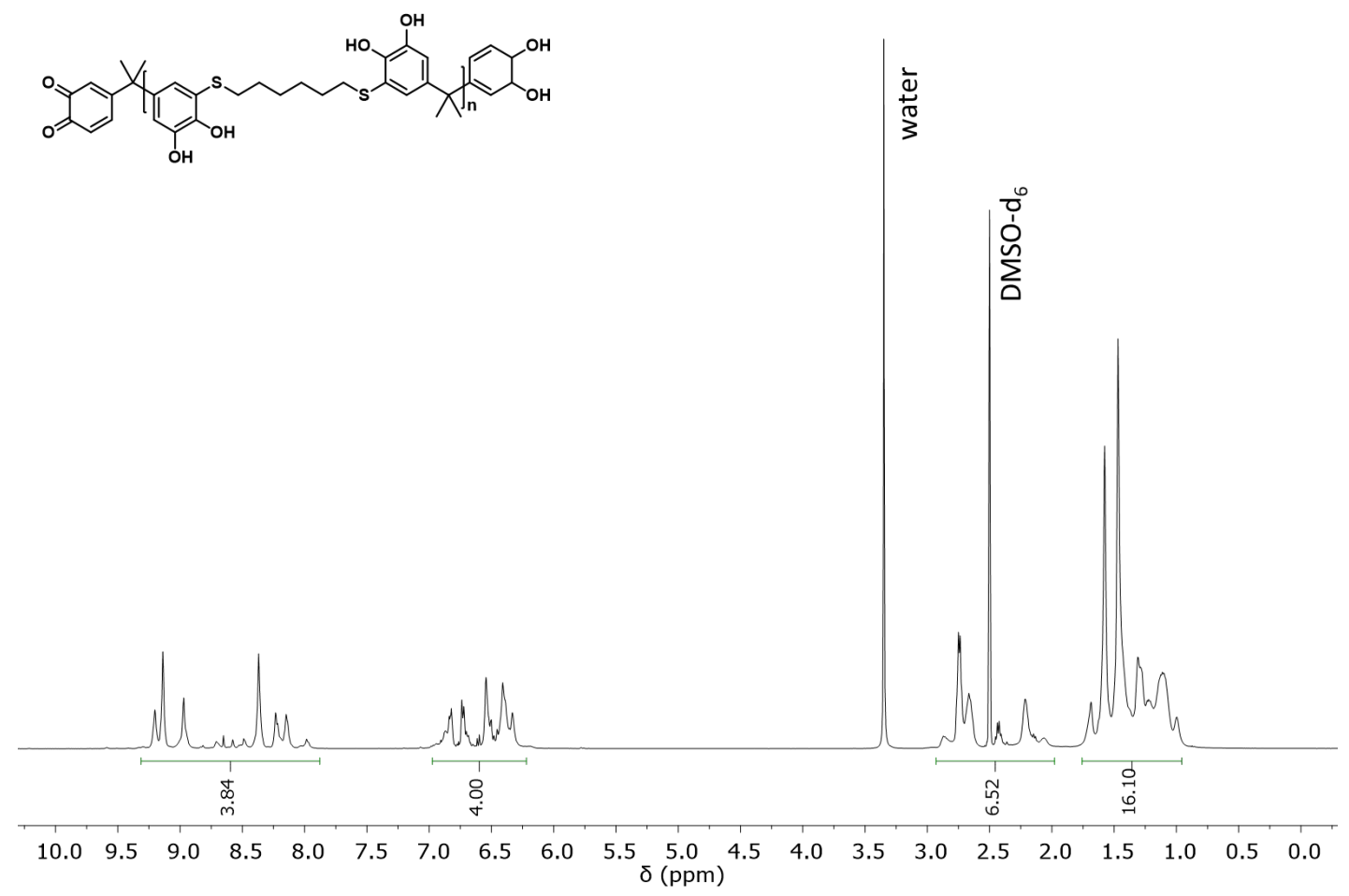

Figure S 72. ${ }^{1} \mathrm{H}-\mathrm{NMR}$-spectra of $\mathrm{p}(\mathrm{DCA}-\mathrm{HDT})$ in DMSO-d $\mathrm{d}_{6}$. 


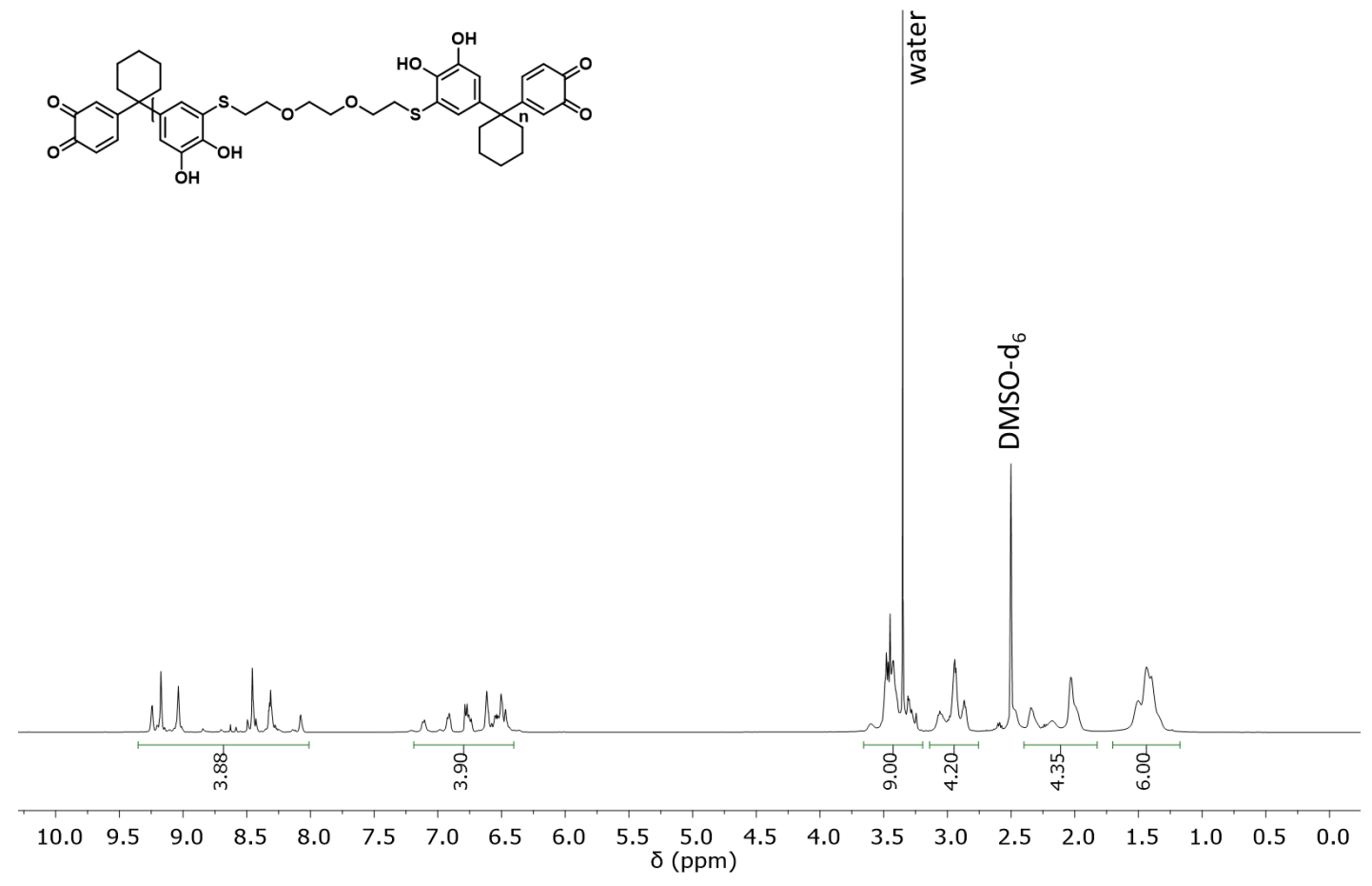

Figure S 73. ${ }^{1} \mathrm{H}-\mathrm{NMR}$-spectra of $\mathrm{p}(\mathrm{DCZ}-\mathrm{EDET})$ in DMSO-d ${ }_{6}$.

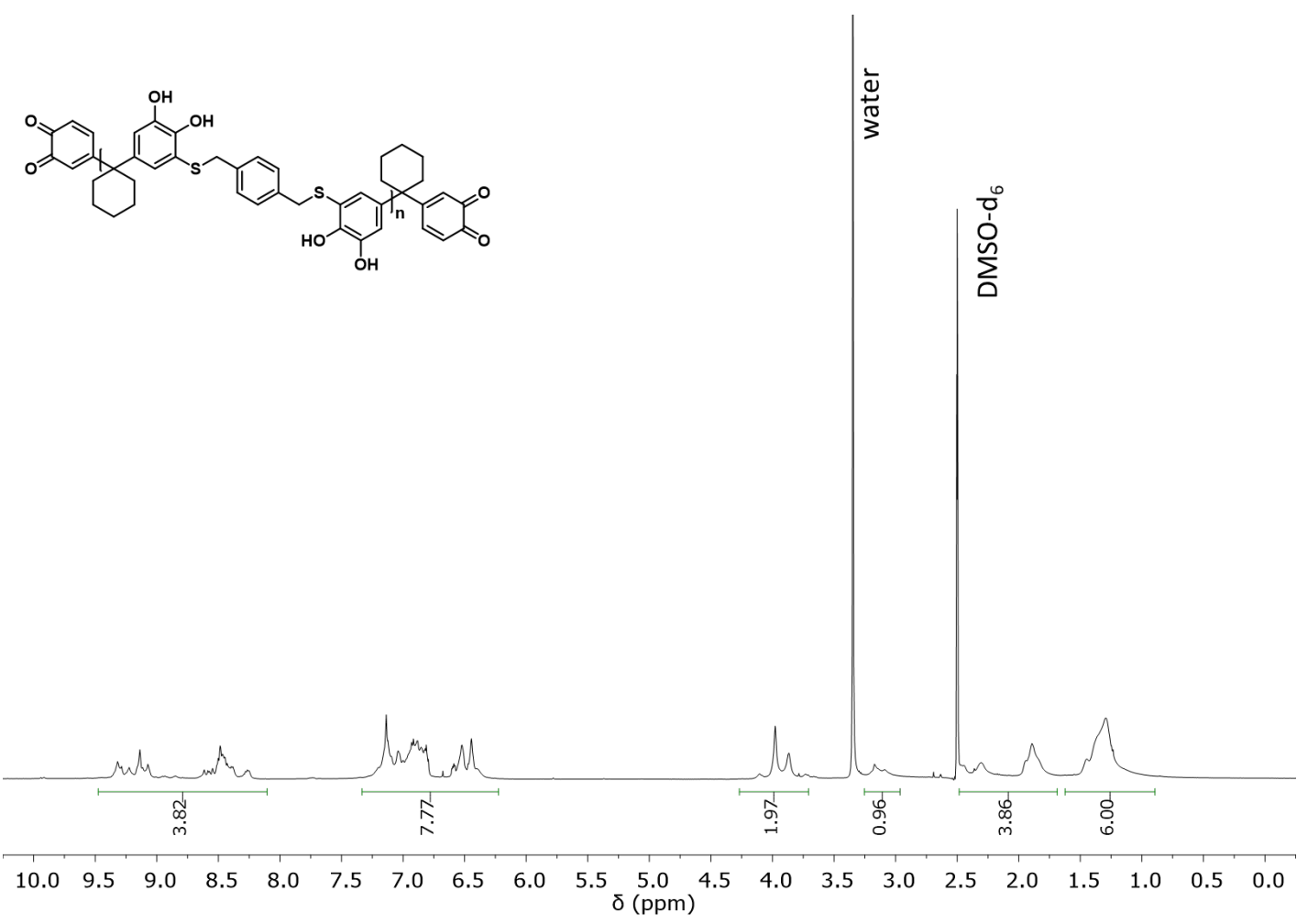

Figure S 74. ${ }^{1} \mathrm{H}-\mathrm{NMR}$-spectra of $\mathrm{p}(\mathrm{DCZ}-\mathrm{BMT})$ in DMSO-d $\mathrm{d}_{6}$. 


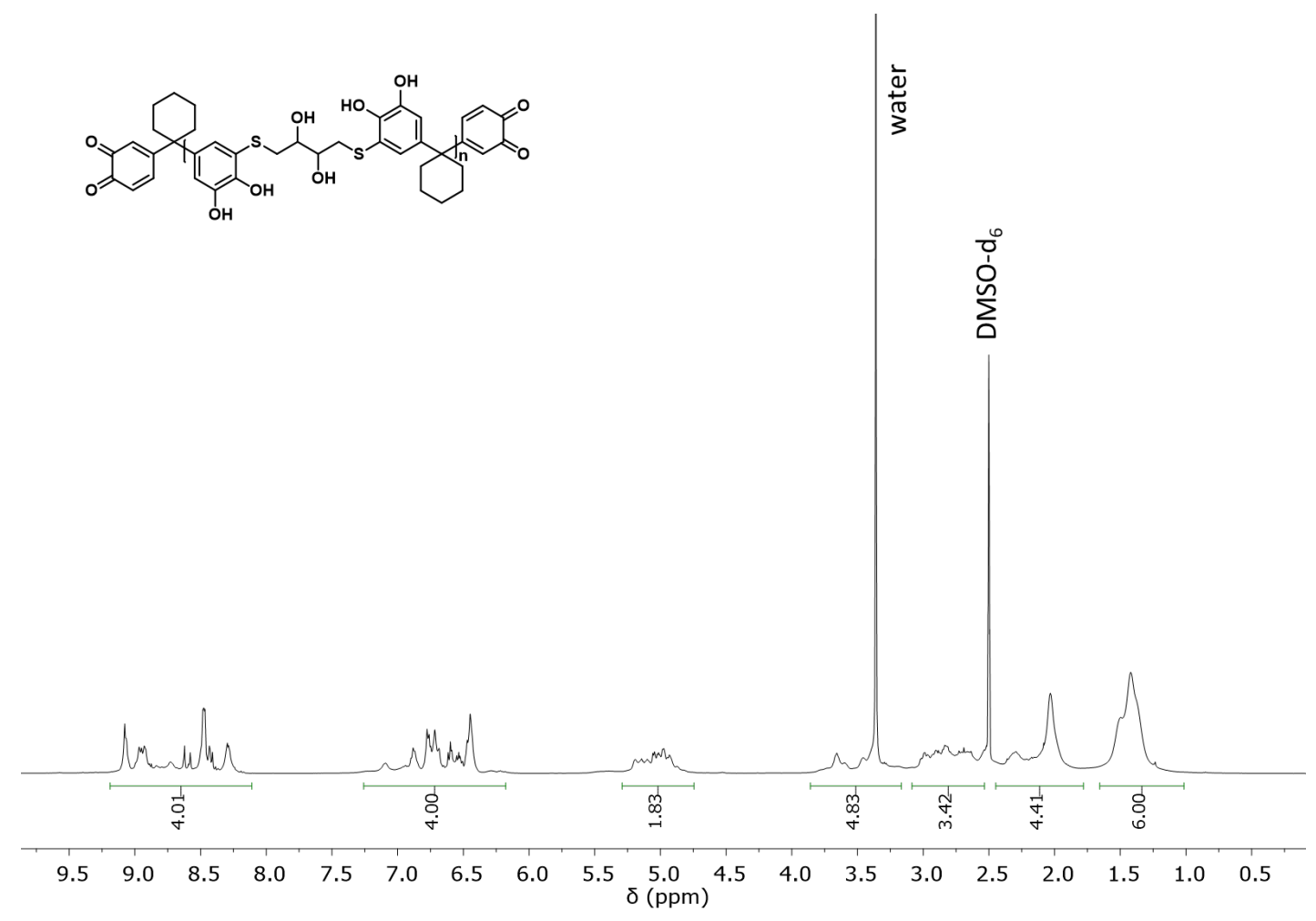

Figure S 75. ${ }^{1} \mathrm{H}-\mathrm{NMR}$-spectra of $\mathrm{p}(\mathrm{DCZ}-\mathrm{DTT})$ in $\mathrm{DMSO}-\mathrm{d}_{6}$.

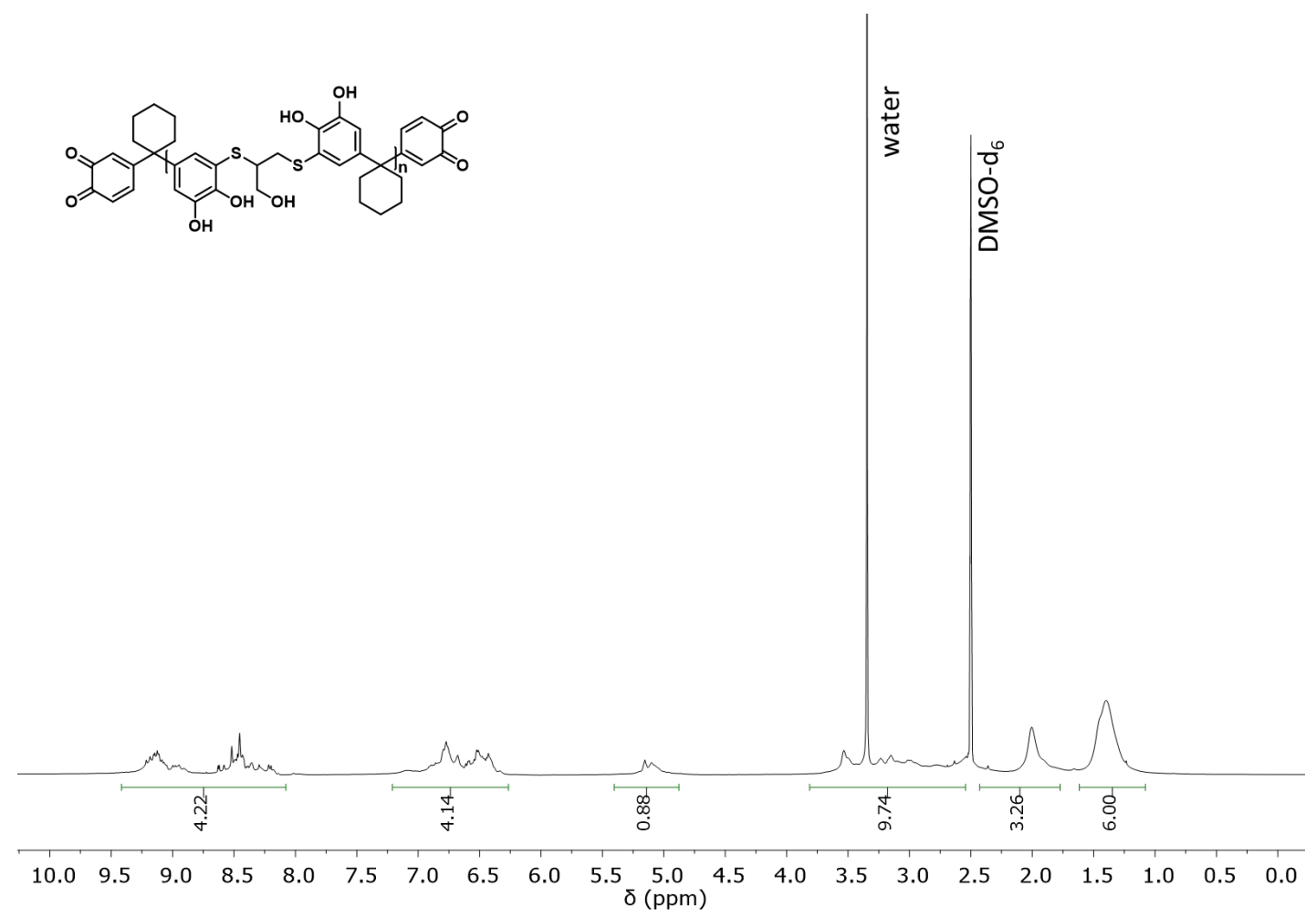

Figure S 76. ${ }^{1} \mathrm{H}-\mathrm{NMR}$-spectra of $\mathrm{p}(\mathrm{DCZ}-\mathrm{DMP})$ in DMSO-d ${ }_{6}$. 


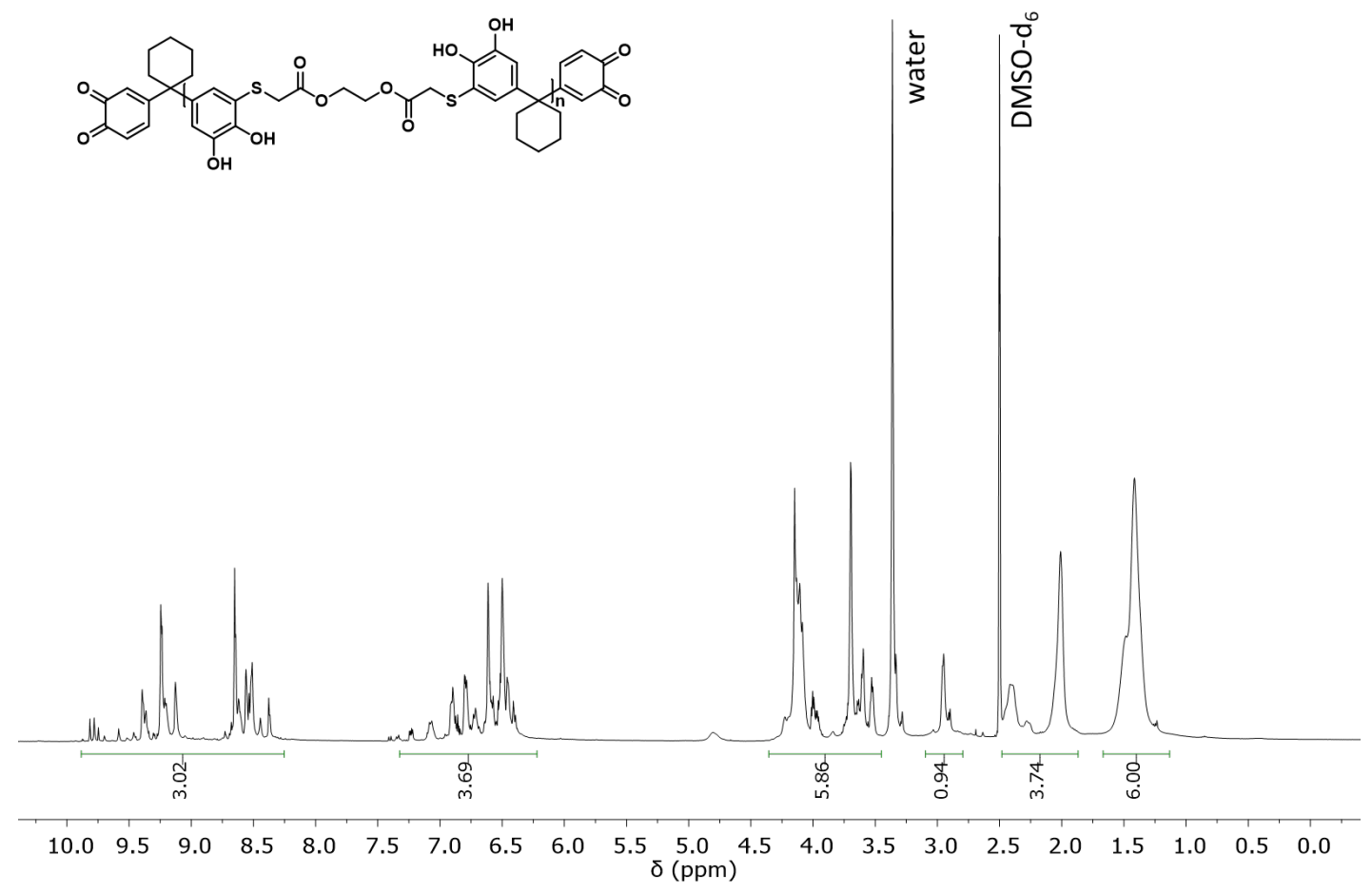

Figure S 77. ${ }^{1} \mathrm{H}-\mathrm{NMR}$-spectra of $\mathrm{p}(\mathrm{DCZ}-\mathrm{GDMA})$ in DMSO-d ${ }_{6}$.

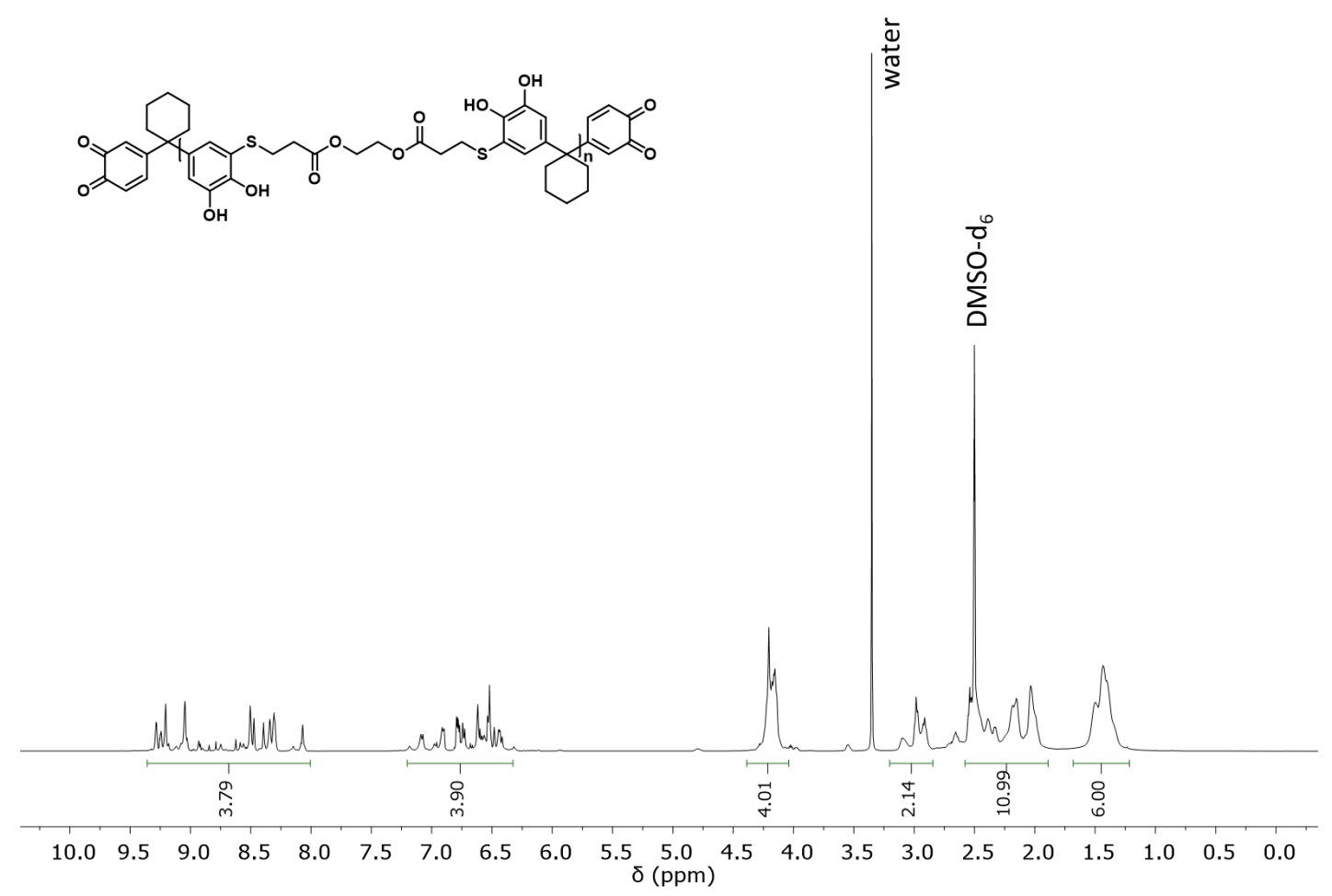

Figure $\mathrm{S} 78 .{ }^{1} \mathrm{H}-\mathrm{NMR}$-spectra of $\mathrm{p}(\mathrm{DCZ}-\mathrm{GDMP})$ in DMSO-d $\mathrm{d}_{6}$. 


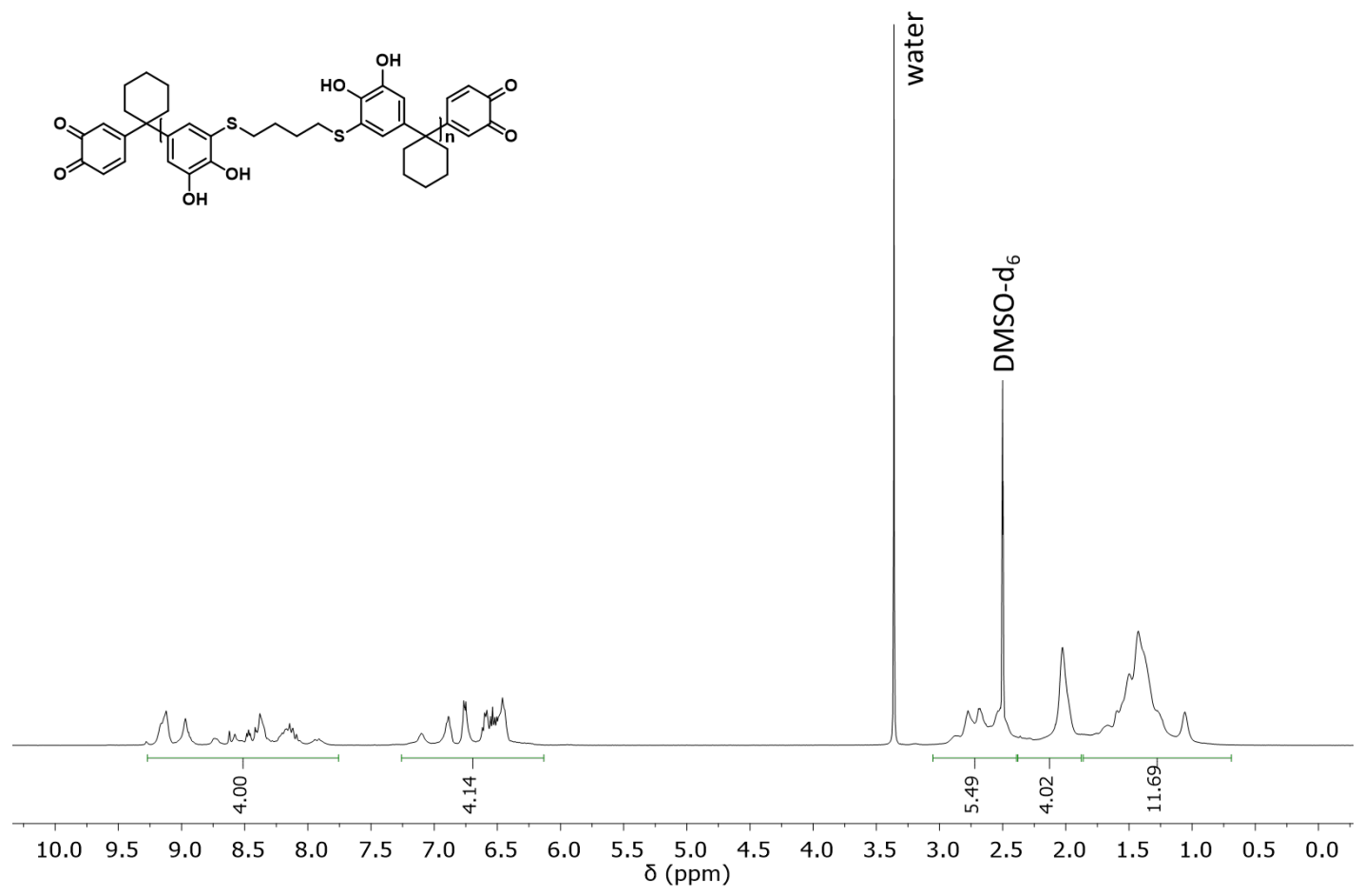

Figure S 79. ${ }^{1} \mathrm{H}-\mathrm{NMR}$-spectra of $\mathrm{p}(\mathrm{DCZ}-\mathrm{BDT})$ in DMSO-d $\mathrm{d}_{6}$.
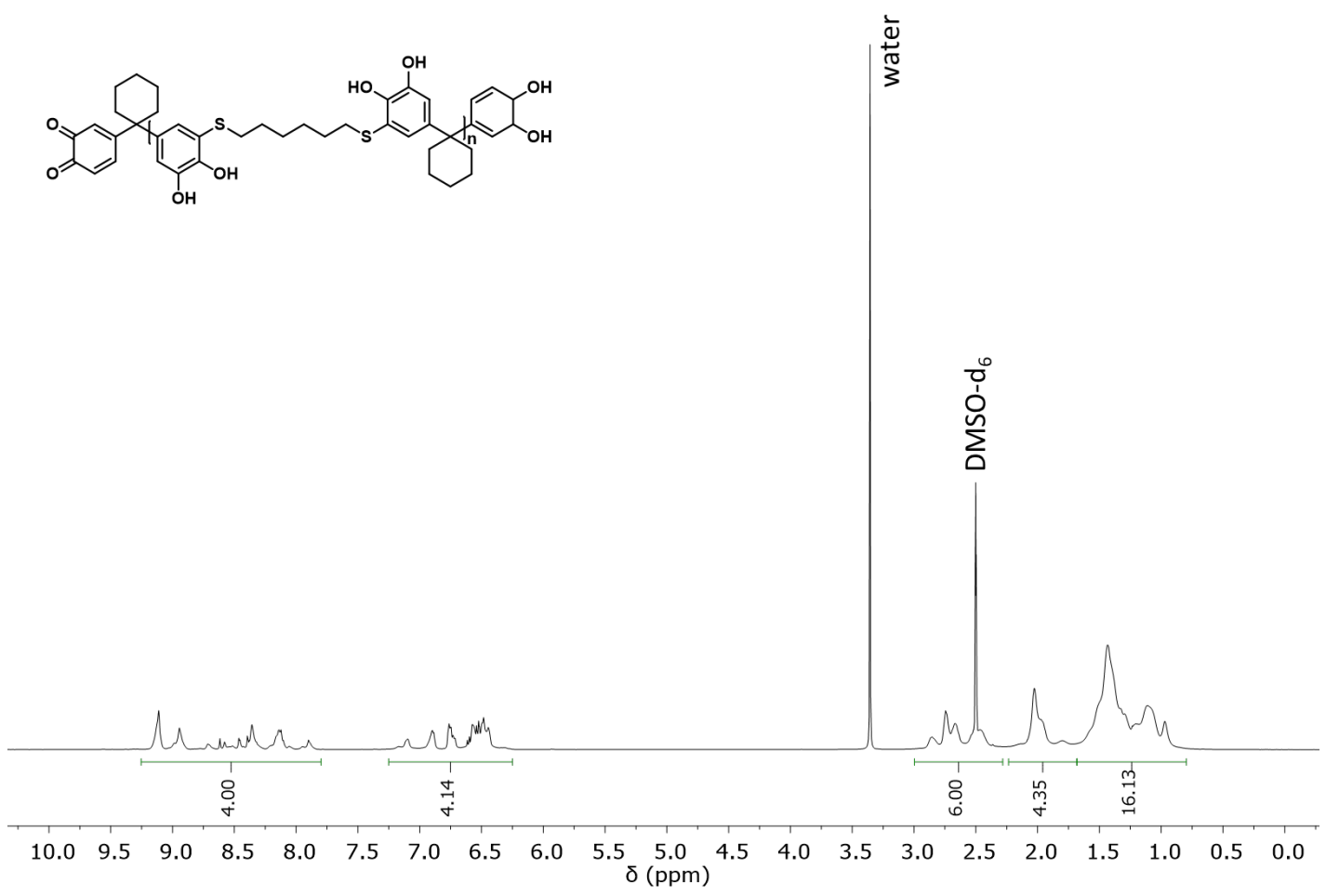

Figure S 80. ${ }^{1} \mathrm{H}-\mathrm{NMR}$-spectra of $\mathrm{p}(\mathrm{DCZ}-\mathrm{HDT})$ in $\mathrm{DMSO}^{-} \mathrm{d}_{6}$.

S 90 


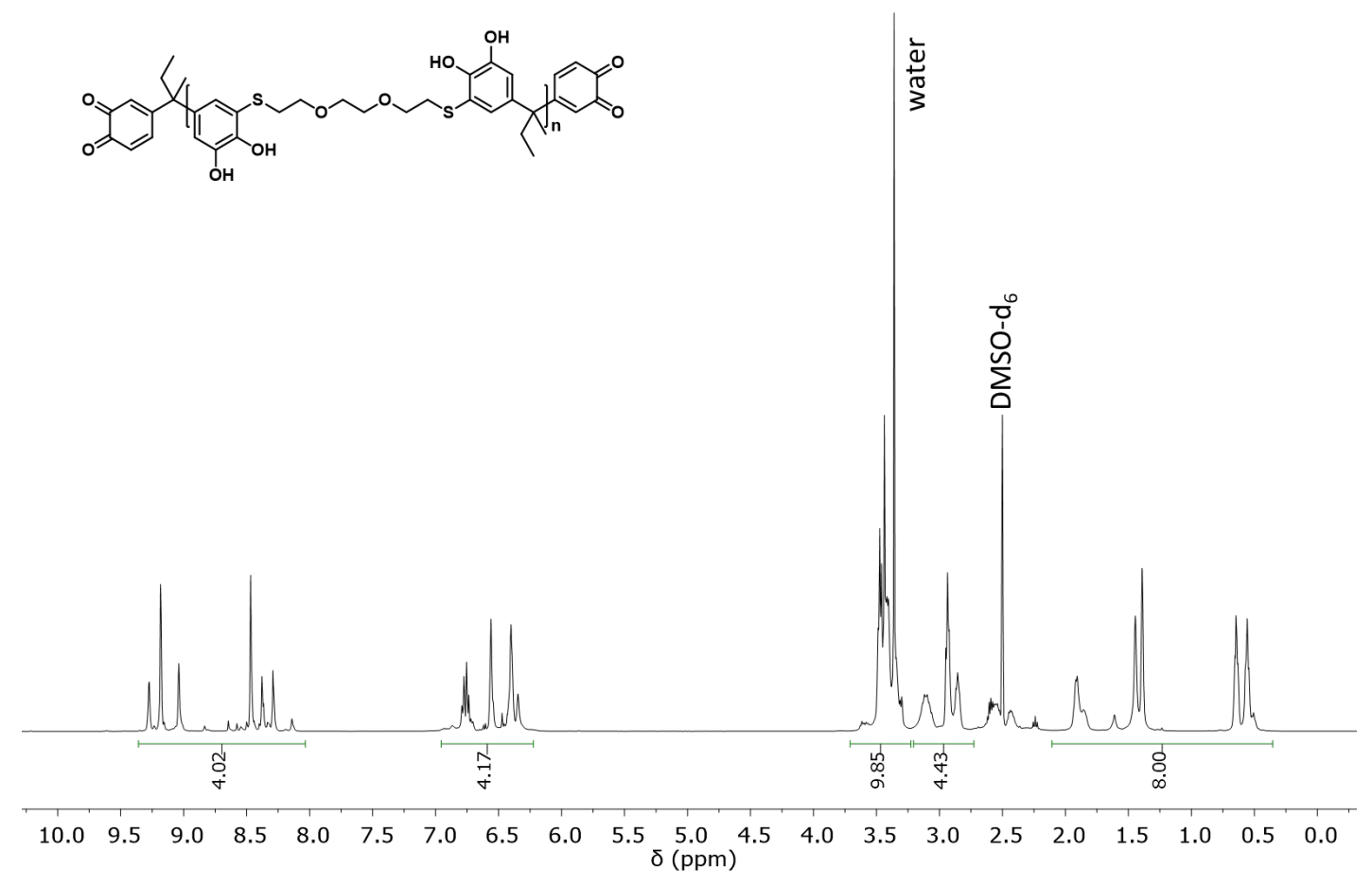

Figure S 81. ${ }^{1} \mathrm{H}-\mathrm{NMR}$-spectra of $\mathrm{p}(\mathrm{DCB}-\mathrm{EDET})$ in DMSO-d ${ }_{6}$.

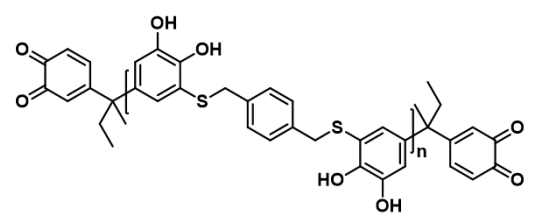

$\sum_{0}^{00}$

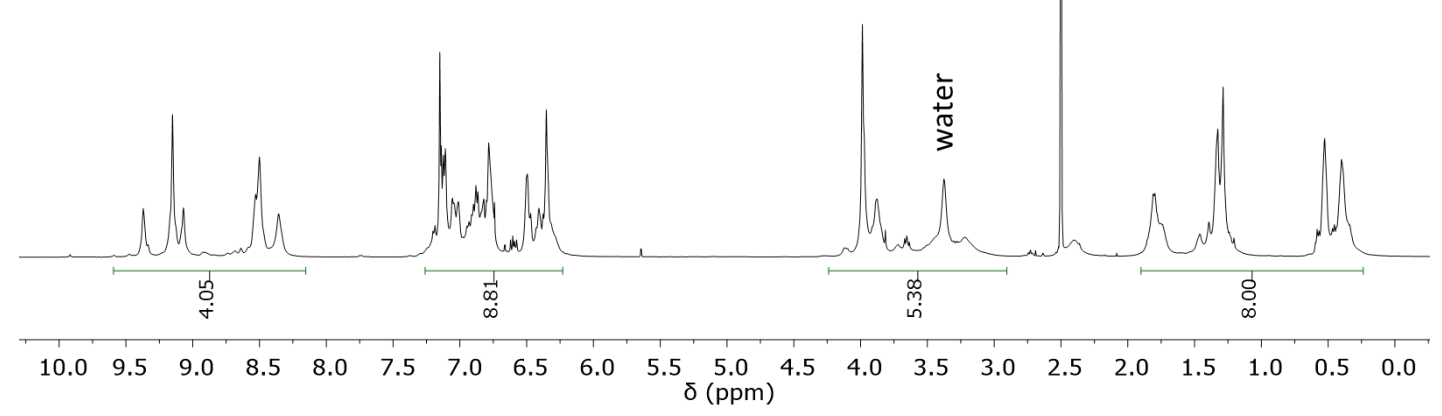

Figure S 82. ${ }^{1} \mathrm{H}-\mathrm{NMR}$-spectra of $\mathrm{p}(\mathrm{DCB}-\mathrm{BMT})$ in DMSO-d $\mathrm{d}_{6}$. 


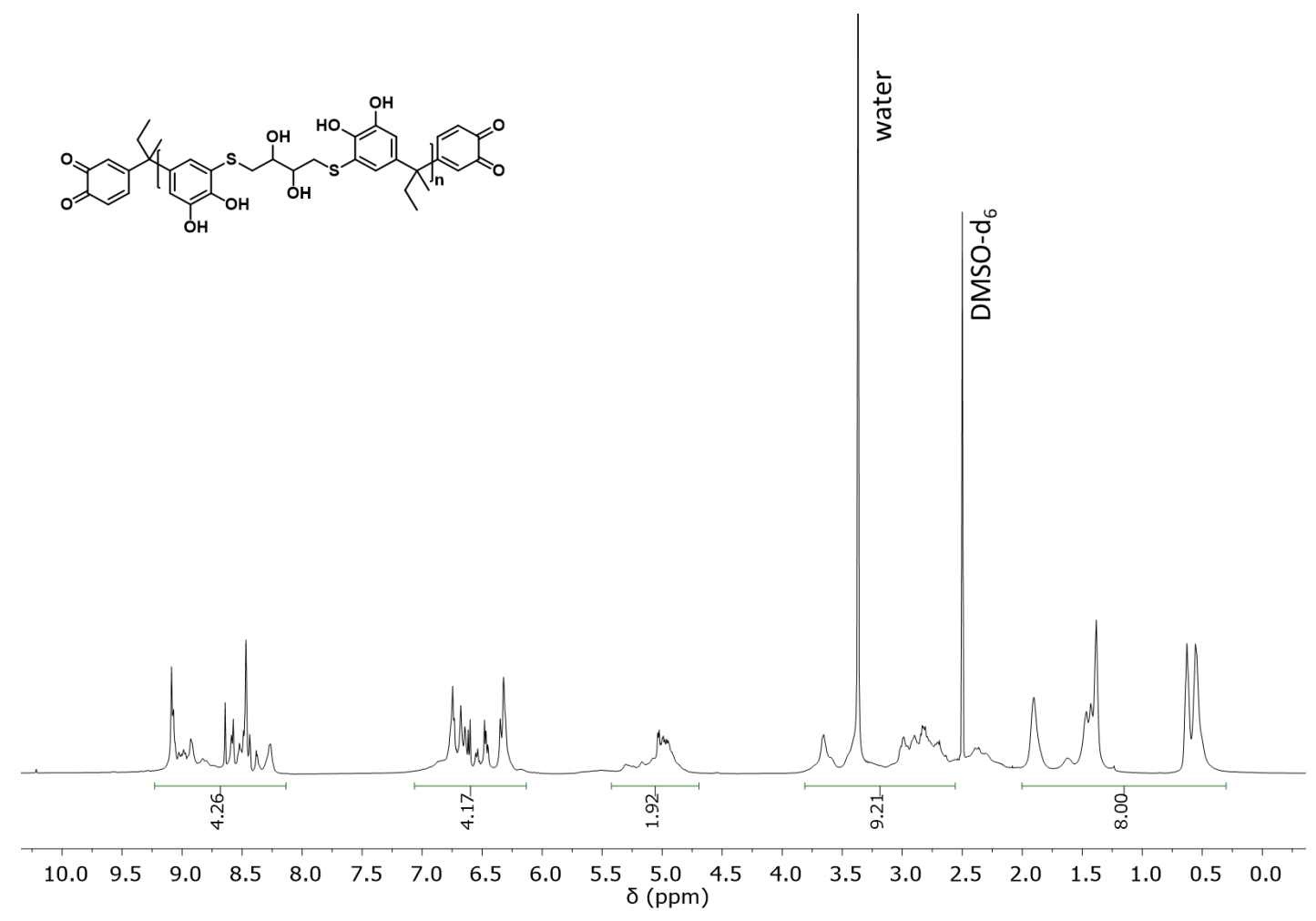

Figure S 83. ${ }^{1} \mathrm{H}-\mathrm{NMR}$-spectra of $\mathrm{p}(\mathrm{DCB}-\mathrm{DTT})$ in $\mathrm{DMSO}-\mathrm{d}_{6}$.

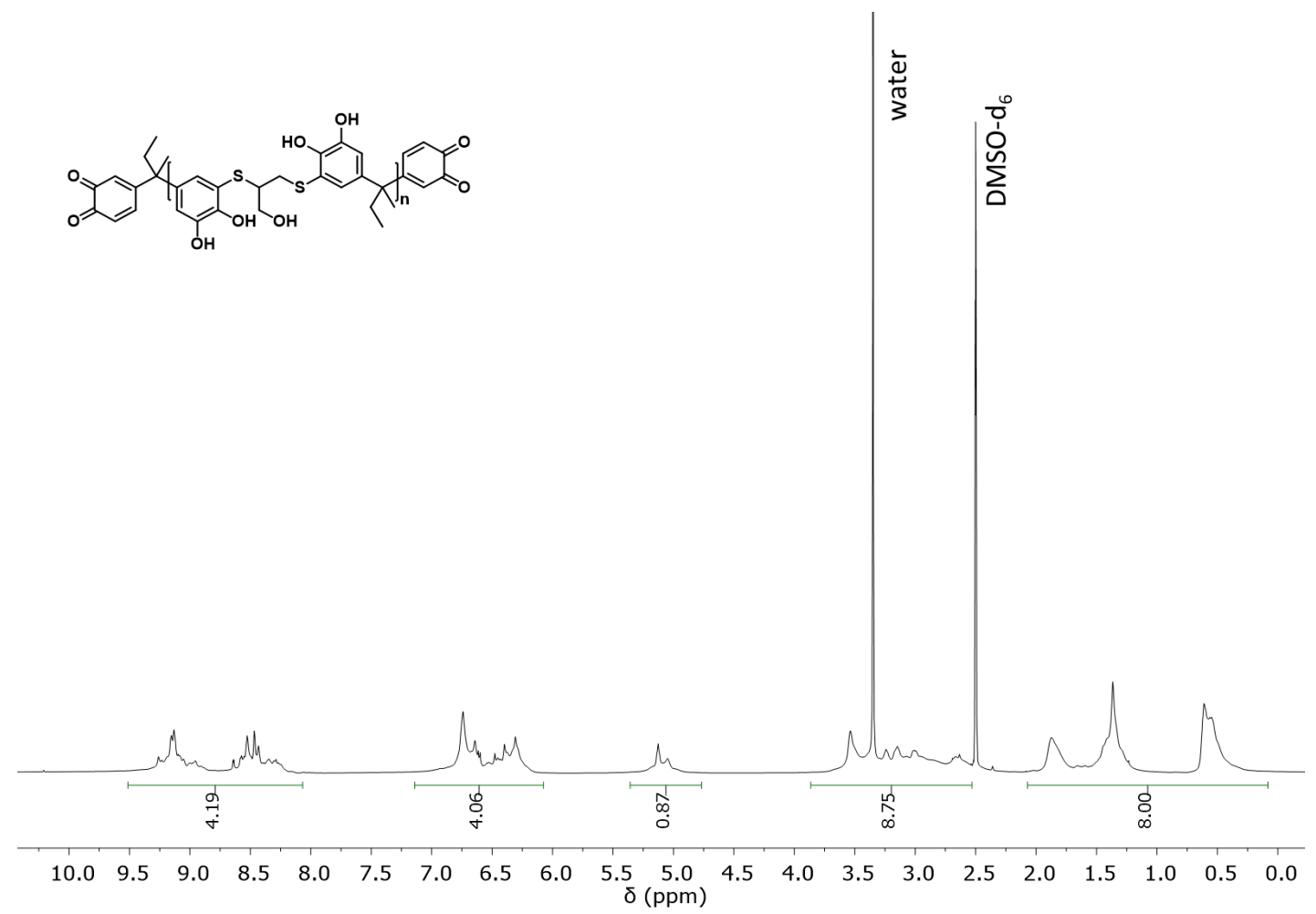

Figure S 84. ${ }^{1} \mathrm{H}-\mathrm{NMR}$-spectra of $\mathrm{p}(\mathrm{DCB}-\mathrm{DMP})$ in DMSO-d $\mathrm{d}_{6}$. 

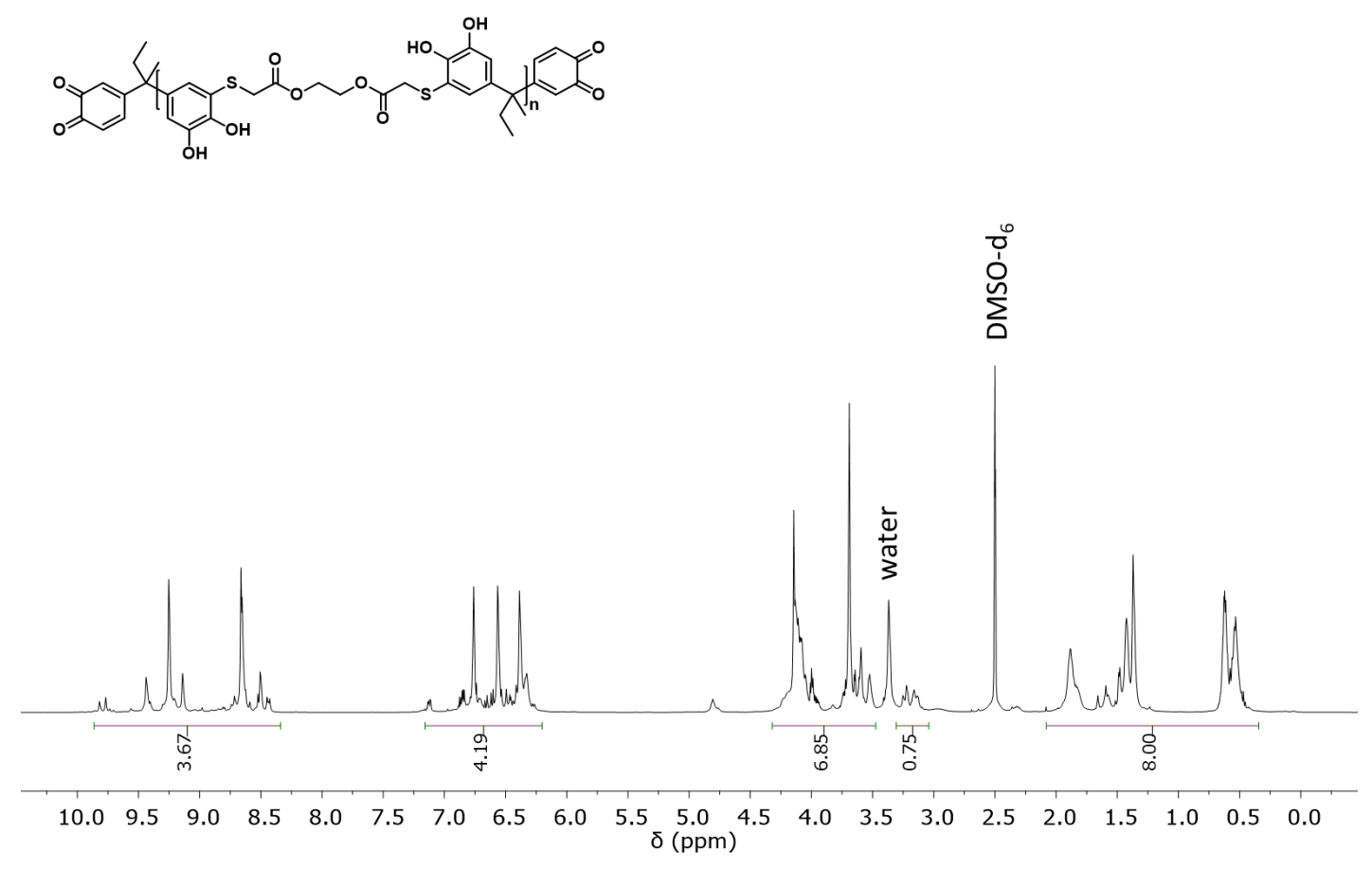

Figure S 85. ${ }^{1} \mathrm{H}-\mathrm{NMR}$-spectra of $\mathrm{p}(\mathrm{DCB}-\mathrm{GDMA})$ in DMSO-d $\mathrm{d}_{6}$.

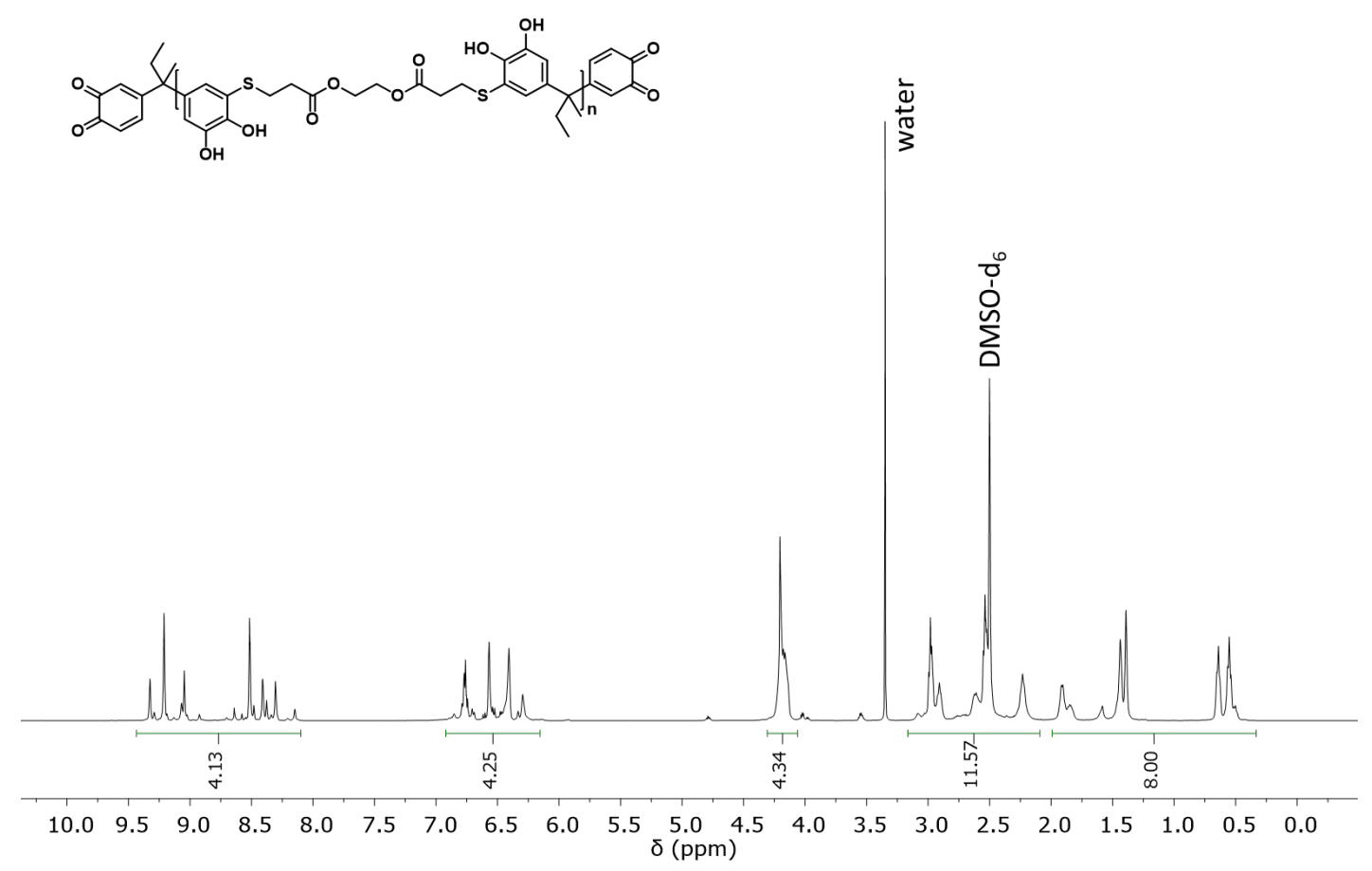

Figure S 86. ${ }^{1} \mathrm{H}-\mathrm{NMR}$-spectra of $\mathrm{p}(\mathrm{DCB}-\mathrm{GDMP})$ in DMSO-d $\mathrm{d}_{6}$. 


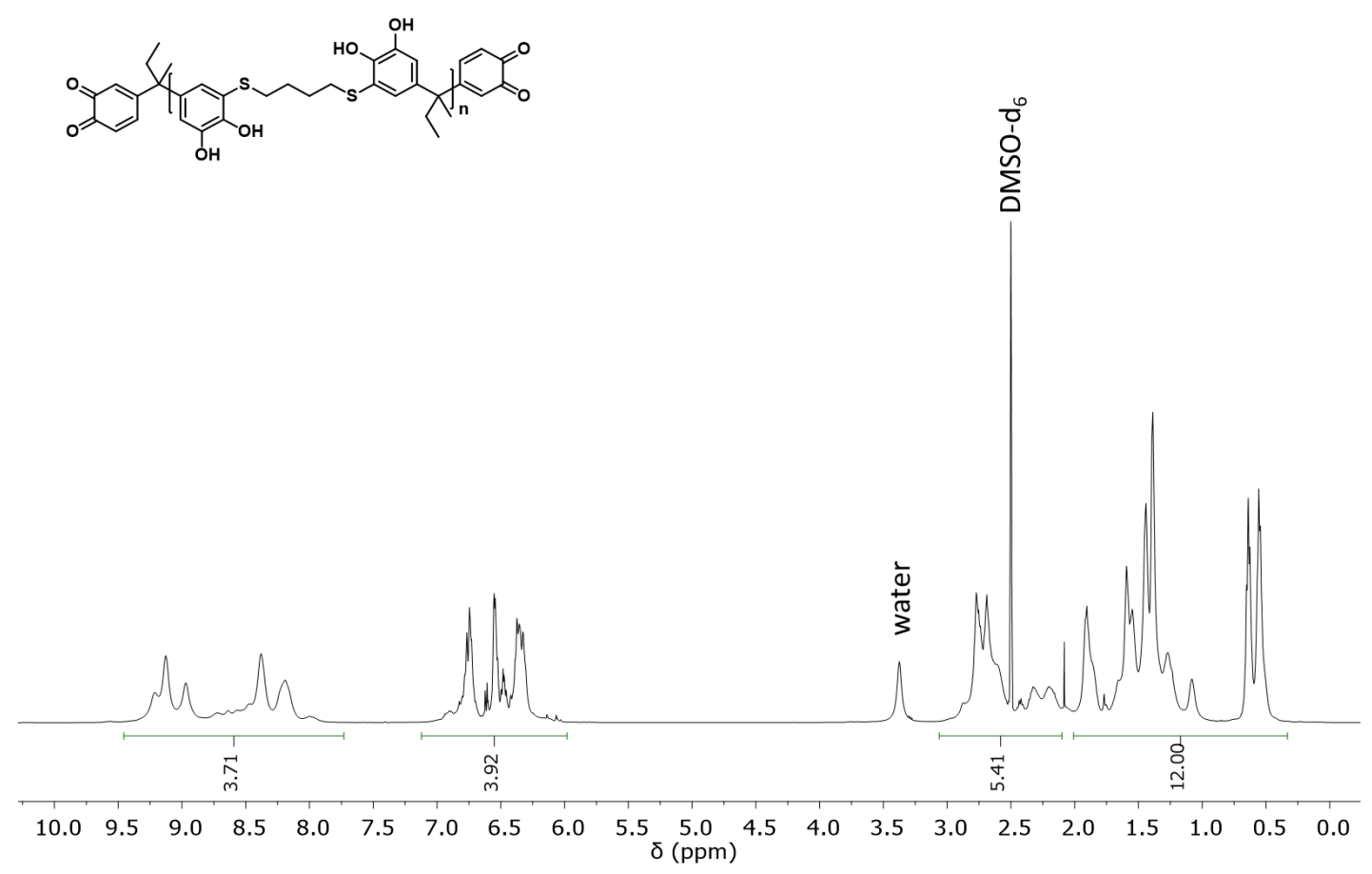

Figure S 87. ${ }^{1} \mathrm{H}-\mathrm{NMR}$-spectra of $\mathrm{p}(\mathrm{DCB}-\mathrm{BDT})$ in DMSO-d ${ }_{6}$.

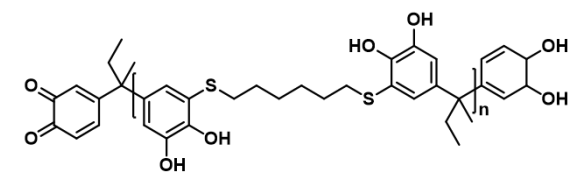

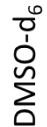

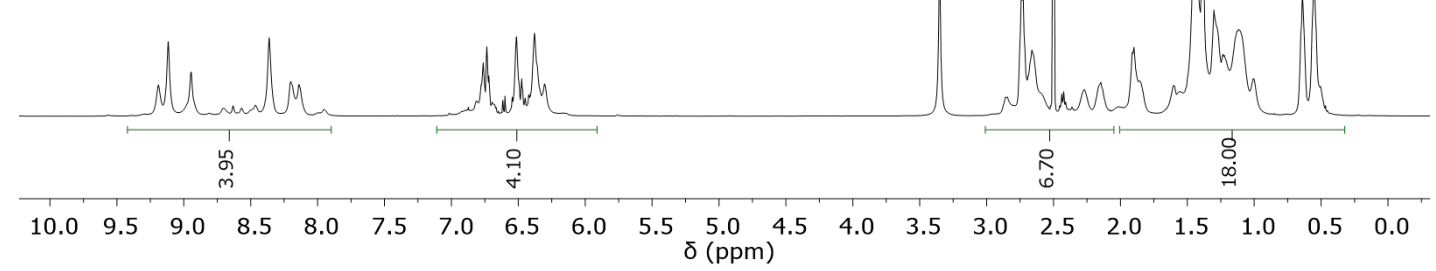

Figure S 88. ${ }^{1} \mathrm{H}-\mathrm{NMR}$-spectra of $\mathrm{p}(\mathrm{DCB}-\mathrm{HDT})$ in DMSO-d $\mathrm{d}_{6}$. 


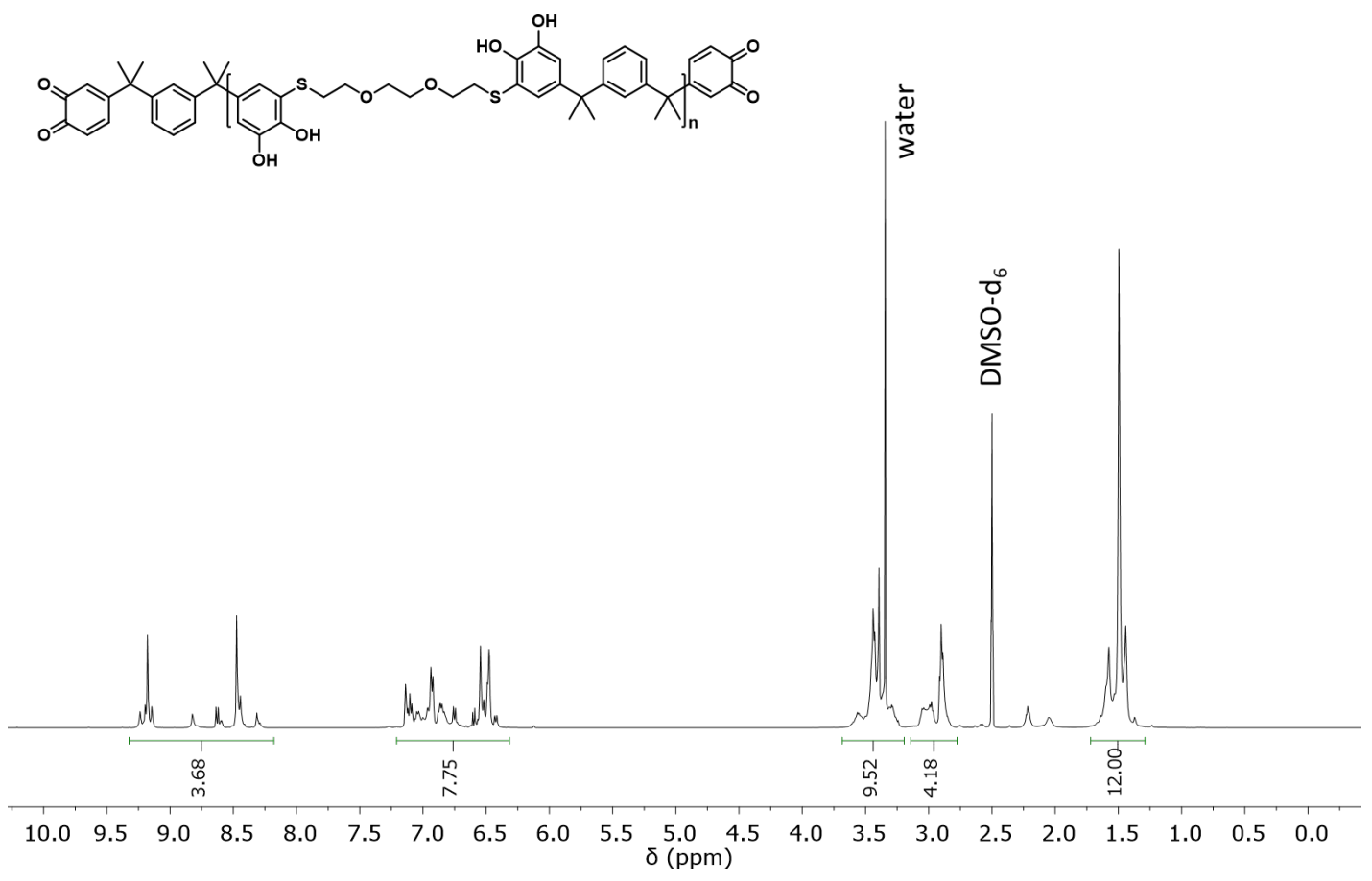

Figure S 89. ${ }^{1} \mathrm{H}-\mathrm{NMR}-$ spectra of $\mathrm{p}(\mathrm{DCM}-\mathrm{EDET})$ in DMSO-d $\mathrm{d}_{6}$.
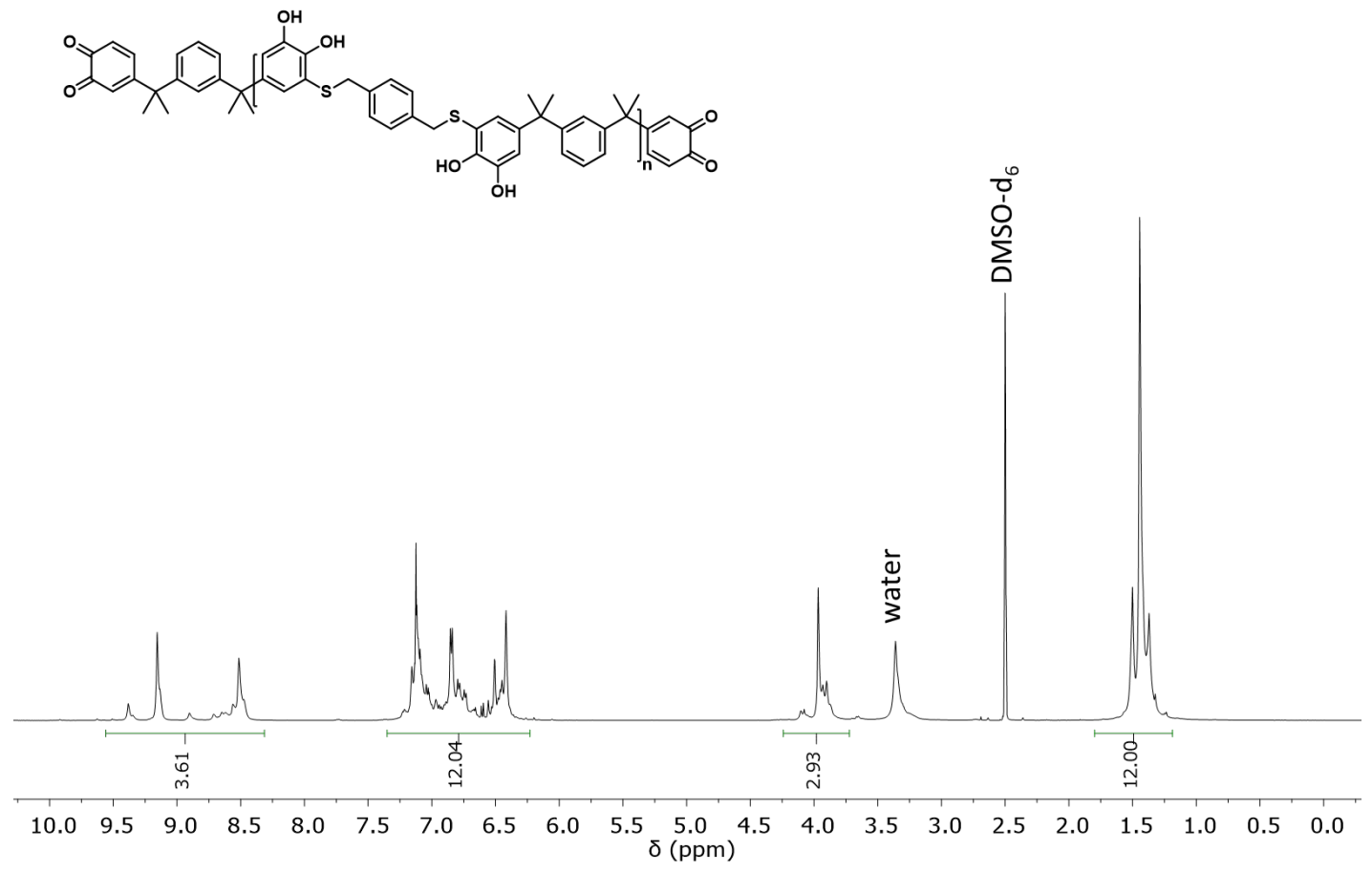

Figure S 90. ${ }^{1} \mathrm{H}-\mathrm{NMR}$-spectra of $\mathrm{p}(\mathrm{DCM}-\mathrm{BMT})$ in DMSO-d $\mathrm{d}_{6}$.

S 95 


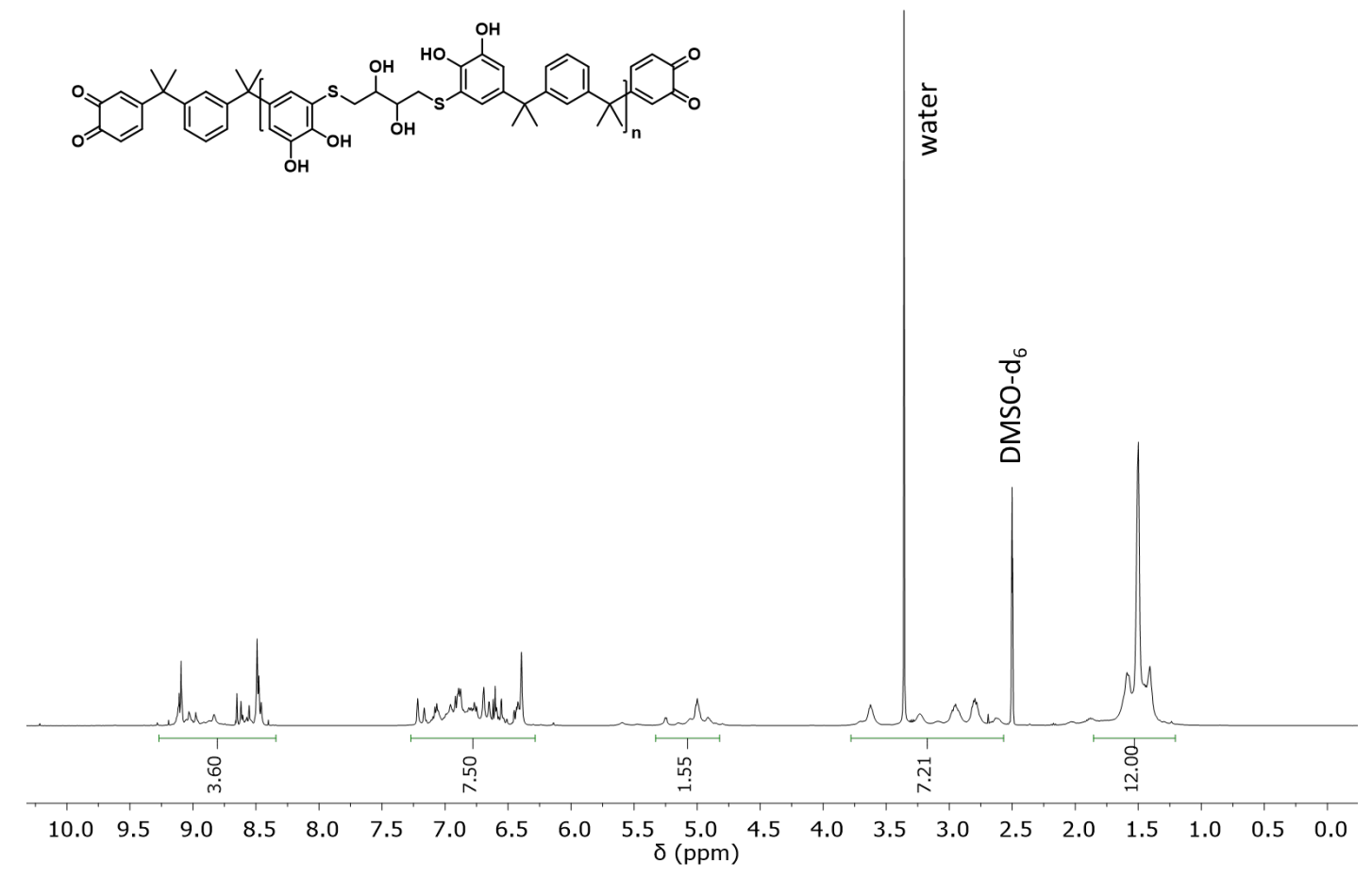

Figure S 91. ${ }^{1} \mathrm{H}-\mathrm{NMR}$-spectra of $\mathrm{p}(\mathrm{DCM}-\mathrm{DTT})$ in DMSO-d 6 .

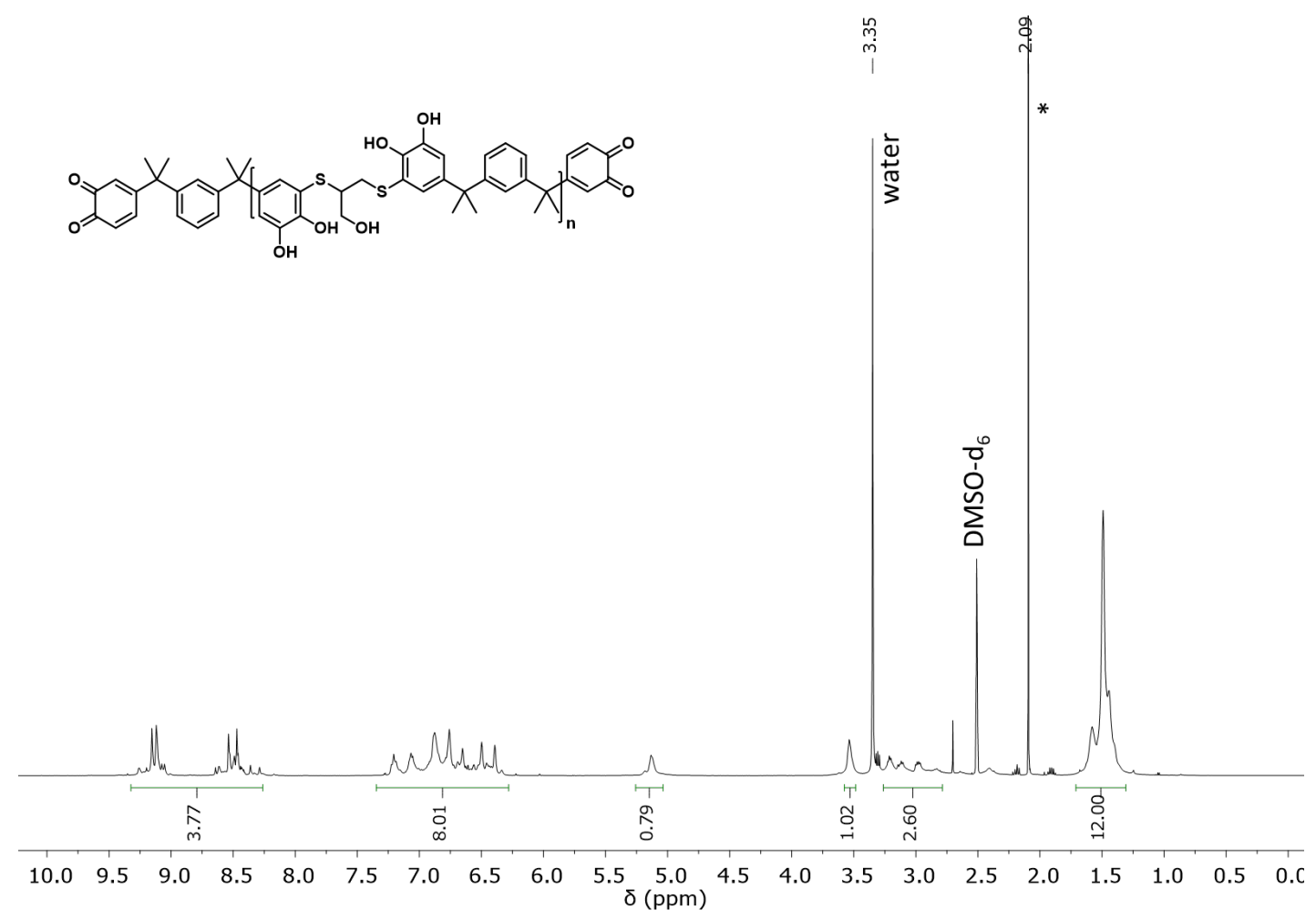

Figure S 92. ${ }^{1} \mathrm{H}-\mathrm{NMR}$-spectra of $\mathrm{p}(\mathrm{DCM}-\mathrm{DMP})$ in DMSO-d $\mathrm{d}_{6} \cdot{ }^{*}$ Residual acetone from precipitation procedure. 


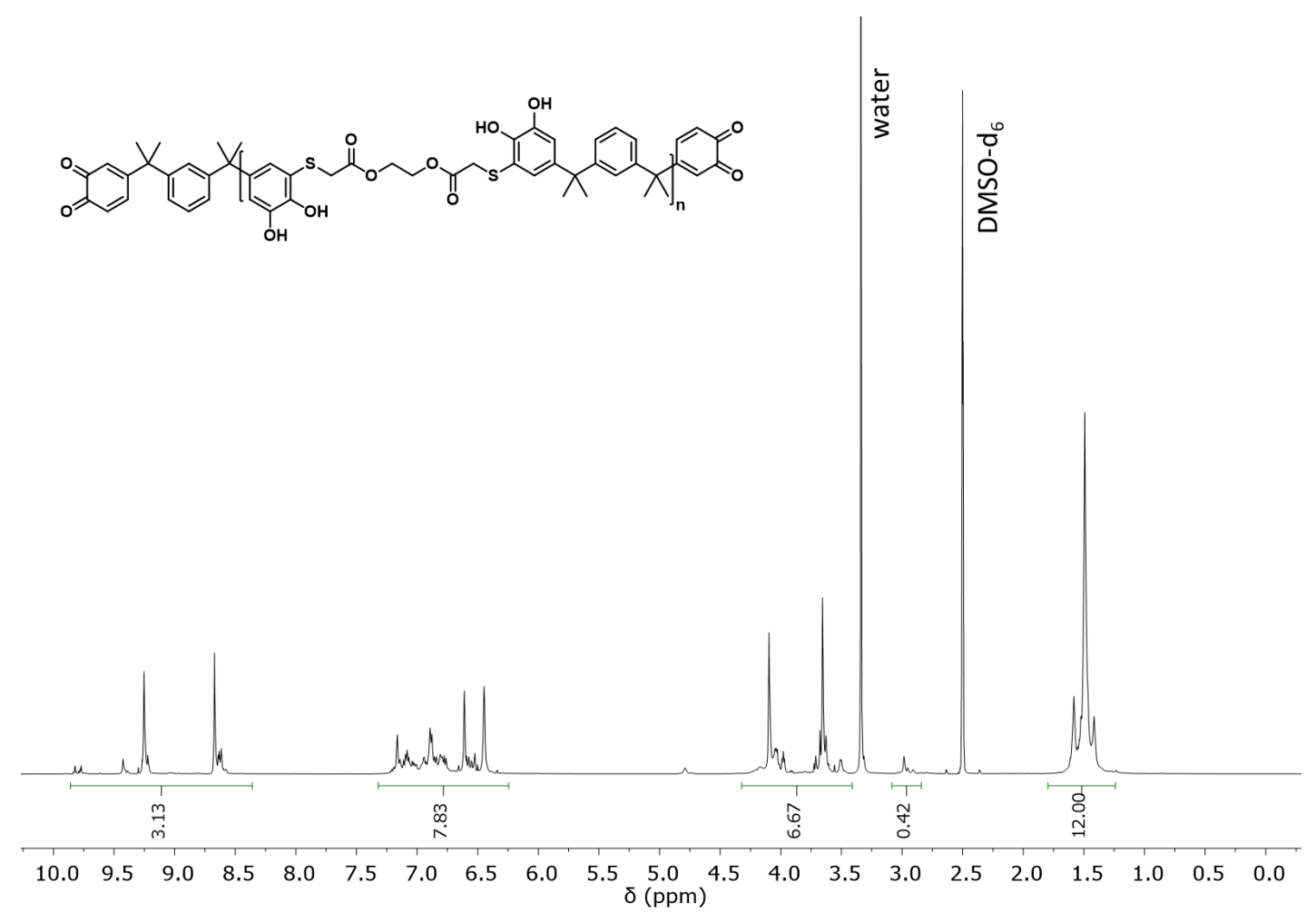

Figure S 93. ${ }^{1} \mathrm{H}-\mathrm{NMR}$-spectra of $\mathrm{p}(\mathrm{DCM}-\mathrm{GDMA})$ in DMSO-d $\mathrm{d}_{6}$.
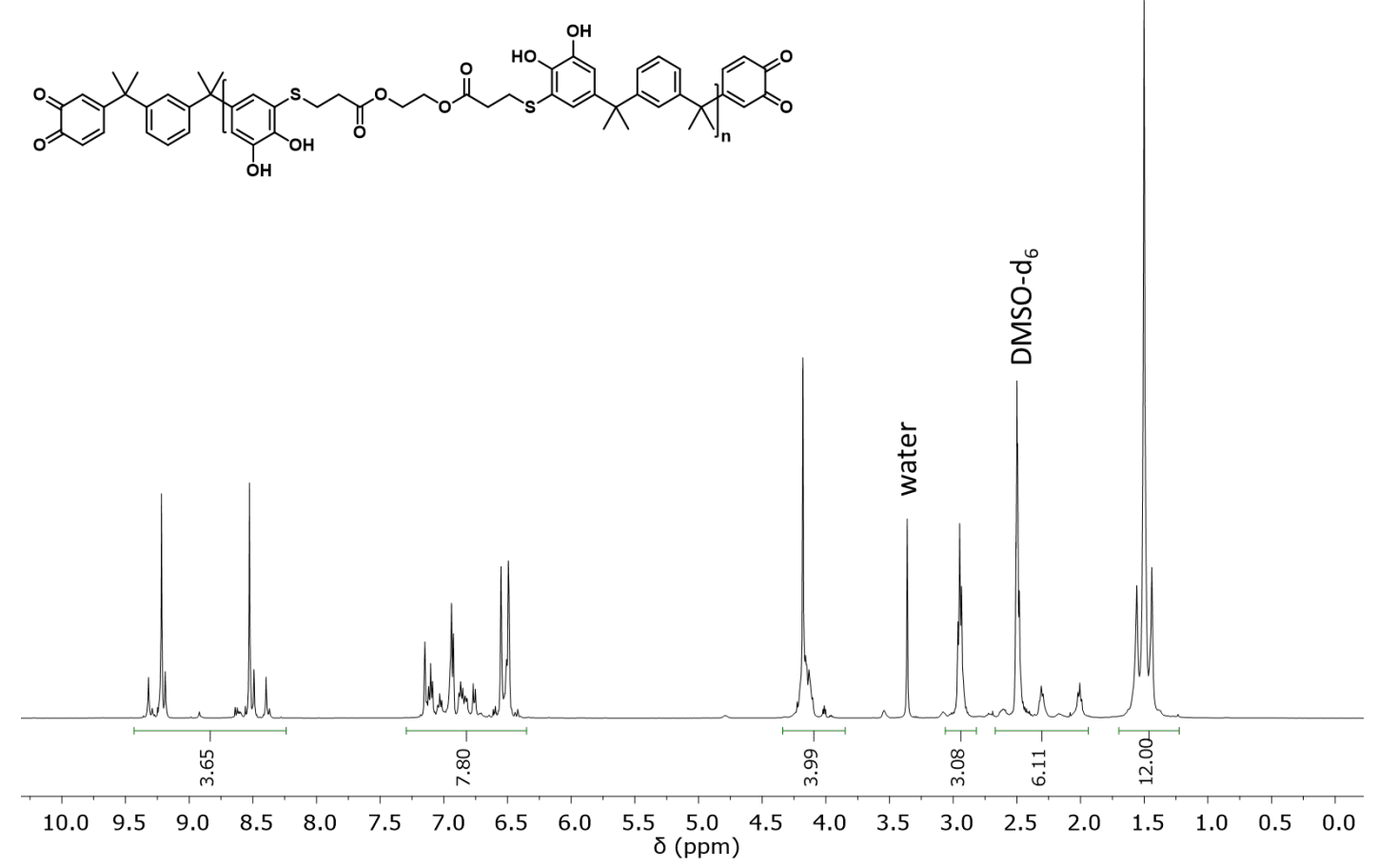

Figure S 94. ${ }^{1} \mathrm{H}-\mathrm{NMR}$-spectra of p(DCM-GDMP) in DMSO-d ${ }_{6}$. 


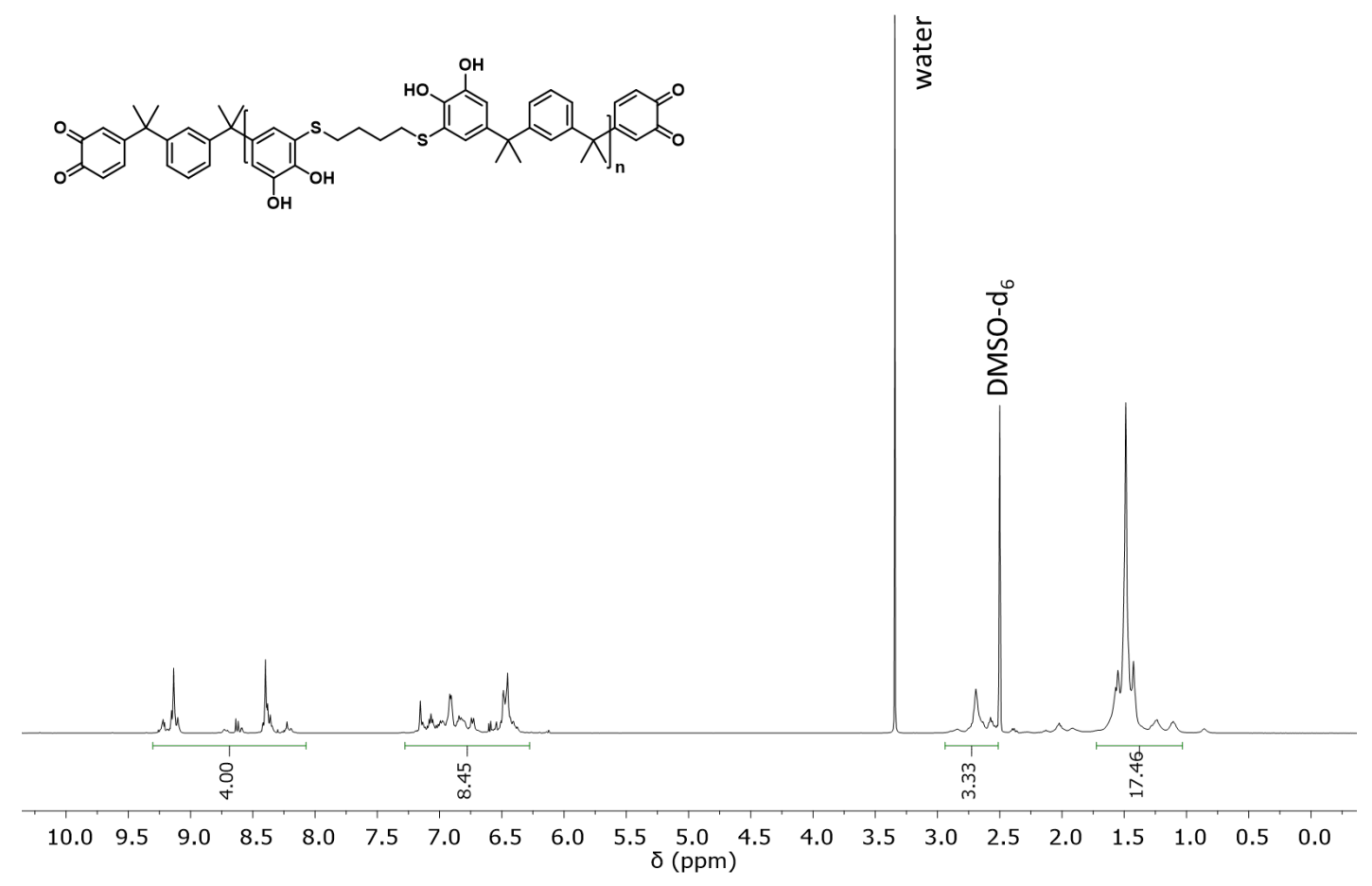

Figure S 95. ${ }^{1} \mathrm{H}-\mathrm{NMR}$-spectra of $\mathrm{p}(\mathrm{DCM}-\mathrm{BDT})$ in DMSO-d ${ }_{6}$.

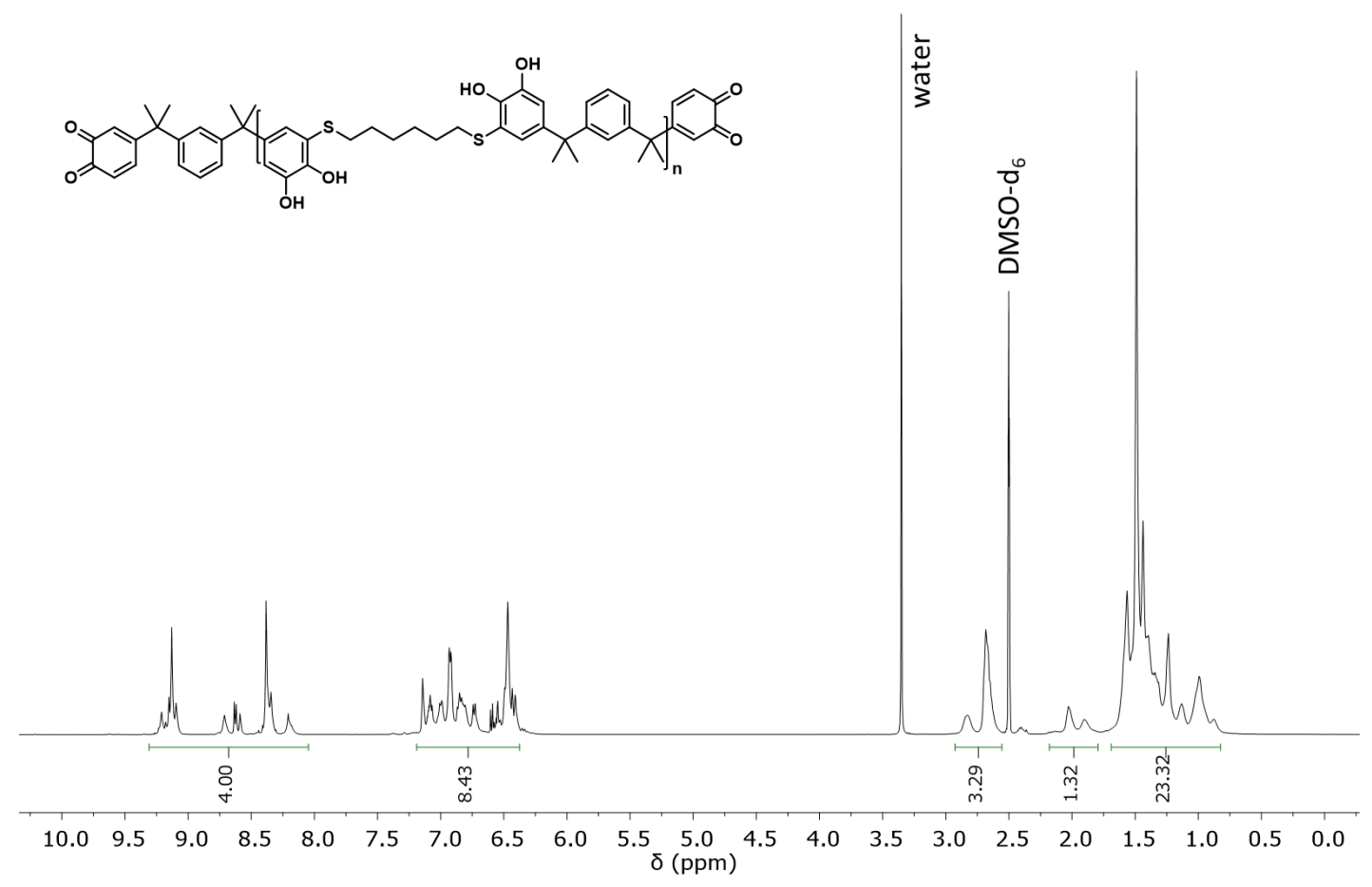

Figure S 96. ${ }^{1} \mathrm{H}-\mathrm{NMR}$-spectra of $\mathrm{p}(\mathrm{DCM}-\mathrm{HDT})$ in DMSO-d $\mathrm{d}_{6}$. 


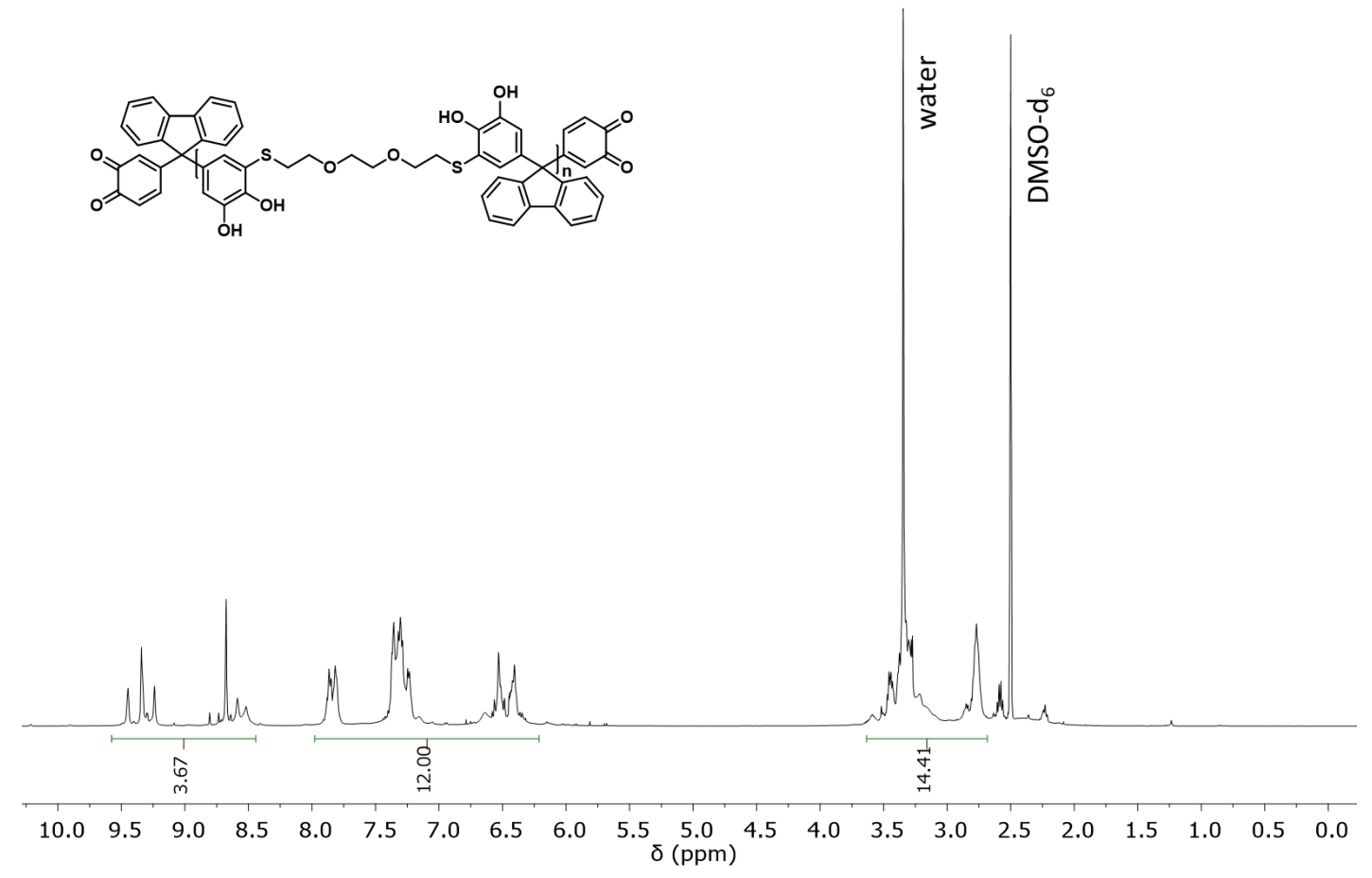

Figure S 97. ${ }^{1} \mathrm{H}-\mathrm{NMR}$-spectra of $\mathrm{p}$ (DCFL-EDET) in DMSO-d $\mathrm{d}_{6}$.
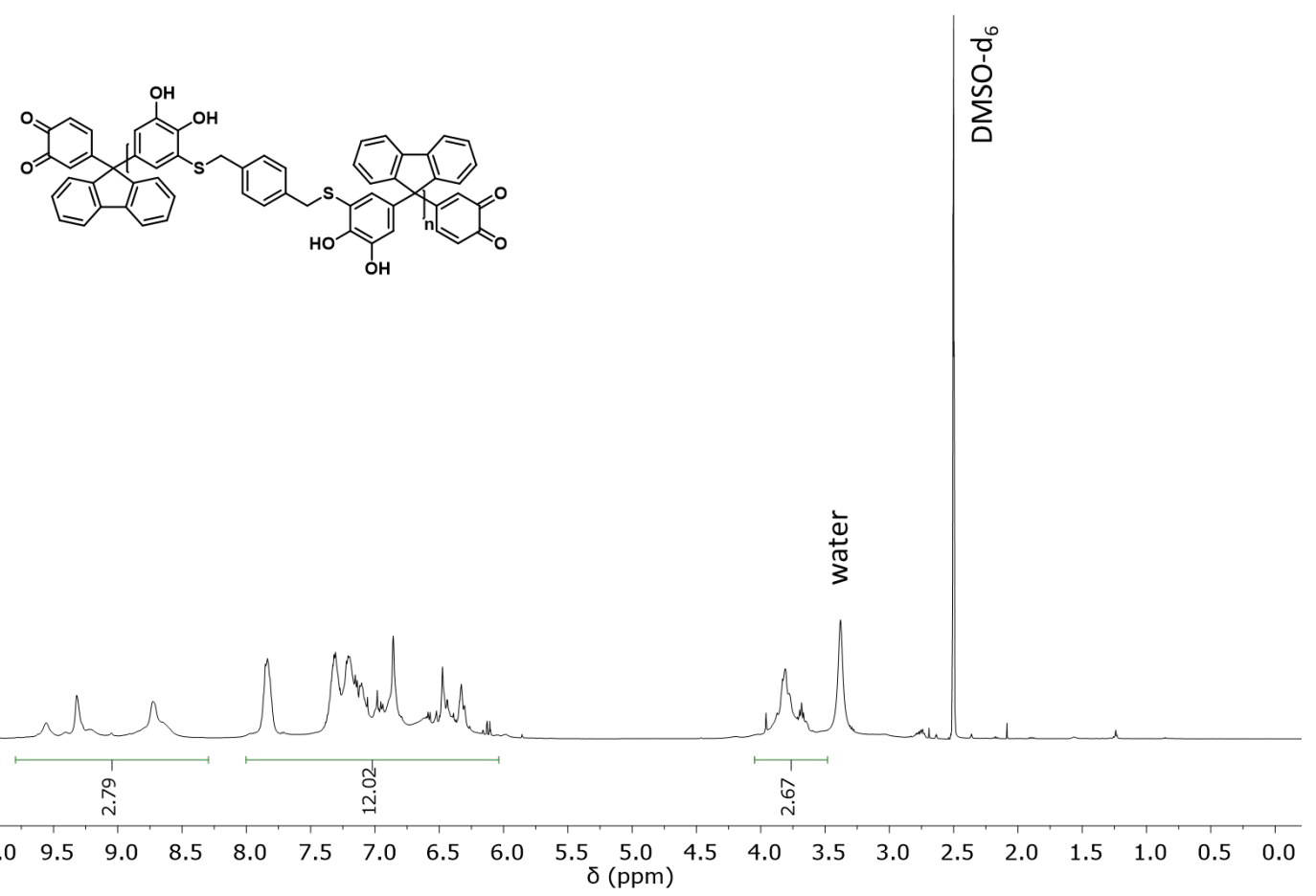

Figure S 98. ${ }^{1} \mathrm{H}-\mathrm{NMR}$-spectra of $\mathrm{p}$ (DCFL-BMT) in DMSO-d ${ }_{6}$. 


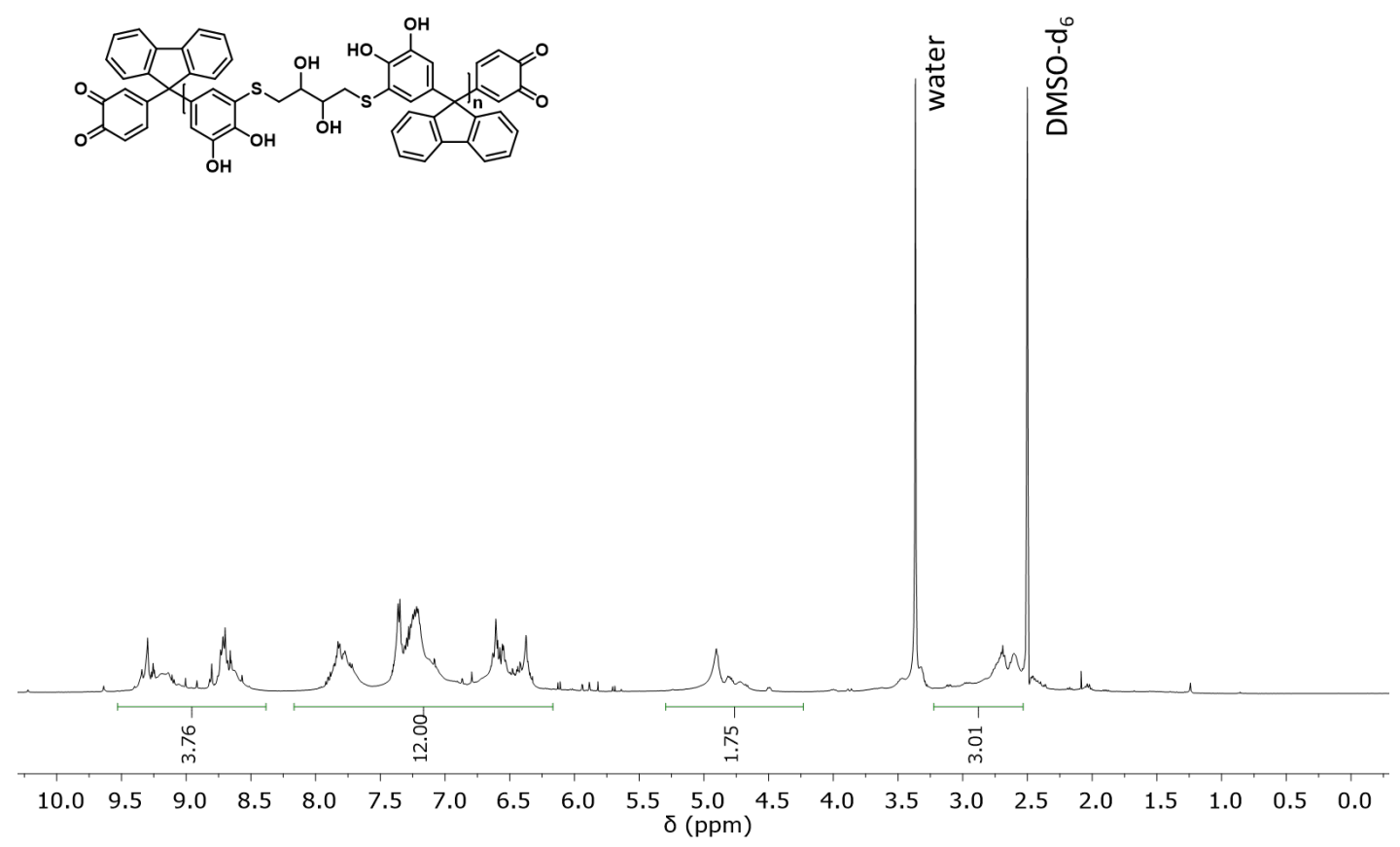

Figure S 99. ${ }^{1} \mathrm{H}-\mathrm{NMR}$-spectra of $\mathrm{p}(\mathrm{DCFL}-\mathrm{DTT})$ in DMSO-d $\mathrm{d}_{6}$.

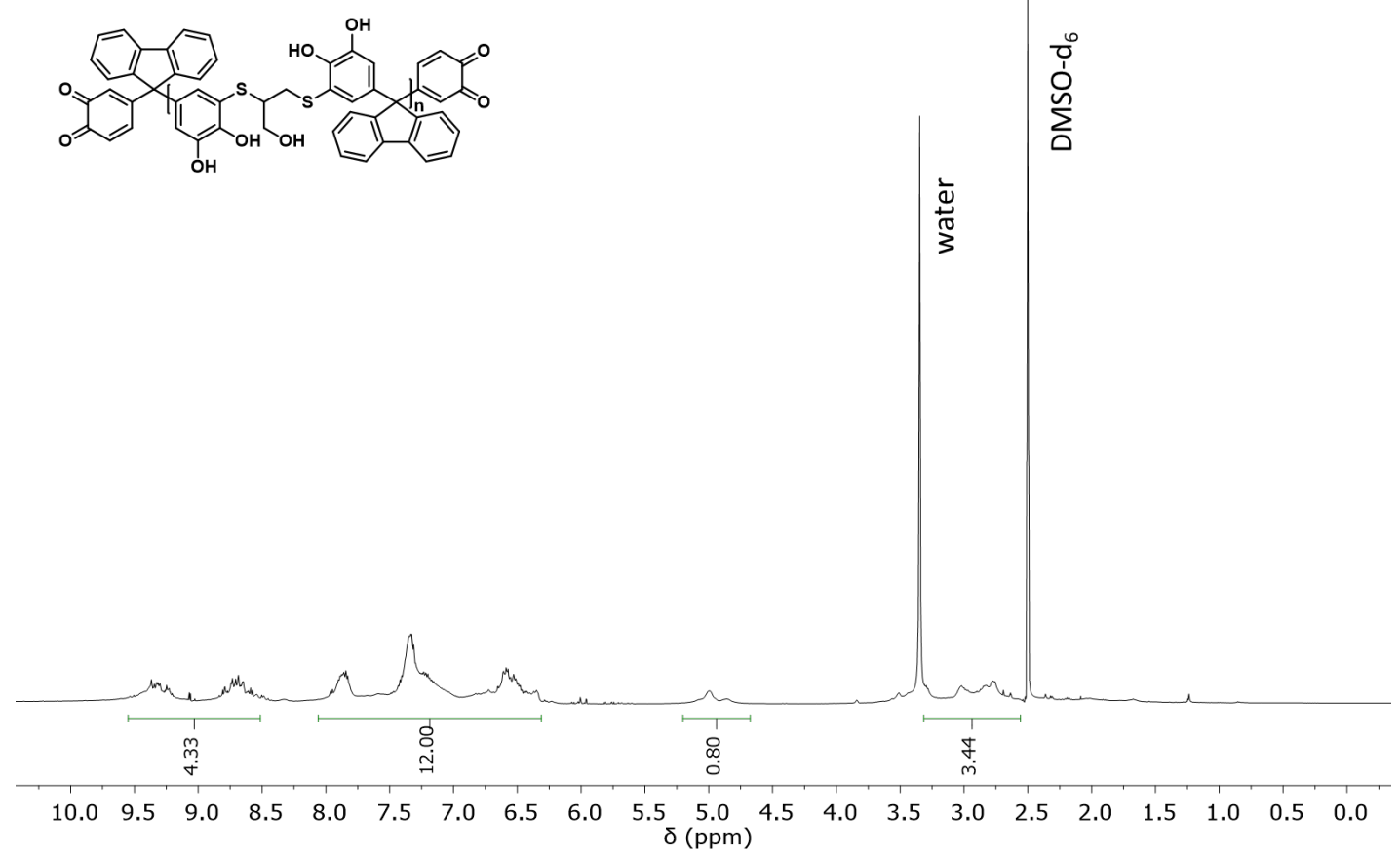

Figure S 100. ${ }^{1} \mathrm{H}-\mathrm{NMR}$-spectra of $\mathrm{p}$ (DCFL-DMP) in DMSO-d ${ }_{6}$. 

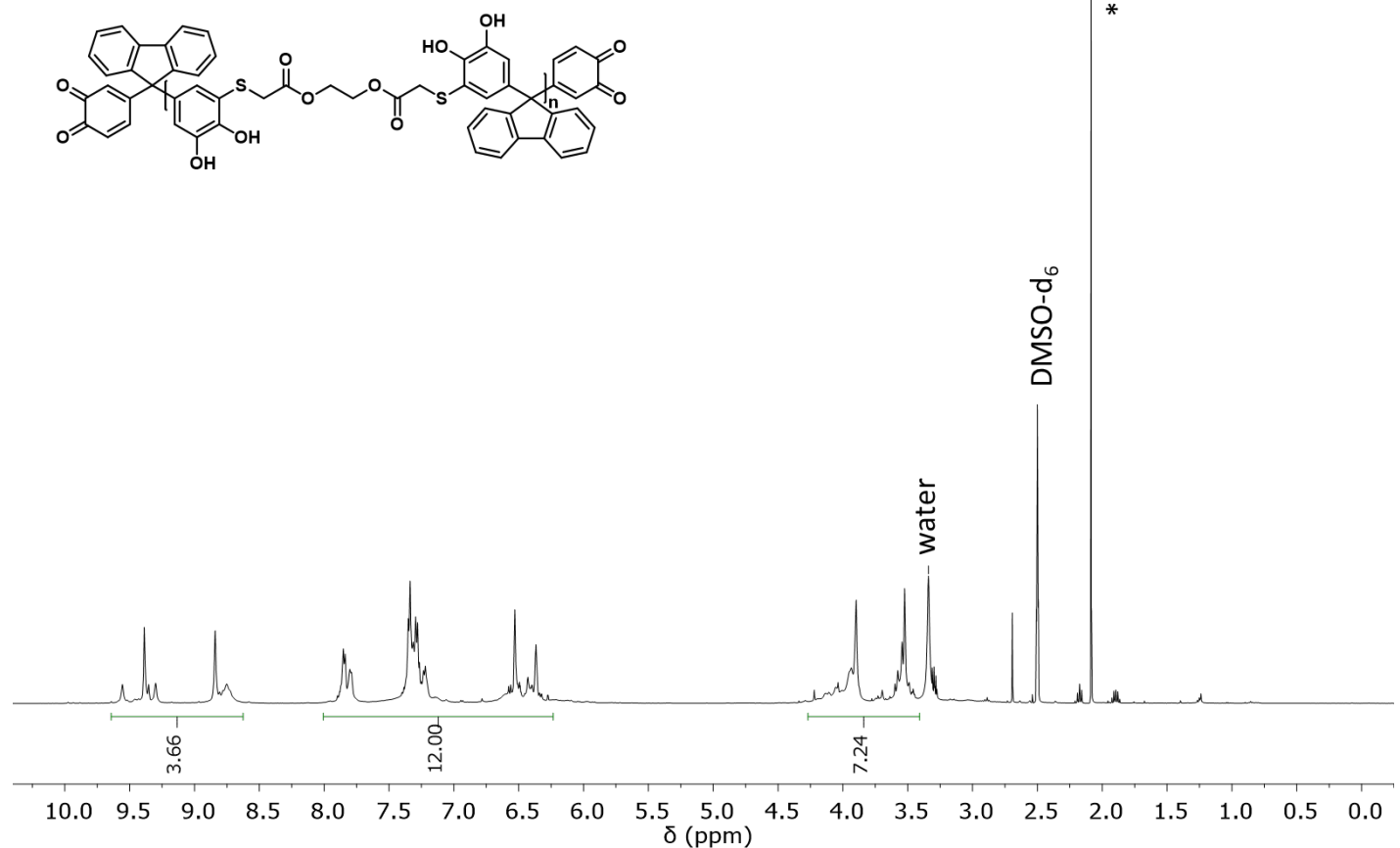

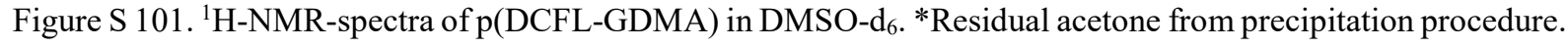
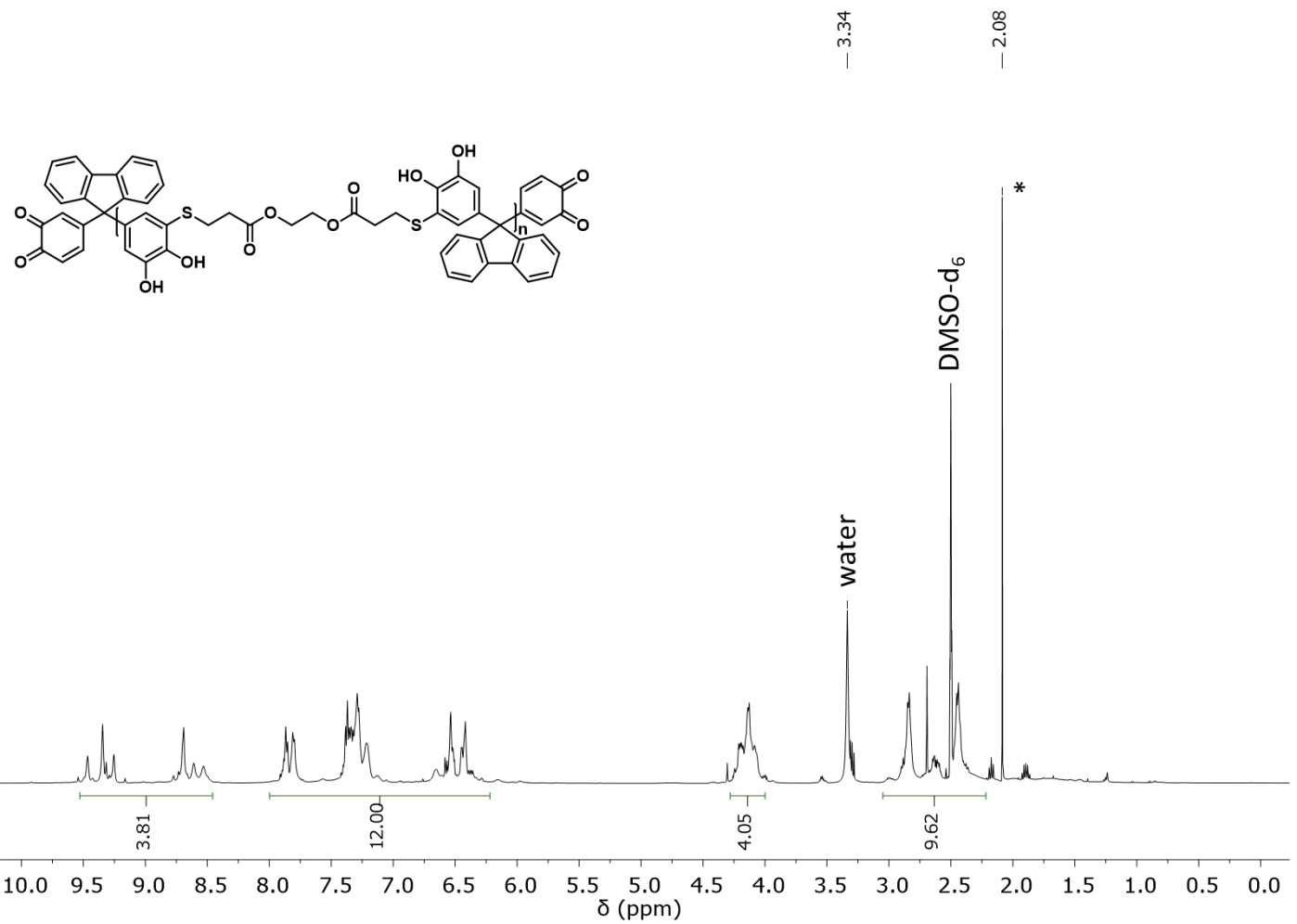

Figure S 102. ${ }^{1} \mathrm{H}-\mathrm{NMR}$-spectra of $\mathrm{p}(\mathrm{DCFL}-\mathrm{GDMP})$ in DMSO- $\mathrm{d}_{6} .{ }^{*}$ Residual acetone from precipitation procedure. 


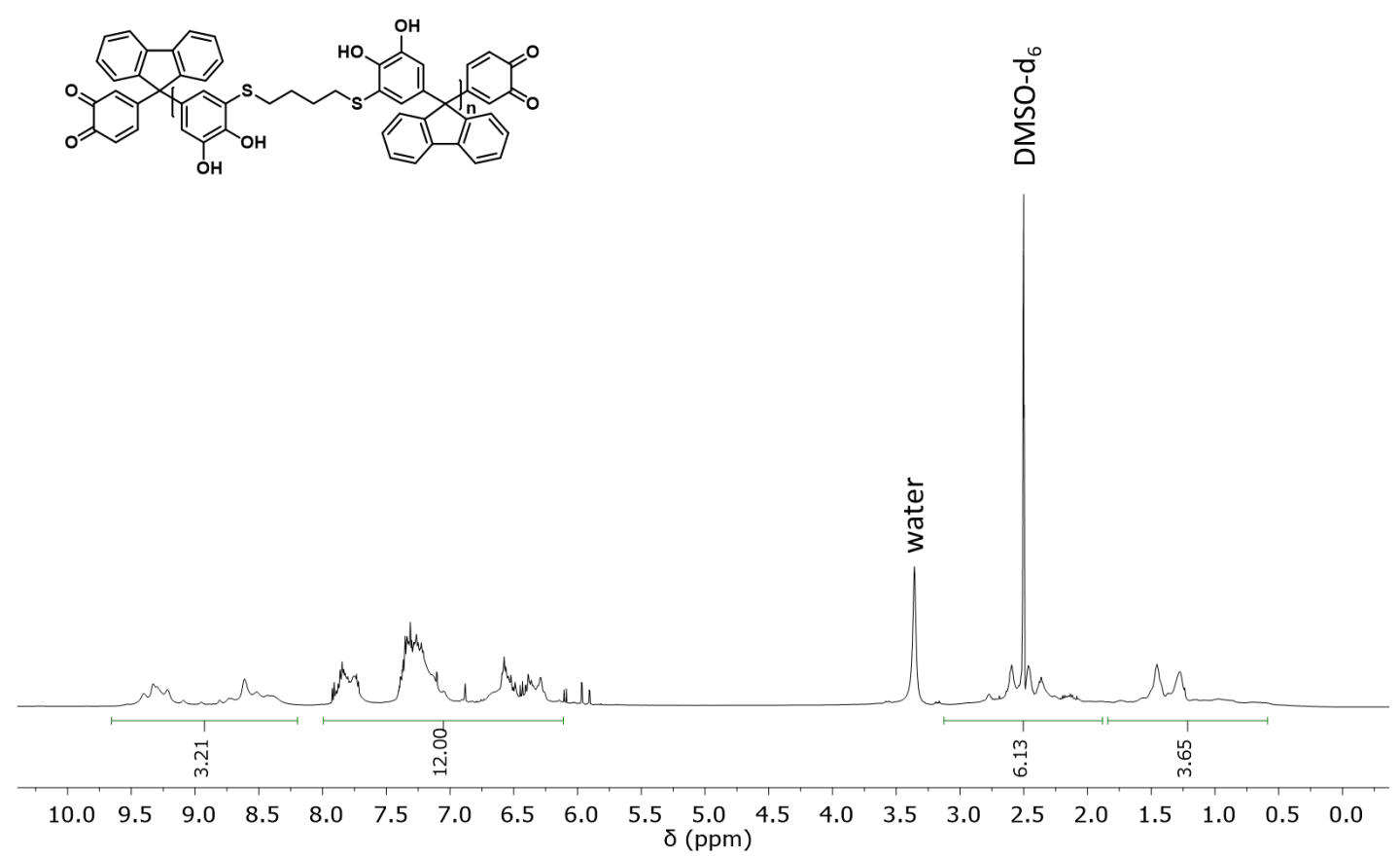

Figure S 103. ${ }^{1} \mathrm{H}-\mathrm{NMR}$-spectra of $\mathrm{p}$ (DCFL-BDT) in DMSO-d ${ }_{6}$.

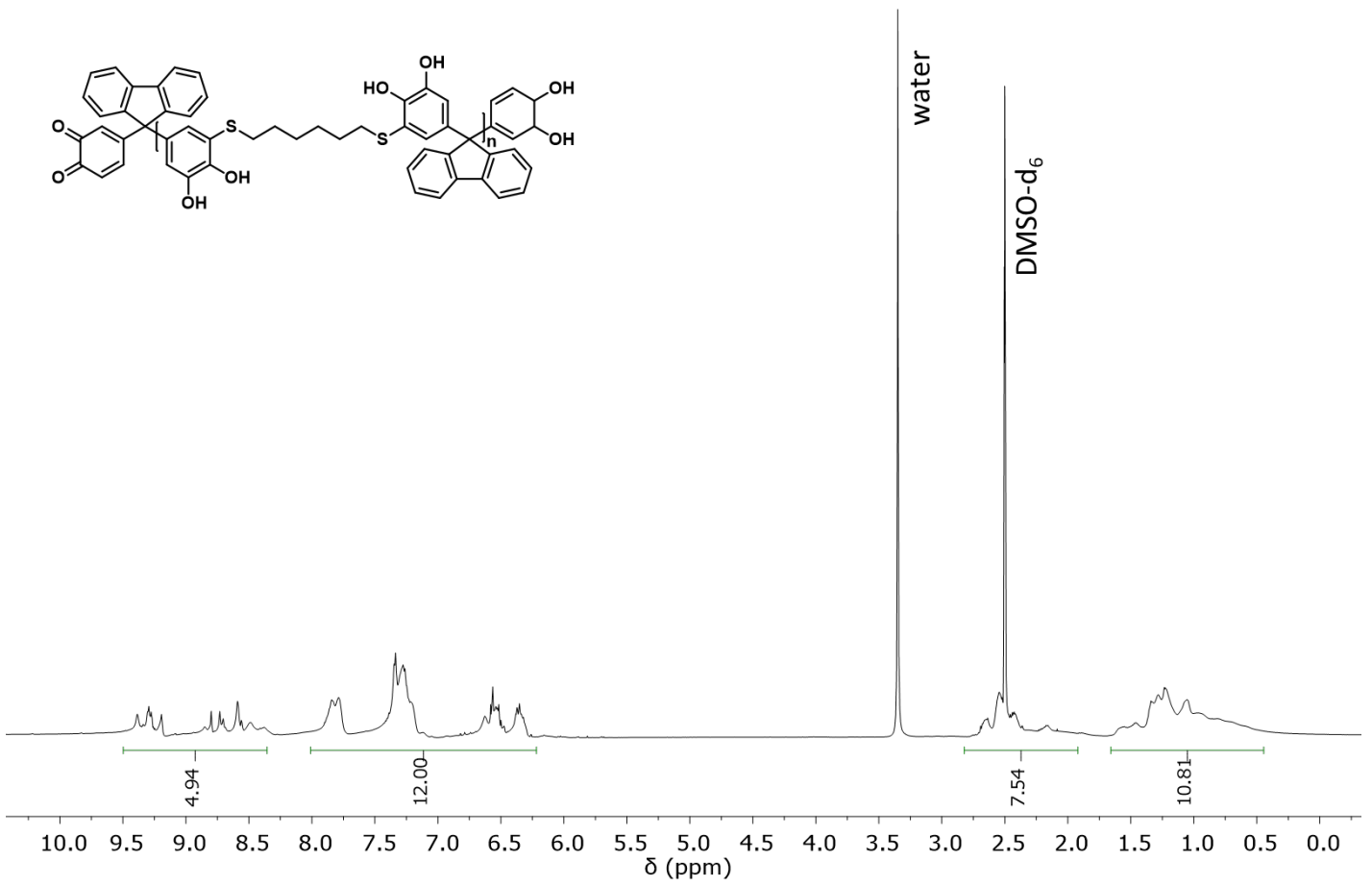

Figure S 104. ${ }^{1} \mathrm{H}-\mathrm{NMR}$-spectra of $\mathrm{p}$ (DCFL-HDT) in DMSO-d 6 . 


\section{References S.I.}

1. Frigerio, M.; Santagostino, M.; Sputore, S., A User-Friendly Entry to 2-Iodoxybenzoic Acid (IBX). J. Org. Chem. 1999, 64 (12), 4537-4538.

2. Plumb, J. B.; Harper, D. J., 2-Iodoxybenzoic acid. Chem. Eng. News 1990, 68 (29), 3.

3. Pei, J.; Hsu, C.-C.; Wang, Y.; Yu, K., Corona discharge-induced reduction of quinones in negative electrospray ionization mass spectrometry. RSC Advances 2017, 7 (69), 43540-43545. 